\title{
Bestimmung von Platzbesetzung und Bindungsenergien mittels Atomsondentomographie
}

\author{
Dissertation \\ zur Erlangung des Doktorgrades \\ der Mathematisch-Naturwissenschaftlichen Fakultäten \\ der Georg-August-Universität zu Göttingen
}

vorgelegt von
Torben Boll
aus Göttingen

Göttingen 2010 
D 7

Referent: Prof. Dr. Reiner Kirchheim Korreferent: Prof. Dr. Markus Münzenberg Tag der mündlichen Prüfung: 07. Mai 2010 



\section{Inhaltsverzeichnis}

1 Einleitung 3

$\begin{array}{lll}2 & \text { Grundlagen } & 7\end{array}$

2.1 Notation mit Millerschen Indizes . . . . . . . . . . . . 7

2.2 Intermetallische Phasen . . . . . . . . . . . . . . . 8

2.3 Titan-Aluminium-Legierungen . . . . . . . . . . . 8

2.4 Die $L 1_{0}$-Einheitszelle . . . . . . . . . . . . . . . . . . . . 10

2.5 Phasendiagramm von $\mathrm{CuAu} \ldots \ldots \ldots \ldots . \ldots . \ldots . \ldots 11$

2.6 Die $L 1_{2}$-Einheitszelle . . . . . . . . . . . . . . . . . . . . 11

3 Experimentelle Methoden $\quad 13$

3.1 Die Tomographische Atomsonde (TAP) . . . . . . . . . . . 13

3.1.1 Das generelle Funktionsprinzip . . . . . . . . . . . 13

3.1.2 Die Laserassistierte Atomsonde . . . . . . . . . . . . . . . 14

3.1.3 Der Rekonstruktionsalgorithmus . . . . . . . . . 15

3.2 Das Feldionenmikroskop $(\mathrm{FIM}) \ldots \ldots \ldots \ldots$

3.3 Das Transmissionselektronenemikroskop $($ TEM) . . . . . . . . . . 18

3.4 Focused Ion Beam $($ FIB $) \ldots \ldots \ldots \ldots . \ldots . \ldots . \ldots$ 
4.1 Bisherige Methoden zur Analyse der Kristallstruktur . . . . . . . . . 21

4.2 AtomVicinity . . . . . . . . . . . . . . . 22

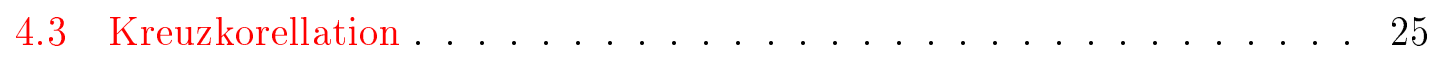

5 Probenpräparation $\quad 29$

5.1 Präparation für FIM und TAP . . . . . . . . . . . . 30

5.2 Präparation für TEM . . . . . . . . . . . . . . 31

5.3 Präparation für X-Ray . . . . . . . . . . . . . . 31

$6 \quad$ Experimentelle Ergebnisse $\quad 33$

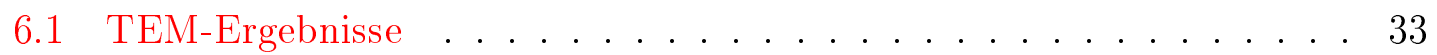

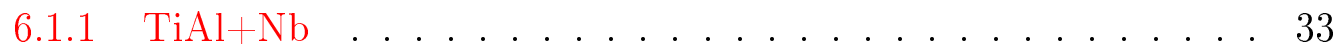

$6.1 .2 \operatorname{TiAlAg} \ldots \ldots \ldots \ldots \ldots \ldots . \ldots \ldots$

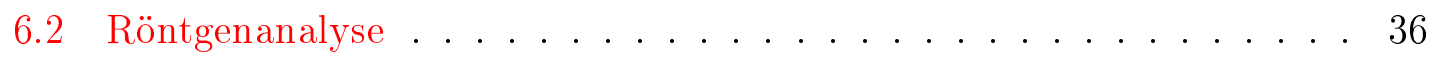

$6.2 .1 \mathrm{TiAl}+\mathrm{Cr} \ldots \ldots \ldots \ldots$

$6.2 .2 \mathrm{Cu}_{3} \mathrm{Au} \ldots \ldots \ldots \ldots \ldots \ldots \ldots$

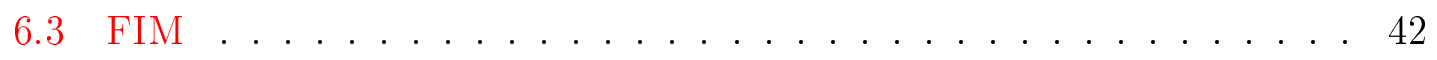

$6.3 .1 \mathrm{TiAl} \ldots \ldots \ldots \ldots \ldots . \ldots \ldots 4 . \ldots \ldots$

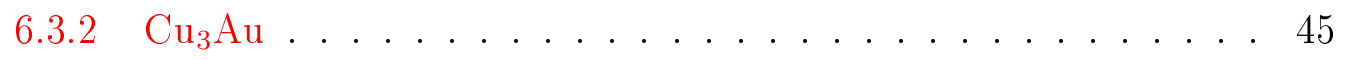

6.4 TAP: Feldverdampfungsfeldstärken . . . . . . . . . . . 46

6.4.1 Messparameter ..................... 46

6.4.2 Einstellen der Parameter der Rekonstruktion . . . . . . . . . 48

6.4.3 Massenspektren .................... 48

6.4.4 Bestimmung der Kristallstruktur . . . . . . . . . . 50 
6.4.5 Feldverdampfungsfeldstärken in $\mathrm{Ti}_{51} \mathrm{Al}_{48} \mathrm{Nb}_{1} \ldots \ldots . . .58$

6.4.6 Einfluss von Laserenergie und Temperatur . . . . . . . . . 61

6.5 TAP: Mikrostruktur . . . . . . . . . . . . . . 64

6.6 Lokaler Vergrößerungseffekt . . . . . . . . . . . . . . . 67

6.7 Verschmierung der Ebenen außerhalb der Pole . . . . . . . . 68

6.8 TAP: Feldverdampfungsfeldstärken . . . . . . . . . . . . 69

6.8.1 Abschätzung der Feldverdampfungsfeldstärken in TiAl $+\mathrm{X}$. . 70

6.8.2 $\mathrm{Cu}_{3} \mathrm{Au} \ldots \ldots \ldots \ldots \ldots \ldots \ldots 74 \ldots \ldots \ldots \ldots \ldots$

$6.9 \mathrm{Cu}_{3} \mathrm{Au}$ in Überstrukturrichtungen . . . . . . . . . . . . . 75

6.10 Bestimmung der Platzbesetzung in $\mathrm{TiAl}+\mathrm{X} \ldots \ldots . . . . . .76$

6.10.1 Platzbesetzung von $\mathrm{Nb} \ldots \ldots . \ldots 77$

6.10 .2 Platzbesetzung von $\mathrm{Ag} \ldots \ldots . \ldots 79$

6.10 .3 Platzbesetzung nach Isotopen getrennt . . . . . . . . . 81

6.10 .4 Platzbesetzung von $\mathrm{Cr} \ldots . . . . . . . . . .881$

$\begin{array}{lll}7 & \text { Simulationen } & 85\end{array}$

7.1 Berechnung von Bindungsenergien . . . . . . . . . . . 86

7.2 Modellierung der interatomaren Wechselwirkungen . . . . . . . 86

7.2.1 Grundlagen der DFT . . . . . . . . . . . . . 88

7.2.2 Gitterparameter durch DFT . . . . . . . . . . 89

7.2.3 DFT-Bindungsenergien für TiAl durch Leerstellen . . . . . . . 90

7.2.4 DFT-Bindungsenergien für TiAl durch Substitution . . . . . . 92

7.2.5 DFT-Bindungsenergien für TiAl $+\mathrm{X}$ durch Substitution . . . . 95

7.2.6 DFT-Bindungsenergien für $\mathrm{CuAu} \ldots . . \ldots 96$

7.3 Vereinfachtes Modell einer Atomsonde . . . . . . . . . . . . . 97 
7.3.1 Der Müller-Schottky Mechanismus . . . . . . . . . . . 98

7.3.2 Das verwendete Feldverdampfungsmodell . . . . . . . . . . 99

7.3.3 Ablauf der Simulation . . . . . . . . . . . . . . 101

7.4 Bestimmung von $D$ mit Wolfram . . . . . . . . . . . . . . 103

7.5 Simulationsergebnisse für $\mathrm{Cu}_{3} \mathrm{Au} \ldots \ldots . \ldots . \ldots . \ldots 707$

7.5.1 Simulationsparameter . . . . . . . . . . 107

7.5.2 Bestimmung der $\mathrm{Cu}$-Au-Bindungsenergie . . . . . . . . . 109

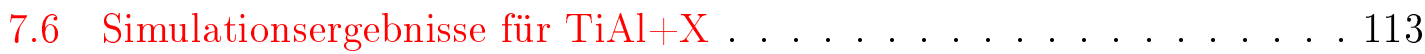

7.6.1 Simulationsparameter . . . . . . . . . . . 113

7.6.2 TiAl-Bindungspotentiale . . . . . . . . . . . 114

7.6.3 Abschätzung der Ti-Al-Bindungsenergie . . . . . . . . . 117

7.6.4 Auflösbarkeit der Netzebenen . . . . . . . . . . . . . 118

7.6.5 Die nichtgeordnete $[100]$-Richtung . . . . . . . . . . . 118

7.6.6 Die Überstrukturrichtungen . . . . . . . . . . . . 119

7.6.7 Einfluss des Ordnungsgrades . . . . . . . . . . . . 121

7.7 Ausblick ............................ 122

8 Zusammenfassung 125

Literaturverzeichnis

A Quellcodeauszüge SIMTAP 135

B Al8at.\%Li: Ein nicht ganz so einfaches System

Danksagung 


\section{Kapitel 1}

\section{Einleitung}

Die Platzbesetzung ternärer Elemente in einer intermetallischen Phase ist ein wichtiges Betätigungsfeld der Materialphysik. Bisher sind zur Untersuchung dieser Phänomene röntgenbasierte Methoden sowie Atom Location by Channeling Enhanced Microanalysis (ALCHEMI) [Jone2003], ein auf Transmissionselektronenmikroskopie aufbauendes Verfahren, üblich. In dieser Arbeit liegt der Fokus auf der Nutzung der Atomsondentomographie. Diese hat hat gegenüber ALCHEMI den Vorteil, dass auch leichte Elemente wie Lithium nachgewiesen werden können. Zudem ist es möglich, verschiedene Isotope desselben Elements unabhängig voneinander zu betrachten. Nicht zuletzt kann eine Ortsabhängigkeit der Parameter innerhalb des analysierten Volumens mit um eine Größenordnung besserer räumlicher Auflösung untersucht werden. Unter optimalen Bedingungen kann ein Volumen von wenigen $\mathrm{nm}^{3}$ ausreichen, um mit der in dieser Arbeit vorgestellten Methode die Platzbesetzung zu bestimmen.

Es gab bereits in der Vergangenheit Ansätze, die Platzbesetzung anhand von Atomsondendaten zu bestimmen. So betrachteten Kim et al. [Kim1997] Konzentrationsprofile in $\mathrm{L} 1_{0}$-Titan-Aluminium entlang von Überstrukturrichtungen und waren in der Lage Peaks zu identifizieren, die sie als eine Ebenenabfolge interpretierten. Anhand des Konzentrationsverlaufs des hinzulegierten Ruthenium konnten sie eine bevorzugte Platzbesetzung angeben. Diese direkte Methode ist allerdings nicht immer möglich, da eine Unterscheidung von Ebenen aufgrund von durch die Feldverdampfung produzierten Artefakten häufig nicht durchgeführt werden kann (Kap.4). Durch statistische Methoden lässt sich allerdings dennoch eine Unterscheidung verschiedener Ebenen erreichen [Boll2007]. 
Um zu überprüfen, ob die Interpretation der experimentellen Daten im Hinblick auf die Platzbesetzung korrekt ist, ist es nötig, Simulationen durchzuführen. Vurpillot et al. [Vurp2004a] simulierten Feldverdampfungssequenzen einer intermetallischen Phase. Dabei wurde allerdings für jedes Element eine Feldverdampfungsfeldstärke vorgegeben, die unabhängig von den lokalen Nachbarschaftsverhältnissen an der Oberfläche ist. Die Berechnung der Feldverdampfungsfeldstärken erfolgt nach dem Modell von Müller und Schottky [Muel1969] über die Ionisationsenergie, Austrittsarbeit und Sublimationsenergie. In dieser Arbeit wird die Sublimationsenergie durch einen Term, der die lokalen Bindungen berücksichtigt, ersetzt. Die dadurch möglichen Simulationen erlauben eine weit genauere Vorhersage der Effekte, die durch einen geringeren Ordnungsgrad oder die Hinzulegierung ternärer Elemente bewirkt werden. Zudem wird ein quantitativer Vergleich der Ergebnisse für unterschiedliche kristallographische Richtungen möglich, was, im Vergleich mit experimentellen Ergebnissen, eine Abschätzung von A-B-Bindungsenergien erlaubt (Kap. 7.5.2).

Der Ordnungsgrad einer intermetallischen Phase, aber auch die Platzbesetzung hinzulegierter Elemente sind für mechanische und chemische Eigenschaften von großer Bedeutung. Als Modellsystem wurden Titan-Aluminium-Legierungen untersucht, die von großem Interesse für Hochtemperaturanwendungen sind [Gilc2001], bei denen ein geringes Gewicht von Bedeutung ist. In dieser Arbeit wurde vorwiegend die $\gamma$-L1 $1_{0}$-Phase untersucht, welche Überstrukturrichtungen mit einer Abfolge reiner Ti- und Al-Ebenen aufweist. Der Einfluss auf die Feldverdampfung sowie die Platzbesetzung von hinzulegiertem Nb, Ag und Cr wurden untersucht. Als weiteres Modellsystem wurde $\mathrm{Cu}_{3}$ - $\mathrm{Au}$ mit seiner $\mathrm{L}_{2}$-Phase herangezogen. Hier liegen in den Überstrukturrichtungen Abfolgen reiner $\mathrm{Cu}$-Ebenen und gemischter $\mathrm{Cu} / \mathrm{Au}$-Ebenen vor.

An dieser Stelle ist es sinnvoll kurz auf die Gliederung dieser Arbeit einzugehen. Nach den, für Fachkundige weniger interessanten, Grundlagen (Kap. 2, 3) werden in Kap. 4 die entwickelten Analysealgorithmen vorgestellt. Nach der Präparation in Kap. 5 wird in den Kapiteln 6.1 bis 6.2 vor allem eine allgemeinene Charakterisierung des Materials durchgeführt. Ein wichtiger Teil der Arbeit, die Betrachtung der Feldverdampfungsfeldstärken, die sowohl für verschiedene Richtungen als auch für verschiedene Elemente (sogar innerhalb derselben Legierung) unterschiedlich sind, wird in den Kapiteln 6.3 bis 6.8 behandelt. Dabei ist es erstmals gelungen, quantitative Aussagen über die Unterschiede der Feldverdampfungsfeldstärken für verschiedene Richtungen zu machen. 
Die Bestimmung der Platzbesetzung anhand von Atomsondendaten wird in Kap. 6.10 beschrieben. Diese Arbeit legt in diesem Bereich Grundlagen, indem eine neue Methode vorgestellt wird, die eine quantitative Bestimmung der Platzbesetzung erlaubt.

Im abschließenden Kapitel 7 wird eine Möglichkeit zur Simulation von Atomsondendaten vorgestellt und mit experimentellen Ergebnissen aus Kap. 6 verglichen. Dazu werden Berechnungen mit der Dichtefunktionaltheorie durchgeführt, aus denen Bindungsenergien für die Simulation von $\mathrm{TiAl}+\mathrm{X}$ und $\mathrm{Cu}_{3} \mathrm{Au}$ hergeleitet werden. Dies ist allerdings, wie in Kap. 7.5.2 für $\mathrm{Cu}_{3} \mathrm{Au}$ demonstriert wird, nicht grundsätzlich erforderlich. Allein durch den Vergleich von Simulation und Experiment lässt sich die Stärke der Cu-Au-Bindung angeben. Für TiAl mit Zusätzen konnte durch Simulationen die Verdampfungsreihenfolge in geordnete und nichtgeordnete Richtungen erklärt werden, was für die Platzbestimmung von großer Bedeutung ist. 
KAPITEL 1. EINLEITUNG 


\section{Kapitel 2}

\section{Grundlagen}

\subsection{Notation mit Millerschen Indizes}

Üblicherweise werden zur Bezeichnung von Richtungen und Ebenen in einem Kristallgitter Millersche Indizes [Kitt1969] verwendet. Dies geschieht auch in dieser Arbeit. Wichtig ist, die Bedeutung der unterschiedlichen Klammern zu erkennen. Eckige [--Klammern bezeichnen eine bestimmte kristallographische Richtung. Spitz zulaufende $<>$ - Klammern umfassen alle gleichartigen Richtungen. Mit $<100>$ sind also [100], [010], [001], [100], [010] sowie [001] gemeint. Entsprechendes gilt bei der Bezeichnung von Ebenen wie etwa bei den Polen in Feldionenmikroskopie-Aufnahmen. Hier bezeichnen normale ()-Klammern einen Pol und geschweifte \{\} -Klammern alle gleichartigen Pole.

Es ist eine Besonderheit zu beachten. Üblicherweise wäre, im Falle einer auf einem kubischflächenzetrierten (kfz) Gitter beruhenden Struktur, die Auslöschungsregel zu beachten, dass bei einer Beugungsanalyse nur entweder gerade oder ungerade Indizes auftauchen. Da wir geordnete Legierungen betrachten, können jedoch diese Regeln gebrochen werden. Interessant ist hierbei, dass die Bezeichnung einer geordneten Ebenenabfolge etwa bei der L1 $1_{0}$-Struktur (Abb. 2.3) als (001) durchaus auch bei der Bezeichnung von Ebenen in einer Atomsondenmessung richtig ist. Obwohl natürlich im Grunde die Ebenen im Abstand von (002)-Ebenen aufeinanderfolgen, werden von der Atomsonde oft nur Abstände wie für (001)-Ebenen detektiert. Dies wird in späteren Kapiteln (Kap. 4) ausführlicher diskutiert. Im Text ist meistens die kristallographische Richtung mit den kleinstmöglichen ganzzahligen Komponenten 
angegeben, da dadurch eventuellen Verwirrungen bzgl. Ebenenabständen vorgebeugt wird $^{1}$.

Um den Leser nicht durch die Angabe zu vieler Richtungen unnötig zu verwirren, werden in dieser Arbeit Richtungen, die gleichartig sind, durch einen Stellvertreter bezeichnet. Dieser umfasst nicht nur, wie es auch sonst üblich ist, die entgegengesetzte Richtung sondern alle Richtungen, die die gleiche Ebenenabfolge aufweisen. Im Falle der $\mathrm{L1}_{0}$-Struktur bezeichnet [100] somit auch gleichzeitig die [010]-Richtung, wohingegen [001] nur für sich selbst und die entgegengesetzte Richtung steht.

\subsection{Intermetallische Phasen}

Eine geordnete Phase liegt dann vor, wenn man die Positionen der Elemente in einer Legierung jeweils durch eigene Untergitter beschreiben kann. Es gibt verschiedene Gründe, warum sich intermetallische Phasen ausbilden können. Zum einen kann allein die räumliche Ausdehnung (Laves-Phasen,Hägg-Phasen) eine Rolle spielen oder die Valenzelektronenkonzentration (Hume-Rothery-Phasen). Für diese Arbeit sind Substitutionsmischkristalle von Interesse, die durch günstige Verhältnisse der Vertauschungsenergien entstehen. Selbstverständlich muss zur Ausbildung einer Ordnung selbige energetisch gegenüber dem entordneten Zustand begünstigt sein. Damit eine intermetallische Phase in einer A-B-Legierung vorliegen kann, muss für die Bindungsenergien $\Lambda$ in der entsprechenden Kristallstruktur gelten [Gott2001, Haas1994]:

$$
\Lambda_{A-B}-\frac{\Lambda_{A-A}+\Lambda_{B-B}}{2}>0
$$

Diese Gleichung beschreibt üblicherweise die Paarvertauschungsenergien, die meist mit $\epsilon$ bezeichnet werden. Das Vergleichszeichen zeigt hier zudem in die andere Richtung, da hohe Bindungsenergien eine bessere Bindung bedeuten sollen. Allerdings ist es für spätere Kapitel sinnvoll, bereits hier die Bindungsenergien einzuführen.

\subsection{Titan-Aluminium-Legierungen}

Titan-Aluminium-Basislegierungen sind als Hochtemperatur-Materialien für viele Anwendungen interessant. Vor allem aufgrund des geringen Gewichts ist es sinnvoll

\footnotetext{
${ }^{1}$ Die [100]-Richtung ist z.B. die gleiche Richtung wie die [200]-Richtung, lediglich die Länge des Vektors unterscheidet sich.
} 


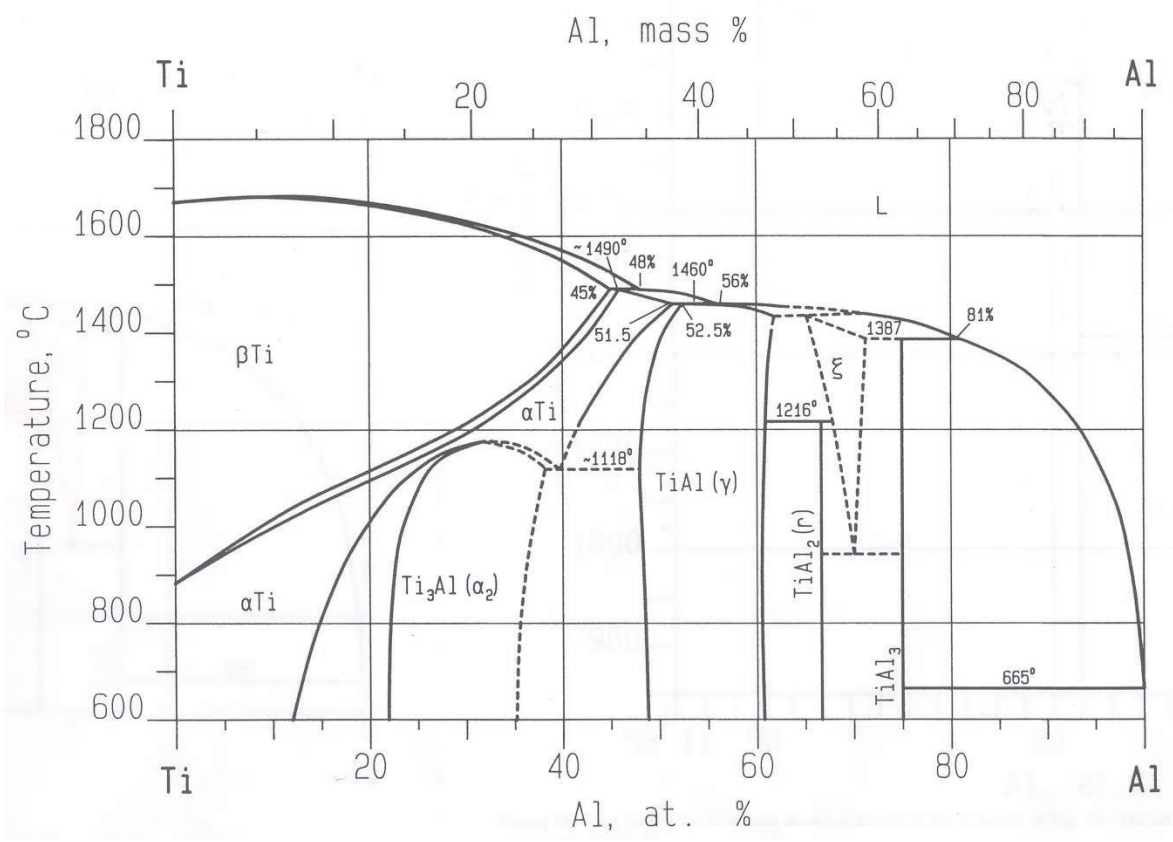

Abbildung 2.1: Phasendiagramm von TiAl. Man beachte den Existenzbereich der für diese Arbeit interessanten $\gamma$-Phase. Entnommen aus [Petz1993].

eine Anwendung etwa in Flugzeugturbinen anzudenken [Gilc2001]. Die mechanischen Eigenschaften einer Legierung mit $\gamma / \alpha_{2}$-Lamellenstruktur [Cald2002] sind bei hohen Temperaturen sehr geeignet. Bei niedrigen Temperaturen sinkt die Duktilität jedoch stark ab. Durch Zugabe von Elementen wie Chrom, Niob, Wolfram, Vanadium und anderen versucht man dies zu verbessern. Diese Zusätze beeinflussen die Fähigkeit der Versetzungen zu Wandern und ändern dadurch die Duktilität. Durch Niob wird einerseits die Härte erhöht, andererseits aber auch die Oxidationsresistenz verbessert, weswegen nahezu alle bisher entwickelten TiAl-Legierungen Niob enthalten. In Abb. 2.1 ist das Phasendiagramm von Titan-Aluminium zu sehen. Von Interesse für diese Arbeit sind die $\alpha_{2}$-Phase, die eine $\mathrm{D} 0_{19}$-Struktur besitzt, und die $\gamma$-Phase mit L1 $1_{0}$-Struktur. Die hergestellten Proben enthalten überwiegend diese beiden Phasen. In Abb. 2.2 ist das ternäre Phasendiagramm von Ti-Al-Nb zu sehen. Für die verwendeten Niob-haltigen Legierungen $\mathrm{Ti}_{51} \mathrm{Al}_{48} \mathrm{Nb}_{1}, \mathrm{Ti}_{47} \mathrm{Al}_{48} \mathrm{Nb}_{5}$ und $\mathrm{Ti}_{42} \mathrm{Al}_{48} \mathrm{Nb}_{10}$ erwartet man einen großen Anteil der $\gamma$-Phase. Leider standen für die Ti-Al-Legierungen mit Silber und Chrom keine ternären Phasendiagramme zur Verfügung. Da der Anteil der Zusätze jedoch gering ist, erwartet man auch bei $\mathrm{Ti}_{46} \mathrm{Al}_{52} \mathrm{Ag}_{2}$ und $\mathrm{Ti}_{52} \mathrm{Al}_{45} \mathrm{Cr}_{3}$ ebenfalls hohe Anteile der $\gamma$-Phase. 


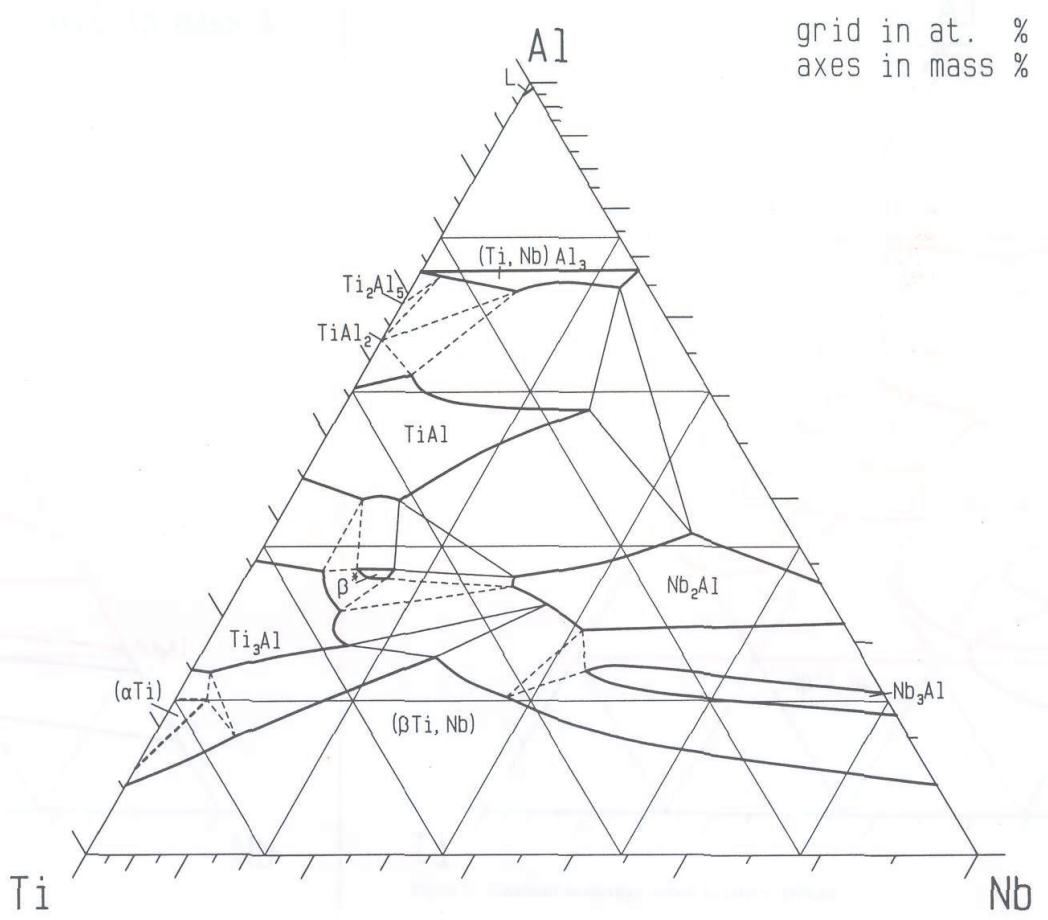

Abbildung 2.2: Ternäres Phasendiagramm von TiAlNb bei der verwendeten Auslagerungstemperatur von 1273 K. Entnommen aus [Petz1993].

\subsection{Die $L 1_{0}$-Einheitszelle}

Die $\gamma$-Phase von TiAl besitzt die $\mathrm{L1}_{0}$-Struktur, die in Abb. 2.3 zu sehen ist. In [001]-Richtung findet man abwechselnd Ti- und Al-Ebenen. Diese Richtung wird als geordnete Richtung oder als Überstrukturrichtung bezeichnet. In [010]- und [100]Richtung trifft man auf gemischte Ebenen mit jeweils gleichem Anteil an Ti und Al. Alle $<111>$-Richtungen sind ebenfalls gemischt besetzt. Interessant für die späteren Kapitel ist, dass es noch eine weitere niedrigindizierte Überstrukturrichtung, nämlich [110], gibt. Die [011]- sowie die [011]-Richtung sind nicht geordnet. Die nächsthöherindizierte Überstrukturrichtungen sind die [201]- und die [211]-Richtungen.

Die Einheitszelle ist zudem gestaucht. Nach [Meno1996, Zamb2009] beträgt $c / a=1.02$, was auch mit den für diese Arbeit durchgeführten Röntgenmessungen in Kap. 6.2.1 übereinstimmt. Dabei bezeichnet $c$ die [001]-Kantenlänge und $b=a$ die beiden anderen Kantenlängen der Einheitszelle. Nach [Meno1996] sind $c=0.40786 \mathrm{~nm}$ und $a=b=0.39847 \mathrm{~nm}$. In dieser Arbeit wurde mit der Dichtefunktionaltheorie (DFT) ebenfalls ein $c / a=1.02$ errechnet mit $a=b=0.3989 \mathrm{~nm}$ und $c=0.4057 \mathrm{~nm}$ (Kapitel 7.2.3). Die Dehnung ist also recht klein. 


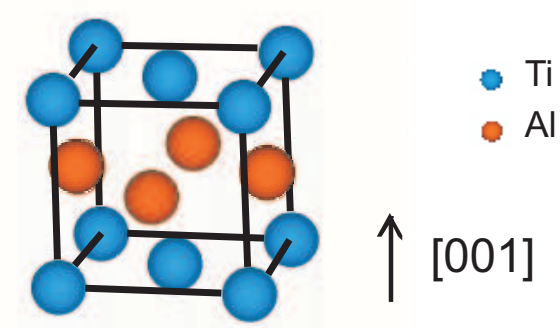

Abbildung 2.3: Die L10-Einheitszelle von TiAl. In [001]-Richtung findet man abwechselnd Ti- und Al-Ebenen.

\subsection{Phasendiagramm von $\mathrm{CuAu}$}

In Abb. 2.4 ist das Phasendiagramm von $\mathrm{CuAu}$ zu sehen. Es gibt im Wesentlichen 3 geordnete Phasen, wobei in dieser Arbeit die $\mathrm{Cu}_{3} \mathrm{Au}$-Phase untersucht wurde. Die mit der römischen Ziffer II bezeichneten Phasen basieren auf der gleichen Kristallstruktur, wie die mit I bezeichneten Phasen, enthalten aber alle paar Atomlagen (im Fall von CuAu alle 5) eine Zwillingsgrenze [Haas1994]. Für die in dieser Arbeit verwendeten $\mathrm{Cu}_{3} \mathrm{Au}$-Proben ist somit die $\mathrm{L}_{2}$-Phase $\mathrm{zu}$ erwarten.

\subsection{Die $L 1_{2}$-Einheitszelle}

$\mathrm{Cu}_{3} \mathrm{Au}$ besitzt eine $\mathrm{L}_{2}$-Einheitszelle, wie in Abb. 2.5 dargestellt. Der Unterschied zur $\mathrm{L} 10_{0}$-Einheitszelle in Abb. 2.3 besteht darin, dass die Atome der oberen und der unteren Seitenfläche vom gleichen Typ wie die Atome auf der Zwischenebene sind. Zudem gibt es keine Stauchung der Einheitszelle in eine der Richtungen. Der Gitterparameter ist laut [Haas1994] $0.374 \mathrm{~nm}$. Durch die in dieser Arbeit verwendete DFT errechnet sich ein Gitterparameter von $0.375 \mathrm{~nm}$. Die am niedrigsten indizierten Überstrukturrichtungen sind die $<100>$-Richtungen sowie die $<110>$ Richtungen. Hier wechselt sich eine gemischt 50/50-besetzte Ebene mit einer reinen Cu-Ebene ab. Die $<111>$-Richtungen sind die am niedrigst indizierten nicht geordneten Richtungen. 


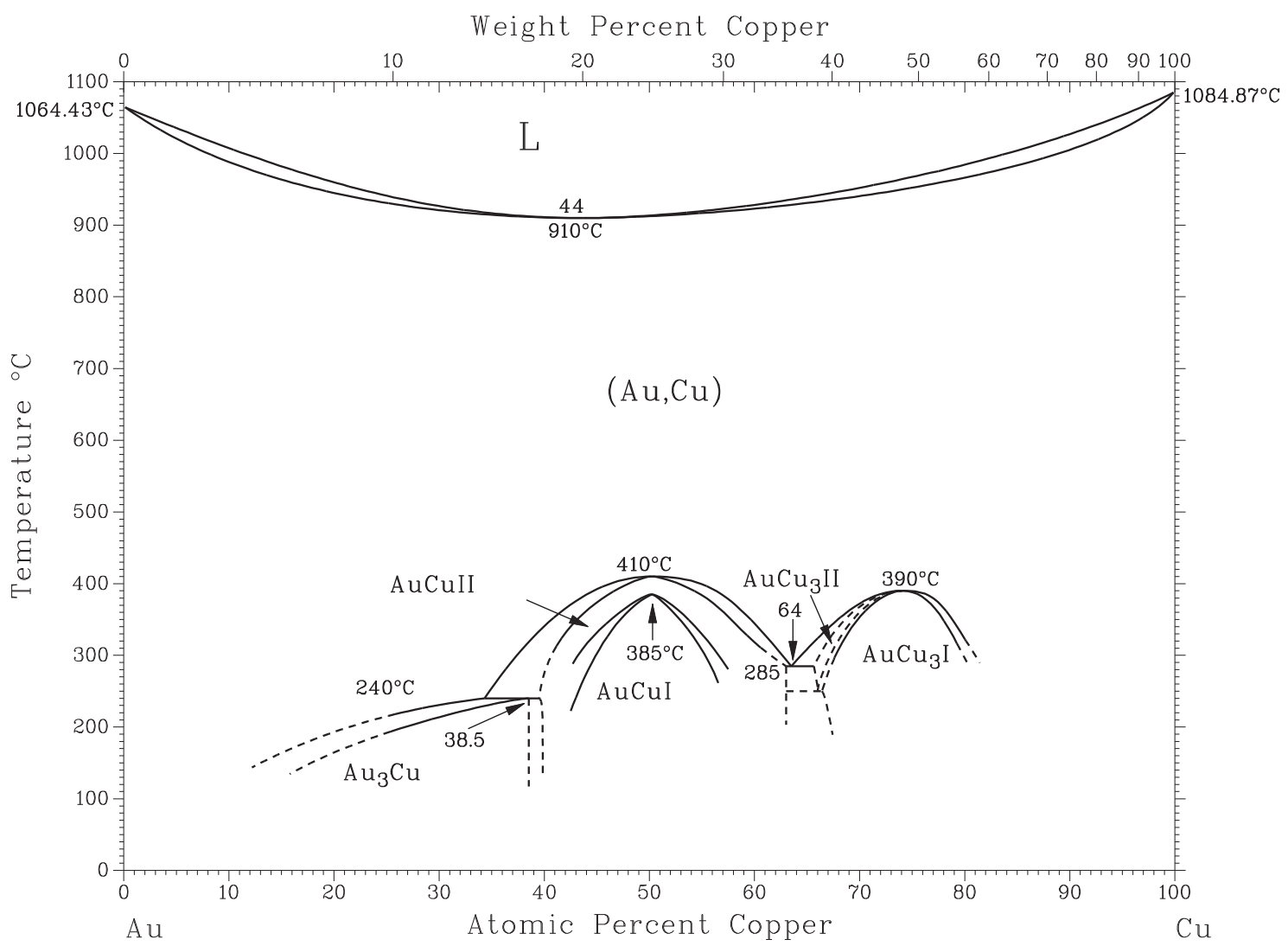

Abbildung 2.4: Das Phasendiagramm von CuAu [Mass1990]. Für diese Arbeit ist die $\mathrm{Cu}_{3} \mathrm{Au}$-Phase von Interesse.

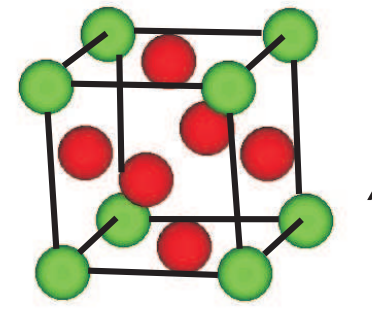

(a) Einheitszelle

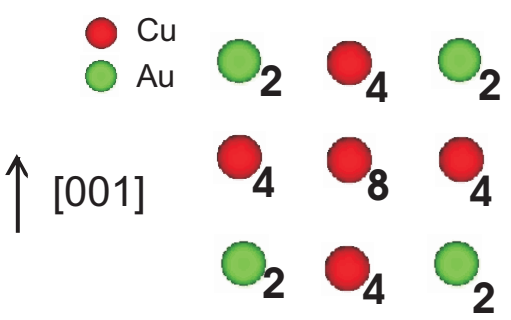

(b) Projektion auf (c) Projektion gedreht $(x, z)$-Ebene

Abbildung 2.5: (a): Die $\mathrm{L}_{2}$-Einheitszelle von Cu 3 Au. Auf den Ecken befinden sich AuAtome, auf den Seitenflächen Cu-Atome. Alle $<001>-$ Richtungen sind offensichtlich gleichartig. Die nächst höher indizierten Überstrukturrichtungen sind die $\langle 110\rangle$-Richtungen. In (b) ist die Projektion auf die $(x, z)$-Ebene dargestellt um die Ebenenabfolge zu verdeutlichen. Durch die Ziffern ist jew. die Anzahl der Atome pro Einheitszelle multipliziert mit 8 angegeben, die von der zugehörigen Kugel repräsentiert werden. In (c) ist die Projektion um $45^{\circ}$ gedreht. In z-Richtung befindet sich nun eine (220)-Ebenenabfolge. Die zusätzlichen blassen Atome zeigen, wie sich die Projektion für breitere und höhere Zellen fortsetzt. 


\section{Kapitel 3}

\section{Experimentelle Methoden}

\subsection{Die Tomographische Atomsonde (TAP)}

Die tomographische Atomsonde (engl. Tomgraphic Atom Probe - TAP ${ }^{1}$ ) ist ein Analysegerät, das eine chemische Untersuchung der Mikrostruktur bis in den Subnanometerbereich erlaubt.

\subsubsection{Das generelle Funktionsprinzip}

In Abb. 3.1 ist der Aufbau des TAP skizziert. An einer sehr feinen Spitze mit einem Radius $R$ von 10 bis $100 \mathrm{~nm}$ wird eine Basispannung $U_{\text {Basis }}=2 . .20 \mathrm{kV}$ angelegt, die gerade so hoch ist, dass sie gerade noch nicht ausreicht um Atome von der Spitze abzulösen. Zusätzlich wird eine Pulsspannung $U_{P u l s}$ angelegt, die dafür sorgt, dass einzelne Atome feldverdampfen. Diese treffen auf die Multikanalplatten, auf welchen sie Elektronenkaskaden auslösen, die auf dem dahinter liegenden Detektor gemessen werden. Im Falle der Göttinger Atomsonde ist dieser Detektor eine Multianodenanordnung. Die $(x, y)$-Position des Atoms wird aus der Ermittlung des Ladungsschwerpunktes bestimmt. Dafür ist es natürlich nötig, dass eine sinnvolle Anzahl von Anoden ein Signal liefert. Am besten ist es, wenn 3 bis 5 von ihnen angesprochen werden. Die Anzahl kann man durch eine Veränderung der an den Multikanalplatten anliegenden Spannung optimieren. Die optimalen Parameter sind dabei vor allem materialabhängig. Eine andere Detektorart ist der Delay Line Detector (DLD). Bei diesem befindet sich hinter den Multikanalplatten ein Netz aus drei

\footnotetext{
${ }^{1}$ Da die englische Abkürzung gebräuchlicher ist, wird sie anstelle von AS verwendet.
} 
verschieden orientierten Kupferdrähten. Die Position wird hier über die Laufzeitunterschiede, mit denen das eintreffende Signal an den 6 Drahtenden detektiert wird, bestimmt. Die $z$-Position der Atome wird anhand ihrer Auftreffreihenfolge ermittelt.

\section{Spitzenapex}

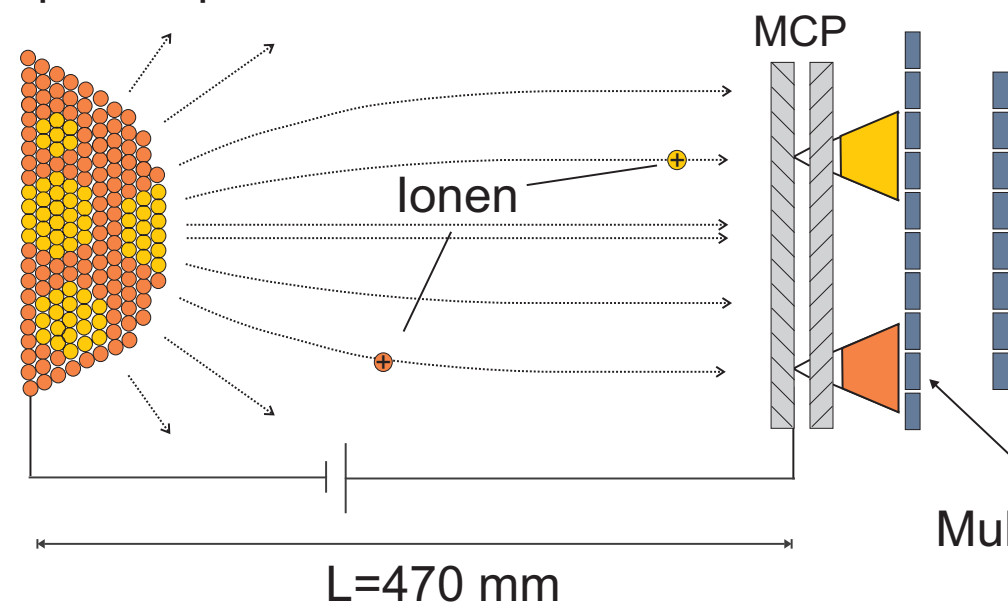

TAP- Detektor

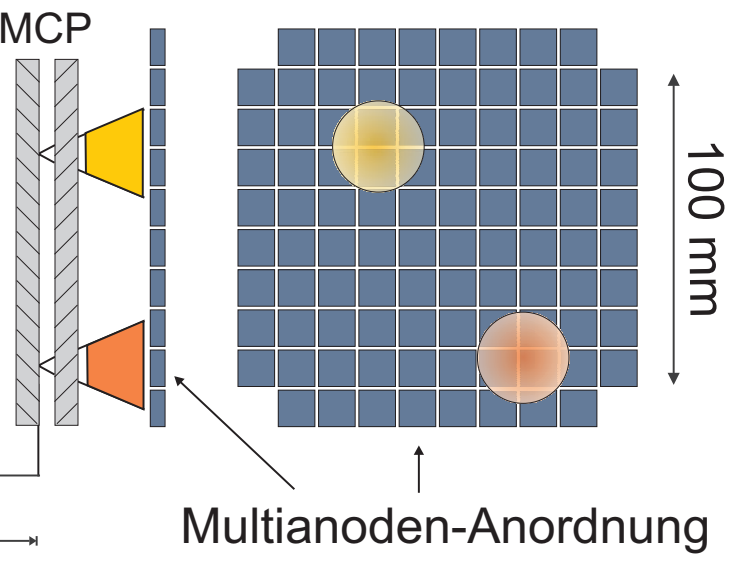

Abbildung 3.1: Die Göttinger Atomsonde: Von einer Spitze mit einem Spitzenradius von $100 \mathrm{~nm}$ oder weniger (links) werden bei einer hohen Spannung durch zusätzlich angelegte Spannungspulse einzelne Atome feldverdampft. Die Atome verursachen an den Multikanalplatten (MCP) Elektronenkaskaden, welche auf der Multinaodenanordnung detektiert werden. Der Aufbau befindet sich im Vakuum mit einem Restgasdruck $<10^{-9}$ mbar.

\subsubsection{Die Laserassistierte Atomsonde}

Für einen Teil der Versuche wurde die Laser Assisted Local Electrode Atom Probe (LEAP), das Imago LEAP ${ }^{\mathrm{TM}} 3000 \mathrm{X}$ Si des Fraunhofer-Instituts in Dresden, verwendet. Der verwendete Laser arbeitet bei einer Wellenlänge von $532 \mathrm{~nm}$ und einer Pulslänge von 10 ps mit einem Spotdurchmesser unter $10 \mu \mathrm{m}$ [Bunt2007]. Die Funktionsweise einer Atomsonde im Laser-Modus ist vergleichbar mit dem gewöhnlichen TAP. Allerdings wird anstelle der Pulsspannung ein Laserpuls zum Evaporieren der Atome von der Spitze verwendet. Die Ablösung findet dabei, wie zumindest für den von uns verwendeten Laser mit Pulsen im ps-Bereich wenig umstritten ist, thermisch statt. Für Laser, die mit Pulsen im Femtosekundenbereich arbeiten, gibt es Hinweise darauf, dass auch ein optischer feldbasierter Prozess stattfindet [Deco2008, Vurp2007].

Mit "Local Electrode" wird die Form und Position der Gegenelektrode bezeichnet. Diese besitzt die Form eines Hohlkegels mit abgeschnittenem Oberteil, welcher sehr dicht $(1 \mu \mathrm{m})$ an die untersuchte Spitze herangefahren wird. Durch diese Bauweise ist es möglich, eine große Zahl von Proben -etwa auf einem Siliziumwafer- vorzubereiten 
und nacheinander vor die Elektrode zu bewegen. Dies lässt in vielen Fällen eine schnellere und besser standardisierte Präparation zu.

Ein weiterer wichtiger Vorteil der Dresdener Sonde gegenüber der Göttinger Sonde ist die mögliche $(x, y)$-Ausdehnung einer Messung. Die Göttinger Atomsonde erlaubt ein Quadrat mit einer Diagonale, die einem Winkel von etwa $25^{\circ}$ entspricht, zu analysieren. Die Dresdener Sonde hingegen ermöglicht die Analyse eines kreisförmigen Bereichs mit einem Durchmesser, der einem Winkel von etwa $50^{\circ}$ entspricht. Dadurch ist es möglich, mehr als nur einen niedrig indizierten Pol im Analysevolumen zu haben.

\subsubsection{Der Rekonstruktionsalgorithmus}

Obwohl bei heutigen Atomsonden aufgrund des größeren Analysebereichs die benötigte Näherung, von einer Zentralprojektion auszugehen, nur noch eingeschränkt zutrifft, wird von aktuellen Programmen nach wie vor der Rekonstruktionsalgorithmus, wie von Bas et al. [Bas1995] beschrieben, verwendet.

Die Ermittlung der $(x, y)$-Koordinaten eines Atoms erfolgt hierbei durch Berechnung des Vergößerungsfaktors $\eta$. Dieser ist gegeben durch

$$
\eta=\frac{L}{(m+1) R \xi} \operatorname{mit} R=\frac{V}{E_{\text {Evap }} \beta} .
$$

Dabei sind $L$ der Abstand Spitze-Detektor, $R$ der Krümmungsradius am Apex, $E_{\text {Evap }}$ die Evaporationsfeldstärke, $V$ das Potential und $m$ ein Parameter, der von der Ausrichtung der Spitze abhängt. Aufgrund gekrümmter Ionentrajektorien ist $\eta$ um einen weiteren Faktor $(\xi \sim 1.6)$ verringert. Ist die Spitze nicht gegenüber der Längsachse verkippt, so ist $m=0$. Der Parameter $\beta$ hängt von der Spitzengeometrie, bei einer idealen Spitze also vor allem vom Schaftwinkel ab und liegt typischerweise im Intervall $[2,8]$ (nach [Bas1995]). Ist $(X, Y)$ die ermittelte Position auf der Detektorfläche, so folgt

$$
x=\frac{X}{\eta} \text { und } y=\frac{Y}{\eta} .
$$

Bei dieser Berechnung der $(x, y)$-Position, mit dem Ursprung $(0,0)$ in der Spitzenmitte, geht man von einer ortsunabhängigen Vergrößerung bei einer stabilen Spitzengeometrie aus. Dies ist in der Realität nur eingeschränkt zutreffend. Es kommt an einigen Stellen zu, später in Kapitel 6.6 beschriebenen, lokalen Vergrößerungseffekten an den aus der Spitze herausragenden Stellen. Zudem ist die Vergrößerung 
im Grunde winkelabhängig. Bei der Göttinger Sonde mit ihrem kleinen Analysevolumen spielt dies keine Rolle. Bei moderneren Sonden ist ein winkelabhängiger Kompressionsfaktor nötig, um dies zu korrigieren.

Die $z$-Koordinate eines Atoms wird aus der Detektionsreihenfolge bestimmt. Dabei geht man davon aus, dass Atome, die später auf dem Detektor eintreffen, tiefer im Material lagen. Zur Veranschaulichung ist es sinnvoll von einem Pol in der Mitte auszugehen, dessen Ebenen somit senkrecht zur Spitzenrichtung liegen Die zKomponente des $N$-ten Atoms berechnet sich somit unter der Annahme, dass alle Atome dasselbe Volumen $V_{a t}$ einnehmen, als:

$$
z_{0}=\sum_{i=1}^{N-1} K_{0} / V_{i}^{2} \text { mit } K_{0}=\frac{\left(L E_{E v a p} \beta\right)^{2}}{S_{D} Q(m+1)^{2}} V_{a t} .
$$

Hierbei sind $S_{D}$ die Detektorfläche, $Q$ die Detektionseffizienz (typischerweise 0.5) und $V_{i}$ die Spannung bei der Ablösung des $i$-ten Atoms.

Die so rekonstruierten $z$-Positionen bilden allerdings nicht die von der Spitze abgetragene Struktur ab. Stattdessen ist eine Krümmung nach außen feststellbar. Dies liegt daran, dass Eckenatome zuerst evaporiert werden. Dadurch entsteht eine Verdampfungsreihenfolge, die von außen nach innen verläuft. Um diese Krümmung zu korrigieren, muss der Abstand $\sqrt{x^{2}+y^{2}}$ des Atoms vom Zentrum der Spitze berücksichtigt werden. Mit den bisherigen Annahmen kann man nun folgende Korrektur $z^{\prime}$ durchführen:

$$
z^{\prime}=R\left(1-\sqrt{1-\frac{x^{2}+y^{2}}{R^{2}}}\right) .
$$

Bas et al. [Bas1995] beschreiben, wie eine zusätzliche Korrektur der $(x, y)$-Koordinaten für den Fall erfolgt, dass die TAP-Analyserichtung nicht entlang der Spitzenachse, sondern verkippt dazu gewählt wird. In diesem Fall ist der bereits weiter oben erwähnte Parameter $m$ ungleich Null. Der Vergrößerungsfaktor $\eta$ sowie $K_{0}$ werden winkelabhängig. Diese Korrektur bewirkt allerdings meist nur geringe Verbesserungen und ist für die in dieser Arbeit durchgeführten Simulationen (Kap. 7) nicht nötig.

Es genügt für unsere Zwecke als Endergebnis die $z$-Komponente mit einer einfachen Korrektur zu ermitteln:

$$
\begin{aligned}
z & =z_{0}+z^{\prime} \\
& =\sum_{i=1}^{N-1} K_{0} / V_{i}^{2}+R\left(1-\sqrt{1-\frac{x^{2}+y^{2}}{R^{2}}}\right)
\end{aligned}
$$




\subsection{Das Feldionenmikroskop (FIM)}

Der experimentelle Aufbau des Feldionenmikroskops (FIM) ist nahezu identisch mit dem des gewöhnlichen TAP. Es wird jedoch auf eine Pulspannung verzichtet und zusätzlich ein Bildgas mit einem Druck im Bereich von $10^{-3}$ Pa in die Analysekammer eingelassen. Der Detektor wird durch eine Kombination aus Multikanalplatten, welche wiederum zur Signalverstärkung dienen, und einem Phosphorbildschirm ersetzt. Als Bildgase werden gewöhnlich Neon oder Helium sowie in seltenen Fällen Wasserstoff verwendet. Die Gasatome ionisieren bevorzugt an aus der Oberfläche herausragenden Stellen, da dort eine höhere Feldstärke vorliegt. Dies ist an den Rändern der Atomebenen in kristallographische Richtungen der Fall, welche die für eine nichtamorphe Probe typischen Ringe erzeugen. Ausscheidungen oder Cluster sowie Korngrenzen bedingen ebenfalls meistens eine Abweichung der lokalen Geometrie und lassen sich deswegen im FIM beobachten. Die Entscheidung, welches Bildgas verwendet wird, hängt von der Verdampfungsfeldstärke der untersuchten Probe ab. Im Gegensatz zum TAP lässt sich mit dem FIM ein stabiles Bild betrachten, indem die an der Spitze anliegende Spannung unterhalb der für die Feldverdampfung notwendigen Spannung eingestellt wird. Das Bildgas ist gut geeignet, wenn bereits ausreichend Gasatome ionisiert werden um ein Bild auf dem Schirm zu sehen. Das Feld, bei dem das Bild den besten Kontrast liefert, wird als Bestbildfeldstärke bezeichnet.

Das verwendete FIM ist in Göttingen im gleichen Aufbau untergebracht wie das TAP, lediglich der Abstand zum Detektor ist von $50 \mathrm{~cm}$ auf $5 \mathrm{~cm}$ reduziert. Dadurch ist ein wesentlich größerer Anteil der Oberfläche der FIM-Spitze sichtbar. Der Durchmesser des Phosphorschirms entspricht etwa $120^{\circ}$ (bestimmt für eine monokristalline Wolframspitze), wobei sich je nach Spitzenform, besonders für nicht monokristalline Spitzen, Abweichungen ergeben. Es bietet sich somit an, Spitzen zuerst im FIM zu untersuchen und dann so zu verkippen, dass sich der Bereich, den man im TAP messen möchte, in der Bildschirmmitte befindet. Ein FIM-Bild von Wolfram mit dem Vergleich zum Analysebereich des TAP ist in Abb. 6.15 in Kap. 6.4.4 zu sehen. 


\subsection{Das Transmissionselektronenemikroskop (TEM)}

Die in dieser Arbeit präsentierten Transmissionelektronenmiskroskopie (TEM)-Aufnahmen wurden an einem Tecnai F20 STEM bei einer Spannung von 200kV von Y.Yuan in Nanjing, China, erstellt. Zusätzlich wurde das Philipps CM12 mit 120kV in Göttingen gelegentlich zur Begutachtung der FIM-Spitzen verwendet. Interessant im Hinblick auf diese Arbeit ist, dass es möglich ist, mithilfe des TEM eine Bestimmung der Platzbesetzung durchzuführen. Die dafür verwendete Methode wird Atom Location By Channeling Enhanced Microanalysis (ALCHEMI) genannt. Zur Durchführung von ALCHEMI wird eine dünne Probe mit einer Dicke im Bereich von 100 nm benötigt, die auch für High Resolution TEM (HRTEM) geeignet ist. Die Probe muss so ausgerichtet sein, dass die Überstrukturrichtung senkrecht zur Richtung des Elektronenstrahls liegt. Nun wird entweder Energy Dispersive X-Ray (EDX) oder Electron Energy Loss Spectroscopy (EELS) durchgeführt. Mit dieser Methode lässt sich jeweils ein Signal der verschieden Komponenten, in unserem Fall TiAl und dem Zusatz, messen. Die Probe wird leicht verkippt und es wird erneut gemessen. Das Signal der drei Komponenten wird sich verändert haben, je nachdem an welchen Atomen das Channeling stattfindet. Nun kann man bestimmen, ob das Signal der zusätzlichen Komponente mit dem Signal von Ti oder von Al wächst. Wächst es mit dem Signal von Ti, so belegt der Zusatz Ti-Plätze, wächst er mit Al, so belegt er Al-Plätze. Durch Verwendung mehrerer Winkeleinstellungen lässt sich auch ein Anteil verschiedener Platzbesetzungen bestimmen. Da andere Gruppen bereits ausreichend Untersuchungen zur Platzbesetzung von ternären Elementen in $\gamma$-TiAl durchgeführt haben, wurden für diese Arbeit keine eigenen Versuche durchgeführt.

\subsection{Focused Ion Beam (FIB)}

Mit einem Focused Ion Beam (FIB) lassen sich FIM-Spitzen herstellen. Dafür wird mit einem fokussierten Gallium-Strahl aus dem Probenmaterial ein Steg freigeschnitten. Anschließend wird der Mikromanipulator mit dem Ende des Stegs durch Deposition von Platin verschweißst. Vom Steg wird nun ein Block mit möglichst quadratischer Grundfläche abgeschnitten. Dieser ist nun mit dem Mikromanipulator verbunden und wird vom Basismaterial entfernt. Diesen Vorgang nennt man Lift Out. Der Block wird nun auf eine vorgeätzte Wolframspitze, deren Oberfläche zuvor mit dem Galliumstrahl abgeflacht wurde, aufgesetzt und mittels Pt-Deposition befestigt. Anschließend wird die Verbindung zwischen Mikromanipulator und Spitze 
mit dem Galliumstrahl durchtrennt und der Mikromanipulator von der Spitze entfernt. Zuletzt schneidet man aus dem, nun auf der Wolframspitze stehenden, Block eine Spitze mit in etwa kreisförmiger Grundfläche heraus. Da ein Rasterelektronenmikroskop im Gerät integriert ist, lassen sich bei ausgeschaltetem Galliumstrahl Aufnahmen der Probe erstellen. Dieses Verfahren ist sehr ausführlich bei C. Wille [Wi112009] oder T. Kresse [Kres2009] beschrieben. Die Proben für diese Arbeit wurden überwiegend elektrolytisch hergestellt. 


\section{Kapitel 4}

\section{Analysealgorithmen}

Zur Durchführung der Analyse war es erforderlich, einige neue Algorithmen zu entwickeln und bereits bestehende Methoden für die Anforderungen der vorliegenden Daten anzupassen. Der Programmcode kann auf Anfrage vom Autor bereitgestellt werden. Zur Berechnung der Ausgabe wurde ein Dual-Xeon mit 2GB Arbeitsspeicher und Suse-Linux eingesetzt. Die 3D-Visualisierung erfolgte innerhalb der zur Auswertung von TAP-Daten verwendeten Software von AVS (Advanced Visual System).

\subsection{Bisherige Methoden zur Analyse der Kristall- struktur}

Ein Problem bei der Betrachtung von Atomsondendaten ist die starke Streuung der Atompositionen. Prinzipiell würde man erwarten, dass man Atompeaks verschiedener Elemente unterscheiden kann, wenn man Konzentrationsprofile oder Leiterdiagramme senkrecht zu erkennbaren Ebenen betrachtet. Tatsächlich ist dies prinzipiell möglich, wie etwa bei Kim et al. [Kim1997], der anhand von Konzentrationsprofilen von TiAl, sogar die Position von Chrom zuordnen kann. Leider funktioniert dies nur bei wenigen Messungen. Zudem ist die Interpretation des Konzentrationsverlaufs nicht unbedingt sinnvoll. Es kann gut sein, dass die Ebenenabfolge dadurch zustande kommt, dass die Streuung eines Elements größer ist als die des anderen. Dieser Zusammenhang ist in Abb. 4.1 dargestellt. Man kann somit eine scheinbare Abfolge verschieden zusammengesetzter Ebenen auch für nicht geordnete Richtungen feststellen. 

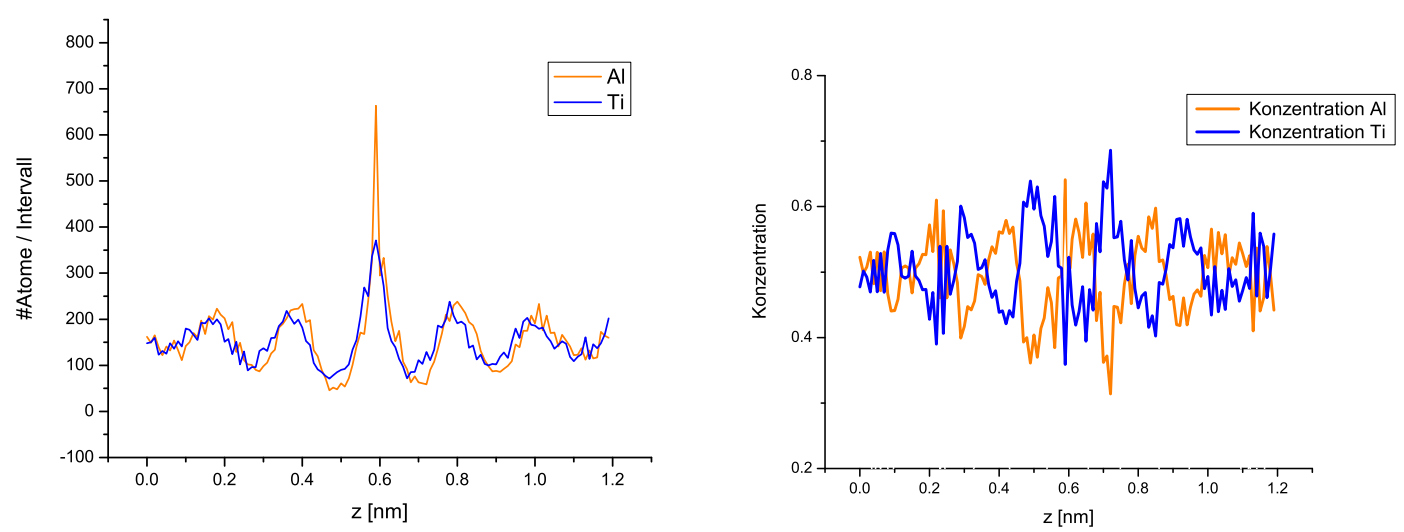

Abbildung 4.1: Dichteprofil (links) und das zugehörige Konzentrationsprofil (rechts) im Vergleich. Die Profile sind AtomVicinity-Dichteprofile, die später in Kap. 4.2 eingeführt werden. Prinzipiell kann man aber auch mit unbearbeiteten Atomsondendaten vergleichbare Diagramme erstellen. Die Datenbasis sind simulierte Atomsondendaten für die [100]Richtung in TiAl. Es liegen im Abstand von etwa $0.2 \mathrm{~nm}$ gemischte Ebenen vor. Dass dies der Fall ist, erkennt man in den Dichteprofilen korrekt. Betrachtet man hingegen Konzentrationsprofile, so könnte man sich abwechselnde Ti- und Al-Ebenen im Abstand von 0.1 nm vermuten.

Besser geeignet erscheint eine statistische Untersuchung anhand der Dichte. Von Vurpillot et al. [Vurp2001b] wurden Fourier-Analysen durchgeführt. Dieses Vorgehen ist allerdings für die Analyse von intermetallischen Phasen, deren Kristallgitter in der TAP-Messung weit stärker verrauscht ist als das von einfachen Kristallen, zu rechenaufwändig, weil die erforderliche Anzahl von Atomen zu groß ist. Für diese Arbeit wurde ein Ansatz gewählt, der dadurch, dass die Ergebnisse im Realraum bleiben, einen intuitiveren Zugang bietet.

\subsection{AtomVicinity}

Der verwendete AtomVicinity-Algorithmus wurde im Wesentlichen bereits in der Diplomarbeit von T. Boll [Boll2005] vorgestellt. Eine wesentliche Verbesserung erfolgte allerdings in der Leistungsfähigkeit. So ist nun einerseits ein paralleles Rechnen auf mehreren Prozessorkernen möglich, andererseits wurde auch die Effizienz durch eine Optimierung des Programmcodes verbessert. Das generelle Funktionsprinzip ist allerdings gleich geblieben und wird bildlich in Abb. 4.2 erläutert. Der prinzipielle Programmablauf ist zudem in einem Nassi-Schneidermann-Diagramm in Abb. 4.3 dargestellt. Nassi-Schneidermann-Diagramme lassen sich auch ohne tiefergehende 

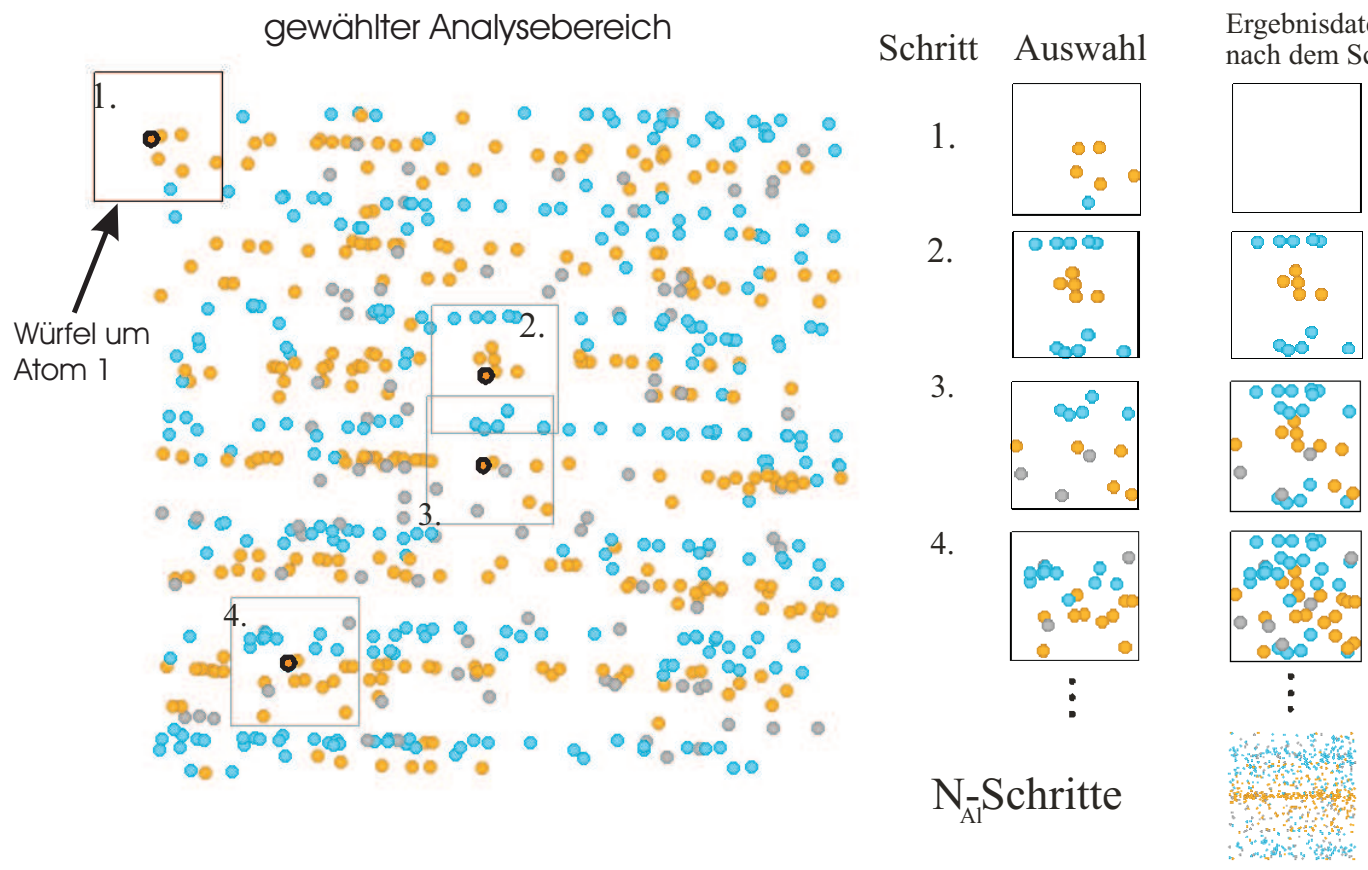

Abbildung 4.2: Prinzip von AtomVicinity: Um die links im Analysebereich befindlichen orangefarbenen Al-Referenzatome herum wird eine Umgebungsanalyse ausgeführt. Im 1. Schritt (rechts oben) wird die Umgebung um das Referenzatom 1 (links dick umrandet) nicht für die Ausgabe verwendet, da es teilweise außerhalb des Volumens liegt. Im 2. Schritt wird das Referenzatom inklusive Umgebung in die Ausgabe kopiert. Dies wiederholt sich im 3. Schritt. Hierbei ist anzumerken, dass nun Atome, die sich bereits in der Umgebung des Referenzatoms aus Schritt 2 befanden, erneut in die Ausgabe kopiert werden. Im 4. und allen folgenden Schritten vergrößert sich die Ausgabe, bis schließlich nach $N_{A l}$-Schritten, also dem Durchlaufen aller orangen Atome, ein Ergebnisdatensatz vorhanden ist, der eine deutlich bessere Unterscheidung der Netzebenen ermöglicht.

Kenntnisse meist intuitiv verstehen. Bei weiterem Interesse ist die Einführung von Cormen [Corm2004] empfehlenswert.

Im Grunde erlaubt der Algorithmus die Darstellung der typischen Umgebung eines Referenzatoms. Eingabe und Ausgabe des Algorithmus sind 3D-Atomdaten. Bevor der Algorithmus beginnt, muss ein quaderförmiges Volumen aus der gesamten TAP-Messung herausgeschnitten werden. Dieses Volumen bezeichnen wir als Analysevolumen oder Analysebereich. Nun muss der Typ $A$ der Referenzatome ausgewählt werden. Dies sind üblicherweise alle, die demselben Element zugeordnet werden können. Im Beispiel in Abb. 4.3 ist Aluminium gewählt. Anschließend wird der Typ der Analyseatome $B$ gewählt. Im Beispiel sind dies Al, Ti und Nb. Zusätzlich muss eine Kantenlänge $2 l$ ausgewählt werden, die die Abmessungen des Auswahlwürfels vorgibt. Nun wird für jedes Referenzatom $a \in A$ das Volumen innerhalb des Auswahlwürfels mit der Kantenlänge $2 l$ betrachtet. Die relativen Atompositionen zu $a$ 
AtomVicinity2 - Algorithmus zur Analyse der Nahordnung

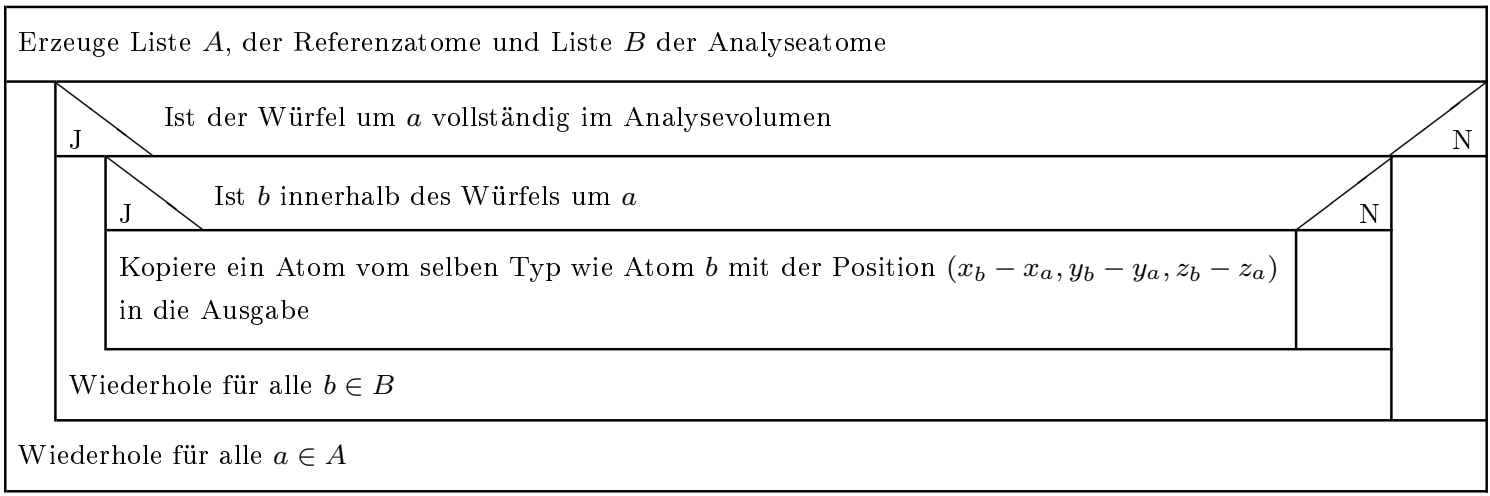

Abbildung 4.3: Struktogramm des AtomVicinity-Algorithmus. Falls eine parallele Berechnung stattfinden soll, so wird $A$ in $n$ Mengen $A_{1} \ldots A_{n}$ aufgeteilt und es wird in $n$ Threads mit $A_{i}$ im $i$-ten Thread $((i \in[1 . . n]))$ anstelle von $A$ gerechnet.

jedes Analyseatoms $b \in B$ innerhalb des jeweiligen Volumens werden in die Ausgabe kopiert. Die neue Position eines solchen Atoms $b$ ist also $\left(x_{b}-x_{a}, y_{b}-y_{a}, z_{b}-z_{a}\right)$. Lediglich für Referenzatome, deren Würfel teilweise außerhalb des Analysevolumens liegen, geschieht dies nicht.

Die Laufzeit ist demzufolge nun von der Größenordnung $\mathcal{O}\left(N_{\text {Referenz }} \cdot N_{\text {Analyse }}\right)$, wobei $N_{\text {Referenz }}$ die Anzahl der gewählten Atome ist, für die die Umgebungsanalyse durchgeführt werden soll. $N_{\text {Analyse }}$ ist die Zahl der Atome, deren Position untersucht werden soll. Zuvor war die Laufzeit $\mathcal{O}\left(N_{\text {Gesamt }}^{2}\right)$ mit der Gesamtzahl $N_{\text {Gesamt }}$, der im Analysevolumen liegenden Atome $\left(N_{\text {Gesamt }}>N_{\text {Analyse }}\right.$ und $\left.N_{\text {Gesamt }}>N_{\text {Referenz }}\right)$, größer. Für eine Analyse mit 0.5 Mio. Referenzatomen und 1 Mio. Analyseatomen benötigt das System bei direkter Ausgabe von Dichtekarten ohne Parallelisierung etwa einen Tag. Kleine Volumen mit $<50 \mathrm{k}$ Atomen lassen sich bei paralleler Berechnung in wenigen Minuten berechnen.

Die "Spatial Distribution Maps", die von Geiser et al. [Geis2007] unabhängig (von [Boll2005]) entwickelt wurden, arbeiten weitgehend identisch. Der einzige Unterschied ist, dass die Randwürfel nicht aussortiert werden, was zusätzliche Artefakte verursacht, die allerdings korrigierbar sind [Mood2009]. Der andere Unterschied ist, dass das Zentralatom nicht für die Ausgabe mitberücksichtigt wird. Dadurch verschwindet zwar die in der Mitte der Ausgabe enthaltene hohe Punktedichte des Referenzatoms. Die mittlere Dichte der Referenzatome wird dadurch allerdings als zu gering angegeben.

Prinzipiell ermöglicht unser Algorithmus die Betrachtung der durchschnittlichen 

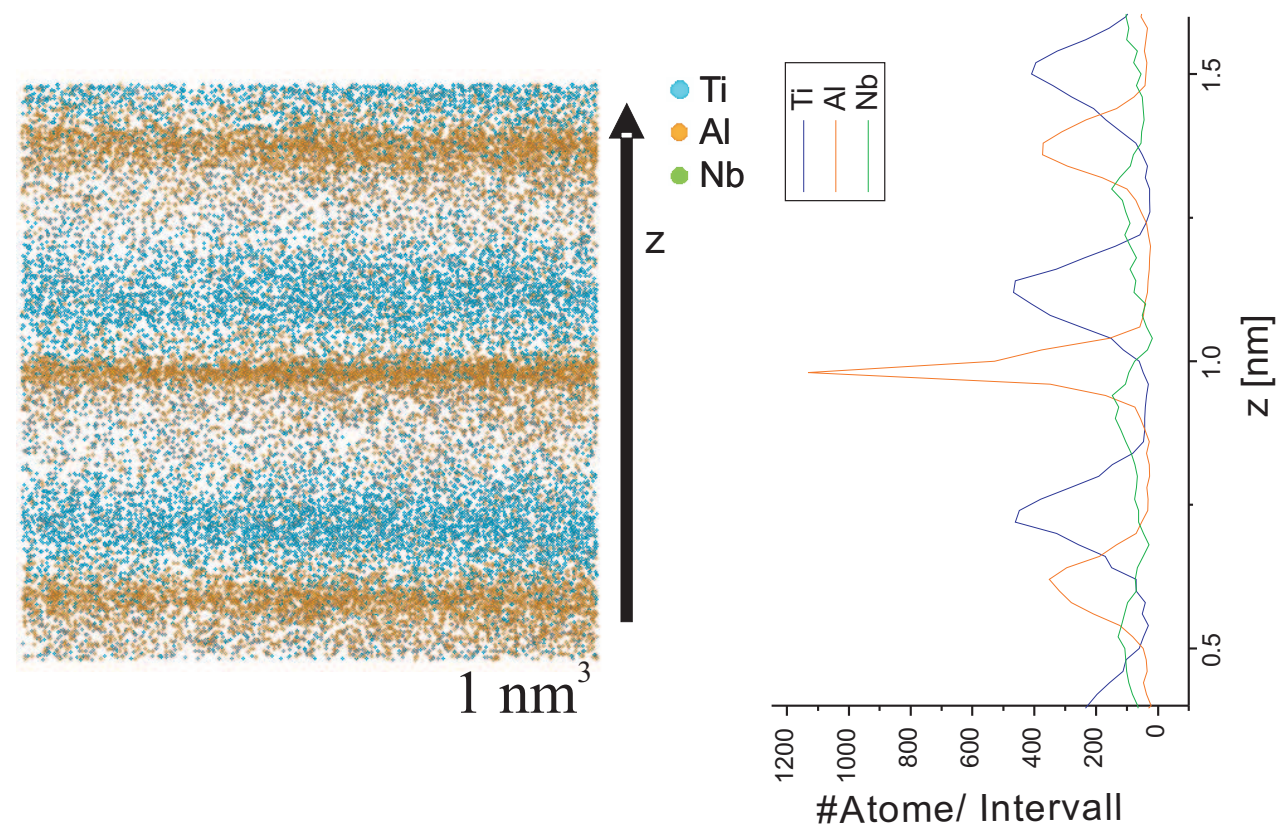

Abbildung 4.4: Aus den Ergebnissen von AtomVicinity (links) lassen sich Dichteprofile (rechts) erstellen. Die Daten wurden vor der Durchführung von AtomVicinity so verkippt, dass die Ebenen senkrecht zur z-Achse stehen. Bereits links kann man die Netzebenen erkennen und qualitativ die Positionen von Al- und Ti-Ebene. Anhand der Dichteprofile ist eine quantitative Aussage möglich. Zudem sind auch die Positionen der Nb-Atome erkennbar.

Umgebung eines Elements in drei Dimensionen. Die Ergebnisse lassen sich nun einerseits als Schnitte in Form von Flächendichteprofilen verwerten, andererseits können auch Liniendichteprofile erstellt werden, wie in Abb. 4.4 demonstriert wird. Letztere sind für Richtungen senkrecht zu sichtbaren Netzebenen besonders interessant, da sie gut vergleichbare quantitative Aussagen ermöglichen und mit dem meist verwendeten TAP ohnehin keine ausreichende Auflösung in $(x, y)$-Richtung erzielt werden kann. Sofern es nicht anders angegeben ist, ist die Intervallbreite innerhalb dieser Dichteprofile $0.01 \mathrm{~nm}$.

\subsection{Kreuzkorellation}

Um auch in schwierigen Fällen mit hohem Rauschen einen Vergleich der Ebenenpeaks der Dichteprofile zu ermöglichen wurde eine Kreuzkorrelationsmethode verwendet. Durch Kreuzkorrelation lassen sich zwei AtomVicinity-Dichteprofile zudem leichter automatisch vergleichen als durch eine Bestimmung der Peakpositionen. Die Kreuzkorellationsfunktion zweier auf eine mittlere Dichte von 0 normierte Dichte- 
profile $^{1} a(z)$ und $b(z)$ im Intervall $\left[-z_{\max }, z_{\max }\right]$ ist definiert als

$$
R_{a, b}(\Delta z)=\lim _{z_{\max } \rightarrow \infty} \int_{-z_{\max }}^{z_{\max }}(a(z)-\bar{a}) \cdot(b(z+\Delta z)-\bar{b}) d z .
$$

Hierbei sind $\bar{a}=\lim _{z_{\max } \rightarrow \infty} \int_{-z_{\max }}^{z_{\max }} a(z) / 2 z_{\max }, \bar{b}=\lim _{z_{\max } \rightarrow \infty} \int_{-z_{\max }}^{z_{\max }} b(z) / 2 z_{\max }$ die Mittelwerte der Funktionen. Wird die Signalverschiebung $\Delta z$ für zwei AtomVicinityDichteprofile des gleichen Materials in die gleiche kristallographische Richtung so gewählt, dass $R_{a, b}$ maximal wird, so kann $\Delta z$ mit dem Ebenenabstand identifiziert werden. Der erhöhte Mittelpeak wird, obwohl er für Artefakte sorgt, in die Berechnung mit einbezogen, da seine Entfernung ebenfalls Artefakte verursachen würde. Um die Vergleichbarkeit der Ergebnisse zu verbessern, wird die Korrelation normiert. Die tatsächliche Berechnung erfolgt für die Verschiebung um $j \in\left[j_{\min }, j_{\max }\right] \subset \mathbf{Z}$, $j_{\text {min }} \leq 0, j_{\text {max }} \geq 0$ Intervalle mit $N=$ Gesamtzahl der Intervalle über

$$
\begin{aligned}
& R_{a, b}(j)= \frac{\sum_{i=1}^{N}(a(i)-\bar{a}) \cdot\left(b^{\prime}(i+j)-\overline{b^{\prime}}\right)}{\sum_{i=1}^{N}(a(i)-\bar{a})^{2} \sum_{i=1}^{N}\left(b^{\prime}(i+j)-\overline{b^{\prime}}\right)^{2}} \\
& \operatorname{mit} b^{\prime}(i+j)=\left\{\begin{array}{l}
b(i+j) \text { für } i+j \leq j_{\text {max }}, i+j \geq j_{\text {min }} \\
0 \text { sonst }
\end{array}\right.
\end{aligned}
$$

Der Vorteil der Kreuzkorrelation ist, dass auch bei sehr verrauschten Signalen, die keine Gaussfits mehr erlauben, immer noch eine Analyse durchgeführt werden kann, die zudem nicht nur die Werte im Bereich der Peaks, sondern fast die ganze Messung mit einbezieht. Problematisch ist, dass die endlichen Intervallbreiten die Auflösung dieser Methode beschränken. Zudem ist ein quantitativer Vergleich bei kleinen zusätzlichen Peaks schwierig. Deswegen wurde diese Methode nur eingesetzt, wenn anderweitig eine Auswertung nur unzureichend möglich war. Der Effekt dieser Berechnung wird in Abb. 4.5 demonstriert.

\footnotetext{
${ }^{1}$ Die Überladung der Bedeutung von $a$ und $b$, die neben einer Atomdichte auch einzelne Atome bedeuten können, ist nicht unbeabsichtigt.
} 

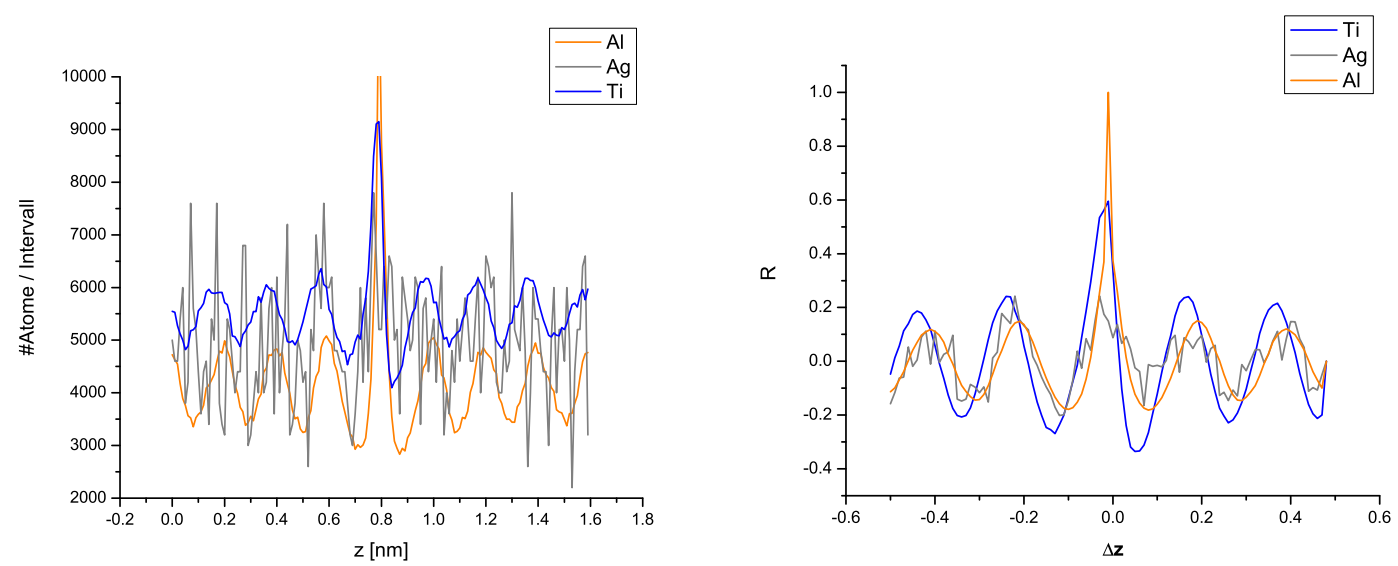

Abbildung 4.5: Ti46.4at\%Al0.2at.\%Ag in [100]-Richtung: Im normalen AtomVicinityDichteprofil (links) mit Al als Referenzatom lassen sich Ti- und Al-Peaks bereits gut unterscheiden. Eine Aussage über die Positionen des Ag-Peaks ist hingegen nicht möglich. Auch Gauss- sowie viele andere Fits liefern keine Erkenntnis über die genaue Peakposition. Nach Ausführen der Kreuzkorrelation der jeweiligen Komponenten zu Al (rechts) ist die Bestimmung der Peak-Position für alle Komponenten möglich. Die Reihenfolge der Peaks ist Ti-Ag-Al. 


\section{Kapitel 5}

\section{Probenpräparation}

Es wurden Proben mit den folgenden nominellen Zusammensetzungen (in at.\%) untersucht:

1. $\mathrm{Ti}_{51} \mathrm{Al}_{48} \mathrm{Nb}_{1}$

2. $\mathrm{Ti}_{47} \mathrm{Al}_{48} \mathrm{Nb}_{5}$

3. $\mathrm{Ti}_{42} \mathrm{Al}_{48} \mathrm{Nb}_{10}$

4. $\mathrm{Ti}_{46} \mathrm{Al}_{52} \mathrm{Ag}_{2}$

5. $\mathrm{Ti}_{52} \mathrm{Al}_{45} \mathrm{Cr}_{3}$

6. $\mathrm{Ti}_{54} \mathrm{Al}_{45} \mathrm{Cr}_{1}$

7. $\mathrm{Cu}_{3} \mathrm{Au}$

8. $\mathrm{Ni}_{92} \mathrm{Al}_{8}$

9. $\mathrm{Al}_{92} \mathrm{Li}_{8}$

Die TiAl-Legierungen wurden durch Levitationsschmelzen an der Nanjing University in Nanjing, China, hergestellt. Dafür wurden die Proben dreimal aufgeschmolzen um eine bessere Durchmischung des Materials zu gewährleisten. $\mathrm{Cu}_{3} \mathrm{Au}$ wurde von der Fa. Wieland in Drahtform bezogen. $\mathrm{Ni}_{92} \mathrm{Al}_{8}$, welches als Referenzmaterial benutzt wurde, wurde in Göttingen hergestellt. $\mathrm{Al}_{92} \mathrm{Li}_{8}$ wurde vor $20 \mathrm{Jahren}$ von den Vereinigten Aluminium Werken (VAW) bereitgestellt [AK1992]. 
$\mathrm{Cu}_{3} \mathrm{Au}$ wurde zunächst 30 Tage bei $823 \mathrm{~K}$, also oberhalb der Ordnungstemperatur ausgelagert. Danach wurde die Probe weitere 90 Tage bei $623 \mathrm{~K}$, also unterhalb der Ordnungstemperatur wärmebehandelt. Das anschließende Abkühlen auf $473 \mathrm{~K}$ erfolgte langsam im Ofen über den Zeitraum von 7 Tagen.

Die TiAl-Proben erhielten unterschiedliche Wärmebehandlungen. Abgesehen von $\mathrm{Ti}_{46} \mathrm{Al}_{52} \mathrm{Ag}_{2}$ wurden sie allerdings auch "as cast" verwendet. $\mathrm{Ti}_{46} \mathrm{Al}_{52} \mathrm{Ag}_{2}$ wurde $10 \mathrm{~h}$ bei $1473 \mathrm{~K}$ und anschließend $60 \mathrm{~h}$ bei $1273 \mathrm{~K}$ ausgelagert und anschließend in Wasser abgeschreckt. Die Wärmebehandlung der Referenzmaterialien ist unbekannt.

Sämtliche Wärmebehandlungen fanden in Quarzglasröhrchen unter einer ArgonSchutzgasatmosphäre statt.

\subsection{Präparation für FIM und TAP}

Aus den herzförmigen Barren wurden mit Funkenerosion Quader mit einer etwa quadratischen Grundfläche von $200 \times 200 \mu \mathrm{m}^{2}$ geschnitten. Einige der TiAl-Proben wurden danach bei $1273 \mathrm{~K}$ für $44 \mathrm{~h}$ ausgelagert, um das Material zu erholen und die Wahrscheinlichkeit eines Abbruchs der Spitzen während einer TAP- oder FIMMessung so gering wie möglich zu halten. Dies wurde nicht für alle Proben durchgeführt, da geringere Ordnungsgrade die TAP-Analyse erleichtern (Kap. 7.6.7). Nicht ausgelagerte Spitzen wurden deshalb ebenfalls verwendet.

Für die Feldionenmikroskopie wurden die Stäbe elektrolytisch zu geeigneten Spitzen gedünnt. Dies geschah meist in zwei Schritten. Zuerst bildete sich bei Gleichspannungen von 30 bis $10 \mathrm{~V}$ ein schmaler Hals aus. Anschließend wurde bei einer niedrigeren Spannung von $5 \mathrm{~V}$ pulsweise geätzt bis der Hals durchtrennt war. Für alle Materialien außer $\mathrm{Cu}_{3} \mathrm{Au}$ war 95 vol\% Essigsäure mit 5 vol\% Perchlorsäure der verwendete Elektrolyt. Auch höhere Perchlorsäureanteile und Elektrolyten mit Methanol wurden getestet, führten aber zu schlechteren Ergebnissen. Abschließend fand eine Reinigung der Spitzen in Methanol statt. $\mathrm{Cu}_{3} \mathrm{Au}$ wurde mit einer Elektrolytlösung von $10 \mathrm{~g} \mathrm{Na}_{2} \mathrm{Cr}_{4} \mathrm{O}_{7}$ in $50 \mathrm{ml}$ 95vol.\% Essigsäure mit derselben Methode wie die anderen Proben bei Gleichspannung produziert.

In dieser Arbeit wurde zum Spitzendünnen nicht die übliche Methode angewandt, durch periodisches Auf- und Abbewegen des Probendrahtes innerhalb einer U-förmig gebogenen Flächenelektrode, Wirbel auszunutzen, die eine stärkere Abtragung in einem bestimmten Bereich und somit die Ausbildung einer Einschnürung bewirken. 
Stattdessen wurde, wie bereits in [Boll2005] vorgestellt, eine runde Elektrode benutzt. Dabei entstand ein stabiler Strömungswirbel, der ein einfaches Erstellen von Spitzen größerer Länge mit weniger Bewegung ermöglichte.

Probleme bei der Präparation der TiAl-Proben ergaben sich durch einen dünnen Film sowie eine poröse Schicht, die beim Dünnen entstanden. Diese Schicht wurde durch kurze Spannungspulse höherer Spannung (25-50V) entfernt, falls heftiges Aufund Abbewegen nicht half. War die Spitze schon weit entwickelt, so reichte dies meist, um die Schicht abzulösen. Bei der Präparation von $\mathrm{Ti}_{46} \mathrm{Al}_{52} \mathrm{Ag}_{2}$ brach die Spitze sehr häufig ab. Deswegen wurde, da die hohe Ausschussrate die Vorräte stark reduziert hatte, für dieses Material bei einigen Proben das FIB (Kap. ch:GrundlagenFIB) zur Herstellung verwendet.

\subsection{Präparation für TEM}

Die für die Transmissionselektronenmikroskopie bestimmten $\mathrm{TiAl}+\mathrm{Nb}-$ Proben wurden bei $1273 \mathrm{~K}$ für verschiedene Zeiten ausgelagert. Für $\mathrm{Ti}_{46} \mathrm{Al}_{52} \mathrm{Ag}_{2}$ waren dies $0 \mathrm{~h}$, 1h, 4h, 17h, $65 \mathrm{~h}$ bei $1073 \mathrm{~K}$. Die TiAl+Nb-Proben wurden für $44 \mathrm{~h}$ ausgelagert.

Mittels Funkenerosion wurden aus den Herzförmigen Rohlingen Scheiben geschnitten. Von zunächst $0.4 \mathrm{~mm}$ wurde die Dicke dieser Scheiben durch Schleifen und Dimpeln auf 20-50 $\mu \mathrm{m}$ reduziert. Abschließend wurde das Probenmaterial in einer Lösung von 93 vol.\% Ethanol und 7 vol.\% Perchlorsäure mittels des twin-jet electro polishing an einer Stelle bis auf die für TEM benötigte Dicke abgetragen.

\subsection{Präparation für X-Ray}

Die $\mathrm{Ti}_{54} \mathrm{Al}_{45} \mathrm{Cr}_{1}$-Proben für die Röntgenanalyse wurden durch Funkenerosien als kleine Blöcke mit den Abmessungen 1.5x1.0x0.2 $\mathrm{cm}^{3}$ hergestellt und anschließend abgeschliffen. Kurze $\mathrm{Cu}_{3} \mathrm{Au}$-Drähte wurden dicht nebeneinander auf einen Probenhalter geklebt, was natürlich Korrekturen bei den erhaltenen Spektren erforderte. 


\section{Kapitel 6}

\section{Experimentelle Ergebnisse}

\subsection{TEM-Ergebnisse}

Zur allgemeinen Charakterisierung der TiAl-Proben wurden TEM-Aufnahmen an einem Tecnai F20 bei 200 kV erstellt, anhand derer man die Mikrostruktur erkennen kann. Diese sind vor allem für die $\mathrm{Ti}_{46} \mathrm{Al}_{52} \mathrm{Ag}_{2}$-Proben interessant, da dort die ansonsten in allen Proben vorhandene, und nach [Boll2005] erwartete, Lamellenstruktur nicht auftritt. Abgesehen von den TEM-Aufnahmen der FIM-Spitzen, die am Philipps CM12 mit $120 \mathrm{kV}$ produziert wurden, stammen die TEM-Untersuchungen von Yong Yuan.

\subsection{1 $\mathrm{TiAl}+\mathrm{Nb}$}

$\mathrm{TiAl}+\mathrm{Nb}$ wurde bereits in [Boll2005] charakterisiert. In den entsprechenden TEMAufnahmen (Abb. 6.1) lässt sich für alle Proben eine $\mathrm{D} 0_{19^{-}} \gamma / \alpha_{2}$-Lamellenstruktur finden. Der Anteil der $\mathrm{L}_{0^{-}} \gamma$-Phase ist offenbar wesentlich größer als der der $\alpha_{2^{-}}$ Phase. Domänenengrenzen, wie in Abb. 6.1(b), können bei passender Orientierung im TAP gut beobachtet werden, da die Feldverdampfungsfeldstärken in verschiedene Richtungen unterschiedlich sind und an der Grenze somit ein lokaler Vergrößerungseffekt stattfindet. Dasselbe gilt für die $<110>$-Zwillinge der $\gamma$-Phase. In Abb. 6.1(d) ist eine HRTEM-Aufnahme zu sehen, die den Übergang einer $\alpha_{2}$-Ebene in eine $\gamma$ Ebene zeigt. Der Orientierungszusammenhang der beiden Phasen wurde im Rahmen dieser Arbeit als $(1 \overline{1} 1) \gamma / /(0001) \alpha_{2}$ mit $[101] \gamma / /[2 \overline{110}]$ anhand von Beugungsbildern bestimmt [AK2007]. 


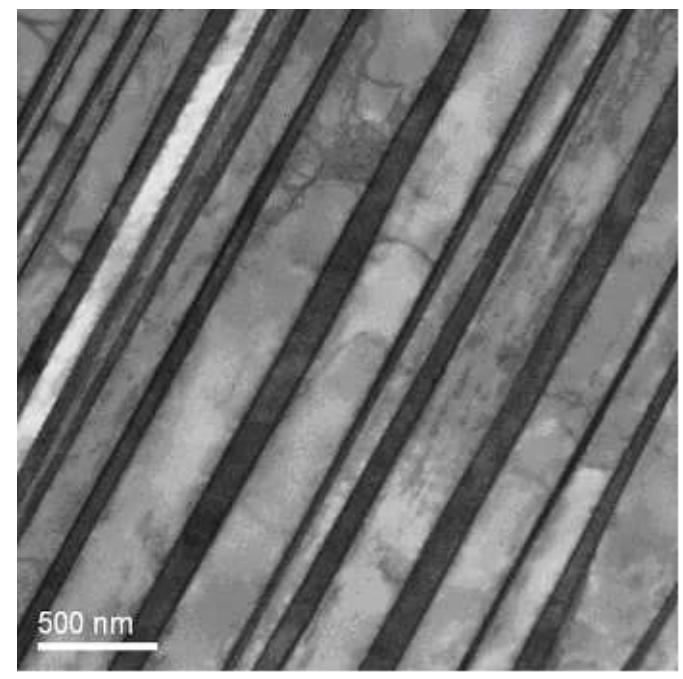

(a) $\mathrm{Ti}_{51} \mathrm{Al}_{48} \mathrm{Nb}_{1}$

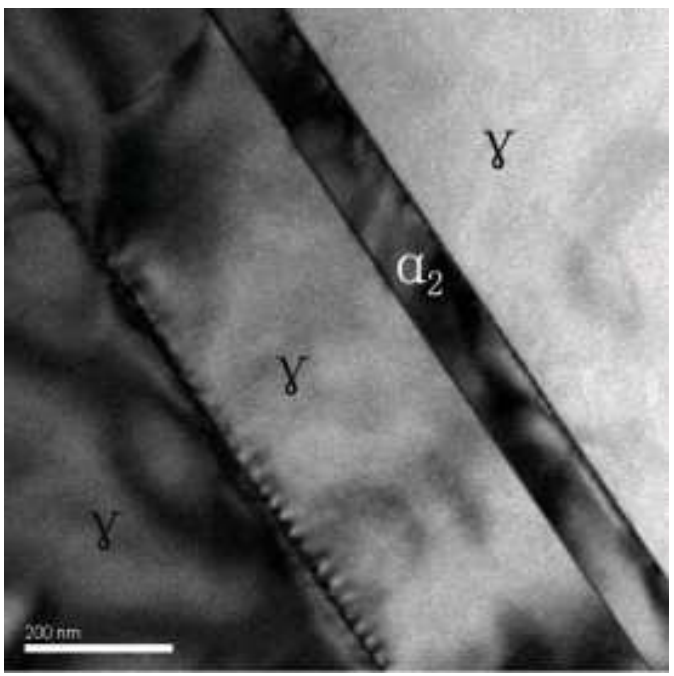

(c) $\mathrm{Ti}_{47} \mathrm{Al}_{48} \mathrm{Nb}_{5} \gamma / \gamma$-Zwillingsgrenze

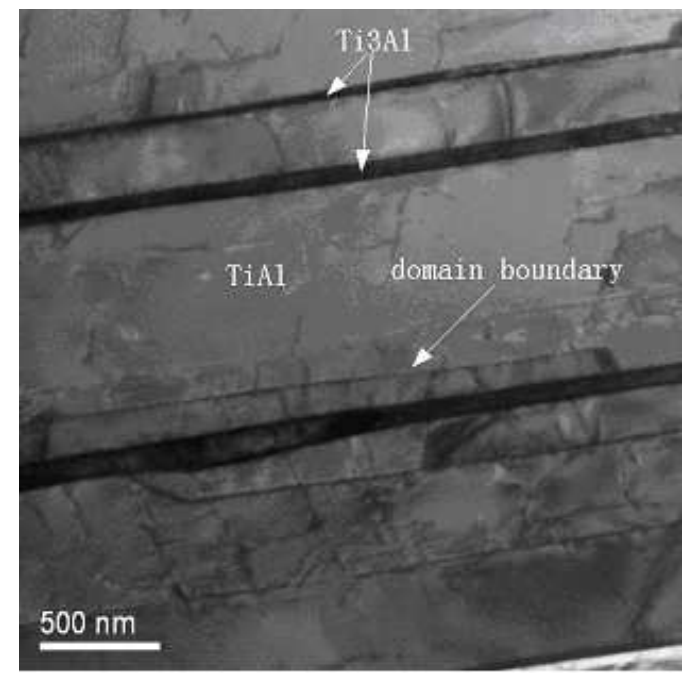

(b) $\mathrm{Ti}_{42} \mathrm{Al}_{48} \mathrm{Nb}_{10}$

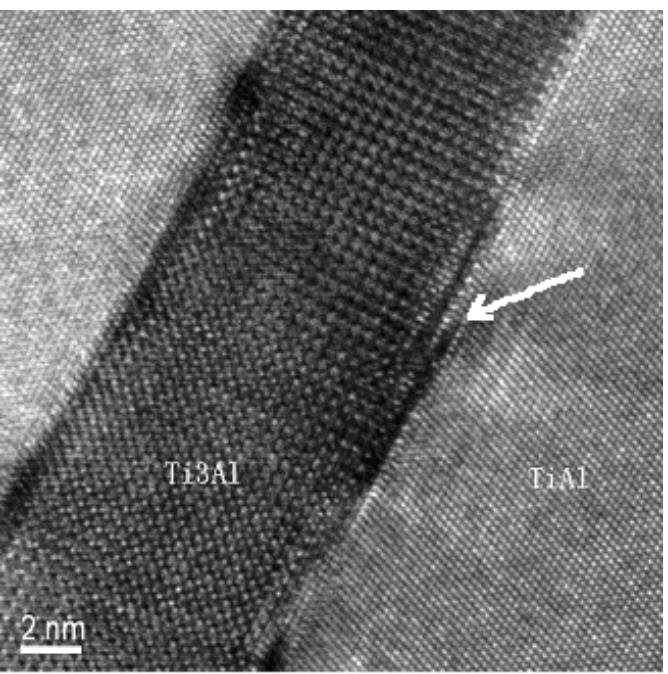

(d) $\mathrm{Ti}_{51} \mathrm{Al}_{48} \mathrm{Nb}_{1}$ HRTEM

Abbildung 6.1: TEM-Aufnahmen der nichtausgelagerten TiAl+Nb-Proben. In (a) ist eine $T i_{51} A l_{48} N b_{1}$-Probe abgebildet. Die $\gamma / \alpha_{2}$-Lamellenstruktur ist ebenso offensichtlich wie in (b) für $T i_{42} A l_{48} N b_{10}$ und in (c) für $T i_{47} A l_{48} N b_{5}$. In (b) ist zudem eine Domänengrenze zu erkennen. Abb. (c) zeigt eine $\langle 110>-Z$ willingsgrenze in der $\gamma$-Phase. In (d) ist eine HRTEM-Aufnahme zu sehen, die die Situation an einer $\alpha_{2} / \gamma$-Grenze darstellt. Mit dem Pfeil ist eine Stelle markiert, an der Halbebenen enden. 
In Abb. 6.2 sind spitzenförmige Proben zu sehen, wie sie für FIM und TAP verwendet werden. Man erkennt, dass die Spitze keineswegs eine perfekte Halbkugel ist. Zudem ist eine Spitze nicht mehr symmetrisch sobald asymmetrisch verteilte Körner auftauchen (Abb. 6.2(b)).

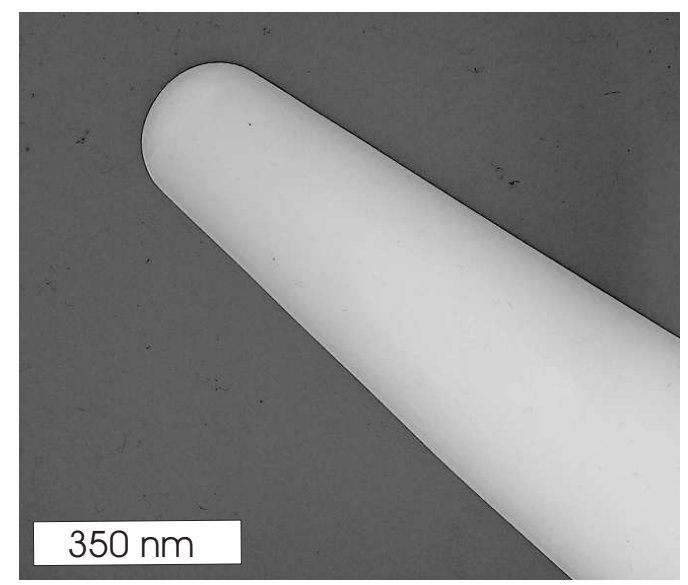

(a) reine $\gamma$-Phase

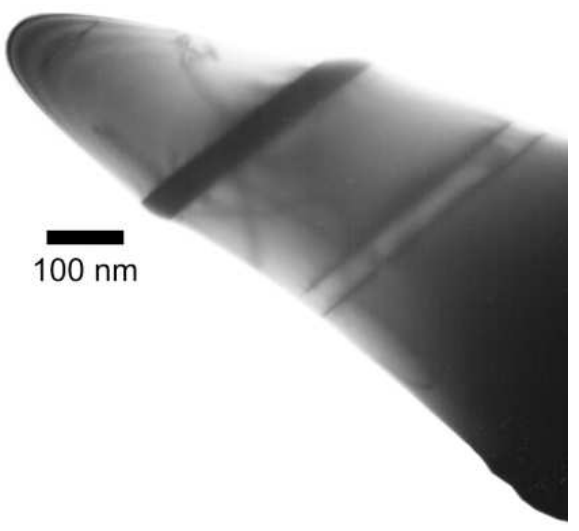

(b) lamellare Struktur

Abbildung 6.2: In (a) sieht man die TEM-Aufnahme einer $T i_{51} A l_{48} N b_{1}$-Probe nach einer TAP-Messung. Die $\gamma$-Phase erstreckt sich über die ganze Spitze. In (b) offenbart sich für eine $\mathrm{Ti}_{47} \mathrm{Al}_{48} \mathrm{Nb}_{5}$-Probe eine lamellare Struktur. Diese Struktur war nach einiger Feldverdampfung im FIM aber vor einer TAP-Messung sichtbar. An der Spitze liegt die $\gamma-$ Phase vor, gefolgt von einer dunkler abbildenden $\alpha_{2}$-Lamelle. Der negative Einfluss einer Mehrphasenstruktur auf die Zylindersymmetrie der Spitze lässt sich an den den Positionen der dünnen Lamellen beobachten.

Auch $\mathrm{Ti}_{52} \mathrm{Al}_{45} \mathrm{Cr}_{3}$ zeigt eine $\alpha_{2} / \gamma$-Lamellenstruktur. Da dies bereits im TAP und FIM gut zu erkennen ist, wird an dieser Stelle auf weitere TEM-Bilder dieser Proben verzichtet.

\subsubsection{TiAlAg}

Unter dem TEM offenbart sich, dass das Hinzulegieren von Silber die Ausbildung der $\alpha_{2} / \gamma$-Lamellenstruktur unterbindet. Statt dessen lassen sich große Körner der $\gamma$-Phase erkennen, in denen sich durch eine Temperaturbehandlung kleine Ag-reiche $L 1_{2}$-Ausscheidungen befinden. Die Art der vorliegenden Phase ließ sich dabei mit Hilfe von Beugungsbildern bestimmen. Diese Veränderung der Mikrostruktur erklärt 
die erschwerte Präparation der silberhaltigen Proben, welche eine hohe Sprödigkeit aufwiesen. Als Resultat waren bei elektrolytischer Präparation nur 5-10\% der Versuche erfolgreich. Bei einer weiteren Auslagerung bei hoher Temperatur wuchsen diese Ausscheidungen und verschlimmerten diese Materialeigenschaften zusätzlich, weshalb die TAP-Messungen nur an Proben ohne zusätzliche Wärmebehandlung durchgeführt werden konnten. In den TAP und FIM-Untersuchungen der nicht wärmebehandelten Proben wurden keine Ag-Cluster oder $\mathrm{L}_{2}$-Ausscheidungen gefunden. Es wurde allerdings auch nicht explizit danach gesucht. In Abb. 6.4 sind HRTEM-Aufnahmen der $\mathrm{L1}_{2}$-Ausscheidungen zu sehen. Bei größeren Auslagerungszeiten wird die unterschiedliche Gitterkonstante der Ausscheidungen in die nicht geordneten [100]-Richtungen der Matrix deutlich. In die (in der Matrix geordnete) [001]-Richtung stimmen die Gitterkonstanten noch mit der der Ausscheidung überein Dies kann man in den inversfouriertransformierten (IFFT -Inverse Fast Fourier Transformation) Bildern in Abb. 6.4(b) und 6.4(d) sehen.

\subsection{Röntgenanalyse}

\subsection{1 $\mathrm{TiAl}+\mathrm{Cr}$}

Da in den TiAl-Proben zwei unterschiedliche Phasen zu finden sind, ergeben die Röntgenanalysen eine Überlagerung zweier Beugungsbilder. Die Signalstärke ist relativ gering. Dies erklärt sich, wenn man berücksichtigt, dass die Körner als Lamellen vorliegen. Für den großen Anteil der Lamellen, die senkrecht zur Strahlrichtung liegen, erhält man, aufgrund der Lamellendicke im Bereich weniger 100 nm, "Peaks" mit erheblicher Breite. Aus dem geringen Anteil der Lamellen, die in Strahlrichtung liegen, erhält man die scharfen Peaks, wie in Abb. 6.5 zu sehen. Die Angabe einer Korngröße nach Scherrer ist somit von geringem Wert.

Auch die Bestimmung des Ordnungsparameters $s \in[0,1]$ über einen einfachen Vergleich der Intensitäten $I$ der Peaks in Überstrukturrichtung mit den zugehörigen Peaks des nicht geordneten Materials wird durch diese Einflüsse erschwert. Dies ist durchaus vergleichbar mit den Problemen, die bei J.Chen et al. [Chen2003] bei dünnen $\mathrm{CuAu}-\mathrm{Schichten}$ auftreten. 


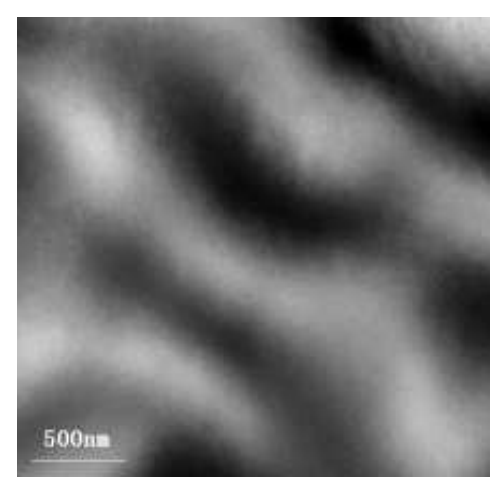

(a) 0h, [001]

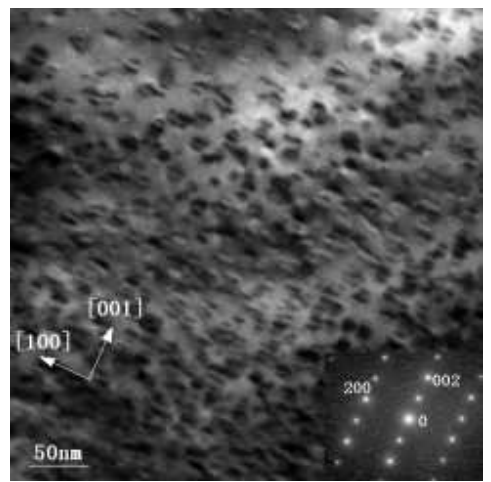

(b) $1 \mathrm{~h},[010]$

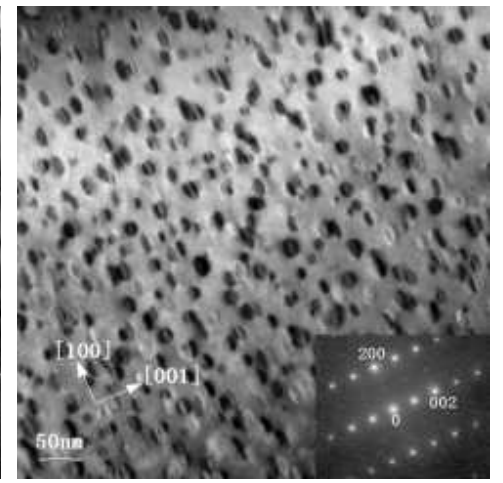

(c) $4 \mathrm{~h},[010]$

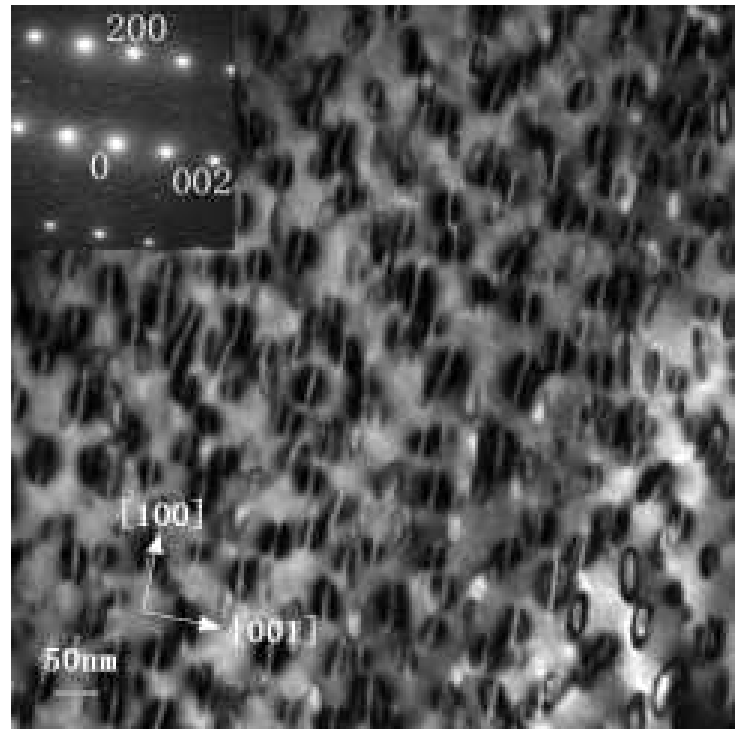

(d) $17 \mathrm{~h},[010]$

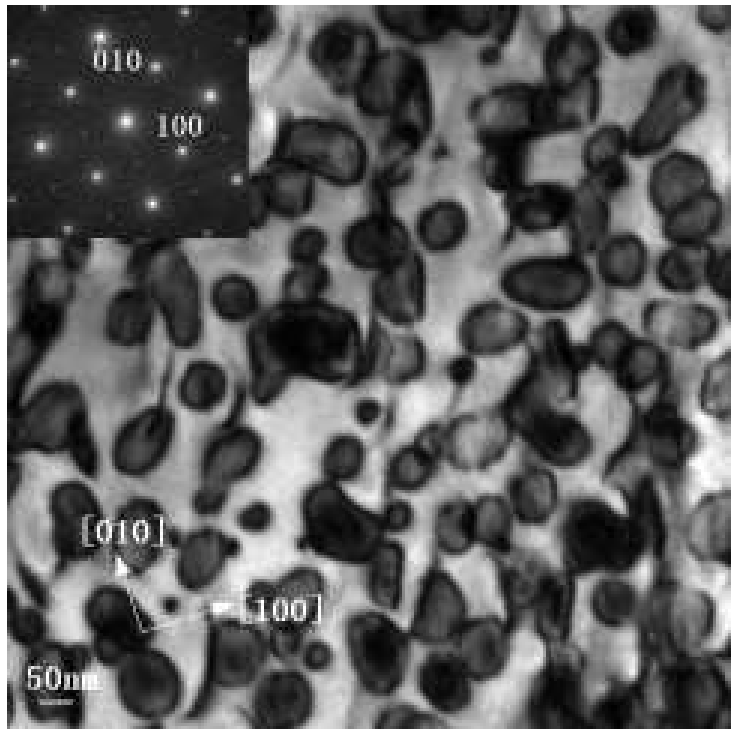

(e) $65 \mathrm{~h},[001]$

Abbildung 6.3: TEM-Dunkelfeld-Aufnahmen von $\mathrm{Ti}_{46} A l_{52} A g_{2}$ nach verschiedenen Auslagerungszeiten mit den selektierten Elektronenstrahlrichtungen der $\gamma$-Phase. In (a), der nicht wärmebehandelten Probe ist die $\gamma$-Phase dominant. Mit wachsender Auslagerungszeit bilden sich $\mathrm{L}_{2}$-Ausscheidungen. Die eingefügten SADP-Beugungsbilder (selected area diffraction pattern) zeigen die Orientierung. Zudem wurden sie zum Bestimmen der vorliegenden Phasen genutzt. Die hellen Punkte stammen von der $\gamma$-Matrix (L10), die sehr schwachen Punkte dazwischen von den L1 $1_{2}$-Ausscheidungen, die dafür sorgen, dass nicht nur für (001) sondern auch für (010) und (100) Überstrukturreflexe auftauchen. In $(b, c, d)$ lässt sich in den $\mathrm{L}_{2}$-Ausscheidungen deutlich eine Linie in [100]-Richtung sehen. Diese ist in der Dehnung der Ausscheidungen begründet, die sich der $\gamma$-Matrix anpassen. Dieser Effekt ist bei den großen Ausscheidungen (e) nicht mehr zu sehen. 


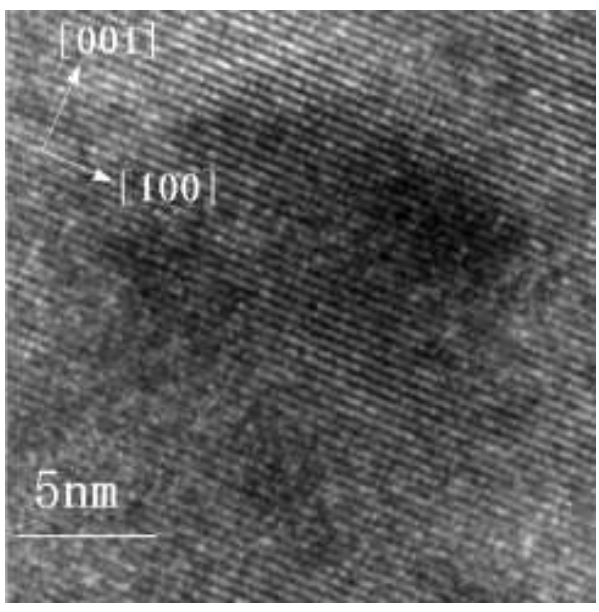

(a) $1 \mathrm{~h},[010$

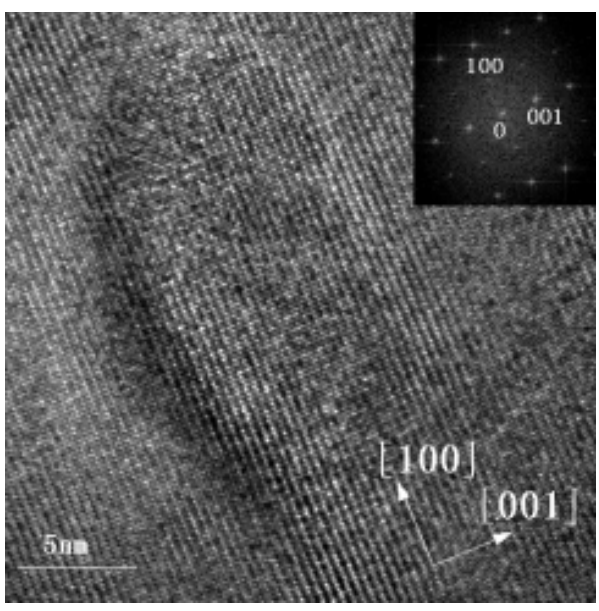

(c) $4 \mathrm{~h},[010]$

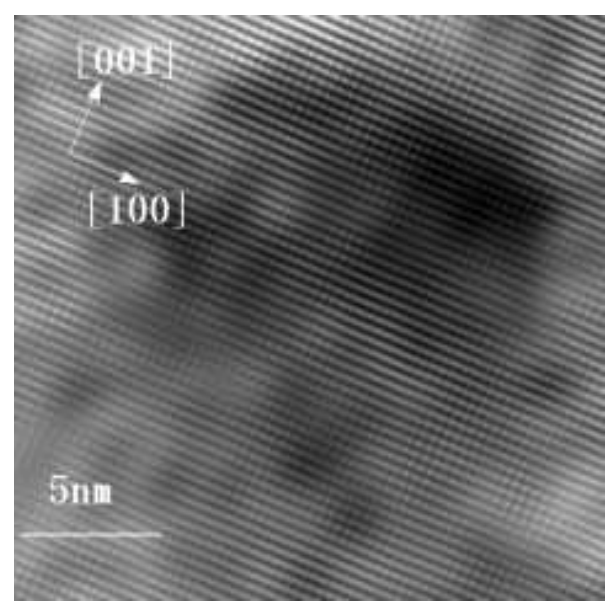

(b) 1h, [010], IFFT

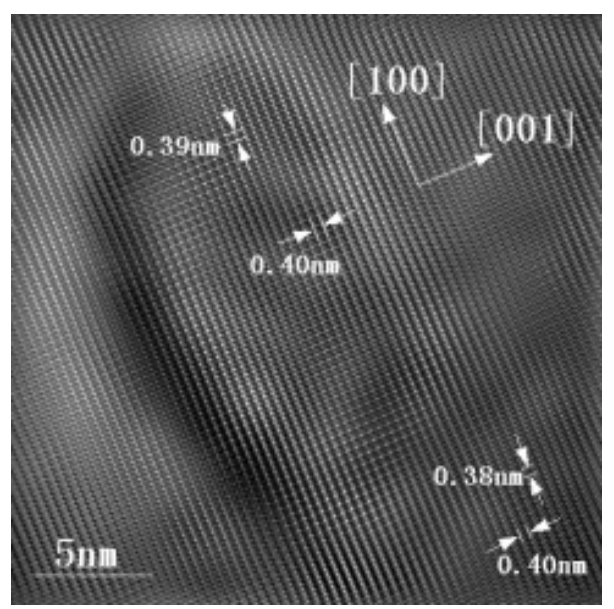

(d) 4h, [010], IFFT

Abbildung 6.4: HRTEM-Aufnahmen von $\mathrm{Ti}_{46} \mathrm{Al}_{52} \mathrm{Ag} \mathrm{g}_{2}$. Nach einer Auslagerungszeit von $4 \mathrm{~h}$ weicht die Gitterkonstante der $L 1_{2}$-Ausscheidungen von der der L1 $1_{0}$-Matrix in [100]Richtung ab, während dies in [001]-Richtung noch nicht der Fall ist. 


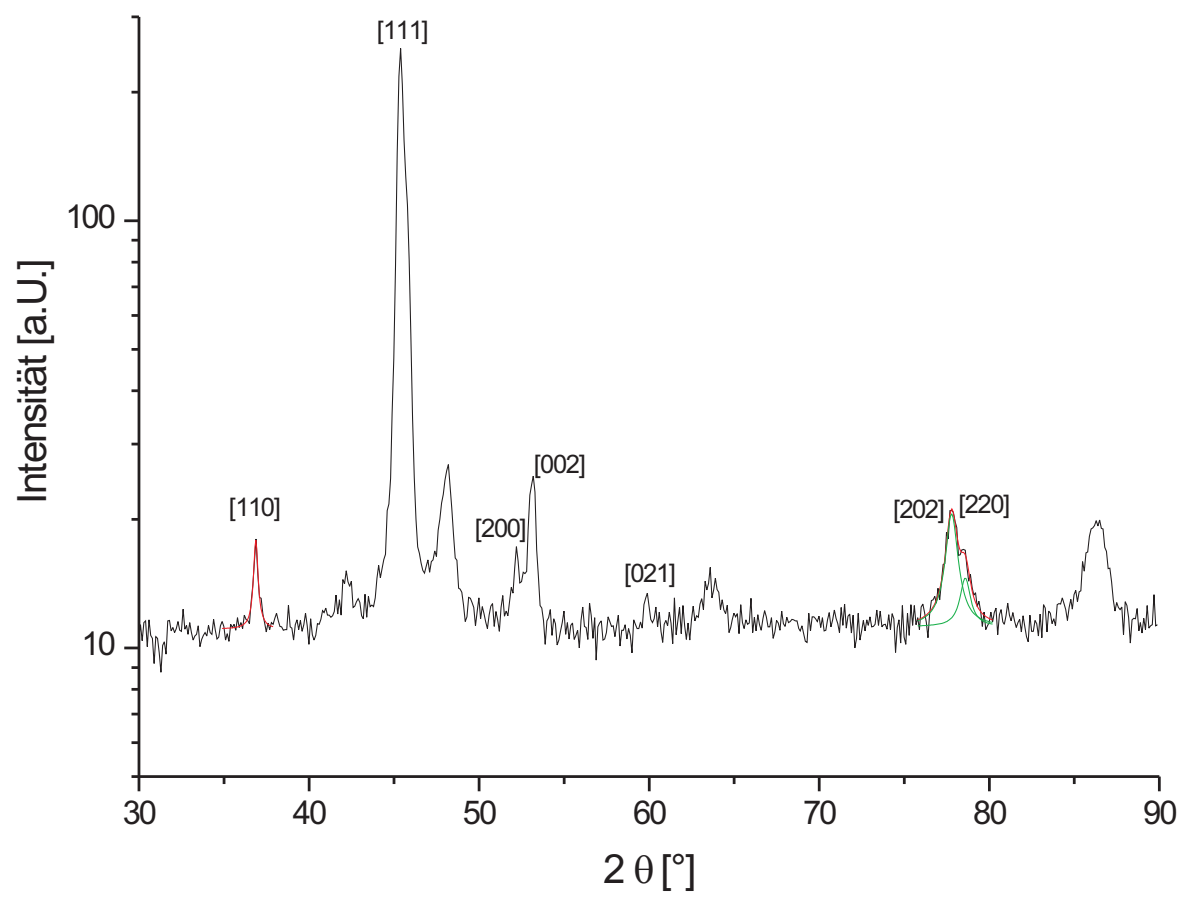

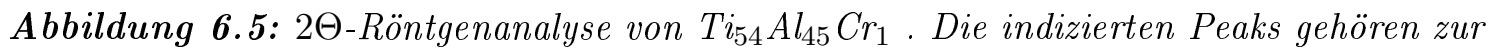
$\gamma$-Phase. Man beachte die Aufspaltung der Peaks. Die farbig markierten Peaks wurden mit der unter ihnen liegenden Fläche (abzüglich des Hintergrundsignals) zur Berechnung des Ordnungsparameters herangezogen. Die nicht indizierten Peaks sind vermutlich durch die $\alpha_{2}$-Phase bedingt. 
Sein Lösungsvorschlag, die Ordnung über den Abstand des [020], [200]-Peaks zum [002]-Peak abzuschätzen, ist in unserem Fall leider ebenfalls nicht möglich, da die Gitterverzerrung durch die ternären Legierungszusätze ebenfalls Einfluss auf diesen Abstand hat. Es wird also hier dennoch klassisch aus den theoretisch zu erwartenden, mit CarineCrystal 3.1 ermittelten, Werten für die Intensitäten $I_{\text {theo }}$ im Vergleich mit den real vorliegenden Intensitäten der Ordnungsgrad bestimmt. Wobei die Formel [Haas1994] leicht anders aussieht als gewohnt, da sich die Peaks aufsplitten. Die Intensitäten in den geschweiften Klammern beziehen sich deshalb immer auf die Summe der Intensitäten von 2 Peaks. Deswegen sollte das Ergebnis für den Ordnungsparameter ${ }^{1}$

$$
s=\frac{I_{[110]}}{I_{\text {theo },[110]}} \frac{I_{\text {theo },\{220\}}}{I_{\{220\}}}=0.87(7)
$$

im Falle von $\mathrm{Ti}_{54} \mathrm{Al}_{45} \mathrm{Cr}_{1}$ (ohne Wärmebehandlung) lediglich als Beleg gewertet werden, dass tatsächlich eine gute Ordnung vorliegt. Wie gut diese tatsächlich ist, lässt sich allerdings nicht anhand der Messung herausfinden, da nicht bekannt ist, wie sich die vorliegende $\gamma$-Phase genau zusammensetzt. Bei der vorliegenden nominellen Zusammensetzung ist es durchaus sinnvoll anzunehmen, dass die Zusammensetzung der Phase am Grenzbereich ihrer Existenz liegt. Somit kann man von nahezu perfekter Ordnung sprechen. Da außer der Ag-haltigen Probe alle anderen ebenfalls eine lamellare Struktur vorweisen, wurden für diese keine weiteren Analysen gemacht. Von der Ag-haltigen Phase stand leider nicht genug Material zur Verfügung um in vertretbarer Zeit Röntgenaufnahmen zu erstellen.

Ein weiterer interessanter Punkt bei der Betrachtung des Spektrums ist, dass die aufgesplitteten Peaks nicht in den Intensitäten auftauchen wie man sie erwarten würde. Die zur Überstrukturrichtung [002] und [220] gehörenden Peaks, die aus der Aufsplittung des Peaks der nicht geordneten Phase hervorgehen, sind viel zu hoch. Dies ist auch der Grund, warum die Normierung der gesplitteten Peaks in der verwendeten Formel nicht getrennt sondern gemeinsam erfolgt. Die Ursache für dieses Phänomen sind möglicherweise die häufig vorkommenden [011]-Zwillinge. Es ist durchaus sinnvoll anzunehmen, dass Überstruktur-Zwillinge gegenüber anderen Zwillingen energetisch günstiger sind und dadurch vermehrt auftreten. Dadurch kann sich eine Vorzugsrichtung der $\gamma$-Lamellen in einem Korn einstellen ${ }^{2}$, bei der die Überstrukturrichtung eher parallel als senkrecht zur Lamellenausrichtung vorliegt. Mit Hilfe der Bragg-Gleichung lassen sich aus den Positionen der aufgesplitteten

\footnotetext{
${ }^{1}$ Der Fehler wurde anhand von Gaussfits abgeschätzt

${ }^{2}$ Global gibt es keine Vorzugsorientierung
} 
Peaks die Abmessungen der Einheitszelle errechnen. Das Verhältnis der Abmessung in [001]-Richtung im Vergleich zu einer der anderen Richtungen ist 1.02, wie nach [Meno1996] erwartet wird.

\subsection{2 $\mathrm{Cu}_{3} \mathrm{Au}$}

Röntgenanalysen von $\mathrm{Cu}_{3} \mathrm{Au}$ wurden häufig durchgeführt, da es als Referenzsystem gilt [Star2007]. Deswegen war eigentlich ein guter Ordnungsgrad zu erwarten. Dennoch wurde auch eine Röntgenmessung durchgeführt, die aufgrund der geringen Materialmenge, die dafür zur Verfügung stand, leider einen recht hohen Fehler aufweist und einen Ordungsgrad $s=0.48(5)$ ergibt. Dies ist zunächst einmal überraschend gering, zumal die Probe über einen sehr langen Zeitraum ausgelagert wurde. Der durchschnittliche Korndurchmesser $D$ lässt sich nach der Scherrer-Formel (Gl. 6.1) für kugelförmige Körner aus der Halbwertsbreite $B$ des Gaussfits des rechten, nicht zur Überstrukturrichtung gehörenden, Teils des aufgesplitteten \{220\}-Peaks beim Winkel $2 \Theta=85.00(1)$ ! berechnen.

$$
D=\frac{0.9 \lambda}{B \cos \Theta}
$$

Die Annahme kugelförmiger Körner ist in diesem Fall, im Gegensatz zu $\gamma$-TiAl, eine vertretbare Näherung. Die $\alpha$-Wellenlänge der Co-Anode ist $\lambda=0.179 \mathrm{~nm}$. Die Korngröße von (790 \pm 22$) \mathrm{nm}$ ist angesichts der langen Auslagerungszeit im erwarteten Bereich.

Der durchschnittlichen Domänendurchmesser von (49.8 \pm 1.2$)$ nm berechnet sich unter Verwendung der selben Formel beim Überstrukturpeak der [110]-Richtung bei $2 \Theta=39.568(3)^{\circ}$. Dieser Durchmesser ist in einem Bereich, in dem man einige Domänen innerhalb einer für das FIM präparierten Spitze erhält. Die geringe Domänengröße erklärt auch den geringen Ordnungsgrad. Da mit X-Ray immer über mehrere Domänen in einem Korn gemessen wird, wird ein Teil des Signals durch eine ungünstige Lage der anderen Domänen wieder ausgelöscht. Innerhalb einer Domäne dürfte der Ordnungsgrad erheblich höher liegen. Zudem kann nicht ausgeschlossen werden, dass außerhalb der im TAP gemessenen Körner, die Zusammensetzung der Probe stark vom nominellen Wert abweicht. Dies würde ebenfalls als geringerer Ordnungsgrad detektiert werden. 


\section{$6.3 \quad$ FIM}

FIM-Aufnahmen dienten im Rahmen dieser Arbeit vor allem der Identifikation der kristallographischen Richtung, in die gemessen wurde. Diese Aufnahmen sind deshalb im Abschnitt 6.4 bei den zugehörigen TAP-Messungen zu finden. In diesem Abschnitt zur Charakterisierung der Mikrostruktur werden nur einige Aufnahmen präsentiert. Als Bildgas wurde dabei, im Falle von Wolfram, Helium bei $5 \cdot 10^{-5}$ mbar und für alle anderen Materialien Neon bei Drücken im Bereich $10^{-5}$ bis $10^{-4}$ mbar verwendet.

Die Indizierung von FIM-Bildern erfolgt dabei primär aufgrund geometrischer Überlegungen. Einerseits muss die Anordnung der Pole auf dem FIM-Bild stimmen, andererseits kann man aus den zu erwartenden Ebenenabständen die Prominenz der Pole herleiten [Mill1996]. Prominenz bedeutet in diesem Fall nicht die Helligkeit, sondern die Sichtbarkeit der Ringe im FIM-Bild. Diese wird umso besser sein, je größer die Ebenenabstände sind. Für ein kubisch flächenzentriertes (kfz) Gitter ergibt sich somit eine Reihenfolge der Prominenz der Pole von (111), (200), (220), (113), (240), (224) sowie für ein kubisch raumzentriertes (krz) Gitter von (110), (200), (112), (130), (222), (231). Für geordnete Legierungen ändert sich diese Reihenfolge, da aufgrund der unterschiedlichen Feldverdampfungsfeldstärken $E_{\text {Evap }}$, die ein schnelles Verdampfen einer Schicht mit niedrigem $E_{\text {Evap }}$ unter einer Schicht mit hohem $E_{\text {Evap }}$ bewirken, die Kanten von Polen in Überstrukturrichtungenen steiler werden. Für die $\mathrm{L} 1_{0}$-Struktur ergibt sich damit eine Reihenfolge der Prominenz von (001), (110), (111), (200), (120), (220), (112), (113), (240). Für die L1 $2_{2}$-Struktur erhält man eine Reihenfolge von (100), (110), (111), (120), (112), (113), wobei einige Richtungen nur noch geordnet auftauchen. Die Prominenz ist nicht gleichbedeutend mit der Helligkeit der Pole sondern eher mit dem Kontrast der Ringe gegenüber der Umgebung 3 . Die Helligkeit hängt jeweils davon ab, wie weit ein Objekt aus der sonstigen Spitze hervorragt. Es ragt umso weiter hervor, je höher $E_{\text {Evap }}$ dort ist. Die Helligkeit kann also als Indiz für die Feldverdampfungsfeldstärke gesehen werden.

\footnotetext{
${ }^{3}$ [Mill1996] behauptet zwar, dass die prominenten Pole herausragen, jedoch sind sie auf den FIMBildern klar dunkler. Lediglich die Ringe sind kontrastreicher, da sie vor dunklerem Hintergrund stehen.
} 


\subsubsection{TiAl}

Ein naheliegender Einfluss höherer Dotierungen des ternären Elements ist eine weniger geordnete Feldverdampfung, was sich in einer raueren Spitzenoberfläche äußert. Bereits in [Boll2005] wurde dies festgestellt. In Abb. 6.6 ist dargestellt, wie sich das FIM-Bild mit wachsender Niob-Konzentration verändert. Die vielen hellen Punkte können dabei aufgrund ihres wachsenden Anteils Niob zugeordnet werden. Zudem wird für $\mathrm{Nb}$ auch ein weit höheres $E_{\text {Evap }}$ von $37 \mathrm{~V} / \mathrm{nm}\left(\right.$ für $\mathrm{Nb}^{2+}$ ) im Vergleich zu $26 \mathrm{~V} / \mathrm{nm}$ bei $\mathrm{Ti}^{2+}$ und $33 \mathrm{~V} / \mathrm{nm}$ für zweifach geladenes $\mathrm{Al}\left(\mathrm{Al}^{+}=19 \mathrm{~V} / \mathrm{nm}\right)$ erwartet [Mil11996]. Nb Atome sollten also exponiert auftauchen und somit Stellen markieren an denen vermehrt Bildgasatome ionisieren.

Die Prominenz der Pole folgt weitgehend den Erwartungen, lediglich die (200)-Pole sind weit besser sichtbar als die (111)-Pole. Dies zeigt, dass eine einfache geometrische Überlegung die Sichtbarkeit der Pole nicht richtig vorhersagt. Die Abdunkelung der (111)-Pole liegt an der dort verringerten Feldverdampfungsfeldstärke, die diese Stellen im Vergleich zum Rest der Oberfläche absenkt.

Nimmt man an, dass die hellen Punkte Niob zugeordnet werden können, so kann man durch die Beobachtung der Feldverdampfung an einem geordneten Pol eine bevorzugte Platzbesetzung beobachten. Ebenen mit hoher Feldverdampfungsfeldstärker bilden heller ab als Ebene mit niedriger Feldverdampfungsfeldstärke. An dieser Stelle ist es noch nicht möglich, zu entscheiden, welche Ebene welchem Element (Ti oder $\mathrm{Al}$ ) zugeordnet werden muss. Es wird sich später (Kap.6.8.1.1) zeigen, dass $\mathrm{Al}$ die höhere Feldverdampfungsfeldstärke besitzt. Mit diesem Wissen lässt sich durch Beobachtung der Feldverdampfung in Abb. 6.6 ein Platzbesetzung des Nb von Ti-Plätzen feststellen.

Im FIM-Bild von $\mathrm{Ti}_{46} \mathrm{Al}_{52} \mathrm{Ag}_{2}$ in Abb. 6.8(a) lässt sich nichts auffälliges feststellen. Die Position des Silbers kann nicht festgestellt werden, da es mit $E_{\text {Evasp }}=24 \mathrm{~V} / \mathrm{nm}$ keine exponierten Stellen besetzt. Zudem ist die Austrittsarbeit für Elektronen mit $4.6 \mathrm{eV}$ im Vergleich zu 4.0 bis $4.1 \mathrm{eV}$ von Ti,Al,Nb wesentlich höher und lässt ebenfalls ein dunkleres Abbildungsverhalten erwarten. Die verringerte Ag-Konzentration in den TAP-Messungen (Kap. 6.10.2) lässt erwarten, dass sich Ag-reichere Cluster bilden. Auch solche konnten allerdings nicht beobachtet werden.

Für $\mathrm{Ti}_{52} \mathrm{Al}_{45} \mathrm{Cr}_{3}$ in Abb. 6.8(b) lässt sich ebenfalls aus dem FIM-Bild nichts über die Position von Chrom aussagen. Analog zu Ag ist hier die Feldverdampfungsfeldstärke 


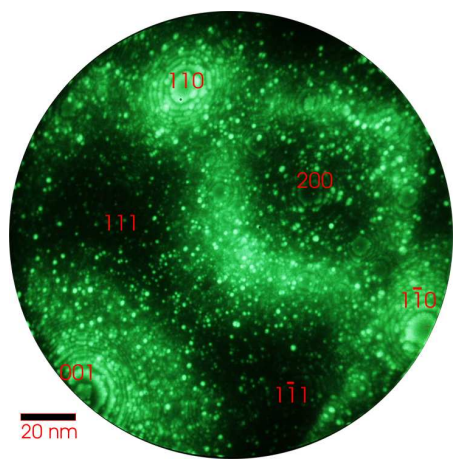

(a) $\mathrm{Ti}_{51} \mathrm{Al}_{48} \mathrm{Nb}_{1}$

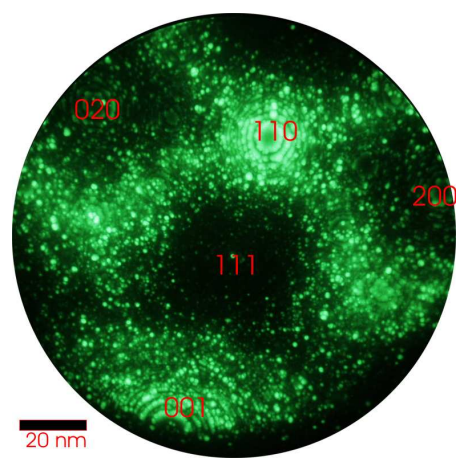

(b) $\mathrm{Ti}_{47} \mathrm{Al}_{48} \mathrm{Nb}_{5}$

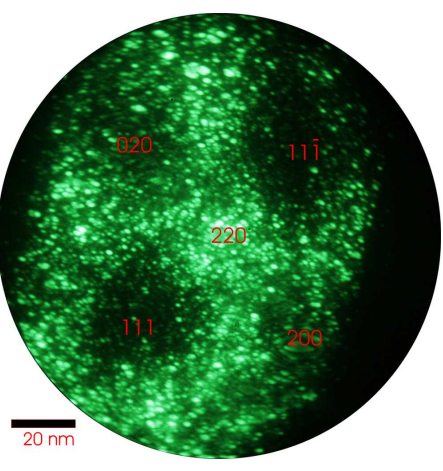

(c) $\mathrm{Ti}_{42} \mathrm{Al}_{48} \mathrm{Nb}_{10}$

Abbildung 6.6: FIM-Aufnahmen bei T=65K mit Proben, die 44h@1273 K ausgelagert wurden. Mit von (a) nach (c) wachsender Niob-Konzentration lassen sich immer weniger Polringe unterscheiden. Es wird zudem ein deutlich höherer Anteil an hellen Punkten feststellbar, die deswegen mit $\mathrm{Nb}$ assoziiert werden können.
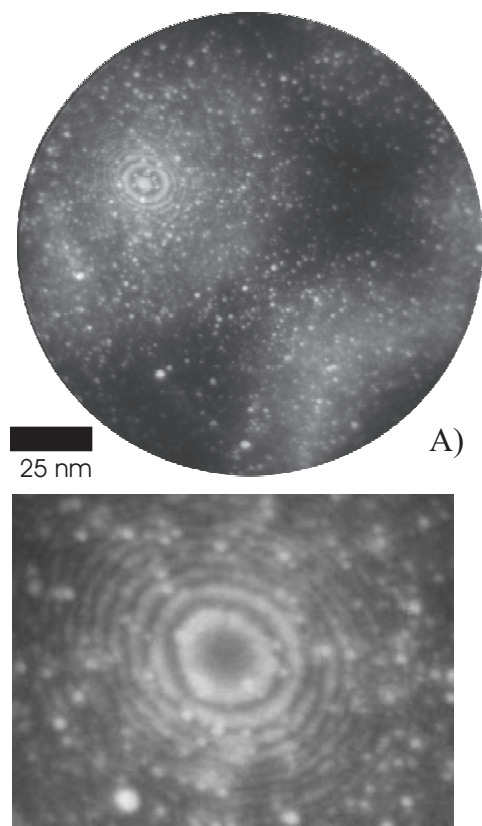

D)

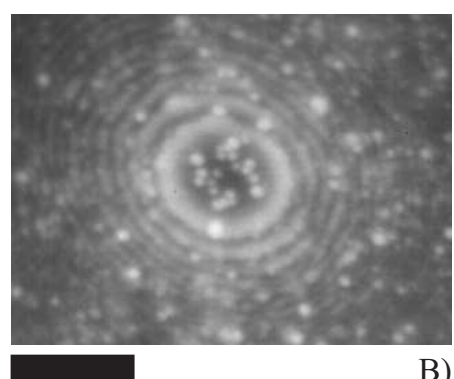

B)

$$
10 \mathrm{~nm}
$$

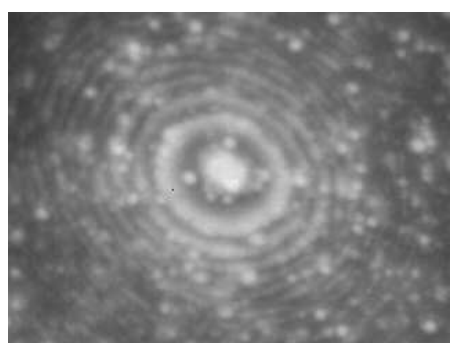

E)

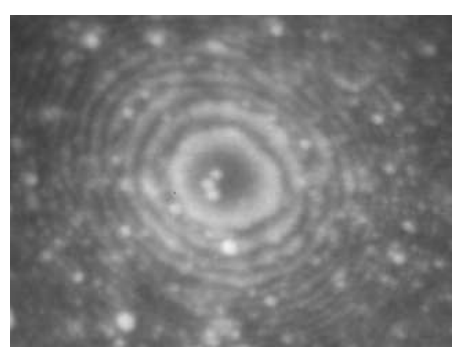

C)

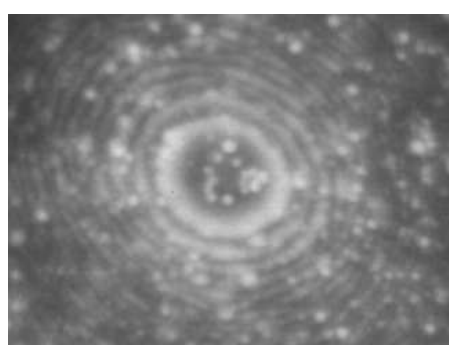

F)

Abbildung 6.7: FIM-Verdampfungssequenz einer $\gamma-T i_{51} A l_{48} N b_{1}$-Spitze bei $60 \mathrm{~K}$ und nahezu konstanter Spannung von $13.4 \mathrm{kV}$. In A) ist die gesamte Spitze mit dem Rest einer Al-Schicht auf dem (001)-Pol oben links zu erkennen. B) zeigte eine Vergrößerung des (001)-Pols. Die Al-Schicht ist vollständig verdampft und die darunter liegenden hellen NbAtome der $\mathrm{Nb} / \mathrm{Ti}$-Ebene werden sichtbar. Weitere Feldverdampfung in C) lässt in D) die nachfolgende Al-Schicht nach Entfernen des letzten Nb-Atoms erkennen. In E) ist der letzte Rest der Al-Schicht zu sehen, der danach die nächste $\mathrm{Nb} / \mathrm{Ti}$-Schicht in F) freilegt. Übernommen aus [Boll2005].

mit $29 \mathrm{~V} / \mathrm{nm}$ für $\mathrm{Cr}^{2+}$ bzw. $27 \mathrm{~V} / \mathrm{nm}$ für $\mathrm{Cr}^{+}$im Bereich von der von Ti und $\mathrm{Al}$. Zudem ist die Austrittsarbeit mit $4.6 \mathrm{eV}$ wiederum höher und lässt somit ebenfalls eher eine dunkle Abbildung erwarten. 


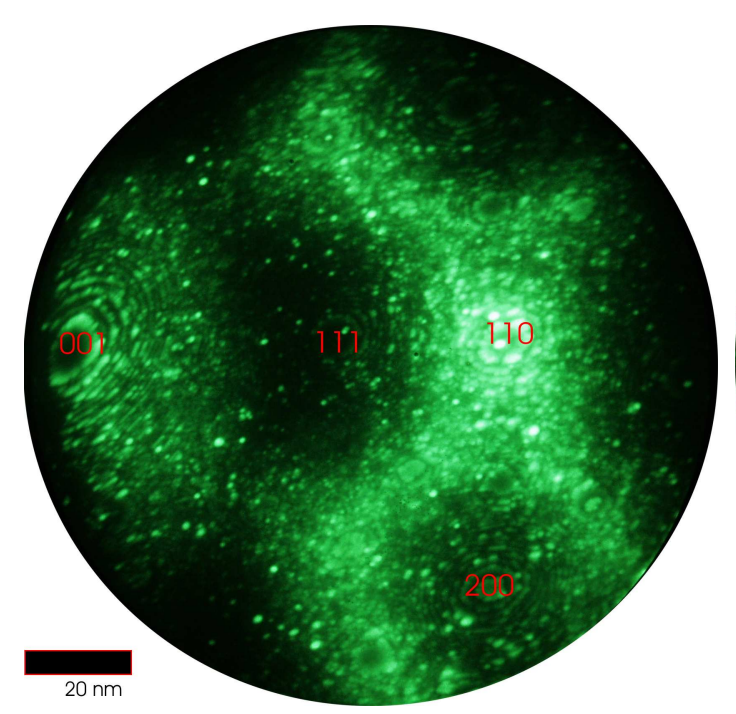

(a) $\mathrm{Ti}_{46} \mathrm{Al}_{52} \mathrm{Ag}_{2}$

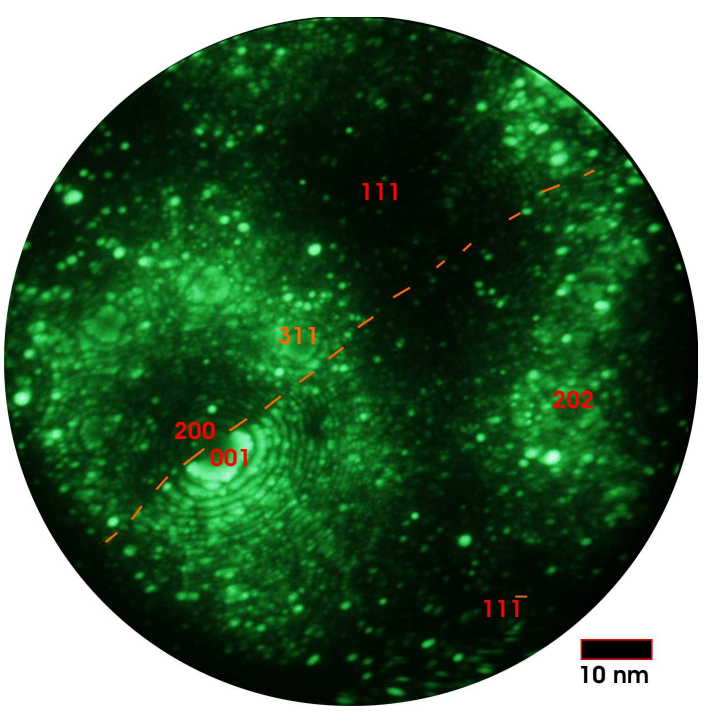

(b) $\mathrm{Ti}_{52} \mathrm{Al}_{45} \mathrm{Cr}_{3}$ mit Antiphasengrenze

Abbildung 6.8: $\mathrm{Ti}_{46} A l_{52} A g_{2}$ bei $40 \mathrm{~K}$. Die hellen Punkte sollten hier lieber nicht mit $\mathrm{Ag}$ identifiziert werden, da dies mit dem erwarteten Abbildungsverhalten nicht übereinstimmt. Selbiges gilt für $\mathrm{Cr}$ in (b). Hier sieht man sehr gut eine $\gamma / \gamma$-Antiphasengrenze an einem (001)/(200)-Pol.

In Abb. 6.9 ist eine Cr-haltige Probe abgebildet. Es liegt offenbar eine $\gamma / \alpha_{2} / \gamma$ Phasenabfolge vor. Die lamellare Struktur kann gut erkannt werden. Die Polringe in der $\gamma$-Phase sind weit besser zu erkennen als in der $\alpha_{2}$-Phase. Dies erklärt sich durch den breiteren Existenzbereich dieser Phase, der größere Fehlbesetzungsanteile erlaubt als die $\gamma$-Phase. Dieser Effekt ist vergleichbar mit dem Einfluss der steigenden Nb-Konzentration in Abb. 6.6. Auch $\gamma / \gamma$-Grenzen lassen sich, wie in Abb. 6.8(b) an einem (001)/(200)-Pol besonders deutlich wird, häufig beobachten.

\subsection{2 $\mathrm{Cu}_{3} \mathrm{Au}$}

In Abb. 6.10 ist das FIM-Bild einer $\mathrm{Cu}_{3} \mathrm{Au}$-Probe zu sehen. Es befinden sich mehrere Domänen in dem Korn, die mit dem durch die Röntgenmessung bestimmten Domänendurchmesser (Kap. 6.2.2) von (49.8 \pm 1.2$)$ nm gut vereinbart sind. Die Orientierung lässt sich bestimmen, wenn man davon ausgeht, dass die Domänen sich alle aus demselben Korn entwickelt haben. In dem Fall stellt man eine gute Übereinstimmung des FIM-Bildes mit den erwarteten Positionen einer monokristallinen Probe fest. Die Bestimmung der prominenten Pole als geordnete und der dunkleren als nicht geordnete ergibt sich aus der erwarteten höheren Feldverdampfungsfeldstärke der geordneten Pole. Zudem liegen an den geordneten Polen Doppelstufen 


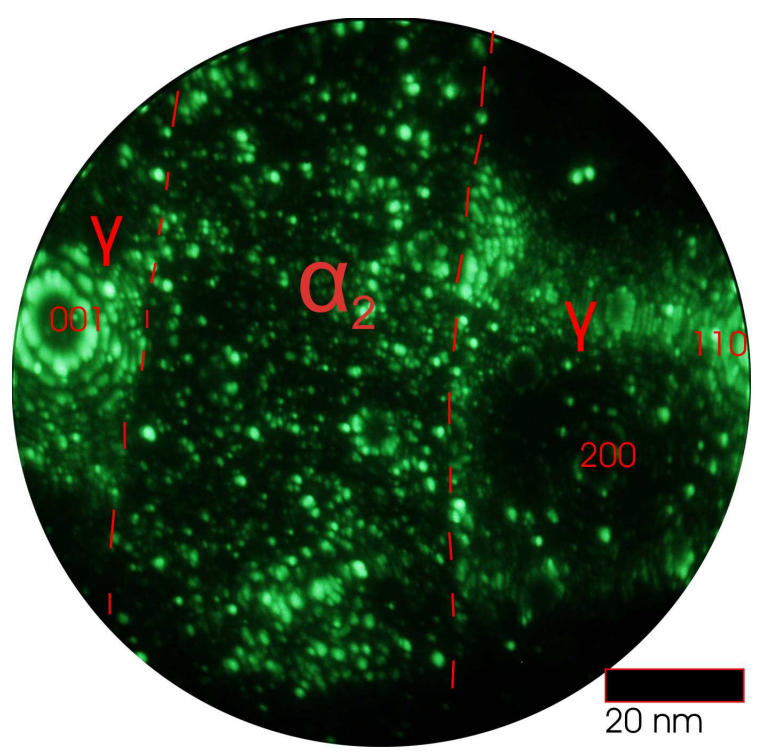

Abbildung 6.9: $\mathrm{Ti}_{52} \mathrm{Al}_{45} \mathrm{Cr}_{3}$ bei $40 \mathrm{~K}$. Eine $\gamma / \alpha_{2} / \gamma$-Lamellenabfolge kann beobachtet werden

über eine reine $\mathrm{Cu}$ und eine gemischte $\mathrm{Cu} / \mathrm{Au}$-Ebene vor, die für die gut sichtbaren Ringe dieser Richtungen sorgen. Aufgrund der Doppelstufe wurden die Pole (in Anlehnung an die aufgehobene Auslöschungsregel) als \{110\}-Pole bezeichnet.

\subsection{TAP: Feldverdampfungsfeldstärken}

\subsubsection{Messparameter}

Die TAP-Messparameter für TiAl-Proben wurden bereits in [Boll2005] variiert. Generell ist es vorteilhaft bei möglichst tiefen Temperaturen zu arbeiten, da sich dort die $E_{\text {Evap }}$ der unterschiedlichen Komponenten annähern. Eine möglichst hohe Pulsspannung $U_{P u l s}$ ist ebenfalls geeignet um Unterschiede in der Feldverdampfung der Komponenten auszugleichen. Als optimaler Kompromiss zwischen Abrisswahrscheinlichkeit und guten Messergebnissen sind als Messtemperatur durchgängig $40 \mathrm{~K}$ und als Pulsspannungsanteil $U_{\text {Puls }}=22.5 \%$ der Basisspannung $U_{\text {Basis }}$ verwendet worden. Die $\mathrm{Cu}_{3} \mathrm{Au}$-Proben wurden ebenfalls bei $40 \mathrm{~K}$ gemessen. Allerdings bei einem Pulsspannungsanteil von $U_{P u l s}=0.25 U_{\text {Basis }}$. Bei den Wolframspitzen wurden ebenfalls 40 $\mathrm{K}$ als Temperatur eingestellt. Die Pulsspannung betrug hier $20 \%$ von $U_{\text {Basis }}$. Für andere Materialien wurden $U_{\text {Puls }}=0.15 U_{\text {Basis }}$ bei $40 \mathrm{~K}$ verwendet. Die Spannung an den Kanalplatten wurde für jede Messung separat optimiert, um möglichst viele 


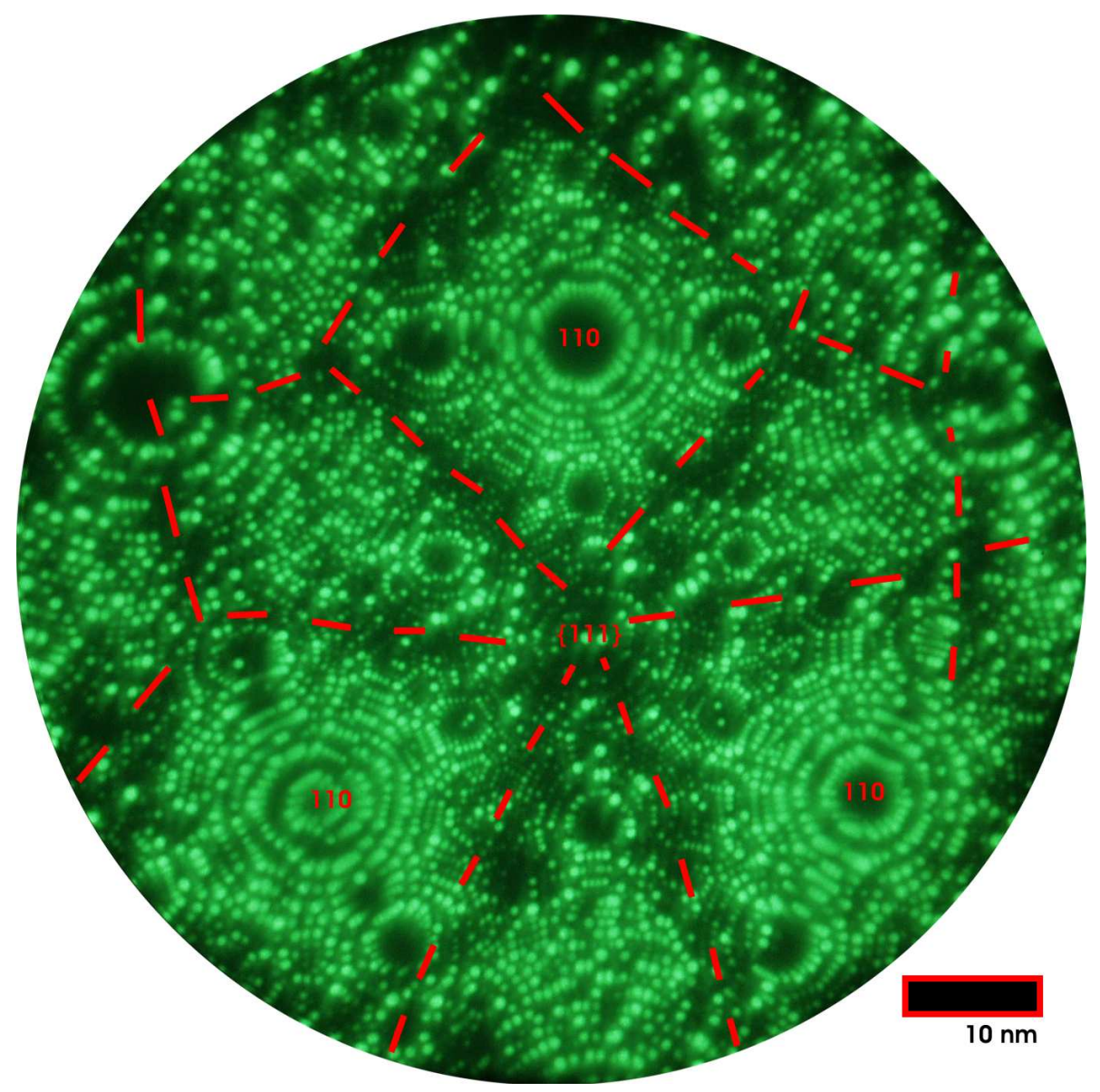

Abbildung 6.10: $C u_{3} A u$-Probe bei $7.4 \mathrm{kV}, 30 \mathrm{~K}, p_{\text {Neon }}=10^{-4}$ mbar: Man erkennt deutlich Domänengrenzen. Die drei hell leuchtenden um den Mittelpunkt angeordneten Pole sind geordnete (110)-Pole (abwechselnd eine reine Cu-Ebene mit einer gemischten CuAuEbene). In der Mitte befindet sich ein $\{111\}$-Pol, in dem mehrere Domänen aufeinandertreffen. In dem linken unteren (110)-Pol befindet sich zudem eine gut sichtbare Versetzung. Die Indizierung ist für alle Pole gleich um anzudeuten, dass es verschiedene Domänen sind. Deren Abgrenzungen sind (in etwa) durch die gestrichelten Linien gekennzeichnet. Wobei einige dieser Grenzen im Randbereich auch Kleinwinkelkorngrenzen oder Versetzungen sein könnten. 
Messereignisse mit 3-5 Anoden zu erreichen. Diese lagen bei $700 \mathrm{~V}$ und bei $\sim 2575$ $\mathrm{V}$ und wurden um bis zu $3 \mathrm{~V}$ variiert.

Für die LEAP-Messungen wurden auf den TAP-Erfahrungen basierte Parameter gewählt.

\subsubsection{Einstellen der Parameter der Rekonstruktion}

Der Parameter, der immer korrigiert werden muss, ist der Abstand zwischen Spitze und Detektor $L$, der so verändert wird, dass das Massenspektrum Peaks an den erwarteten Stellen zeigt. Der andere wichtige Parameter ist $E_{\text {Evap }} \beta$, der sich aus der Feldverdampfungsfeldstärke und einem Spitzengeometriefaktor $\beta$ zusammensetzt. Üblicherweise sollte man annehmen, dass die Feldverdampfungsfeldstärke sich bei identischen Messparametern nicht verändert und dieser Parameter somit vor allem durch $\beta$ bestimmt ist. Dies ist jedoch, wie sich im folgenden herausstellen wird, nicht korrekt. Die Einstellung erfolgt dadurch, dass der bekannte Ebenenabstand einer Richtung mit dem durch AtomVicinity-Dichteprofile ermittelten Ebenenabstand verglichen wird und $E_{\text {Evap }} \beta$ solange angepasst wird, bis beide übereinstimmen.

\subsubsection{Massenspektren}

\subsubsection{TiAl}

Betrachtet man Massenspektren der $\mathrm{TiAl}+\mathrm{X}-$ Proben, so stellt man fest, dass die Atome aller Elemente nahezu ausschließlich zweifach ionisiert auf dem Detektor auftreffen. Lediglich ein sehr kleiner Anteil Al und ein großer Teil des Ag sind einfach ionisiert. Dieses Verhalten bestätigt sich in allen Messungen, unabhängig von der kristallographischen Richtung. Es gibt für die Ag- und Nb-haltigen Proben auch keine Peaküberlappung. Bei den Cr-haltigen Messungen dagegen überlappen sich $\mathrm{Ti}_{50}^{2+}$ mit $\mathrm{Cr}_{50}^{2+}$ und $\mathrm{Al}_{27}^{+}$mit $\mathrm{Cr}_{54}^{2+}$. Die in diesen Peaks enthaltenen Cr-Atome sind nur $6.7 \%$ aller Cr-Atome und können deswegen ohne zu große Einbußen zusammen mit den entsprechenden $\mathrm{Al}$ und Ti-Atomen bei der Analyse ausgelassen werden. 


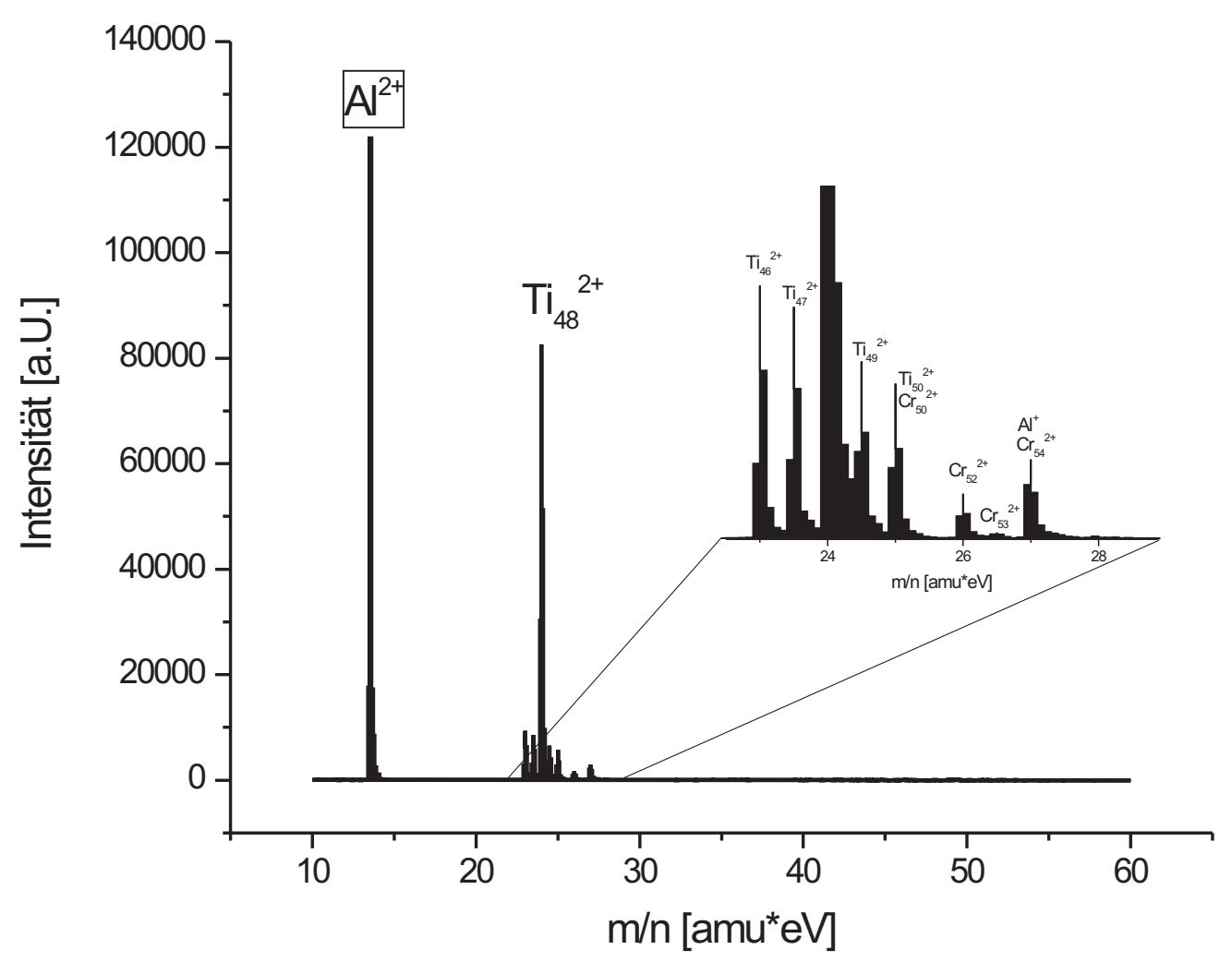

Abbildung 6.11: Massenspektrum von $\mathrm{Ti}_{52} \mathrm{Al}_{45} \mathrm{Cr}_{3}$. Es gibt Überschneidungen für $\mathrm{Cr}_{50}$ und $\mathrm{Cr}_{54}$. Diese Isotope haben einen Anteil von $4.3 \%$ bzw $2.4 \%$ von allen Cr-Isotopen. 


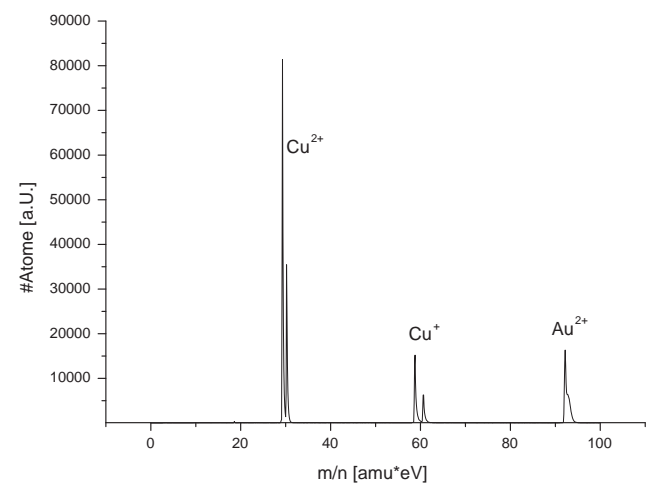

(a) bei (111)-Pol

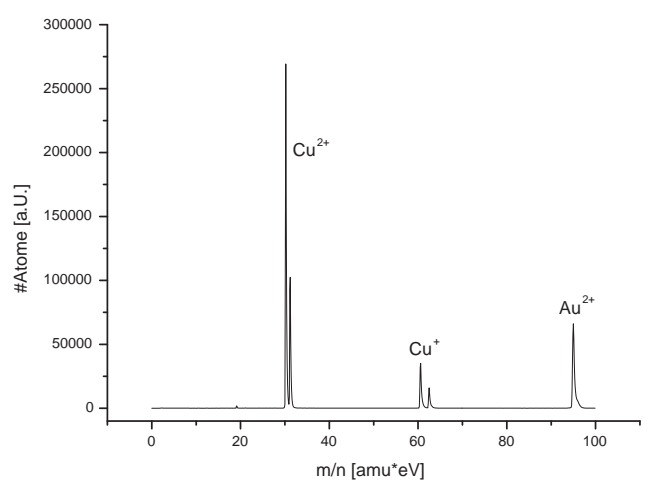

(b) bei (110)-Pol

Abbildung 6.12: Massenspektrum von $C u_{3} A u$ für verschieden Richtungen $(a, b)$. $A u^{+}$ taucht immer nur unwesentlich ( $<0.5 \%$ der Au-Atome) auf und ist deswegen nicht dargestellt. Cu besitzt 2 Isotope bei 62 und 65 amu. In der Nähe eines Überstrukturpols (b) findet man einen erhöhten Anteil von $\mathrm{Cu}^{2+}$.

\subsubsection{2 $\mathrm{Cu}_{3} \mathrm{Au}$}

Obwohl weniger Komponenten enthalten sind, weist das Massenspektrum von $\mathrm{Cu}_{3} \mathrm{Au}$ mehr Probleme auf. Gold wird zwar nur zweifach geladen detektiert, Kupfer jedoch tritt sowohl in einfach als auch zweifach geladen auf. Erwarten würde man bei beiden nach [Mil11996] einfach geladene Zustände, denn $E_{E v a p}\left(C u^{+}\right)=30 \mathrm{~V} / \mathrm{nm}$, $E_{\text {Evap }}\left(C u^{2+}\right)=43 \mathrm{~V} / \mathrm{nm}$ sowie $E_{\text {Evap }}\left(A u^{+}\right)=53 \mathrm{~V} / \mathrm{nm}, E_{\text {Evap }}\left(A u^{2+}\right)=54 \mathrm{~V} / \mathrm{nm}$. Da der Unterschied für Au zwischen beiden Ladungszuständen sehr gering ist, ist die Berechnung von $E_{\text {Evap }}$ ohnehin mit Vorsicht zu genießen. Der Anteil der einfach geladenen $\mathrm{Cu}$-Atome ist zudem für verschiedene kristallographische Richtungen unterschiedlich. Für die nichtgeordnete $<111>$-Richtung findet man überwiegend zweifach ionisierte Atome. Für die Superstrukturrichtungen hingegen ist der Anteil einfach geladener Ionen größer. Es ist allerdings nicht möglich, den Unterschied genau zu quantifizieren. Der Anteil der zweifach geladenen Ionen fällt mit dem Abstand zum Pol für geordnete Richtungen ab. Somit sind die die niedrig indizierten geordneten Pole für diesen Effekt, wie später in Abb.6.23 (Kap. 6.6) gezeigt wird, die alleinige Ursache.

\subsubsection{Bestimmung der Kristallstruktur}

Ein wesentlicher Vorteil des AtomVicinity-Algorithmus ist es, eine ausreichende Auflösung zu erhalten um Netzebenenabstände in laterale Richtungen zu bestimmen. 
Dies gelang in [Bol12005] leider nur mit recht enttäuschender Genauigkeit. Ein Grund war die damals unzureichende Programmierung des Algorithmus, die einerseits eine lange Rechenzeit verursachte, andererseits aber auch unbedingt große Speichermengen erforderte. Betriebssystembedingt ist es aber nicht möglich mit einem Programm mehr als 2 Gigabyte anzusprechen. In der aktuellen Version und angewandt auf neuere Messungen liefert der Algorithmus bessere Ergebnisse, da diese Speicherbeschränkung hier durch ein integriertes Verfahren zur Erstellung von 3D-Dichtekarten umgangen wird.

\subsubsection{W-Messung mit LEAP}

Im LEAP lässt sich ein Einfluss der Feldverdampfung in verschiedene kristallographische Richtungen feststellen. Vorteilhaft ist dabei, dass mehrere Pole innerhalb des Analysebereichs der Sonde positioniert werden können, wie es in Abb. 6.13(a) deutlich wird. Auch hier kann wieder AtomVicinity zur Auswertung verwendet werden. In diesem Fall sind tatsächlich einzelne Atome in $(x, y)$-Richtung unterscheidbar. Der Vorteil gegenüber der Göttinger Messung (Abschnitt 6.4.4.2) ist hierbei, dass nicht nur 2 (oder 3, die in einer Linie liegen) sondern mehrere kristallographische Richtungen zur Berechnung des Ergebnisses beitragen. Dadurch überschneiden sich im AtomVicinity mehrerer Ebenenstrukturen, die im Endergebnis erhöhte Dichten an den Atompositionen bewirken. Dies funktioniert vor allem deswegen gut, da die Spitze insgesamt ein ziemlich einheitliches Verdampfungsverhalten hat, wie in Tabelle 6.1 zu sehen ist. Die Ebenenabstände weichen kaum von den erwarteten Werten ab. Die Winkel $\varphi$ zwischen den Polen sind zumindest in eine Richtung auch zutreffend. Eine leichte Stauchung in die andere Richtung reicht lediglich für einen kleinen Effekt auf die Ebenenabstände $d$. Die theoretischen Ebenenabstände ergeben sich aus dem Gitterparameter $a=0.316 \mathrm{~nm}$ für Wolfram [Mill1996].

Ein verkleinerter Ebenenabstand bedeutet im Grunde, dass mit einer zu großen Feldverdampfungsfeldstärke $E_{\text {Evap }}$ im Rekonstruktionsalgorithmus gerechnet wurde. Dies bewirkt eine Überschätzung der lokalen Krümmung. In der Tat sieht man im FIM-Bild einer W-Spitze in Abb. 6.15, dass der Pol kaum sichtbar ist. Die anderen $\{123\}$-Pole sind sogar praktisch nicht zu erkennen. Die Atome verdampfen also zu großen Teilen, während die lokale Krümmung kleiner ist als an anderen Positionen der Spitze. Dies bedeutet, dass die Spitzenkrümmung an dieser Stelle geringer ist als im Rest der Spitze. Die Rekonstruktion geht demnach von einer falschen 


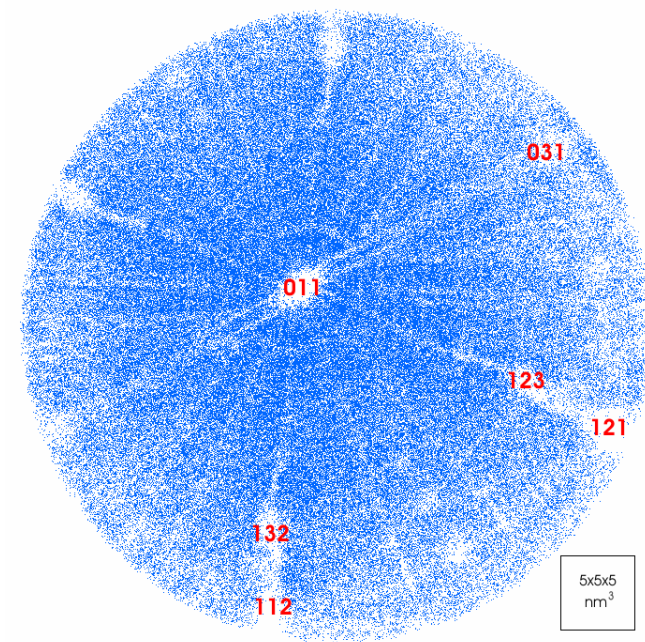

(a) Gesamte Messung: Aufsicht

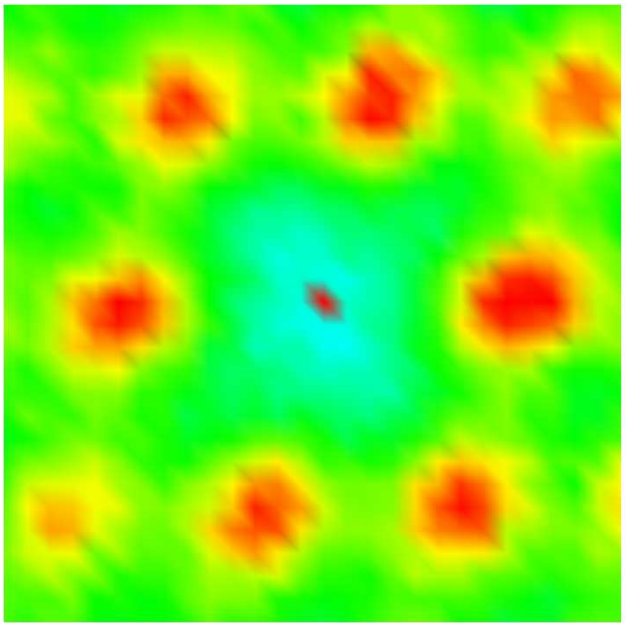

(c) AtomVicinity: Mittlere Ebene

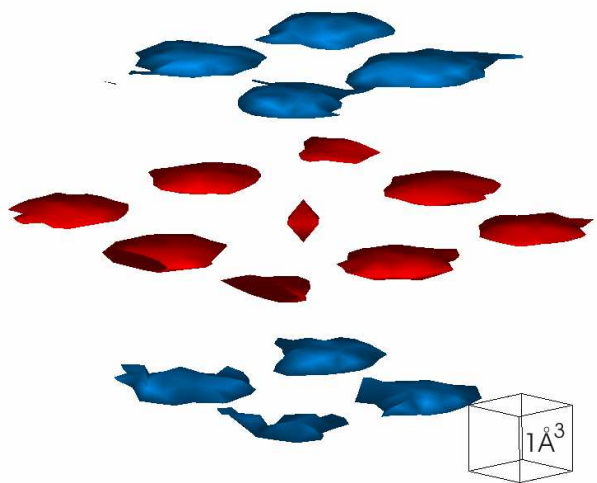

(b) AtomVicinity: Isosurfaces

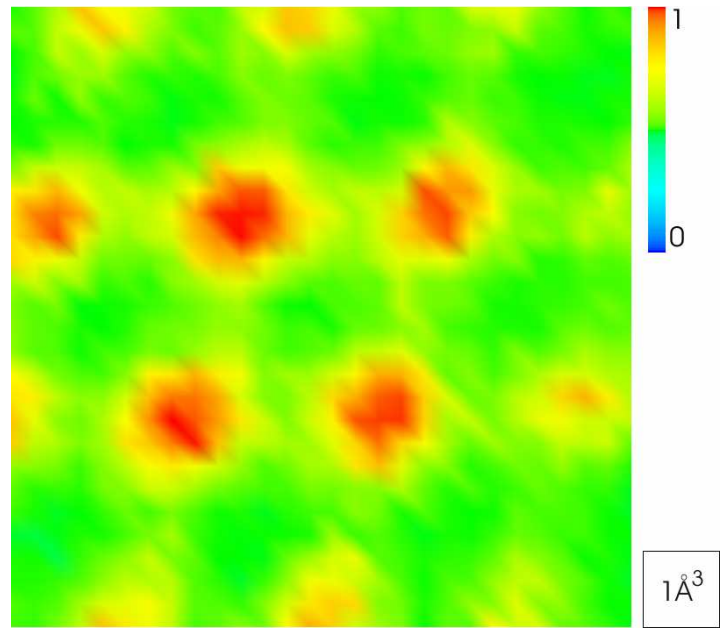

(d) AtomVicinity: Obere Ebene

Abbildung 6.13: Eine W-LEAP-Messung bei 43.8K, $U_{\text {Puls }}=0.15 U_{\text {Basis. }}$. Mehrere Pole ließen sich zuordnen (a). Darüber hinaus wurden die in Tabelle 6.1 Ebenenabstände und Winkel an den markierten Polen gemessen. AtomVicinty ausgeführt auf das gesamte Volumen ergibt das in $(b, c, d)$ dargestellt Ergebnis. In (b) sind die Atompositionen durch Isosurfaces für Dichten über 67\% der höchsten Dichte abseits des Zentrums sichtbar gemacht. Die mittlere Ebene mit $z \sim 0$ ist hierbei rot markiert. Die anderen beiden Ebenen sind blau. Randatome wurden in den oberen und unteren Ebenen herausgeschnitten. Beachtenswert ist hierbei, dass die Atompostion nun nicht nur in $z$-sondern auch in $(x, y)$-Richtung ermittelt werden kann. In (c) ist die Flächendichteverteilung der mittleren Ebene dargestellt. Die Atome sind hier tatsächlich in etwa an den Positionen, an denen sie erwartet werden. In (d) ist die Dichteverteilung in der oberen Ebene dargestellt, die in (c) durch die 4 oberen Atome gebildet wird. Auch dieses Bild stimmt mit den erwarteten Atompositionen überein. 


\begin{tabular}{|l|l|l|l|l|l|l|}
\hline Pol & $d[\mathrm{~nm}]$ & $d_{\text {theo }}[\mathrm{nm}]$ & $\varphi\left[^{\circ}\right]$ & $\varphi_{\text {theo }}\left[^{\circ}\right]$ & $\frac{E_{\text {Evap }}}{E_{\text {Evap }, 111}}$ & $E_{\text {Evap }}[\mathrm{V} / \mathrm{nm}]$ \\
\hline 110 & $0.2224(2)$ & 0.2234 & 0 & 0.000 & 1 & 57 \\
031 & $0.1045(6)$ & 0.0999 & $26.2(3)$ & 26.6 & $1.025(6)$ & $58.4(3)$ \\
123 & $0.086(1)$ & 0.0845 & $20.4(5)$ & 19.1 & $1.01(1)$ & $57.6(7)$ \\
121 & $0.137(1)$ & 0.129 & $29.4(5)$ & 30.0 & $1.033(16)$ & $58.9(9)$ \\
132 & $0.083(1)$ & 0.0845 & $18.6(5)$ & 19.1 & $0.994(12)$ & $56.6(7)$ \\
112 & $0.130(1)$ & 0.129 & $26(1)$ & 30.0 & $1.006(8)$ & $57.3(4)$ \\
\hline
\end{tabular}

Tabelle 6.1: Winkel $\varphi$ verschiedener Pole zum (11̄0)-Pol sowie Ebenenabstände d und daraus resultierende Feldverdampfungsfeldstärken $E_{\text {Evap. }}$. Die Fehler stammen aus Vergleich der Abstandsbestimmungen mehrere Peaks oder aus dem Fehler der Gaussfits (je nachdem was größer war). Die Fehler der Winkel sind durch die Ableseungenauigkeit bestimmt.

Feldverdampfungsfeldstärke für diese Position aus. Dies führt zu geringeren Ebenenabständen. Der (112)-Pol hingegen ist gut sichtbar und hat entsprechend eine höhere $E_{\text {Evap. }}$. Selbiges gilt in nicht ganz so starkem Maße für den (031)-Pol.

Nach Gleichung 3.3 gilt für den Abstand, den zwei aufeinander folgende Atome in $z$-Richtung zueinander haben, $z_{0} \sim E_{\text {evap }}^{2}$. Alle anderen Parameter die sonst in der Gleichung auftauchen sind innerhalb derselben Messung identisch. Der Geometriefaktor kann allerdings u.U. auch abweichen. Da die Spitzenform in allen von uns gemessenen Spitzen im TEM jedoch gleichmäßig aussah, kann man ihn zu Recht als für alle Positionen auf der Spitze gleichartig annehmen. Deswegen gilt für unterschiedliche Evaporationsparameter $\frac{E_{\text {evap }, 1}}{E_{\text {evap }, 2}}=\sqrt{\frac{z_{0,1}}{z_{0,2}}}$. Zudem ändert sich der mit AtomVicinity festgestellte Ebenenabstand $d$ in Abhängigkeit von $z_{0}$. Es muss allerdings berücksichtigt werden, dass diese Änderung zudem auch noch von der Abweichung des Pols zum Zentrum abhängt. Diese Abhängigkeit wird allerdings bereits durch die Korrektur für kugelförmige Spitzen behoben. Man kann also direkt für den Vergleich der Evaporaporationsfeldstärken der $h k l$ und der $h^{\prime} k^{\prime} l^{\prime}$-Richtung mit den gemessenen Ebenenabständen $d$ und $d^{\prime}$ für einen einheitlichen Gitterparameter $a$ angeben:

$$
\frac{E_{\text {evap }, h^{\prime} k^{\prime} l^{\prime}}}{E_{\text {evap }, h k l}}=\sqrt{\frac{d^{\prime} \sqrt{\frac{h^{2}+k^{2}+l^{2}}{h^{\prime 2}+k^{\prime 2}+l^{\prime 2}}}}{d}}
$$

Es gibt in der LEAP-Messung durchaus statistisch signifikante Unterschiede in den Ebenenabständen, die sich als unterschiedliches Feldverdampfungsverhalten nach Gl. 6.2 interpretieren lassen. Dabei fällt auf, dass in der Richtung, in die verkürzte Winkel gemessen werden $E_{\text {Evap }}$ etwas geringer ist. Dies ist dadurch zu erklären, dass 


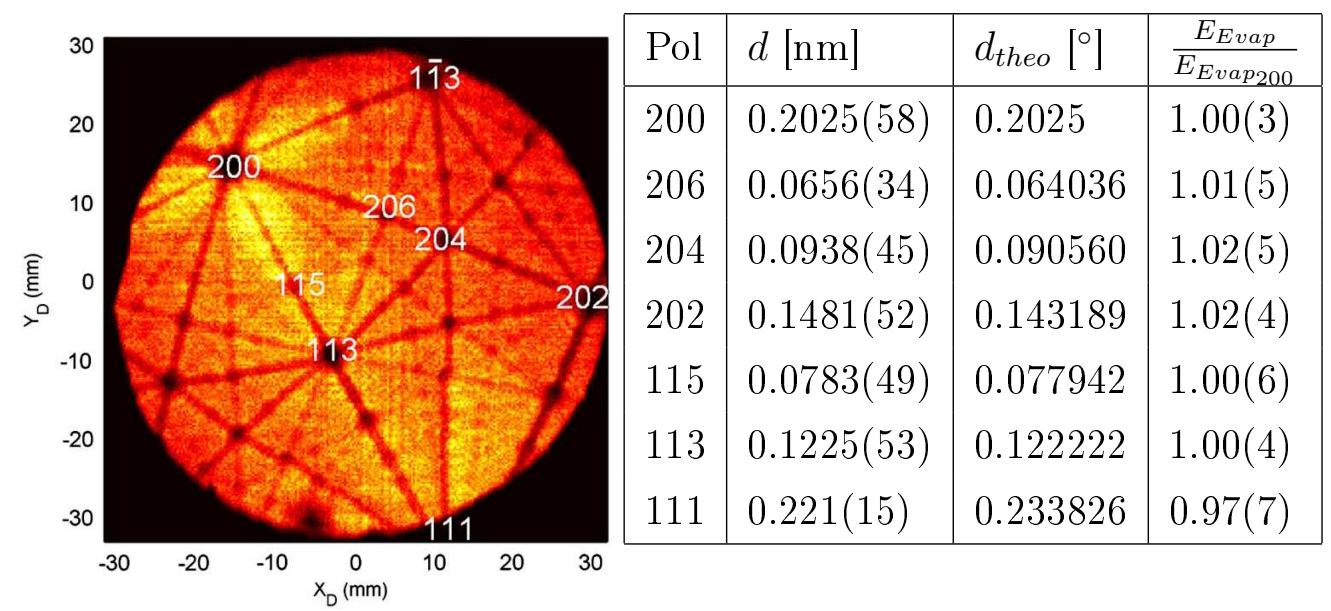

Abbildung 6.14: Al-Messung bei $40 \mathrm{~K}$ von Moody et al. [Mood2009]. In der Abb. links ist das auf dem Detektor gemessene Signal zu sehen. Man sieht im Vergleich zu W erheblich mehr Pole. Die gemessen Ebenenabstände sind von Moody et al. Die Evaporationsfeldstärken wurden nach dem in dieser Arbeit vorgestellten Ansatz berechnet. Der hohe Fehler von Moody erlaubte ihm $E_{\text {Evap }}$ im Sinne der Toleranz als überall gleich anzusehen.

die allgemeine Spitzenkrümmung in diesem Bereich unterschätzt wurde. Die Spitze ist also eigentlich stärker gekrümmt. Ist bereits eine starke Krümmung vorhanden, so ist die zusätzliche Veränderung der Spitzenkrümmung durch einen Pol geringer, was sich in einer scheinbar kleineren $E_{\text {Evap }}$ äußert. Generell folgt die Feldverdampfungsfeldstärke dem zu erwartendem Trend (110), (200), (112), (130), (222), (231) der Prominenz, wobei Prominenz im Fall niedrig indizierter Pole größere, also flache, Pole mit niedrigerer Feldverdampfungsfeldstärke bedeutet. Abweichungen ergeben sich dadurch, dass die Feldverdampfungsunterschiede nicht komplett mit der einfachen Geometrieüberlegung erklärt werden können.

Obwohl Moody et al. [Mood2009] für Aluminium keine Abweichungen der Ebenenabstände berichten, wird bei genauer Betrachtung ihrer Messwerte in Abb. 6.1 deutlich, dass doch zumindest eine Tendenz zu erkennen ist, selbst wenn diese durch hohe Fehler nicht signifikant ist. Die Abfolge der $E_{\text {Evap }}$-Werte entspricht weitgehend der der Prominenz, die nach [Mil11996] für kfz-Gitter zu erwarten ist, nämlich (111), (200), (220), (113), (240). Hierbei bedeutet Prominenz wieder große, flache Pole, also eine geringere Feldverdampfungsfeldstärke. Die statistisch größere Signifikanz der Unterschiede von $E_{\text {Evap }}$ für verschiedene Richtungen bei Wolfram lässt sich durch den erheblich höheren Schmelzpunkt von Wolfram (3700 K) gegenüber Aluminium (930 K) erklären. Der Einfluss der Temperatur auf das Feldverdampfungsverhalten ist bei $40 \mathrm{~K}$ auf Aluminium somit weit größer.

Obwohl der Einfluss der globalen Geometrie auf den Winkel und der der lokalen 

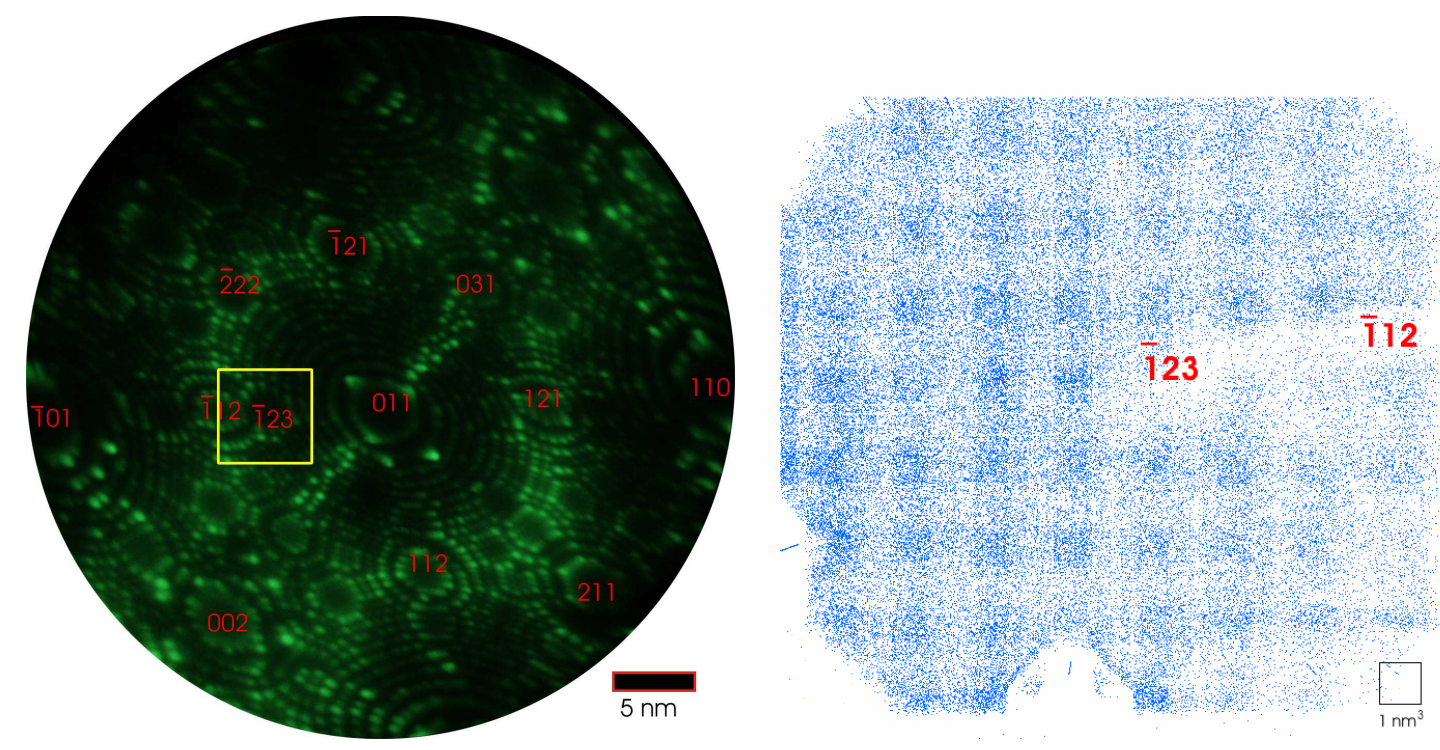

Abbildung 6.15: FIM-Aufnahme einer Wolframspitze (links). Gelb markiert ist der Bereich, von dem später eine TAP-Messung (rechts) angefertigt wurde. Die Ecken am unteren linken Rand der TAP-Messung stammen von zwei defekten Detektoranoden. Man sieht, dass der [123] und [112]-Pol durch einen ausgedünnten Bereich verbunden sind, wie es sich auch im FIM-Bild bereits andeutet.

Geometrie auf die Ebenenabstände hier als störend erscheint, lässt er sich dennoch nicht nur in den Simulationen nachstellen (Kap. 7.4) sondern im Prinzip sogar zur Kalibrierung der Simulationsparameter verwendet werden. Zudem erlaubt er Aussagen über die lokal unterschiedlichen Feldverdampfungsfeldstärken (Kap. 6.4.5).

\subsubsection{W-Messung mit TAP}

In Abb. 6.15 ist eine FIM-Aufnahme der untersuchten Wolframspitze zu sehen. Man beachte, dass der untersuchte (123)-Pol sich mit dem (112) und dem (011)-Pol in einer Linie befindet, die parallel zur $x$-Achse ist. Entsprechend findet man in der Aufsicht vom TAP den (123) in der Mitte und rechts ${ }^{4}$ von ihm den (123)-Pol. In Abb. 6.16 findet man eine Darstellung der AtomVicinity-Ergebnisse als Dichteverteilung. Man erkennt in der $(x, z)$-Schnittebene, dass sich die Ebenen in [011]-Richtung in feinere um $16^{\circ}$ verkippte Ebenen aufspalten. Diese feinen Ebenen resultieren aus dem in der Mitte der Probe gelegenen [123]-Pol. Die (011)-Ebenen sind trotz der Entfernung des Analysevolumens zum (011)-Pol zu erkennen, da diese sehr prominent sind. In der Tat kann man auch an den im TAP-Volumen gelegenen Polen noch Ringe erkennen, die zum (011)-Pol gehören. Durch die Überlagerung der Ebenen

\footnotetext{
${ }^{4}$ Man beachte, dass das FIM-Bild über einen Spiegel aufgenommen wurde.
} 


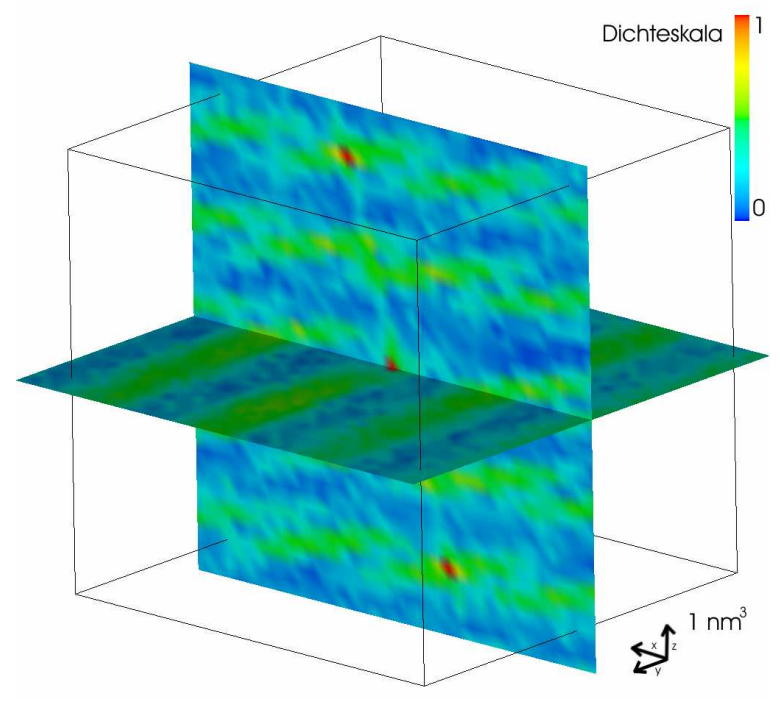

(a) $(y, z)$ - und $(x, y)$-Flächendichteprofile

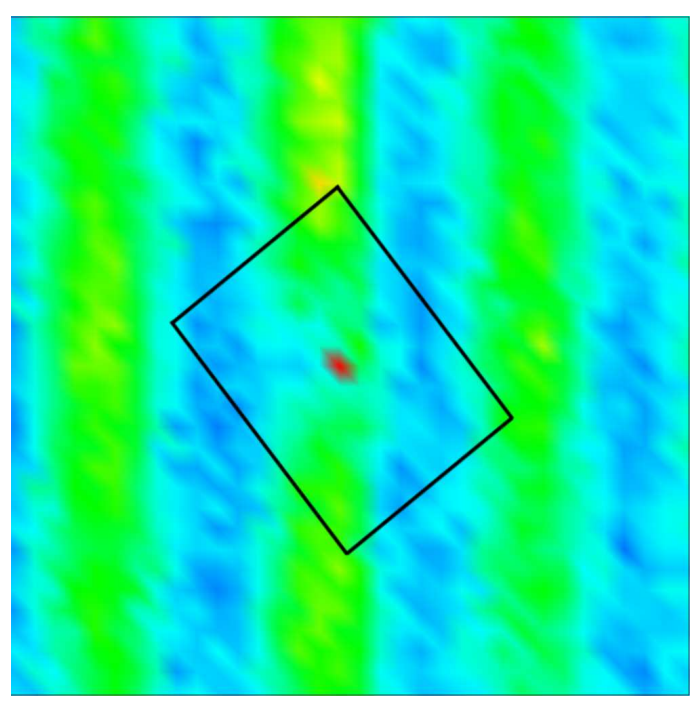

(b) $(x, y)$-Flächendichteprofil um das Zentrum herum

Abbildung 6.16: AtomVicinity-Flächendichteprofil der gesamten TAP-Messung aus Abb. 6.15 in Falschfarbendarstellung mit 1 normiert auf die höchste Dichte abseits des Zentrums. Die Box enthält ein Volumen von $1 \mathrm{~nm}^{3}$. Die z-Richtung ist die [011]-Richtung. Zusätzlich zu diesen großen Ebenenabständen kann man in der $(x, z)$-Schnittebene kleine Ebenenabstände erkennen. In der (x,y)-Ebene um das Zentralatom herum (rechts) sieht man, dass einzelne Atompositionen erkennbar sind, die leider von den durch die Ecken des eingezeichneten Vierecks dargestellten erwarteten Positionen abweichen. Die Darstellung ist also in $(x, y)$-Richtung gedehnt.

mehrerer Pole erhält man somit auch im TAP die Möglichkeit in eine der lateralen Richtungen Ebenen unterscheiden zu können. Dies ist besonders gut in der Aufsicht in Abb. 6.16(b) zu erkennen. Dort kann man allerdings auch erkennen, dass die $(x, y)$-Längenskala nicht stimmen kann. Die grünen Balken, die von unten nach oben ragen, sind, da sie senkrecht zu einer $<110>$-Richtung stehen, wiederum eine $<110>$-Richtung, müssten also dieselben Ebenenabstände haben.

Misst man die Ebenenabstände $d$ der einzelnen Pole und die Winkel $\varphi$ zur [011]Richtung, so erhält man die Ergebnisse in Tabelle 6.2

\begin{tabular}{|l|l|l|l|l|}
\hline Pol & $d[\mathrm{~nm}]$ & $d_{\text {theo }}$ & $\varphi\left[^{\circ}\right]$ & $\varphi_{\text {theo }}\left[^{\circ}\right]$ \\
\hline 110 & $0.224(2)$ & 0.223 & - & - \\
$\overline{1} 12$ & $0.156(2)$ & 0.1290 & $18.0(5)$ & 30.0 \\
$\overline{1} 23$ & $0.092(1)$ & 0.0845 & $15.8(5)$ & 19.1 \\
\hline
\end{tabular}

Tabelle 6.2: Ebenenabstände und Winkel von $W$ im TAP (40 K $\left.U_{P u l s}=0.2 U_{B a s i s}\right)$

Die Fehler der Ebenenabstandsmessungen stammen aus den Gaussfits an den Atom- 
Vicinity-Dichteprofilen in die entsprechenden Richtungen. Der Fehler für den Winkel ergibt sich aus der Ungenauigkeit der händischen Einstellung, die über eine Optimierung der W-Peakhöhen der AtomVicinity-Dichteprofile ermittelt werden kann. Die Peaks sind am höchsten und am besten zu unterscheiden, wenn die Messung so verkippt wird, dass die Ebenen senkrecht zur Dichteprofilrichtung stehen.

Die theoretischen Ebenenabstände ergeben sich aus dem Gitterparameter $a=0.316$ nm für Wolfram [Mill1996]. Man erkennt dass die Ebenenabstände stark abweichen. In [123]-Richtung hingegen ist eine Abweichung unverkennbar. Die Winkel weichen ebenfalls für beide Richtungen stark ab.

Die Ursache liegt vermutlich darin, dass in der Nähe eines (011)-Pols die Umgebung stark abgeflacht ist. Da der Rekonstruktionsalgorithmus auf den Spitzenradius Bezug nimmt, findet eine Korrektur der z-Positionen der Atome für einen falschen, nämlich einen zu großen, Radius statt. Dies lässt sich in Abb. 6.16(b) gut erkennen, in denen die rekonstruierten $(x, y)$-Positionen eindeutig zu groß sind, wie man es durch Annahme einer zu kleinen Vergrößerung, die nach Gl. 3.1 bei einem zu großen Spitzenradius entsteht, erwartet. Die z-Positionen werden bei der Korrektur nach Gl.3.4 mit einem zu großen Radius deshalb nicht so stark in $z$-Richtung verschoben, wie sie es normalerweise würden. Dadurch werden die Ebenen nicht genug gebogen und der resultierende Winkel ist zu klein.

Besonders ins Auge fällt der dramatische Unterschied der gemessenen Ebenenabstände für die LEAP-Messung in Tabelle 6.1 im Vergleich zur TAP-Messung in Tabelle 6.2. Die unterschiedlichen Winkel zur [011]-Richtung lassen sich noch vergleichsweise einfach erklären, wenn man die Art der Bestimmung in der TAP-Messung berücksichtigt. Hier wurde die(011)-Ebene im Gegensatz zur LEAP-Messung nicht am dazugehörigen Pol identifiziert. Sie war nur aufgrund ihrer starken Prominenz noch im TAP-Messausschnitt feststellbar. Bestimmt man die Ebene für das LEAP ebenso, so erhält man für den (123)- und (121)-Pol Winkel von $(15.6 \pm 2)^{\circ}$ und $(24.4 \pm 2)^{\circ}$. Zumindest der Winkel zum (123)-Pol ist somit erklärbar. Der Winkel zum (121)-Pol weicht hingegen ab. Betrachtet man die Winkel des (132)- und (112)-Pols in gleicher Weise, so erhält man Winkel von $(12.4 \pm 2)^{\circ}$ und $(19.8 \pm 2)^{\circ}$. Hier stimmt der Unterschied der beiden Winkel zueinander allerdings ebenfalls nicht. Zudem sind die Abweichungen in der LEAP-Messung nur in dem sehr kleinen Bereich um die beiden Pole vorhanden. Wählt man einen mit dem TAP-Analysebereich vergleichbaren Bereich aus, so ändert sich der LEAP-Winkel zu den in der Tabelle aufgetragenen Werten nicht. Die einzige sinnvolle Erklärung ist, dass die Rekonstruktion der TAP- 
Messung in der bisherigen Form falsch ist. Dies ist auch durch die nicht stimmigen Gitterparameter in $(x, y)$-Richtung in Abb. 6.16(b) offensichtlich. Deswegen wurde die TAP-Rekonstruktion mit einem sinnvollen Wert von $\xi=1.4 . .1 .8$ (in Gl. 3.1) variiert. Tatsächlich erreicht man dadurch eine sehr gute Übereinstimmung der Winkel für sinnvolle Werte von $E_{\text {Evap }} \beta$. Jedoch ändern sich die Ebenenabstände nicht nur für den (110)-Pol, sondern auch für den im Zentrum der Analyse befindlichen Pol. Über das auszufüllende Volumen ist $z$ nach Gl. 3.3 indirekt mit den $(x, y)$-Werten verbunden. Es ändert sich sowohl $z \sim\left|x_{\min }-x_{\max }\right|$ als auch $z \sim\left|y_{\min }-y_{\max }\right|$ und somit ist $z \sim \xi^{2}$. Es ist für sinnvolle Parameter von $\xi$ nicht möglich, passende Ebenenabstände, wie man sie nach der LEAP-Messung erwartet, zu erhalten. Im Grunde müsste ein Stauchungsfaktor für die $(x, y)$-Richtung verwendet werden, wie es Geiser et al. [Geis2007] vorschlagen. Ursache für das Versagen des üblichen Rekonstruktionsalgorithmus dürfte die Ausdünnung der Dichte im Bereich der im Analysevolumen befindlichen Pole sein. Um die Abstände korrekt zu rekonstruieren wird ein, für die an den Polen bestimmten Ebenenabstände passendes Atomvolumen, angenommen. Da dort jedoch ein lokaler Vergrößerungseffekt herrscht, ist dieses Volumen für den Rest der Messung zu groß und verursacht die beobachtete Dehnung in $(x, y)$-Richtung. Dadurch, dass im LEAP die (110)-Ebenen auch weit außerhalb des ausgedünnten Bereichs erkennbar waren und somit ein großes Volumen für die Kalibrierung benutzt werden konnte, spielt dieser Effekt dort nur eine geringe Rolle.

\subsubsection{Feldverdampfungsfeldstärken in $\mathrm{Ti}_{51} \mathrm{Al}_{48} \mathrm{Nb}_{1}$}

Auch in einer LEAP-Messung von $\mathrm{Ti}_{51} \mathrm{Al}_{48} \mathrm{Nb}_{1}$ lässt sich der Einfluss der im untersuchten Volumen befindlichen Pole auf den AtomVicinity-Algorithmus überprüfen. Dabei stellt man in der Tat einen Einfluss auf die gemessenen Ebenenabstände und Winkel fest, die auf eine Veränderung der Evaporationsfeldstärke zurückzuführen sind. In Abb. 6.17 sind die FIM-Aufnahme und die von oben betrachtete LEAP-Messung abgebildet. Der später im LEAP gemessene Bereich ist in der FIM-Abbildung eingezeichnet. Das originale FIM-Bild wurde an der $(y, z)$-Ebene gespiegelt. (Dies wurde nur in diesem Bild gemacht, da hier sonst ein Vergleich mit dem LEAP schwierig ist. Generell wurde sonst überall ein ungespiegeltes FIM-Bild verwendet ${ }^{5}$.

\footnotetext{
${ }^{5} \mathrm{Da}$ die gespiegelten Pole ebenso auftauchen wie die ungespiegelten spielt dies sonst keine Rolle, obwohl es streng genommen falsch ist.
} 


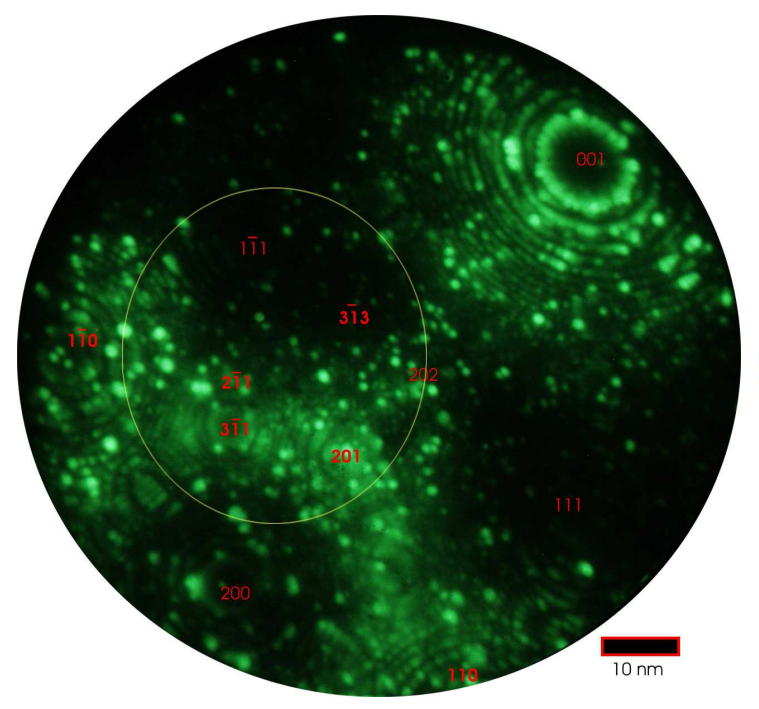

(a) FIM

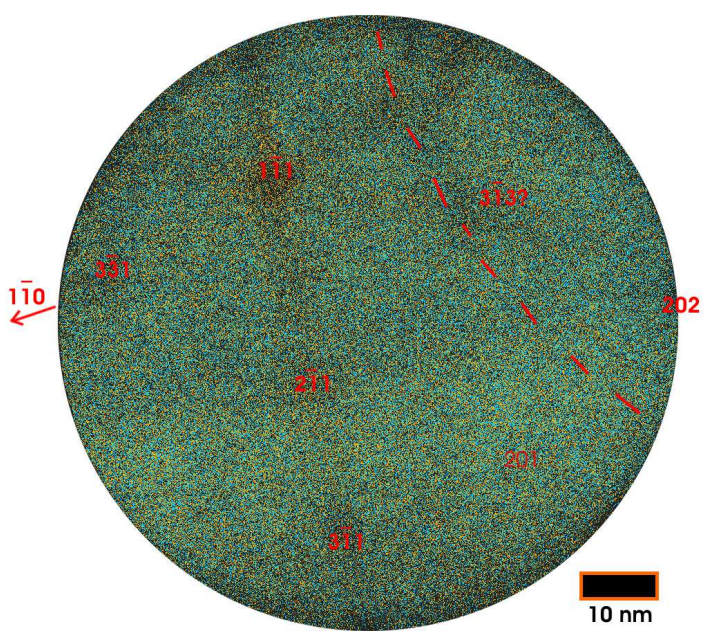

(b) LEAP

Abbildung 6.17: Indizierung und Auswahl im Göttinger FIM (40 K, $p_{\text {Neon }}=10^{-4}$ mbar $)$ und der späteren Messung im LEAP (38 K, ohne Laser. Die Pole sind teilweise nicht zu erkennen, wurden aber dennoch dort ergänzt. Der gelbe Kreis in (a) umrandet den Bereich, der in (b) gemessen wurde. Man beachte, dass der Bereich gegenüber der LEAP-Messung verdreht ist. Das originale FIM-Bild wurde zudem an der $(y, z)$-Ebene gespiegelt. Die gestrichelte Linie rechts oben bezeichnet einen leicht ausgedünnten Bereich, der vermutlich durch eine Kleinwinkelkorngrenze verursacht wird. Der (110)-Pol liegt, außerhalb des gemessenen LEAP-Volumens, in der durch einen roten Pfeil angegeben Richtung. 
Die Ergebnisse der Berechnung von $E_{\text {Evap }}$ nach Gl. 6.2 sind in Tabelle 6.3 aufgetragen. Man erkennt, dass ein erheblicher Einfluss auf das Evaporationsverhalten vorliegt, der bis zu einem Unterschied von $15 \%$ führen kann.

Der Wert des (31)3) ist vermutlich durch die Lage auf der Korngrenze verfälscht. Es liegt möglicherweise auch ein (211)-artiger geordneter Pol vor, was möglich wäre, da evtl. ein Spitzenabriss vor Beginn der Messung stattgefunden hat. Diese Annahme ist in der Tabelle ebenfalls eingetragen, liefert aber auch einen stark verfälschten Wert, der allerdings plausibler ist, weil ein niedriges $E_{\text {Evap }}$ mit dem dort vorliegenden dunklen Pol gut vereinbar ist. Andere niedrigindizierte Pole in der Umgebung lassen sich mit großer Wahrscheinlichkeit ausschließen. Ansonsten hätte die Korngrenze besser sichtbar sein müssen. Für die $\{110\}$-Richtungen sind die Fehler groß, da sich beide außerhalb des gemessenen Volumens befinden und nur aufgrund ihrer großen Dominanz gemessen werden konnten. Die Winkel für einige Pole passen, wenn man annimmt, dass sie hinter einer Korngrenze mit ca. $4^{\circ}$ Verkippung liegen. Der geordnete (110)-Pol besitzt einen stark abweichenden Winkel, welcher allerdings, analog zu den Beobachtungen für Winkelmessungen abseits des Pols für Wolfram, plausibel ist.

\begin{tabular}{|l|l|l|l|l|l|l|}
\hline Pol & $d[\mathrm{~nm}]$ & $d_{\text {theo }}[\mathrm{nm}]$ & $\varphi\left[^{\circ}\right]$ & $\varphi_{\text {theo }}\left[^{\circ}\right]$ & $\frac{E_{\text {Evap }}}{E_{\text {Evap, } 1 \overline{1} 1}}$ & $E_{\text {Evap }}[\mathrm{V} / \mathrm{nm}]$ \\
\hline $1 \overline{1} 1$ & $0.221(1)$ & 0.231 & 0 & 0 & 1 & $21.4(1)$ \\
$1 \overline{1} \overline{2}$ & $0.183(1)$ & 0.1633 & $18.2(3)$ & 19.47 & $1.08(1)$ & $23.2(2)$ \\
$3 \overline{1} 1$ & $0.157(1)$ & 0.121 & $30.6(3)$ & 29.47 & $1.17(1)$ & $25.0(2)$ \\
$3 \overline{3} 1$ & $?$ & & $16.2(5)$ & 22.0 & - & - \\
$3 \overline{1} 3 ?, \mathrm{KG}$ & $0.140(2)$ & 0.0917 & $18.4(3)$ & 22.0 & $1.26(9)$ & $27.0(4)$ \\
$1 \overline{1} 0$ & $0.31(5)$ & 0.282 & $20(1)$ & 35.3 & $1.07(17)$ & $23(4)$ \\
$101, \mathrm{KG}$ & $0.26(5)$ & 0.2 & $40(2)$ & 35.3 & $1.17(23)$ & $25(5)$ \\
$201, \mathrm{KG} ?$ & $0.21(1)$ & 0.179 & $34(2)$ & 39.2 & $1.11(5)$ & $24(2)$ \\
$3 \overline{1} 3$ als 211 & $0.140(2)$ & 0.163 & $18.4(3)$ & & $0.94(7)$ & $20(2)$ \\
\hline
\end{tabular}

Tabelle 6.3: Winkel $\varphi$ verschiedener Pole zum (11̄1)-Pol sowie Ebenenabstände d und daraus resultierende Feldverdampfungsfeldstärken $E_{\text {Evap }} . K G$ bezeichnet die Pole, die sich auf der anderen Seite der Korngrenze befinden. Der (31̄3)-Pol ist möglicherweise ein geordneter (211)-Pol, wie in der letzten Zeile angedeutet wird.

Es gibt bei dieser Methode zwei mögliche Ungenauigkeiten. Zum einen ist der Gitterparameter, aufgrund der Stauchung der Einheitszelle, für verschiedene Richtungen unterschiedlich. Die Abweichung liegt allerdings bei nur etwa 1.5\%, sodass es für 
die betrachteten Indexierungen, die größtenteils in etwa in die [111]-Richtung zeigen, keinen wesentlichen Unterschied ausmachen. Zum anderen kann die Annahme halbkugelförmiger Spitzen eventuell nicht mehr ausreichen. Dies, sowie ein Fehler des Rekonstruktionsalgorithmus, kann als Einfluss ausgeschlossen werden, da von Michael Moody et. al [Mood2009] für Aluminium nachgewiesen wurde, dass die Unterschiede zwischen theoretisch erwarteten und gemessenen Ebenenabständen unter $0.002 \mathrm{~nm}$ liegen. Die von Moody gemessenen Winkel weichen um bis zu $2^{\circ}$ für einen $30^{\circ}$-Winkel von den erwarteten Winkeln ab. Unsere W-Messung in Tabelle 6.1 zeigt ein vergleichbares Verhalten. Die für $\mathrm{Ti}_{51} \mathrm{Al}_{48} \mathrm{Nb}_{1}$ gemessenen Winkelabweichungen in Tabelle 6.3 sind teilweise größer. Allerdings ist die Abweichung dadurch bedingt,

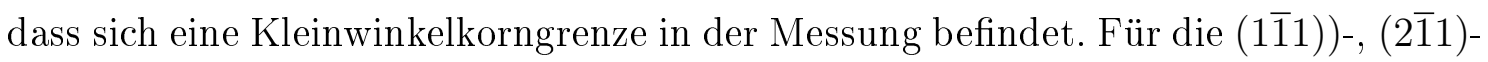
sowie den (31̄1)-Pol, die nicht durch eine Kleinwinkelkorngrenze getrennt werden, liegt die Abweichung im gleichen Bereich ${ }^{6}$. Der (201)-Pol sollte nach der Skizze der Korngrenze auch nicht betroffen sein. Jedoch ist die genaue Position der Korngrenze schlecht zu bestimmen.

Die Werte $E_{\text {Evap }}$ scheinen vor allem deswegen plausibel, da Pole, die ein hohes $E_{\text {Evap }}$ besitzen im FIM-Bild ebenfalls hell erscheinen. Dunkle Pole, wie der (111)Pol, haben niedrige $E_{\text {Evap }}$.

\subsubsection{Einfluss von Laserenergie und Temperatur}

Um den Einfluss der Messparameter für die Laseratomsonde zu bestimmen wurde für $\mathrm{Ti}_{51} \mathrm{Al}_{48} \mathrm{Nb}_{1}$ eine Messreihe mit verschiedenen Laserenergien und Temperaturen durchgeführt. In verschiedenen Messungen wurden dabei große Mengen Hydride gemessen. In den verschiedenen Messungen variierte die Anzahl der Hydride, die vor allem in den ersten Laser-Messungen detektiert wurden. Im weiteren Verlauf nahm ihr Anteil ab. Die Hydride resultieren wahrscheinlich aus wasserstoffhaltigen Verbindungen (verm. vor allem Wasser und evtl. Kohlenwasserstoffe) vom Probenschaft, die vom Laser erwärmt und dadurch mobil wurden. Durch Oberflächenmigration konnten sie zur Probenspitze gelangen. Dieser Effekt lässt sich nicht nur durch den Laser erzeugen. Erhöht man die Temperatur, so werden die Wasserstoffverbindungen ebenfalls mobil und können an der Spitze Hydride entstehen lassen. Im Laufe der Zeit wird dieser Vorrat aufgebraucht.

\footnotetext{
${ }^{6}$ Die Stauchung der Einheitszelle bewirkt eine Abweichung der Winkel von maximal 0.5 und kann somit vernachlässigt werden.
} 
Ein Einfluss des Lasers auf die gemessenen Ebenenabstände und Winkel zeigt sich ebenfalls. Die ursprüngliche Hoffnung war, mit dem Laser das Feldverdampfungsverhalten der beiden Partner aneinander anzugleichen. In der Tat ist bei der niedrigsten

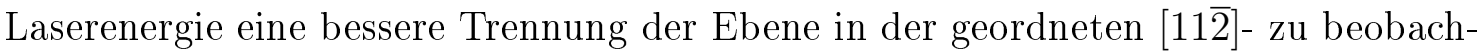
ten. Bei genauerer Betrachtung konnte ein solches Aufspalten der Positionen jedoch auch an den anderen Polen beobachtet werden. Der Effekt war also sogar gegenteilig und sorgte dafür, dass zunächst alle Atome der einen Komponente aus der Oberfläche herausgelöst wurden, bevor die andere Komponente mit der Feldverdampfung beginnt. Bei höheren Laserenergien trat dieser Effekt nicht mehr auf. Zudem erkauft man sich die bessere Trennbarkeit der Ebenen mit einer sehr viel schlechteren Auflösung. Die Peakpositonen sind wesentlich ungenauer zu bestimmen. Zudem sind die Peaks wesentlich breiter. In Tabelle 6.4 ist der Einfluss der Messreihe mit verschieden Laserenergien und verschiedenen Temperaturen dargestellt. Offenbar beeinflusst der Laser sowohl die Ebenenabstände $d$ als auch den Winkel $\varphi(111,11 \overline{2})$ zwischen dem (111) und (11 $\overline{2})$-Pol. Die Messungen wurden jeweils so rekonstruiert, dass die Ebenenabstände des (111)-Pols stimmen. Da jedoch das notwendige Auswerteprogramm für das nicht öffentliche LEAP-Datenformat von Imago in Göttingen nicht zur Verfügung stand, konnten die Einstellungen nicht in allen Fällen mit der üblichen Präzision erfolgen. Die Ebenenabstände der (111)-Ebene sind deswegen ebenfalls angegeben.

In Abb. 6.18 ist zu sehen, dass der Laser die Spitzengeometrie verändert. Dies geschieht auf der Größenskala der ganzen Spitze. Kleine Assymetrien, die vorher etwa an den Polen vorlagen, werden zerstört. Dies äußert sich in veränderten Ebenenabständen während der Messung. Dieser Effekt ist wahrscheinlich nicht stabil. Wie man an der Messung der Ebenenabstände für $0.24 \mathrm{~nJ}$ erkennen kann, bedeutet eine höhere Laserenergie nicht zwangsläufig eine Verringerung der Ebenenabstände. Da die Spitze vorher längere Zeit mit unwesentlich geringeren Laserenergien verändert wurde, beginnt sich wieder ein Gleichgewicht einzustellen, bei dem die lokale Probengeometrie eine von der Umgebung abweichende Feldverdampfung am Pol zulässt. Die berechneten $E_{\text {Evap }}$ sind, da keine stabile Spitzengeometrie vorliegt, somit nicht als Feldverdampfungsfeldstärken für entsprechende Laserenergien und Temperaturen zu bewerten, sondern lediglich als Hinweis auf die jeweilige Spitzenverformung. 


\begin{tabular}{|l|l|l|l|l|l|}
\hline$T[\mathrm{~K}]$ & Laserenergie [nJ] & $\varphi(111,11 \overline{2})\left[^{\circ}\right]$ & $d_{111}[\mathrm{~nm}]$ & $d_{11 \overline{2}}[\mathrm{~nm}]$ & $\frac{E_{\text {Evap }, 11 \overline{2}}}{E_{\text {Evap }, 111}}$ \\
\hline 38 & 0 & $18.2(5)$ & $0.221(1))$ & $0.183(1)$ & $1.08(1)$ \\
35 & 1.155 & $17.1(3)$ & $0.221(1)$ & $0.173(2)$ & $1.05(1)$ \\
38 & 0.188 & $16.4(5)$ & $0.236(1)$ & $0.161(2)$ & $0.98(1)$ \\
38 & 0.22 & $17.1 \pm 1$ & $0.217(1)$ & $0.166(1)$ & $1.04(1)$ \\
38 & 0.24 & $17.3 \pm 1$ & $0.225(1)$ & $0.175(2)$ & $1.05(1)$ \\
58 & 0.28 & $17.1(5)$ & $0.226(1)$ & $0.165(2)$ & $1.02(2)$ \\
38 & 0.35 & $16.9 \pm 1$ & $0.238(1)$ & $0.133(1)$ & $0.89(1)$ \\
54 & 0.18 & $18.1(5)$ & $0.238(1)$ & $0.199(5)$ & $1.09(3)$ \\
54 & 0 & $19.8(5)$ & $0.222(2)$ & $0.197 \pm 0.016$ & $1.12(9)$ \\
94 & 0 & $19.8(3)$ & $0.217(2)$ & $0.169(1)$ & $1.05(1)$ \\
94 & 0.18 & $20.0(5)$ & $0.218(3)$ & $0.150(6)$ & $0.99(4)$ \\
& theoretisch & 19.471 & 0.231 & 0.1633 & 1 \\
\hline
\end{tabular}

Tabelle 6.4: Der Einfluss der Messreihe auf Ebenenabstände und Winkel. Für die Messungen ohne Laser beträgt $U_{\text {Puls }}=0.23 U_{\text {Basis. }} . E_{\text {Evap }, 11 \overline{2}} / E_{\text {Evap }, 111}$ ist zur Verdeutlichung des Einflusses einer Veränderung der Messparameter angegeben. Da die Spitzen während der Messung nicht geometrisch stabil waren, beschreiben sie nicht tatsächlich die Evaporationsfeldstärken. Die Fehler der Ebenenabstände resultieren aus den Gaussfits oder aus der Standardabweichung verschiedener Abstandsbestimmungen (je nachdem welcher größer war). Die Fehler der Winkelmessung basieren auf der erreichbaren Ablesegenauigkeit. Andere Fehler stammen aus der Fehlerfortpflanzung.
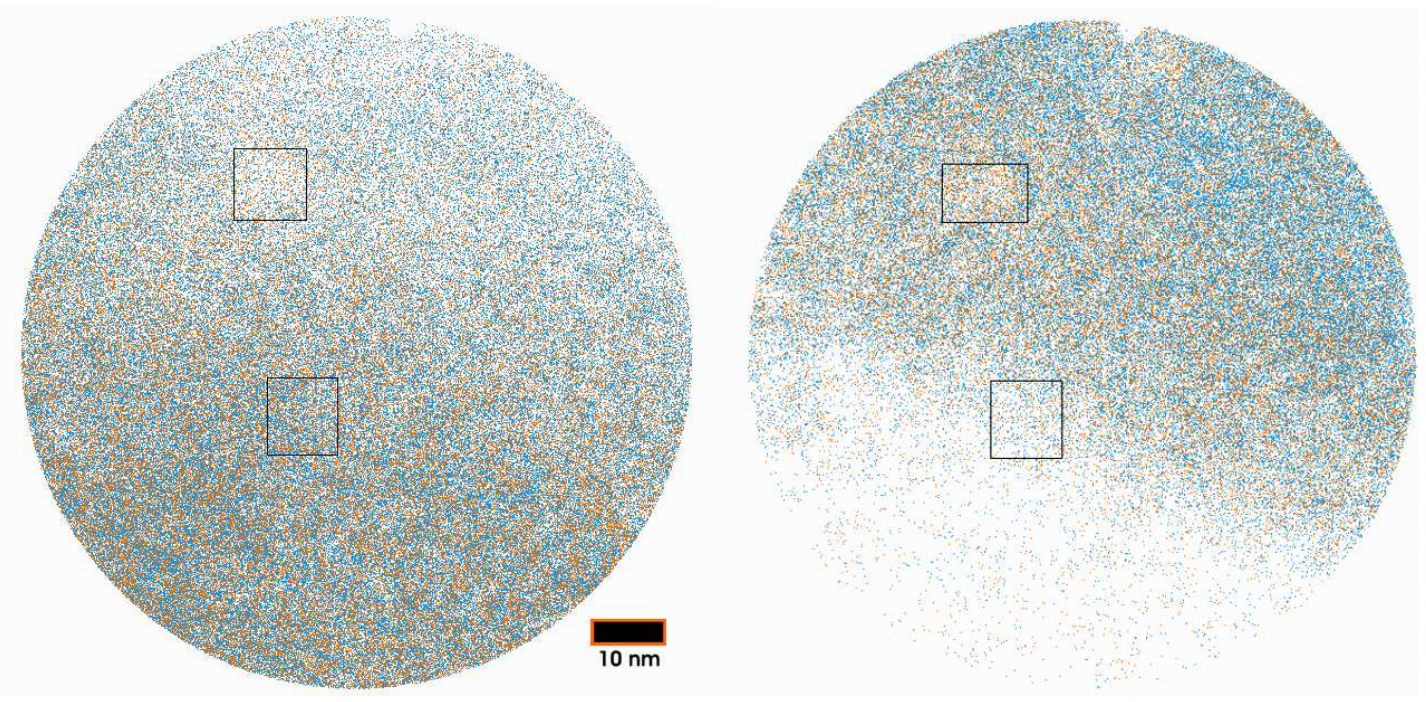

Abbildung 6.18: Aufsicht auf die Messungen bei $38 \mathrm{~K}$ mit einer Laserenergie von 0.24 $n J$ (links) und nach Abschalten des Lasers bei $54 K$ mit $U_{P u l s}=0.23 U_{\text {Basis }}$ (rechts). Der Laser trifft offenbar auf den unteren linken Teil der Probe. Deswegen findet hier verstärkte Feldverdampfung statt, solange der Laser aktiviert ist. Nach Abschalten des Lasers stellt sich langsam wieder eine symmetrische Spitzenform ein. Dies führt zu erhöhter Feldverdampfung in dem Bereich, der vorher nicht so stark vom Laser beeinflusst war. 


\subsection{TAP: Mikrostruktur}

In keiner der durchgeführten TAP-Messungen von $\mathrm{Cu}_{3}$ wurden Korngrenzen beobachtet, da diese mit $800 \mathrm{~nm}$ Durchmesser (Kap. 6.2.2) größer sind als als der mit dem TAP analysierte Bereich und zudem solche Messungen nicht angestrebt wurden. Wahrscheinlich sind Korngrenzen für die Mehrzahl der Spitzenabrisse während der TAP-Messung mitverantwortlich. Domänengrenzen sollten nach Röntgen- und FIMAnalysen (Kap 6.2.2,6.3.2) hingegen in den TAP-Messungen häufig sein. Allerdings sind sie in unseren Messungen praktisch nicht zu beobachten. Gut zu beobachten wären sie, wenn große Feldverdampfungsunterschiede vorliegen und die Dömanengrenze nicht senkrecht zur Analyserichtung steht. Während Letzteres meist der Fall sein sollte, ist die Feldverdampfungsfeldstärke auf längere Skalen gesehen identisch. Lediglich direkt an der Domänengrenze, an der entweder zu viele $\mathrm{Cu}$ - oder zu viele Au-Atome sitzen, ist bestenfalls eine leichte Abweichung feststellbar. In Abb. 6.19 ist eine Stelle, an der mutmaßlich dieser Effekt auftritt, abgebildet. Eine sichere Identifizierung ist allerdings nicht möglich, lediglich die Häufigkeit, mit der diese geringen Dichteschwankungen festgestellt werden können, stimmt mit der erwarteten Domänengröße überein. Für die Ergebnisse von AtomVicinity-Dichteprofilen sind diese Domänengrenzen, solange sie nicht zu häufig auftreten, nicht von Bedeutung.

Für TiAl sind einerseits $\alpha / \gamma$-Phasengrenzen feststellbar, andererseits erwartet man wiederum Domänengrenzen. Hier gibt es zwei Fälle. Einerseits können, wie bei $\mathrm{Cu}_{3} \mathrm{Au}$, die beiden Domänen gleich orientiert und lediglich ein kleiner Effekt durch überzählige Ti- oder Al-Atome beobachtbar sein. Es kann aber auch eine Domänengrenze vorhanden sein, an der ein Übergang von einer geordneten in eine nichtgeordnete Richtung stattfindet. In einem solchen Fall sorgt ein großer Unterschied in den Feldverdampfungsfeldstärken für eine gut sichtbare Domänengrenze (Abb. 6.20).

Zwillingsgrenzen sind ein weiterer Grenzflächentyp, der in in den TiAl-Legierungen beobachtet werden kann. In Abb. 6.21 ist eine solche Grenze, wie sie in [Boll2005] gemessen wurde, dargestellt. Die im Konzentrationsprofil beobachtete Nb-Konzentration an der Grenzfläche ist erhöht. Daraus kann man, aufgrund des unterschiedlichen Feldverdampfungsverhaltens der Elemente, allerdings nicht direkt auf eine tatsächlich höhere Nb-Konzentration schließen. 


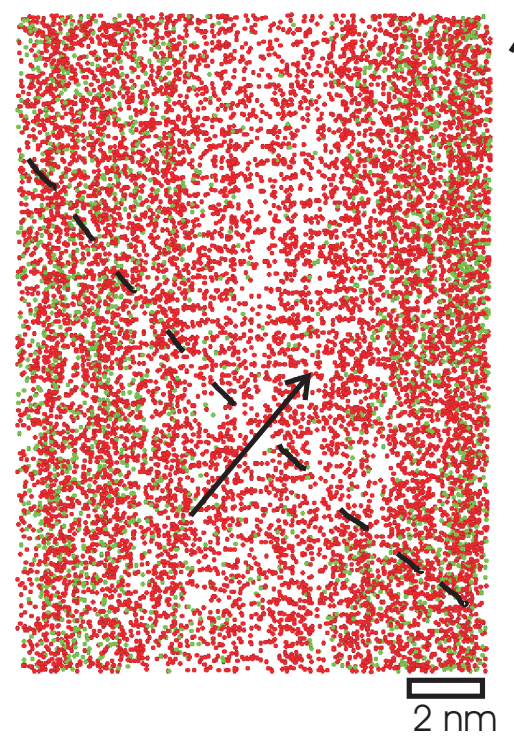

\section{个[1 10] \\ $\mathrm{Cu}$ $\mathrm{Au}$}

Abbildung 6.19: Cu $u_{3} A$-Messung in [110]-Richtung (links). Es ist an der Position der gestrichelten Linie ein Bereich leicht ausgedünnter Dichte und weniger gut unterscheidbarer Netzebenebenen zu beobachten, der als Domänengrenze interpretiert werden kann. Betrachtet man ein Konzentrationsprofil (rechts) in Richtung des eingezeichneten Pfeils, so erhält man einen Bereich leicht geringerer Au-Dichte. Dies ist allerdings weniger ein Effekt der Domänengrenze als vielmehr der geringen Au-Atomdichte in der Mitte des Pols durch den lokalen Vergrößerungseffekt (siehe Kap. 6.6). Ebenfalls gut erkennbar ist, dass die Netzebenen im ausgedünnten Bereich in der Mitte (im Zentrum des Pols) wesentlich besser unterscheidbar sind als am Rand.

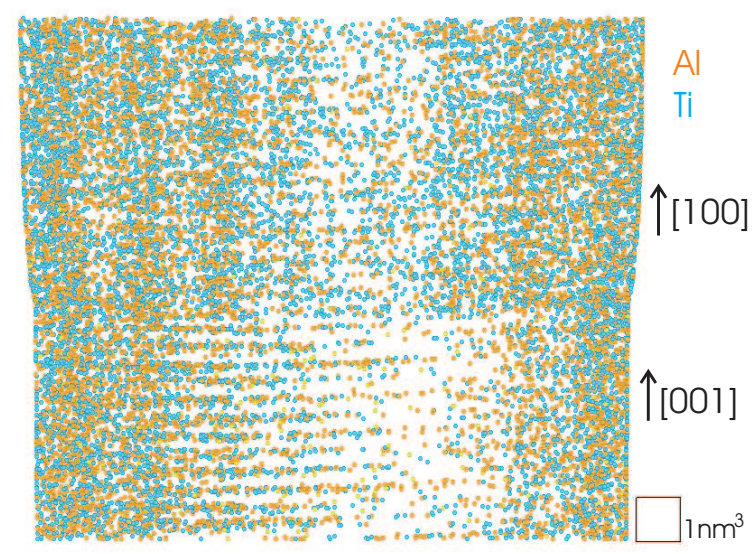

Abbildung 6.20: Ti50.2at.\%Al2.1at.\%Cr-Messung mit [001]/[100]-Domänenegrenze. Der gut sichtbare Pol mit (001)-Ebenen geht in eine (100)-Pol über, dessen Ebenen weniger gut sichtbar sind. In die [001]- Überstrukturrichtung liegen scheinbar Ebenen in doppelt so großen Abständen wie in die [100]-Richtung vor. 


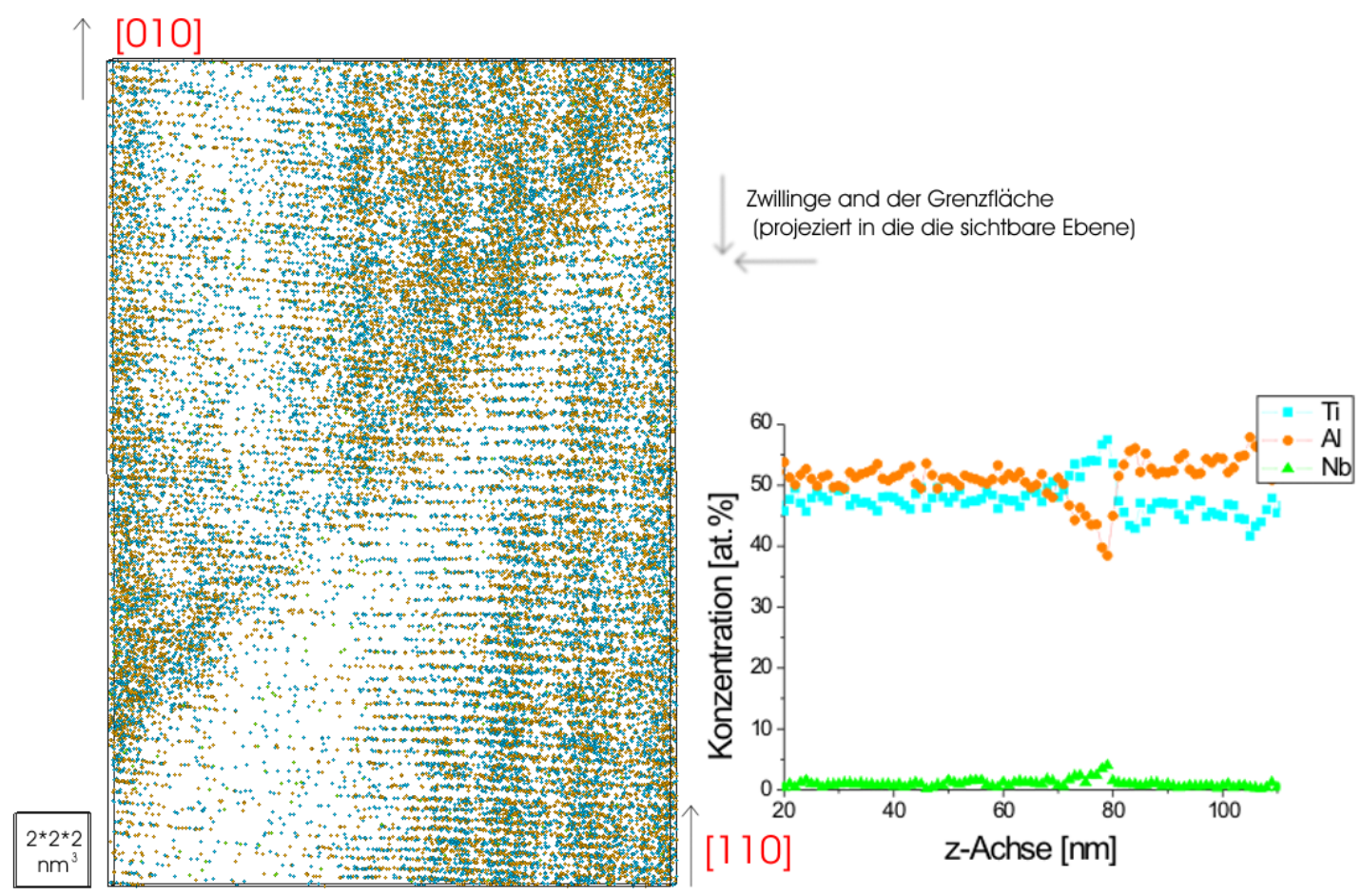

Abbildung 6.21: Eine [011]-Zwillingsgrenze in $\gamma-T i_{51} A l_{48} N b_{1}$ (links). Man kann erkennen, dass sich die Ebenenabstände vor und nach der Grenze unterscheiden. Dies ist darauf zurückzuführen, dass im unteren Bildteil eine [110]-Orientierung (in z-Richtung) vorhanden ist und nach der Grenze eine [010]-Orientierung. Die geringere Dichte im Bereich der [110]-Orientierung resultiert aus der höheren Feldverdampfungsfeldstärke in diese Richtung, welche einen lokalen Vergrößerungseffekt bewirkt. Das Konzentrationsprofil (rechts) zeigt an der Stelle der Domänengrenze (bei $z=80 \mathrm{~nm}$ ) eine Änderung der Konzentration und eine besonders hohe Nb-Konzentration. Die z-Achse verläuft von unten nach oben und geht über das links dargestellte Gebiet hinaus, um die leicht unterschiedliche Konzentration in den verschiedenen $\gamma$-Lamellen zu zeigen. 


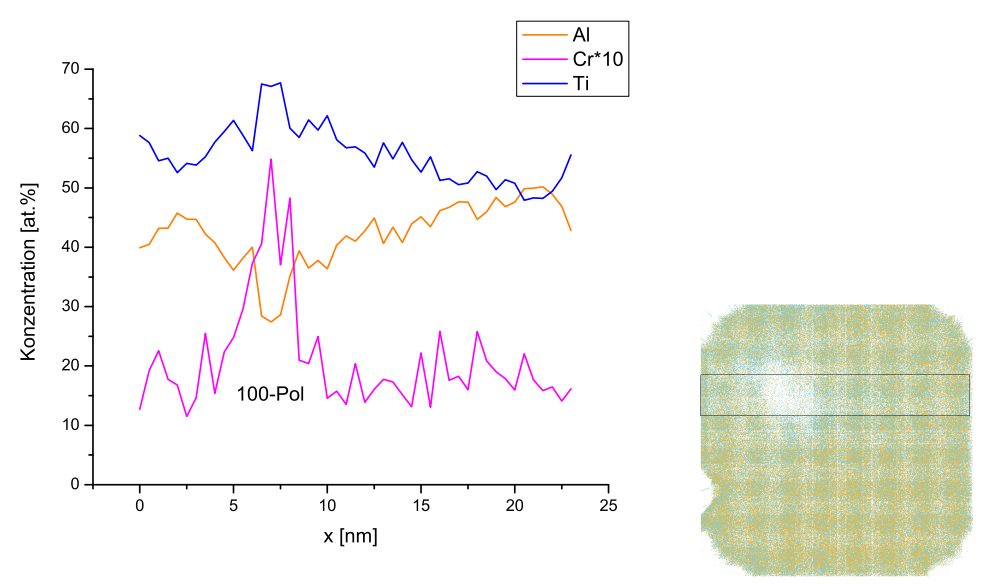

Abbildung 6.22: $\mathrm{Ti}_{52} \mathrm{Al}_{45} \mathrm{Cr}_{3}$, die Messrichtung ist [100].Abgebildet ist ein Konzentrationsprofil (links) des selektierten Bereichs (rechts) durch den Pol. Die Konzentration von Ti und Cr innerhalb des Pols ist erhöht. Die Gesamtfläche der Messung (rechts) ist 24x24 $n m^{2}$.

\subsection{Lokaler Vergrößerungseffekt}

Die Pole in FIM-Bilder lassen sich anhand der Ringe identifizieren. Auch im TAP können Pole erkannt werden. Sie äußern sich in Bereichen geringere Dichte, die durch den lokalen Vergrößerungseffekt entstehen. In Abb. 6.22 ist eine TAP-Messung mit (100)-Pol von $\mathrm{Ti}_{52} \mathrm{Al}_{45} \mathrm{Cr}_{3}$ abgebildet. Betrachtet man ein Konzentrationsprofil durch diesen Pol, so ist dort die Zusammensetzung verändert. Ursache sind unterschiedliche lokale Vergrößerungseffekte für die Materialien. Der lokale Vergrößerungseffekt muss also bei der Bestimmung der Konzentration berücksichtigt werden. Direkt an einem Pol liefert eine Konzentrationsmessung ein falsches Ergebnis. Deswegen wurden die häufig bei den TAP-Ergebnissen angegeben Konzentrationen über das gesamte gemessene Volumen bestimmt.

Für die geordneten Richtungen sollte man erwarten, dass das hinzulegierte Element sich immer so verhält wie das Element, dessen Plätze es belegt. Dafür ist allerdings keine ausschließliche, sondern nur eine bevorzugte Besetzung dieser Plätze notwendig. Generell verhalten sich alle untersuchten ternären Elemente (Ag, Cr, Nb) wie Ti. Somit besetzen sie eher Ti-Plätze. Eine quantitative Aussage ist nicht möglich.

Für $\mathrm{Cu}_{3} \mathrm{Au}$ beobachtet man für geordnete und nicht geordnete Richtungen einen jeweils anderen Vergrößerungseffekt für die Ionen (Abb. 6.23). An einem geordneten Pol ist eine höhere Dichte von $\mathrm{Cu}^{2+}$-Ionen $\mathrm{zu}$ finden. $\mathrm{Au}$ und $\mathrm{Cu}^{+}$sind dort ausgedünnt. Für nicht geordnete Richtungen beobachtet man eine leicht höhere Konzentration von Au um den Pol. Aus dem lokalen Vergrößerungseffekt lässt sich ein 


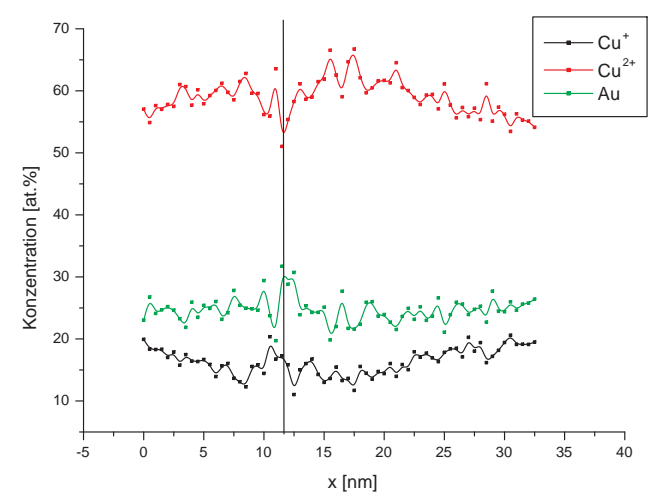

(a) (111)-Pol

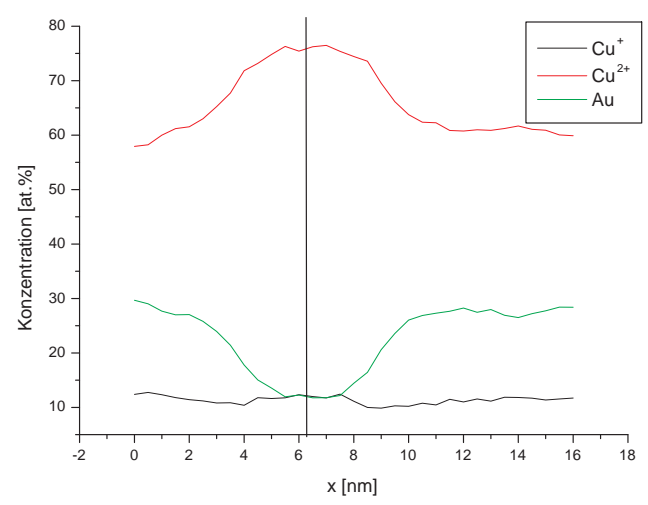

(b) (110)-Pol

Abbildung 6.23: Konzentrationsprofile um Pole in $C u_{3} A$. Das Verhalten an geordneten und nichtgeordneten Polen unterscheidet sich hier. Am nicht geordneten (111)-Pol (a) bemerkt man eine verringerte Dichte von $\mathrm{Cu}^{2+}$, wodurch die Konzentration von $\mathrm{Au}$ und der einfach geladenen Cu-Ionen erhöht wird. Am geordneten (110)-Pol (b) hingegen ist die Dichte von $\mathrm{Cu}^{2+}$ erhöht. An geordneten Polen ist der Effekt zudem erheblich stärker.

Hinweis auf die Feldverdampfungsfeldstärken der Materialien extrahieren. Die lokale Vergrößerung sollte umso größer sein, je weiter die Atome aus ihrer Umgebung hervorragen. Dabei kann man bei den nichtgeordneten Richtungen von einer stärkeren Vergrößerung für Elemente mit höherem $E_{\text {evap }}$ ausgehen. Für TiAl $+\mathrm{X}$ lässt sich somit feststellen, dass $\mathrm{Al}$ meistens eine höhere Feldverdampfungsfeldstärke besitzt als $\mathrm{Ti}$ (bei hohen Ti- oder Nb-Anteilen lässt sich oft ein anderer Zusammenhang beobachten). Für $\mathrm{Cu}_{3} \mathrm{Au}$ ist eine solche Feststellung nicht möglich. Die Feldverdampfungsfeldstärken für geordnete und nichtgeordnete Richtungen differieren hier aufgrund unterschiedlicher Ionisationsgrade.

\subsection{Verschmierung der Ebenen außerhalb der Pole}

Die Verschmierung der Ebenen außerhalb der Pole, wie sie z.B. in Abb. 6.19 zu erkennen ist, ist durch die veränderte Feldverdampfung in der Nähe der Pole und ihren Einfluss auf die Rekonstruktion bedingt. In FIM-Experimenten lässt sich gut erkennen, was geschieht. Die oberste Lage der Atome ist durch den innersten hellen Ring im FIM-Bild ( Abb. 6.7 D) sichtbar abgegrenzt. Nach einer anfänglich langsamen Verdampfung an dieser Stelle, die sich durch eine geringe Veränderung des Ringradius äußert, beschleunigt sich der Vorgang erheblich und der Ring schrumpft schnell (E) bis er schließlich verschwindet(F). In diesem Zeitraum sind außerhalb des Pols 
nur wenige Atome verdampft. Dadurch, dass die $z$-Position der Atome durch den Rekonstruktionsalgorithmus (Kap.3.1.3) aus der Verdampfungsreihenfolge ermittelt wird, ist die Streuung für die vom Pol abgelösten Atome geringer als für eine mehr zufällig bestimmte Verdampfung, wie sie abseits der Pole stattfindet. Dieser Effekt lässt sich mit den durchgeführten Simulationen (Kap. 7.6.4) reproduzieren.

\subsection{TAP: Feldverdampfungsfeldstärken}

Betrachtet man AtomVicinity-Dichteprofile von einfachen binären Legierungen, in der ein Atom des hinzulegierten Elements ein Element der Matrix substituiert, so würde man erwarten, dass die Peaks der verschiedenen Elemente deckungsgleich sind. Dies sollte unabhängig davon sein, in welche Richtung man misst. Aber das ist nicht der Fall. Die Ursache ist hier der Unterschied in den Feldverdampfungsfeldstärken. Um einen Pol herum verdampfen die Atome ebenenweise von außen nach innen. Dabei wird jeweils in jedem Spannungspuls das Atom abgelöst, dessen Feldverdampfungsfeldstärke von dem an ihm vorliegenden Feld übertroffen wird. Ist ein Atom $a$ mit geringer Feldverdampfungsfeldstärke von den umgebenden Atomen freigelegt, so wird es mit hoher Wahrscheinlichkeit innerhalb des nächsten Spannungspulses verdampfen. Innerhalb einer Legierung, in der das andere Element $b$ überwiegt, wird das vorhergehende Atom mit großer Wahrscheinlichkeit vom Typ $b$ gewesen sein. Da die z-Position-Atome durch die Reihenfolge bestimmt wird, liegt ein Atom mit der niedrigeren Feldverdampfungsfeldstärke hinter dem mit der höheren. Im Fall einer höheren Feldverdampfungsfeldstärke von $a$ widersteht es der Evaporation länger. Sobald es jedoch feldverdampft, ist das Feld für die, zuvor von ihm festgehaltenen, Atome $b$ hoch genug, dass diese ebenfalls verdampfen. Die AtomVicinity-Peaks des Atoms mit der höheren Feldverdampfungsfeldstärke liegen nun vor denen von $b$.

Im Grunde bestimmt man dadurch natürlich nur die Verhältnisse für die gemessene Richtung. Da sich die Feldverdampfungsfeldstärken für reine Materialien jedoch nur geringfügig in Abhängigkeit der Richtung ändern (maximal 3\% wurden in dieser Arbeit für W in Kap. 6.4.4.1 bestimmt), kann man davon ausgehen, dass dies auch für die einzelnen Elemente in einer Legierung zutrifft. Die massivere Änderung von $E_{\text {Evap }}$ in $\gamma$-TiAl (Kap. 6.4.5) liegt an der Existenz von Überstrukturrichtungen und sollte sich nicht auf die Verhältnisse der Elemente untereinander auswirken. 


\subsubsection{Ein einfaches binäres Systeme: NiAl}

Es wurde Ni8at.\%Al bei $40 \mathrm{~K}$ und einer Pulsspannung von 15\% der Basisspannung gemessen. Die nominelle Konzentrationen stimmt mit der im TAP gemessenen von Ni8.7(1)at.\%Al einigermaßen überein, wenn man berücksichtigt, dass die Konzentration in verschiedenen Körnern abweichen kann. Nach den Phasendiagrammen kann man erwarten, dass die Ni-Phase vorliegt. Der (002)-Pol, um den herum das mit AtomVicinity zu analysierende Volumen ausgewählt wurde, lag innerhalb der Messung. Zur Bestimmung der Dichteprofile wurden die Ebenen senkrecht zur $z$-Achse ausgerichtet. Die sich ergebenden AtomVicinity-Dichteprofile sind in Abb. $6.24 \mathrm{zu}$ sehen. Die Ebenenabstände wurden durch Anpassung des Parameter $E \beta$ auf die erwarteten Werte von (002)-kfz-Nickel (0.176 nm) eingestellt.

Die Auswertung erbringt eine nachvollziehbare Abschätzung der Feldverdampfungsfeldstärken mit $E_{E v a p, N i}>E_{E v a p, A l}$, die mit den nach Müller-Schottky berechneten Literaturwerten der reinen Materialien $E_{E v a p, N i}=35 \mathrm{~V} / \mathrm{nm}$ und $E_{E v a p, A l}=33 \mathrm{~V} / \mathrm{nm}$ [Mil11996] übereinstimmt. Die Methode wurde auch auf Al8at.\%Li angewandt. Da dieses System jedoch keine einfache kfz-Matrix mit homogener Atomverteilung mehr besitzt, befinden sich die Ergebnisse hierzu im Anhang B.

Dass im Falle einer höheren Feldverdampfungsfeldstärke das zulegierte Element auch vor dem Hauptpeak auftauchen kann, wird später im folgenden Abschnitt anhand von $\mathrm{Nb}$ in TiAl gezeigt.

\subsubsection{Abschätzung der Feldverdampfungsfeldstärken in Ti- $\mathrm{Al}+\mathrm{X}$}

\subsubsection{1 $\mathrm{TiAl}+\mathrm{Nb}$}

Betrachtet man AtomVicinity-Dichteprofile von TiAl+Nb für nichtgeordnete Richtungen, so erhält man in allen Fällen die Peakabfolge Ti-Al-Nb (Abb. 6.25). Die Feldverdampfungsfeldstärke von Nb ist somit am höchsten, die von Ti am geringsten. Da Al in den Messungen praktisch ausschließlich 2-fach ionisiert auftaucht, ist dies durchaus plausibel. Für einfach ionisiertes Al wäre (nach [Mill1996]) mit $E_{\text {Evap }, A l}=19 \mathrm{~V} / \mathrm{nm}$ ein geringerer Wert als für Ti (das zweifach geladen auftritt) mit $26 \mathrm{~V} / \mathrm{nm}$ zu erwarten. Da es zweifach geladen auftritt, sind $35 \mathrm{~V} / \mathrm{nm}$ für Al plausibel und erklären die höhere Feldverdampfungsfeldstärke. Für Nb wird mit $37 \mathrm{~V} / \mathrm{nm}$ in der Tat die höchste Feldverdampfungsfeldstärke erwartet. 


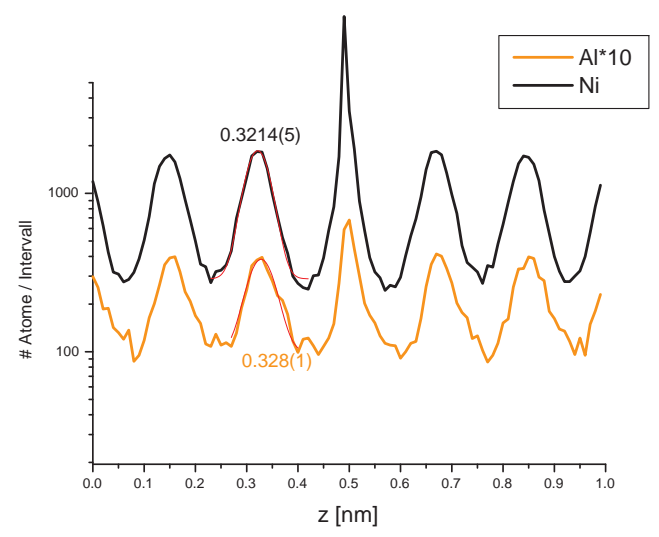

(a) Ni8at.\%Al: Referenzatom $=\mathrm{Ni}$

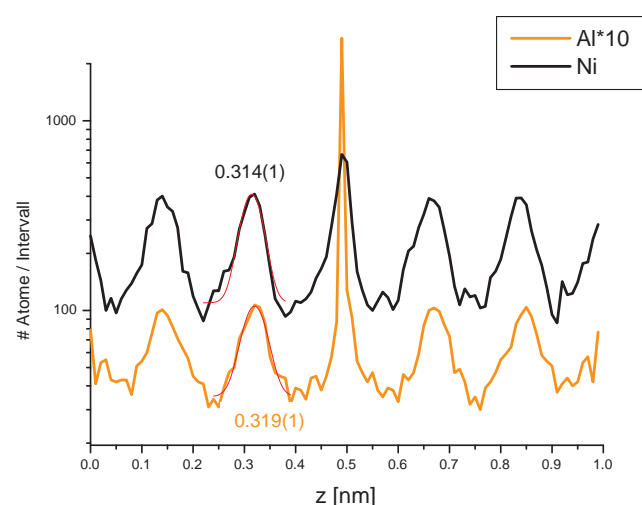

(b) Ni8at.\%Al: Referenzatom $=\mathrm{Al}$

Abbildung 6.24: AtomVicinity-Dichteprofile von Ni8at.\%Al. Man sieht, dass sich, unabhängig davon, welches Element als Referenzatom definiert wird, eine Peakreihenfolge erkennbar ist. Die an den Peaks notierten Werte sind die z-Positionen aus dem Gaussfit. Sowohl in (a) als auch in (b) ist die Reihenfolge Ni-Al.

\subsubsection{2 $\quad \mathrm{Ti}_{46} \mathrm{Al}_{52} \mathrm{Ag}_{2}$}

Für $\mathrm{Ti}_{46} \mathrm{Al}_{52} \mathrm{Ag}_{2}$ ist eine Bestimmung der Verhältnisse von $E_{\text {Evap }}$ anhand der AtomVicinity-Dichteprofile in [100]-Richtung nicht direkt möglich. Ein entsprechendes Dichteprofil für die [100]-Richtung wurde bereits in Kap. 4.3 in Abb. 4.5 gezeigt. Durch die Kreuzkorrelation lässt sich eine Peak-Reihenfolge Ti-Ag-Al ausmachen. Dies bedeutet, dass $E_{E v a p, A g}$ zwischen $E_{E v a p, T i}$ und $E_{E v a p, A l}$ liegt. Anhand der Literaturdaten [Mill1996] wäre für $\mathrm{Ag}^{+}$mit $E_{E v a p}=24 \mathrm{~V} / \mathrm{nm}$ ein, im Vergleich zu Ti mit $26 \mathrm{~V} / \mathrm{nm}$, niedrigerer Wert zu erwarten gewesen. Da Ag jedoch bei unseren Messungen teilweise auch zweifach geladen detektiert wurde, könnte man auch $45 \mathrm{~V} / \mathrm{nm}$ vermuten.

Aus der vorliegenden $\mathrm{L} 1_{0}$-Phase wandert Ag, wie die sich in TEM-Analysen in Kap. 6.1.2 zeigende Entmischung andeutet, herauswandert, sollte die Feldverdampfungsfeldstärke eher niedriger sein als in reinem Ag.

\subsubsection{3 $\mathrm{Ti}_{52} \mathrm{Al}_{45} \mathrm{Cr}_{3}$}

Auch für $\mathrm{Ti}_{52} \mathrm{Al}_{45} \mathrm{Cr}_{3}$ zeigen Messungen in [100]-Richtung in AtomVicinity-Dichteprofilen von Messungen mit ähnlich großen Anteilen von $\mathrm{Cr}$ und $\mathrm{Ti}$ ein starkes Rauschen, weshalb hier ebenfalls eine Kreuzkorrelation notwendig ist. Hier ist ein statistisch gerade noch signifikanter Positionsunterschied feststellbar (Abb. 6.26). 


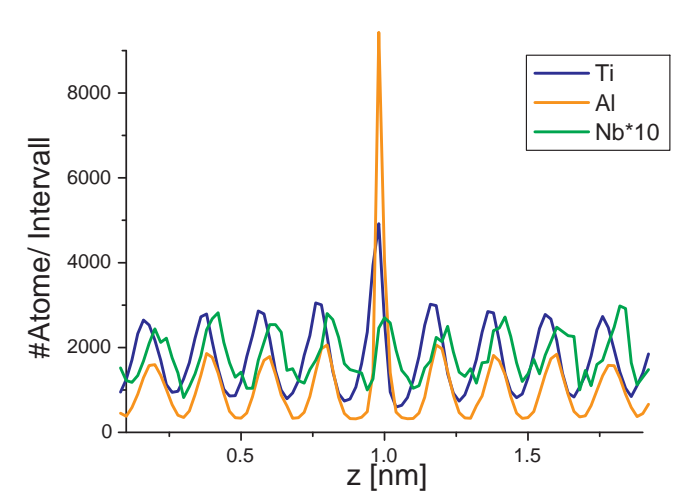

(a) $\mathrm{Ti}_{51} \mathrm{Al}_{48} \mathrm{Nb}_{1}$

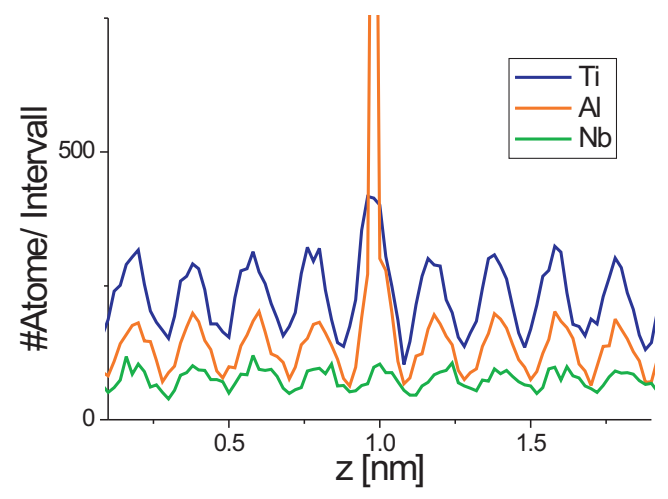

(b) $\mathrm{Ti}_{47} \mathrm{Al}_{48} \mathrm{Nb}_{5}$

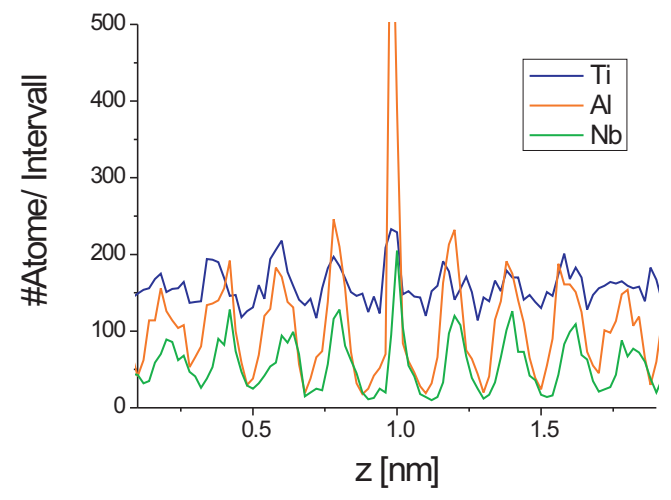

(c) $\mathrm{Ti}_{42} \mathrm{Al}_{48} \mathrm{Nb}_{10}$

Abbildung 6.25: Atom Vicinity-Dichteprofile in die nichtgeordnete [100]-Richtung (Referenzatom: Al) von $\gamma$-TiAl für verschiedenen Nb-Konzentrationen. Al ist das Referenzatom. Die Peakreihenfolge ist immer Ti-Al-Nb [Boll2005]. 

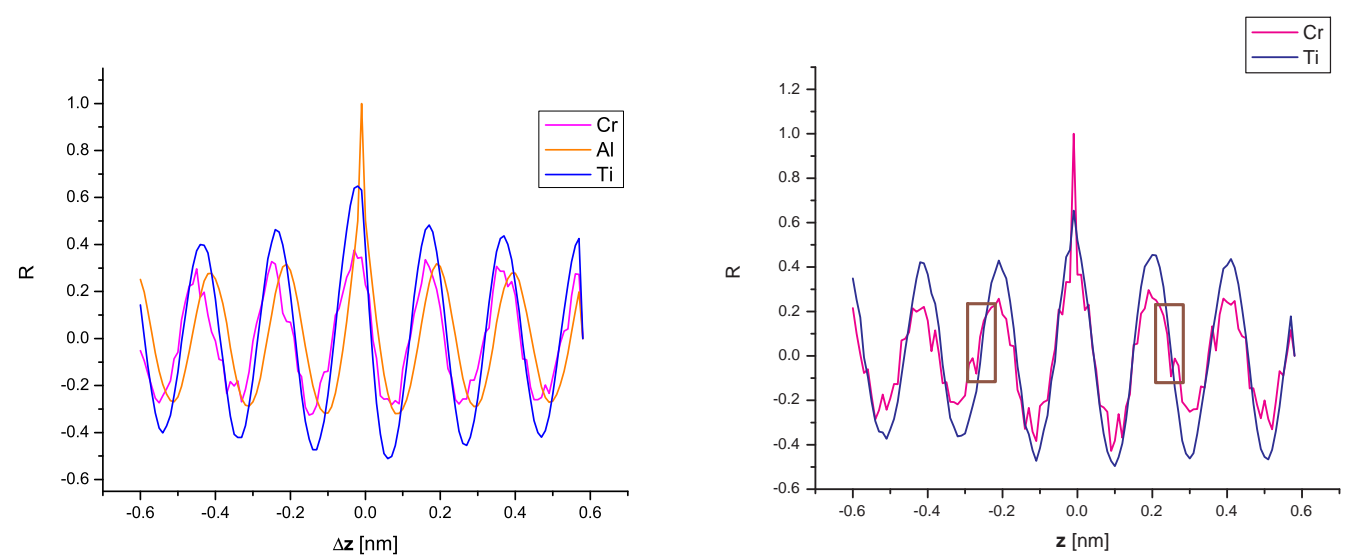

Abbildung 6.26: AtomVicinity-Ergebnisse für Ti48.5at.\%Al1.6at.\%Cr mit Al als Referenzatom. Die Kreuzkorrelationen mit Al (links) zeigen, dass Al das höchste $E_{\text {Evap besitzt. }}$ Die Unterscheidung von $\mathrm{Cr}$ und Ti hingegen ist schwierig. Die Kreuzkorrelation von Ti mit $\mathrm{Cr}$ (rechts) erlaubt eine Aussage über die Reihenfolge. Die Verschiebung beträgt-0.009(1) nm. Gut lässt sich dies erkennen, wenn man sich veranschaulicht, dass die Cr-Peaks um den mittleren Peak gespiegelt sind. Nun kann man die Überlappung der Ti- mit den CrPeaks betrachten. Die Ti-Peaks sind offenbar, wie man etwa beim Vergleich der markierten Rechtecke erkennt, leicht nach links verschoben. Die Verschiebung ließ sich mit Gaussfits bestimmen.

Der Trend geht zu einer Position links von Ti, was ein geringeres $E_{\text {Evap }}$ bedeuten würde. Mit $27 \mathrm{~V} / \mathrm{nm}$ für $\mathrm{Cr}^{+}$und $29 \mathrm{~V} / \mathrm{nm}$ für $\mathrm{Cr}^{2+}$ [Mill1996] liegen die erwarteten Feldverdampfungsfeldstärken im Bereich von Ti. Da sich Cr gut in der betrachteten Phase lösen lässt, ist es sinnvoll, dass auch hier die Feldverdampfungsfeldstärke vergleichbar mit Titan ist.

Die Reihenfolge lässt sich am besten bestimmen, wenn man die Kreuzkorrelation von $\mathrm{Cr}$ mit Ti betrachtet. Damit ergibt sich für die Feldverdampfungsfeldstärken $E_{E v a p, C r}<E_{E v a p, T i}<E_{E v a p, A l}$. Die äußerst geringe Verschiebung der Cr- zu den Ti-Peaks, die sich in fast allen Messungen statistisch nicht signifikant war, lässt allerdings die Annahme, dass sich das $E_{E v a p, C r}$ der geordneten Richtungen genauso verhält, riskanter erscheinen als für $\mathrm{Ag}$ oder $\mathrm{Nb}$. Deswegen sollte die Aussage vorsichtshalber auf $E_{\text {Evap,Cr }} \leq E_{E v a p, T i}<E_{\text {Evap,Al }}$ beschränkt werden.

In einer Messung, in der der Ti-Anteil gegenüber dem Al-Anteil stark überwiegt (Ti43.8at.\%Al2.8at.\%Cr), beobachtet man, wie bei den Nb-haltigen Proben, ein eindeutiges Verhalten. Die Peakreihenfolge ist Ti-Cr-Al. Erklären lässt sich dies durch zwei unterschiedliche Cr-Bindungen auf Ti oder auf Al-Plätzen, wie später durch Simulationen in Kap.7.6.5 gezeigt wird. 


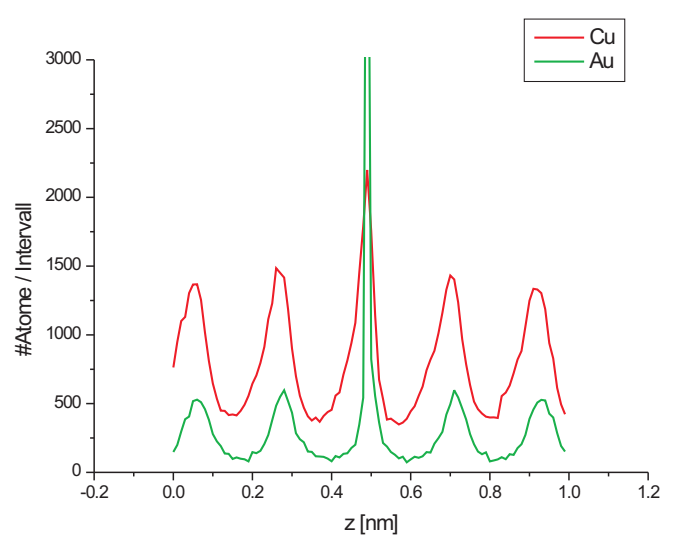

Abbildung 6.27: AtomVicinity-Dichteprofil von 75.0at.\%Cu25.0at.\%Au am (111)-Pol mit Au als Referenzatom. Es werden gemischt besetzte Ebenen erwartet und beobachtet. Die AuPeaks sind um 0.026(2) nm nach rechts verschoben.

\subsection{2 $\mathrm{Cu}_{3} \mathrm{Au}$}

Die niedrigst indizierte nichtgeordnete Richtung in $\mathrm{Cu}_{3} \mathrm{Au}$ ist die $<111>$-Richtung. Man erhält in Abb. 6.27 ein klare Ebenenreihenfolge von $\mathrm{Cu}-\mathrm{Au}$, so dass $E_{\text {Evap,Cu}}>$ $E_{E v a p, A u}$ gilt. Vergleicht man dies mit den Literaturwerten für die Feldverdampfungsfeldstärken, so ist dies ein unerwartetes Ergebnis. Nach [Mil11996] würde man rechnerisch und nach experimentellen Ergebnissen $E_{\text {Evap }, C u^{+}}=30 \mathrm{~V} / \mathrm{nm}$ erwarten. Selbst die Feldverdampfungsfeldstärke $E_{E v a p, C u^{2+}}=43 \mathrm{~V} / \mathrm{nm}$ von zweifach geladenem $\mathrm{Cu}$ wäre somit immer noch im Bereich der experimentell ermittelten Feldverdampfungsfeldstärke von $\mathrm{Au}$ mit $E_{\text {Evap,Au,gemessen }}=35 \mathrm{~V} / \mathrm{nm}$ und weit unter der berechneten mit $E_{E v a p, A u^{2+}}=54 \mathrm{~V} / \mathrm{nm}\left(E_{E v a p, A u^{+}}=53 \mathrm{~V} / \mathrm{nm}\right)$. Zudem ist aus den Massenspektren (Kap. 6.4.3) bekannt, dass $\mathrm{Cu}$ zum großen Teil einfach ionisiert auftritt. In Kap. 7.5.2 wird sich dieses ungewöhnliche Ergebnis allerdings durch eine Simulation reproduzieren lassen. Da in anderen Richtungen die Feldverdampfung mit einem größeren Anteil zweifach geladener Cu-Ionen stattfinden kann, muss dort auch die Feldverdampfungsfeldstärke von $\mathrm{Cu}$ abweichen.

Allerdings erwartet man rechnerisch für zweifache $\mathrm{Cu}$-Ionen eine noch höhere Feldverdampfungsfeldstärke als für einfach geladene, so dass man davon ausgehen kann, dass die hier ermittelte Beziehung auch dort gültig ist. 


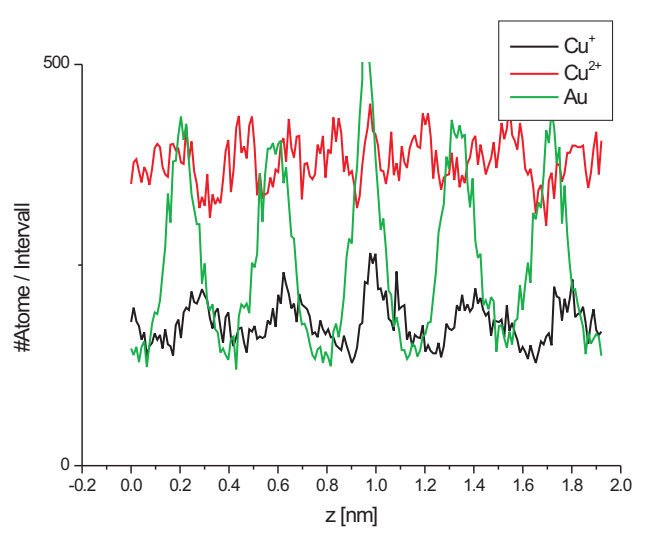

(a) Dichteprofile

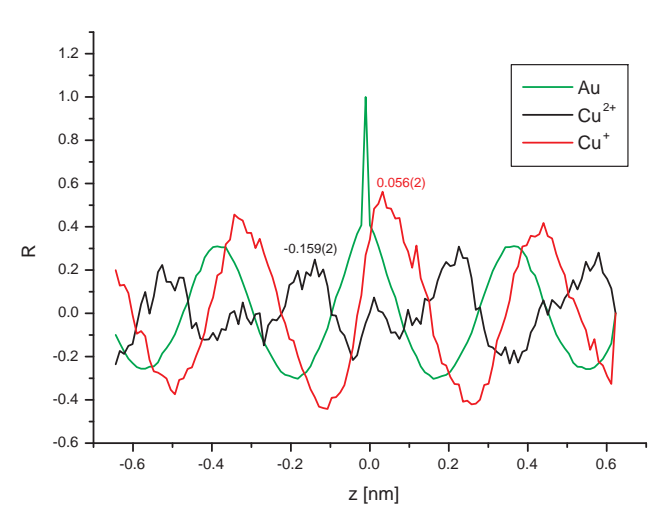

(b) Kreuzkorrelationen mit $\mathrm{Au}$

Abbildung 6.28: AtomVicinity-Dichteprofile von 70.9at.\%Cu29.1at.\%Au mit Au als Referenzatom für die $<100>-$ Richtung (a). Offenbar bedingen die unterschiedlich geladenen Cu-Ionen unterschiedliche Peaks. Dies wird besonders deutlich, wenn man Kreuzkorrelationen $R$ der Daten (b) aus (a) mit Au (aus (a)) betrachtet. Es lassen sich mit diesem Verfahren recht genaue Peakpositionen angeben.

\section{9 $\mathrm{Cu}_{3} \mathrm{Au}$ in Überstrukturrichtungen}

Die am niedrigsten indizierte Überstrukturrichtung in $\mathrm{Cu}_{3} \mathrm{Au}$ ist die $<100>$-Richtung. Es wechselt sich dabei eine reine $\mathrm{Cu}$-Ebene mit einer gemischten $\mathrm{Cu} / \mathrm{Au}$-Ebene ab. Da Cu nach Kap. 6.8.2 eine höhere Feldverdampfungsfeldstärke hat als $\mathrm{Au}$, ist zu erwarten, dass in den AtomVicinity-Dichteprofilen nach einem kleinen Cu-Peak unmittelbar ein Au-Peak und im Abstand der halben Gitterkonstante ein reiner CuPeak auftreten. Da die Qualität der Messungen in diese Richtung sehr schlecht war, lässt sich in einem gewöhnlichen AtomVicinity-Dichteprofil eine derartige Ebenenaufsplittung nicht beobachten. Betrachtet man die $\mathrm{Cu}^{+}$und $\mathrm{Cu}^{2+}$-Ionen unabhängig voneinander (Abb. 6.28a), wird klar, dass die weniger zahlreichen $\mathrm{Cu}^{2+}$-Ionen die erwartete Peakaufspaltung liefern können. Da die Daten jedoch sehr verrauscht sind, bietet es sich an, Kreuzkorrelationen mit den Au-Peaks zu betrachten (Abb. 6.28b). Dadurch lassen sich die Peakpositionen ziemlich genau ablesen. Dabei ist insbesondere die Distanz des zweifach geladenen Cu-Peaks zum Goldpeak mit 0.159(2) nm von Interesse. Der Abstand zum Peak der zweifach geladenen Cu-Ionen ist wesentlich größer als der zum Peak der einfach geladenen Ionen. 


\subsection{Bestimmung der Platzbesetzung in $\mathrm{TiAl}+\mathrm{X}$}

Der Titel dieses Unterkapitels könnte auch "Messungen in Überstrukturrichtungen von TiAl $+\mathrm{X}$ " lauten. Das Interessante an diesen Messungen ist, dass sie verwendet werden können, um eine Platzbesetzung der hinzulegierten Elemente zu bestimmen. Dafür ist es wichtig, das Verdampfungsverhalten zu kennen. Besitzt das Element eine niedrigere Feldverdampfungsfeldstärke als das Element, in dessen Ebene es sitzt, so wird es tendenziell vor diesem Element verdampfen. Umgekehrt wird es, falls es eine höhere Feldverdampfungsfeldstärke besitzt, eher nach dem Element verdampfen, in dessen Ebene es sitzt. Hierbei helfen die Ergebnisse für die Feldverdampfungsfeldstärken, die aus den Messungen für die nichtgeordneten Richtungen gewonnen werden konnten. Da sich aber die Platzbesetzung auch für diese Richtungen wichtig erweisen kann, ist eine Vorhersage des Verdampfungsverhaltens für die geordneten Richtungen gewagt. Es ist deswegen sinnvoll auch die Simulationen (Kap. 7.6.6) heranzuziehen, anhand derer die AtomVicinity-Peakreihenfolgen für verschiedene Platzbesetzungszenarien vorhergesagt werden können.

Ein anderes Argument für die Platzbesetzung kann die Peakform sein, die, zumindest, falls das zulegierte Element eine geringere oder vergleichbar große Feldverdampfungsfeldstärke besitzt, dem Element ähneln sollte, dessen Plätze es besetzt.

Sind die Ti- und Al-Peaks gut unterscheidbar, so könnte man annehmen, dass die Berücksichtigung der Feldverdampfungseigenschaften nicht mehr von Bedeutung ist. Die Peakposition des hinzulegierten Elements sollte, ähnlich wie es bei den nichtgeordneten Richtungen, nicht stark von der Peakposition des substituierten Elements abweichen. Dies ist allerdings nicht unbedingt gegeben. Es kann durchaus sein, dass in einer gemischten Ebene zuerst alle Elemente mit niedriger Feldverdampfungsfeldstärke verdampfen, bevor das Element mit der höheren Feldverdampfungsfeldstärke abgetragen wird.

Falls eine Besetzung verschiedener Plätze vorliegt, kann man eine quantitative Angabe der Platzbesetzung vornehmen. Hierfür vergleicht man die dem jeweiligen MatrixElement zugeordneten Flächen unter den AtomVicinity-Dicheprofilpeaks (abzüglich des Rauschanteils) des hinzulegierten Elements. Aus Gaussfits können Angaben über den Fehler ebenso erfolgen, wie über den Vergleich mehrerer Peakkombinationen. Es ist jeweils der größere der beiden Fehler angegeben.

Aufgrund des lokalen Vergrößerungseffekts weicht die Konzentration im Analysebereich und in den AtomVicinity-Dichteprofilen von der tatsächlich vorliegenden 
Konzentration, welche durch Einbeziehen des Bereichs um den Pol herum ermittelt werden kann, ab. Es ist nun zu überlegen, inwiefern das hinzulegierte Element diesem Vergrößerungseffekt und der daraus resultierenden Dichteschwankung unterliegt. Die naheliegendste Vermutung wäre, anzunehmen, dass sich das hinzulegierte Element so verhält, wie das Element, dessen Plätze es substituiert. Allerdings kann für das zulegierte Element in fast allen Fällen eine große Abweichung der Peakposition zu den Peakposition des substituierten Elements festgestellt werden. Diese Abweichung ist meist größer als die Abstände der Ti- und Al-Peaks. Lediglich die Ag-haltigen Proben weichen von diesem Schema ab.

Es ist somit nicht sinnvoll, die berechneten Platzbesetzungen durch einen aus dem Vergleich der realen und im Analysebereich vorliegenden Konzentrationsverhältnisse von $\mathrm{Ti}$ und $\mathrm{Al}$ bestimmten Korrekturfaktor zu berichtigen. Stattdessen wird davon ausgegangen, dass der lokale Vergrößerungseffekt und somit die Dichteabweichung für beide Platzbesetzungen identisch ist. Diese Näherung ist nicht unbedeutend. Die Konzentration von Al kann im AtomVicinity-Dichteprofil um bis zu 100\% höher als in der Umgebung sein.

\subsubsection{Platzbesetzung von $\mathrm{Nb}$}

Für TiAl+Nb konnte, wie Abb. 6.29 zeigt, in allen Fällen für die [110]- und [001]Überstrukturrichtungen die Peakreihenfolge Al-Ti-Nb beobachtet werden. $\mathrm{Da} \mathrm{Nb}$ zumindest eine höhere Feldverdampfungsfeldstärke als Ti besitzt, liegt es somit überwiegend auf Ti-Plätzen. Würde es auf Al-Plätzen liegen, so müsste, falls es auch absolut die höchste Feldverdampfungsfeldstärke besitzt, sein Peak zwischen dem Ti- und Al-Peak liegen. Besitzt es eine niedriger Feldverdampfungsfeldstärke als Al, so müsste es vor den beiden anderen Peaks auftauchen.

Man könnte argumentieren, dass Nb beide Plätze besetzt, dies aber nicht beobachtet werden kann, da die Peaks dicht beieinander liegen. Die Betrachtung einiger Messungen, bei denen ein geringerer Ordnungsgrad vorlag und somit die Unterscheidung von Ti- und Al-Peaks möglich ist, zeigt, dass der Nb-Peak sich praktisch nie aufsplittet [Boll2005]. Die Aussage, dass $\mathrm{Nb}$ in $\mathrm{Ti}_{51} \mathrm{Al}_{48} \mathrm{Nb}_{1}$ undTi ${ }_{47} \mathrm{Al}_{48} \mathrm{Nb}_{5}$ fast ausschließlich Ti-Plätze belegt, ist somit gerechtfertigt. Anhand der Ergebnisse der Simulationen in Kap. 7.6.6 wäre bei einer Platzbesetzung der Al-Plätze eine Aufspaltung des Peaks zu erwarten. 


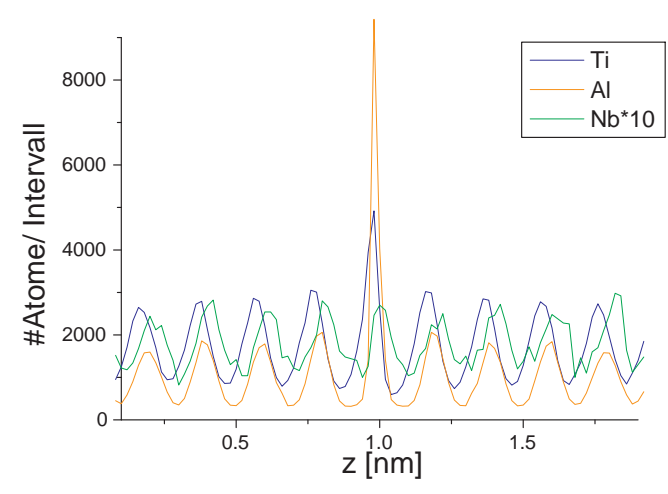

(a) $\mathrm{Ti}_{51} \mathrm{Al}_{48} \mathrm{Nb}_{1}$

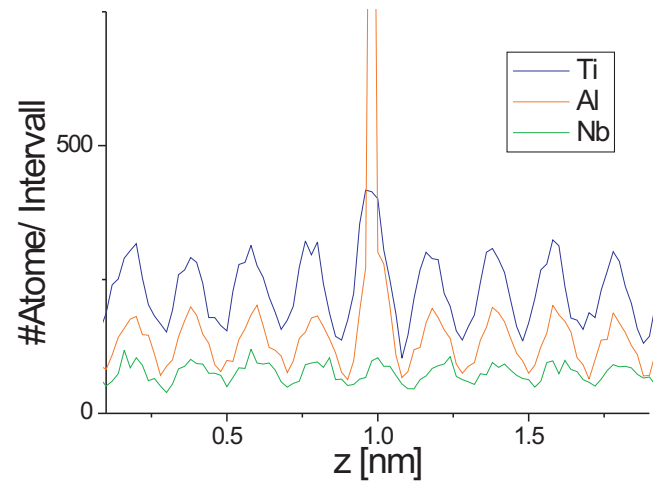

(b) $\mathrm{Ti}_{47} \mathrm{Al}_{48} \mathrm{Nb}_{5}$

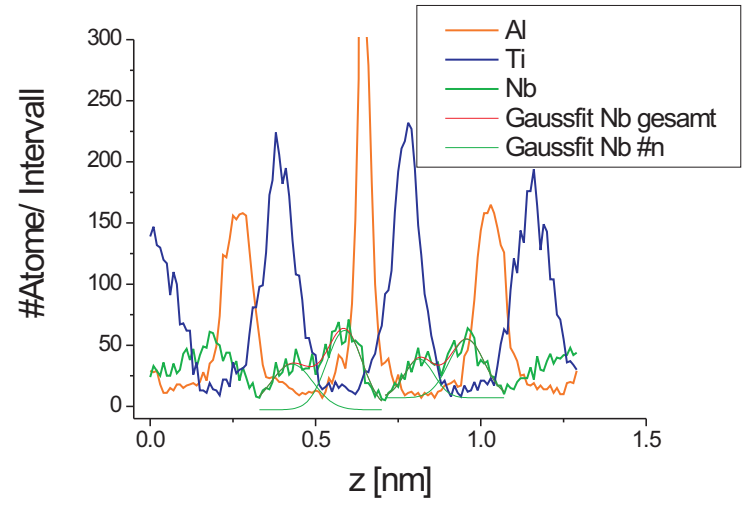

(c) Ti48.6at.\% Al8.7at.\% $\mathrm{Nb}$

Abbildung 6.29: AtomVicinity-Dichteprofile für TiAl+ $\mathrm{Nb}$ in die geordnete [001]Richtung (Referenzatom:Al). Für die 1at.\% und 5at.\% Nb enthaltenden Proben (a,b) lässt sich eine nahezu ausschließliche Präferenz für die Ti-Plätze feststellen. Auch leicht varriierende Konzentrationen ändern nichts an der Peakreihenfolge, weswegen die beiden Legierungen durch Repräsentanten dargestellt sind. Bei der 10 at.\% Nb (c) hingegen splittet sich der Peak auf. Mit Gaussfits lassen sich die Flächen unter den Peaks bestimmen. Hier ist die genaue, mit der Atomsonde bestimmte Konzentration angegeben. 
Bei Proben mit 10at.\% Nb war die Ebenenunterscheidung gut möglich. Hier kann allerdings eine Peakaufsplittung beobachtet werden. Mithilfe von Gaussfits kann man die Fläche unter den einzelnen Peaks ermitteln (abzüglich des Rauschsignals) und dadurch eine Nb-Platzbesetzung der Al-Plätze von 0.3(1) berechnen.

In den Dichteprofilen fällt auf, dass alle Nb-Peaks hinter den Ti-Peaks liegen. Die Nb-Atome der Al-Schicht müssten, wenn man davon ausgeht, dass Nb am schwersten feldverdampft, also erst nach den Atomen der darunter liegenden Ti-Schicht verdampfen. Dies erscheint wenig plausibel. Besser lässt sich die Peak-Aufspaltung durch die Existenz zweier verschiedener Bindungstypen für Nb erklären. Einerseits existieren Nb-Atome, die vor allem von Ti-Atomen umgeben sind, anderseits NbAtome, die viele Nb-Nachbarn haben und dadurch wesentlich stärker gebunden sind. Als dritte Möglichkeit muss in Betracht gezogen werden, dass Nb auf Al-Plätzen eine geringere Feldverdampfungsfeldstärke besitzt und deswegen vor dem in der gleichen Ebene befindlichen $\mathrm{Al}$ verdampft. In Kap. 7.6.6 wird gezeigt, dass sich in der Tat die Feldverdampfungsfeldstärke von Nb für die nichtgeordnete [100]-Richtung am höchsten sein kann und gleichzeitig in der [001]-Richtung geringer als die von Al.

Veröffentlichungen, die ebenfalls die Platzbesetung von $\mathrm{Nb}$ in $\gamma$-TiAl untersuchen, sagen alle eine starke Präferenz von Nb für die Ti-Plätze voraus. Auch in Fällen, in der die Ti-Konzentration weiter unter dem stöchiometrischen Gleichgewicht liegt [Wolf1996, Wood1998, NI2001, Hao1999]. Auch ALCHEMI-Untersuchungen ergeben eine Bevorzugung der Ti-Plätze [Koni1986, Hao2000, Hao1999, Moha1991, Ross1996]. Da Ti in unseren Proben immer weniger als 50at.\% der Legierung ausmacht, ist eine vernachlässigbare Besetzung der Ti-Plätze plausibel. Im Fall von $\mathrm{Ti}_{42} \mathrm{Al}_{48} \mathrm{Nb}_{10}$ ist eine $30 \%$ Besetzung von Al-Plätzen noch innerhalb des erwarteten Bereichs.

\subsubsection{Platzbesetzung von Ag}

Für $\mathrm{Ti}_{46} \mathrm{Al}_{52} \mathrm{Ag}_{2}$ erhält man eine Besetzung beider Plätze. Da die Feldverdampfungsfeldstärke von Ag zwischen der von Al und Ti liegt, sollten die Ag-Peaks in AtomVicinity-Dichteprofilen der Überstrukturrichtungen vor dem zugehörigen Alund hinter dem zugehörigen Ti-Peak erscheinen. In Abb. 6.30 kann man durch Ver-

gleich der Flächen unter den Peaks eine Platzbesetzung ausmachen. Dabei liegt der den AL-Plätzen zugeordnete Ag-Peak gleichauf bzw sogar leicht hinter dem Al-Peak. 


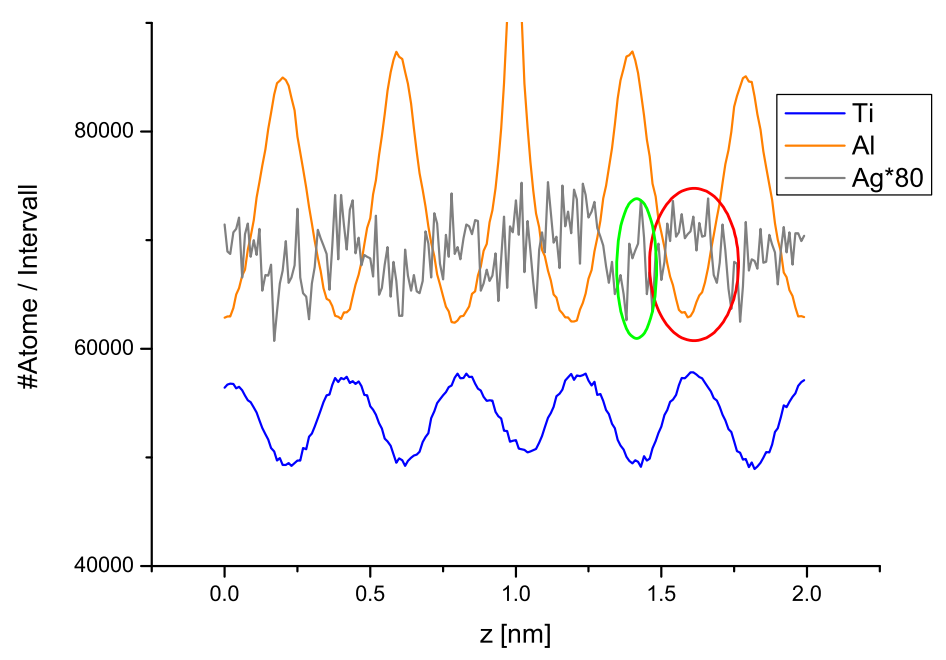

Abbildung 6.30: AtomVicinity-Dichteprofil der [001]-Richtung von Ti55.6at.\%Al0.7at.\%Ag mit Al als Referenzatom. Durch Vergleich der Flächen unter den durch die rote und grüne Ellipse markierten Peaks lässt sich die Platzbesetzung der Ag-Atome ermitteln. Die zu Al-Plätzen gehörenden Ag-Atome sind grün, die zu Ti gehörenden Ag-Atome rot umrandet. Demnach belegen 20\% der Ag-Atome Al-Plätze.

Dies kann dadurch erklärt werden, dass Ag auf Al-Plätzen vor allem zweifach ionisiert verdampft und somit eine weit höhere Feldverdampfungsfeldstärke besitzt als das einfach ionisierte Ag auf den Ti-Plätzen (45 V/nm anstelle von $24 \mathrm{~V} / \mathrm{nm}$ ). Die Statistik ist relativ schlecht. Die Ag-Konzentration liegt in allen Proben weit unter dem nominellen Wert (0.7at.\% war die höchste beobachtete Ag-Konzentration). Es gibt vermutlich andere Bereiche mit Clustern, in denen hohe Ag-Konzentrationen vorliegen, die nach Auslagerung der Proben als Ausscheidungen sichtbar werden (Kap. 6.1.2). Die Konzentration von Ag schwankte im Analysebereich meist stark, was als Hinweis auf beginnende Clusterbildung gewertet werden kann ${ }^{7}$. Die Platzbesetzung befindet sich also im Wesentlichen im Bereich dieser beginnenden Ausscheidungen einer AlAg-Phase. Es macht also durchaus Sinn, dass vor allem die Ti-Plätze substituiert werden. Für Ti55.6at.\%Al0.7at.\%Ag ergibt sich eine Platzbesetzung der Al-Plätze von 0.2(1). Für Ti56.2at.\%Al0.4at.\%Ag ergibt sich eine Ag-Besetzung der Al Plätze von 0.2(5).

Nach Yang et al. [Yang2000] ist eine Besetzung beider Plätze zu erwarten. Für Ti46at.\%Al3at.\%Ag ermittelte er experimentell eine Platzbesetzung der Al-Plätze von $80 \%$. Da hier Aluminium unterstöchiometrisch vorliegt, stimmt dies gut mit dem Ergebnis dieser Arbeit überein.

\footnotetext{
${ }^{7}$ für eine eindeutige Festlegung ist die Statistik zu schlecht
} 


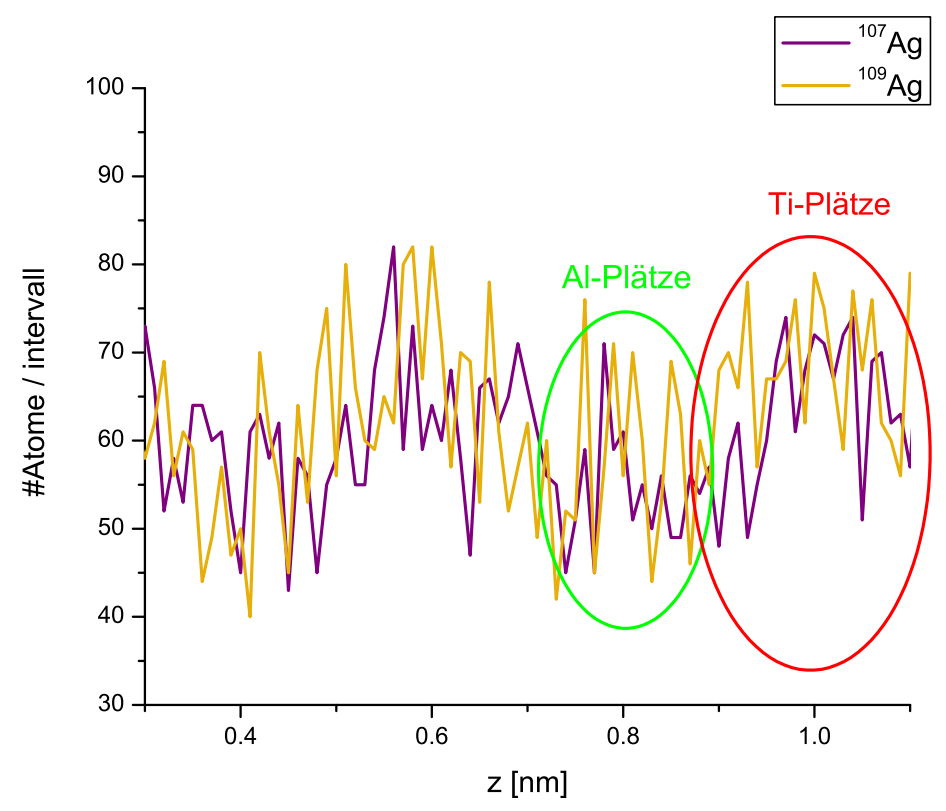

Abbildung 6.31: AtomVicinity-Dichteprofile der [001]-Richtung von Ti55.6at.\%Al0.7at.\%Ag für verschiedene Ag-Isotope mit Al als Referenzatom. Die Kurven haben einen ähnlichen Verlauf, was auf identische Platzbesetzung hindeutet. Aufgrund der geringen Anzahl der Atome (jeweils ca. 80 Atome pro Isotop in den Analysedaten) wäre ein geringer Unterschied in der Besetzung hier allerdings nicht festzustellen.

\subsubsection{Platzbesetzung nach Isotopen getrennt}

Da für Nb-haltige Proben nur eine Platzbesetzung und zudem nur ein Isotop existiert, kann hier keinesfalls nach Isotopen unterschieden werden. Für Cr wäre eine Unterscheidung prinzipiell möglich, allerdings dominiert hier das ${ }^{52} \mathrm{Cr}$-Isotop mit 84\% Anteil sehr stark. Für Ag hingegen existieren 2 Isotope mit ungefähr gleichen Anteilen. ${ }^{107} \mathrm{Ag}$ sollte $51.8 \%$ und ${ }^{109} \mathrm{Ag}$ 48.2\% ausmachen. In Abb. 6.31 sind die AtomVicinity-Dichteprofile beider Isotope dargestellt. Ein statistisch signifikanter Unterschied ist nicht zu finden. Dies ist auch nicht überraschend, da man von der leicht veränderten Masse keinen Einfluss auf die Bindung erwartet.

\subsubsection{Platzbesetzung von $\mathrm{Cr}$}

Für $\mathrm{Ti}_{52} \mathrm{Al}_{45} \mathrm{Cr}_{3}$ erhält man unterschiedliche Platzbesetzungen. Da sich die Konzentrationen verschiedener Körner stark unterscheiden, macht es keinen Sinn, die Platzbesetzung an die nominelle Zusammensetzung zu koppeln. Die Zuordnung der 


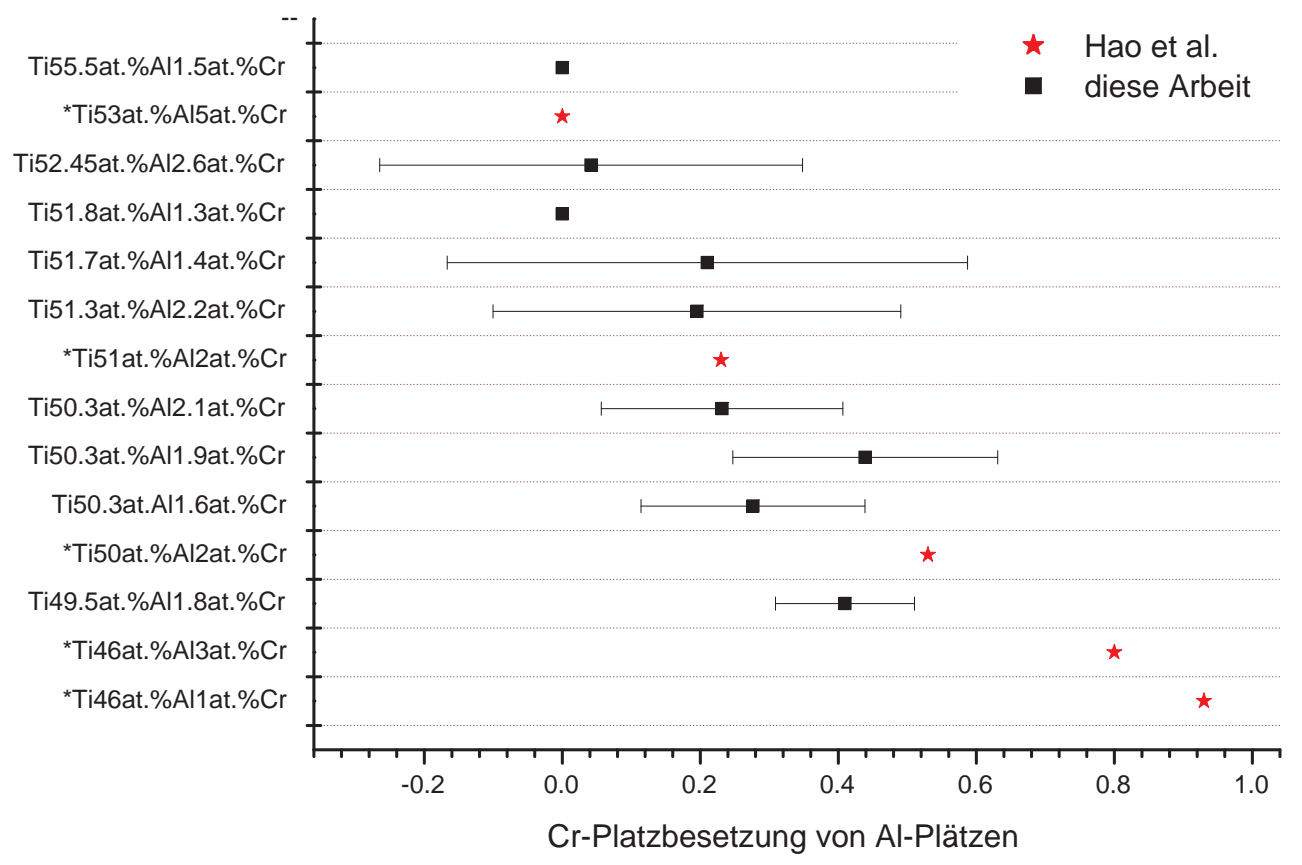

Abbildung 6.32: Platzbesetzung von Cr in verschiedenen TiAl $+X$-Legierungen aus Atomsondenmessungen. Die mit ${ }^{*}$ gekennzeicheten Werte von Hao et al. [Ha02000] sind zum Vergleich angegeben. Die Messwerte sind nach ihrem Ti/Al-Verhältnis sortiert.

Peaks zu einem Element ist schwieriger, da die Feldverdampfungsfeldstärken sich für verschiedene Platzbesetzungen unterscheiden. In Abschnitt 6.8.1.3 wurde festgestellt, dass Al-ärmere Körner eine Feldeverdampfungsfeldstärke von $\mathrm{Cr}$ zwischen $\mathrm{Ti}$ und $\mathrm{Al}$ besitzen, während ansonsten die Feldverdampfungsfeldstärken von Ti und Cr ähnlich groß erscheinen. Nimmt man an, dass naheliegenderweise Al-arme Körner einen größeren Cr-Anteil auf Al-Plätzen besitzen, so kann man davon ausgehen,dass Cr auf Ti-Plätzen ähnlich wie Titan verdampfen sollte, während Cr auf Al-Plätzen vor $\mathrm{Al}$ verdampfen sollte. Die Peakreihenfolge ist also $\mathrm{Cr}$ (von $\mathrm{Cr}$ auf Al-Plätzen)-Al$\mathrm{Ti} / \mathrm{Cr}$ (von Cr auf Ti-Plätzen). Simulationen in Kap. 7.6.6 werden dieses Verhalten qualitativ bestätigen. Die einzelnen Cr-Peaks lassen sich somit verschiedenen Plätzen zuordnen.

In Abb. 6.32 sind die Ergebnisse für unterschiedliche Konzentrationen zusammen mit ALCHEMI-Ergebnissen von Hao et al. [Hao2000] zum Vergleich angegeben. Die bevorzugte Platzbesetzung ändert sich offensichtlich in Abhängigkeit von der Zusammensetzung der Probe, dieses Verhalten ist auch aufgrund der ALCHEMIErgebnisse [Hao2000, Hao1999, Huan1991, Ross1996, Revi1993] und Berechnungen [Hao2000, Hao1999] anderer Gruppen zu erwarten. Für die Platzbesetzung ist nicht 
alleine das Ti/Al-Verhältnis ausschlaggebend. Erhöhte Cr-Konzentrationen erhöhen die Platzbesetzung von Al-Plätzen in den Al-reichen Proben ebenfalls ${ }^{8}$. Von Wesemann et at. [Wese1995] wurde mit Atomsonden-Feldionenmikroskopie (AP-FIM) bereits 1995 die Platzbesetzung von $\mathrm{Cr}$ in Ti46at.\%Al3at.\%Cr untersucht. Er findet eine starke Bevorzugung der Ti-Plätze. Dies ist durchaus zu verstehen, wenn man die Positionen der Peaks etwa in Abb. 6.33 betrachtet. Der mit Al zusammenhängende Peak verschmilzt oft mit dem zu Ti gehörenden Peak und ist zudem weit in Richtung Titan verschoben.

In Abb. 6.33 ist die sich verändernde Platzbesetzung für eine lange Messung angegeben, in der die Zusammensetzung sich ändert. Entsprechend ändert sich in diesem Korn auch die Platzbesetzung. Mit sinkender Al-Konzentration werden zunehmend Al-Plätze besetzt. An diesem Beispiel kann man gut erkennen, dass die Platzbesetzung immer nur ein Mittelwert ist. Sie kann auch innerhalb desselben Korns stark unterschiedlich sein.

\footnotetext{
${ }^{8} \mathrm{Im}$ Fall von Ti-reichen Proben werden verm. mit wachsender Cr-Konzentration mehr Ti-Plätze belegt. Dies kann aufgrund fehlender Messungen allerdings nicht experimentell bestätigt werden.
} 


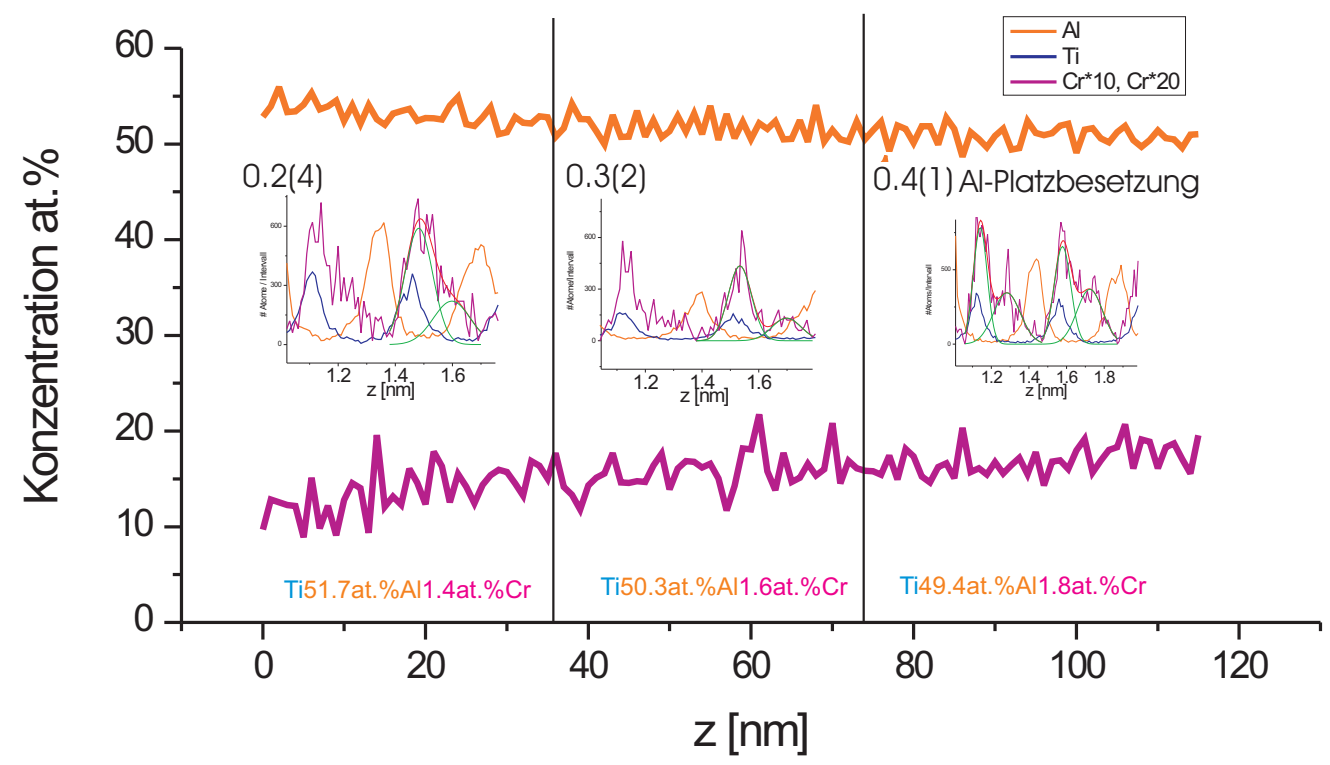

Abbildung 6.33: Platzbesetzung in einem TiAl+Cr-Korn mit einem Konzentrationsgradienten. Das analysierte Volumen hat die Maße $15 x 15 x 120 \mathrm{~nm}^{3}$. Die Konzentration von Cr ist mit 10, die Dichte in den, den jeweiligen Abschnitten zugeordneten, AtomVicinityDichteprofilen (Referenzatom: Al) mit 20 multipliziert. Die Al-Konzentration fällt von links nach rechts ab, während die Cr-Konzentration leicht ansteigt. Die durchschnittliche Konzentration in den durch die vertikalen Linien getrennten Bereichen ist angegeben. Die CrPlatzbesetzung von Al wächst mit fallender Al-Konzentration. 


\section{Kapitel 7}

\section{Simulationen}

Die in dieser Arbeit vorgestellten Feldverdamfungssimulationen und die daraus folgende Simulation von Atomsondendaten sind eine Weiterentwicklung der bereits 2005 entwickelten Modelle [Bol12005]. Im Grunde sind sie eine Modifikation der Müller-Schottky-Methode, die nun zusätzlich die lokale Umgebung der Atome durch Berücksichtigung der lokalen Bindungen einbezieht. Dies ist eine Erweiterung gegenüber dem Ansatz von Vurpillot et al. [Vurp1999, Vurp2000, Vurp2001a, Vurp2004b], die situationsunabhängige, feste Feldverdampfungsfeldstärken für jedes Element voraussetzen. Daraus ergibt sich allerdings die Notwendigkeit, die Bindungsenergien $\Lambda_{i}$ von verschiedenen Partnern zu berechnen bzw. die Simulation in Abhängigkeit dieser zu ändern, bis die Ergebnisse mit der Realität übereinstimmen. Will man ein eindeutiges Ergebnis erzielen, so ist diese Variation nur mit einem Parameter möglich.

Ein Ansatz ist, die Bindungsenergien einfach aus den Sublimationsenergien $\Lambda_{S u b}$ zu ermitteln. Dies wirft allerdings Probleme auf, da $\Lambda_{S u b}$ nur für reine Elemente bekannt ist. Für Legierungen kann bestenfalls ein gemeinsamer Wert angegeben werden. Deswegen ist eine rechnerische Ermittlung nötig. In dieser Arbeit wurde dafür mit der Dichtefunktionaltheorie (DFT) gearbeitet.

An dieser Stelle sei angemerkt, dass $\Lambda_{a-b}$ in dieser Arbeit genau die Energie bezeichnet, die für die Zerstörung der Bindung von einem Atom a zu einem Atom $b$ aufgebracht werden muss. An der Oberfläche ist diese Energie allerdings nicht mehr gültig. Da die Kohäsionsenergie in etwa gleich der Sublimationsenergie ist, [Liu1991, Toul2002] muss dort für eine Zerstörung der Bindung insgesamt eine Energie $2 \Lambda_{a-b}$ aufgewendet werden. Die verwendete Schreibweise hat den Vorteil, dass sie 
mit der am häufigsten verwendeten Notation von Paarpotentialen [Fark1994] kompatibel ist. Zudem erleichtert es die Überlegungen bei der Berechnung mit DFT, da die Energiebetrachtungen dort für Volumenatome stattfinden.

\subsection{Berechnung von Bindungsenergien}

Die Dichte-Funktional-Theorie (DFT) eignet sich besonders zur Berechnung von Gesamtenergien von kristallinen Materialien. Durch Vergleich der Ergebnisse unterschiedlicher Einheitszellen lassen sich Rückschlüsse auf die benötigten Bindungsenergien ziehen. Zur Berechnung verwendet wurde das kommerzielle Programm WIEN2k_09.1 [Blah2001] auf einem Computersystem mit einem Achtkern Xeon X5550-Prozessor bei $2.67 \mathrm{GHz}$ mit 23.4 GB RAM. Kleine 4-atomige Einheitszellen benötigen zur Berechnung auf diesem System etwa 1h. Die großen Superzellen mit 106 Atomen benötigen mehrere Tage. Dabei wurde pro Brillouin-Zone ein 18x18x18 Gitter verwendet. Die Berechnungen wurden von Zhiyong Zhu an der King Abdullah University in Saudi Arabien durchgeführt.

Die erhaltenen Energien wurden zur Konstruktion von Lennard-Jones-Potentialen benutzt.

\subsection{Modellierung der interatomaren Wechselwirkun- gen}

Auch wenn kompliziertere Modelle für Metalle bessere Potentiale liefern, wurden für diese Arbeit Lennard-Jones-Potentiale verwendet. Diese fallen mit der Entfernung relativ schnell ab. Im Prinzip wäre es ein besserer Ansatz, Embedded Atom Method (EAM)-Paarpotential [John1989, Pasi1992] zu verwenden, welche etwa von Farkas et. al [Fark1994, Fark1996] oder Zope et al. [Zope2003] für TiAl entwickelt wurden. In Abb. 7.1 sind die in dieser Arbeit verwendeten Lennard-Jones-Potentiale $J$ (Gl. 7.1)für TiAl (diese werden später in Kap. 7.6.2 hergeleitet) mit der NNBindungsenergie $\Lambda_{1 . N N}$ und dem NN-Abstand $d_{1 . N N}$ aufgetragen.

$$
J=\Lambda_{N N}\left\{\left(\frac{d_{1 . N N}}{d}\right)^{12}-2\left(\frac{d_{1 . N N}}{d}\right)^{6}\right\}
$$




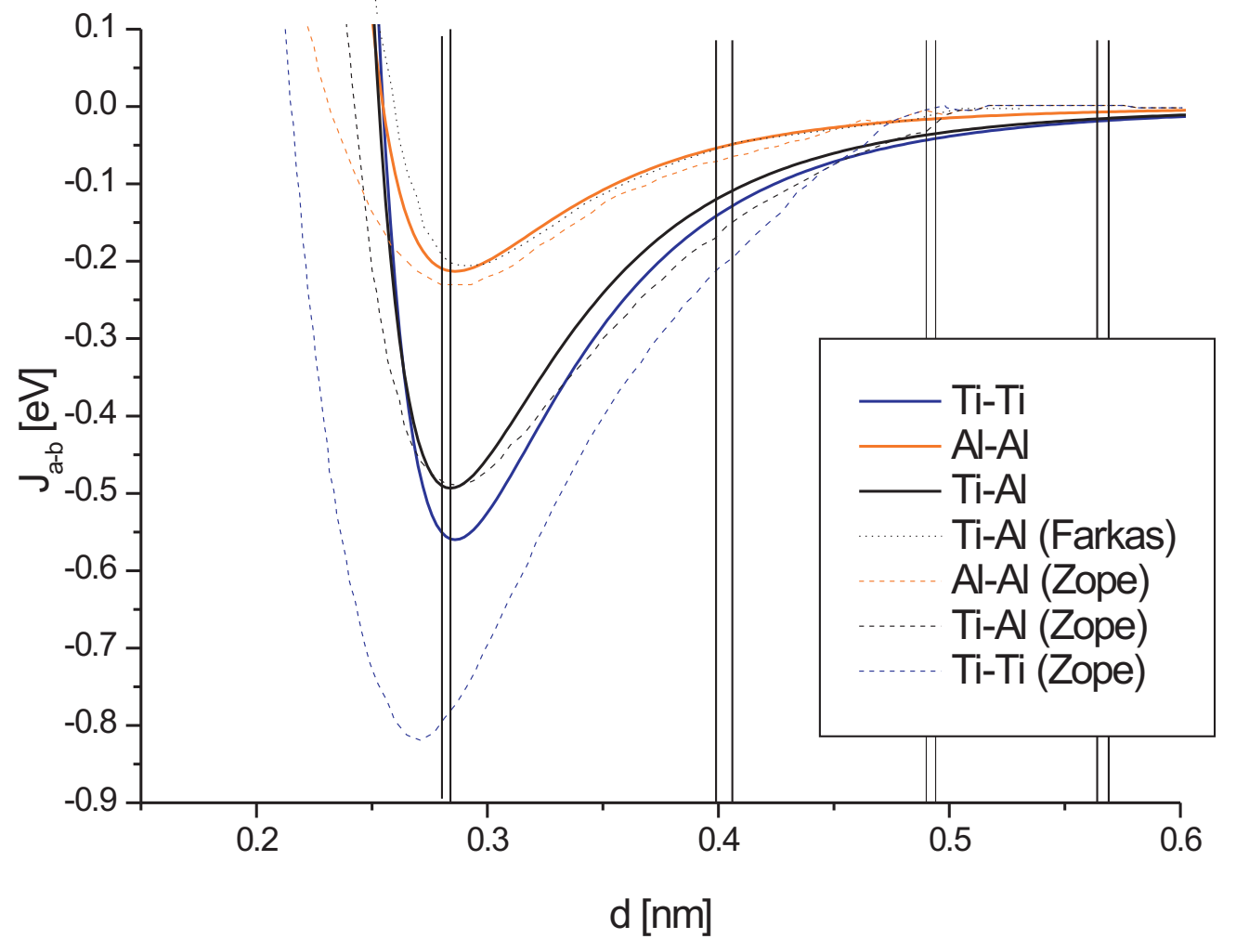

Abbildung 7.1: Die verwendeten Lennard-Jones-Potentiale aus Kap. \%.6.2 verglichen mit den EAM-Potentialen von Zope [Zope2003] und Farkas [Fark1994]. Die vertikalen Linien

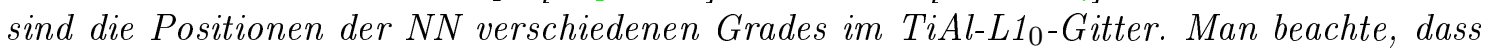
sich die NN wegen der Dehnung der Einheitszelle in [001]-Richtung, aufspalten. Dieser Effekt wird aber zunächst vernachlässigt, was angesichts der hier sichtbaren Unterschiede durchaus vertretbar scheint. Die Potentiale von Zope wurden auf das Minimum des Ti-Al-Potentials durch Division durch 1.325 normiert, was der Berücksichtigung höherer Nachbarn bis Grad 5 bei dem in dieser Arbeit benutzten Potential entspricht. 
Ebenfalls aufgetragen sind die von Farkas und die von Zope verwendeten Paarpotentiale. Die Unterschiede zwischen den Potentialen von Zope und Farkas sind offensichtlich groß. Im Unterschied zu den in dieser Arbeit verwendeten Potentialen sind die von Zope tiefer. Mit einem Lennard-Jones-Potential kann man das Potential von Farkas gut annähern. Die Potentiale von Zope fallen weniger schnell mit der Entfernung ab als mögliche $J(d)$. Die Potentiale aus beiden Quellen würden nach der Müller-Schottky-Formel (Gl.7.8) in Abschnitt 7.3.1 abweichende Werte für die Feldverdampfungsfeldstärken der reinen Materialien liefern. Während dabei Farkas die Feldverdampfungsfeldstärke eher unterschätzt, liegt sie bei Zope über dem zu erwartenden Wert. Zudem sind sie für Nachbarn, die weiter entfernt sind als die 2.NN nicht sinnvoll definiert, weswegen es wenig Sinn macht, sie in der Simulation, die bis zu den 5.NN reichen soll, zu verwenden. Die Ursache dafür ist, dass die Potentiale für eine endlich große Anzahl von Atomen berechnet wurden und somit besonders bei größeren Entfernungen wenig zuverlässig sind.

In Tabelle 7.1 ist das Lennard-Jones-Potential an den Positionen der $i$.NN verschiedener Grade $i$ für das kfz-Gitter angegeben. Alleine der Funktionswert von $J$ an diesen Stellen ist allerdings nicht ausschlaggebend für die Bedeutung der Nachbarn eines Grades. Wichtig ist auch die Anzahl. Dabei ist auffällig, dass die 1.NN, aufgrund ihrer geringen Anzahl (6 Stück) einen geringeren Beitrag zur Bindung eines Atoms leisten als die 2.NN (24 Stück).

Nachbarn von Grad 6 und höher spielen schließlich jedoch kaum noch eine Rolle für die Gesamtbindungsenergie eines Atoms.

\subsubsection{Grundlagen der DFT}

Die von Hohenberg und Kohn [Hohe1964, Kohn1965] entwickelte Dichtefunktionaltheorie beschreibt die Wechselwirkungen von Atomen über ihre Elektronen. Die Grundzustandsenergie des Gesamtsystems ist dabei ein Funktional der Elektronendichte $\rho_{0}$ im Grundzustand. Für jede andere Elektronendichte ist die Energie größer. Die Schrödingergleichungen für $N$ Quasielektronen (Kohn-Sham-Gleichungen ) lauten:

$$
\left(\frac{-\hbar^{2}}{2 m} \nabla^{2}+V_{e f f}(\vec{r})\right) \Psi_{i}(x, y, z)=\epsilon_{i} \Psi_{i}(\vec{r})
$$

Die Elektronendichte $\rho$ ist dabei

$$
\rho(\vec{r})=\sum_{i=1} N\left|\Psi_{i}(\vec{r})\right|^{2}
$$




\begin{tabular}{|l|l|l|l|l|}
\hline $\mathrm{NN}$ & $d$ [a.U.] & $\#$ NN & $J(d)$ [a.U.] & $\#$ NN. $J(d) / 12$ [a.U.] \\
\hline 1 & 0.71 & 12 & 1 & 1 \\
2 & 1 & 6 & 0.2344 & 0.1172 \\
3 & 1.23 & 24 & 0.0727 & 0.1454 \\
4 & 1.41 & 12 & 0.031 & 0.0310 \\
5 & 1.58 & 24 & 0.0159 & 0.0318 \\
6 & 1.73 & 8 & 0.0092 & 0.0061 \\
7 & 1.87 & 48 & 0.0058 & 0.0232 \\
8 & 2 & 6 & 0.0039 & 0.0020 \\
\hline
\end{tabular}

Tabelle 7.1: Lennard-Jones-Potential J (Gl. \%.1) an den Positionen d der NN unterschiedlichen Grades im kfz-Gitter. Anhand der Anzahl \# NN der NN und dem Funktionswert von $J$ kann die Bedeutung der $N N$ des jeweiligen Grades abgeschätzt werden. Die Werte von $J$ sind normiert auf die Gitterkonstante $d_{2 . N N}$ und die Energie $J\left(d_{1 . N N}\right)$ der 1 . NN.

Das effektive Potential $V_{\text {eff }}$ ist nun allerdings immer noch in unbekannter Weise von $\rho(\vec{r})$ abhängig. Deswegen bedarf es weiterer Näherungen. Eine gebräuchliche Näherung ist die Local Density Approximation (LDA), bei der das Funktional der Elektronendichte durch eine Funktion ersetzt wird. Für diese Arbeit wurde eine Generalized Gradient Approximation (GGA) nach Perdew et al. [Perd1996] verwendet. Dabei wird nicht nur die Funktion der Dichte sondern auch deren Gradient einbezogen. Die benötigten Parameter sind im WIEN2k-Programmpaket enthalten.

\subsubsection{Gitterparameter durch DFT}

Durch Vergleich der Gesamtenergien für verschiedenen Gitterparameter lässt sich die energetisch günstigste Gitterkonfiguration herausfinden. Für L1 $1_{0}$-TiAl erhält man eine in [001]-Richtung gedehnte Einheitszelle mit $a=0.3989 \mathrm{~nm}$ und $c=0.4057 \mathrm{~nm}$. Die Dehnung in $z$-Richtung ist somit 1.017, was mit experimentellen Werten [Meno1996] gut übereinstimmt.

Für $\mathrm{L}_{2}{ }_{2} \mathrm{Cu}_{3} \mathrm{Au}$ erhält man einen Gitterparameter von $0.3776 \mathrm{~nm}$, was dem experimentell bei Raumtemperatur bestimmten Wert von $0.3743 \mathrm{~nm}$ sehr nahe kommt. Diese Zelle ist nicht gestaucht.

Die bestimmten Gitterparameter wurden für die folgenden DFT-Berechnungen verwendet. 


\subsubsection{DFT-Bindungsenergien für TiAl durch Leerstellen}

Zur Bestimmung der Bindungsenergien für Ti-Al wurden verschiedene Ansätze ausprobiert. Entscheidend ist hierbei, dass sich die Gesamtenergie $G$ (Tabelle 7.2) sehr leicht bestimmen lässt. Durch Vergleich von $G$ für verschiedene Zellen lässt sich der Einfluss von Bindungsenergien $\Lambda_{a-b}$ auf $G$ herausfinden.

Zunächst werden Einheitszellen berechnet, in denen eine Leerstelle anstelle von Ti oder Al auftaucht. Deren $G$ kann mit dem $G$ einer Zelle, das nur das entfernte Atome enthält, verglichen werden. Dafür wird angenommen, dass bei der Entfernung eines Atoms die NN-Bindungen mit diesem Atom entfernt werden. Die Werte dieser Energien sind allerdings gegenüber der Gesamtenergie klein. Deswegen ist es nötig die Energie zu ermitteln, die das entfernte Atoms an sich besitzt. Entfernt man etwa aus der $\mathrm{Ti}_{2} \mathrm{Al}_{2}$-Zelle ein Ti-Atom, so werden $4 \mathrm{Ti}$-Ti-Bindungen und $8 \mathrm{Al}$-Ti-Bindungen beseitigt. Entfernt man stattdessen ein Al-Atoms, so werden 4 Al-Al und 8 Ti-AlBindungen beseitigt. Entfernt man weitere Atome, so muss berücksichtigt werden, dass Bindungen, die bereits nicht mehr vorhanden sind, nicht erneut beseitigt werden können. So kann man etwa durch $\left(G\left(T i_{2}\right)+G\left(T i_{2}\right)\right)-G\left(T i_{2} A l_{2}\right)=16 \Lambda_{T i-A l}=7.005$ $\mathrm{eV}$ die Differenz der Bindungsenergien berechnen. Den Wert einer Ti-Ti-Bindung kann man also durch $(G(T i A l)+G(T i))-G\left(T i_{2} A l_{2}\right)=4 \Lambda_{T i-T i}+8 \Lambda_{T i-A l}$ ausrechnen. Entsprechendes gilt für den Wert der Al-Al-Bindung.

Leider errechnet man auf diese Weise einen Energieunterschied, der zu groß ist. Im Grunde genommen vernachlässigt man mit dieser Methode die Leerstellenbildungsenergie. Diese sorgt für eine Absenkung der Energien, da sich die Elektronendichte der verbleibenden Atome dem durch das fehlende Atom neu entstandenen Leerraum anpasst. Es ist allerdings schwierig, zu sagen wie groß dieser Einfluss ist. Diese Methode kann also höchstens benutzt werden um eine untere Grenze für die Bindungsenergien anzugeben. Je nachdem, wie die Leerstellen in einer 4-atomigen $\mathrm{Ti}_{2} \mathrm{Al}_{2}$-Einheitszelle eingesetzt werden, ergibt sich durch Vergleich für die für die Bindungen $\Lambda_{T i-A l}=0.44 \mathrm{eV}, \Lambda_{T i-T i}=0.75 \mathrm{eV}$ und $\Lambda_{A l-A l}=0.40 \mathrm{eV}$.

Geht man davon aus, dass die Leerstellenbildungsenergie für Al und Ti vergleichbar ist, so lässt sich durch Vergleich zumindest der Unterschied zwischen den Ti-Ti- bzw. Al-Al-Bindungen berechnen, welcher bei $0.35 \mathrm{eV}$ liegt.

Hierbei ist, da die Randbedingungen periodisch sind, zu beachten, dass bei einer nur 4 Atome enthaltenden Zelle auch eine einfache Leerstelle der 2.NN von sich 


\begin{tabular}{|c|c|}
\hline Einheitszelle & Gesamtenergie $G$ [Ry] \\
\hline \multicolumn{2}{|l|}{ kleine Einheitszellen (4 Atome) } \\
\hline $\mathrm{Ti}_{4}$ & -6830.513764 \\
\hline $\mathrm{Al}_{4}$ & -1942.574861 \\
\hline $\mathrm{Ti}_{2} \mathrm{Al}_{2}$ & -4386.676364 \\
\hline $\mathrm{Ti}_{3} \mathrm{Al}$ & -5608.624860 \\
\hline $\mathrm{TiAl}_{3}$ & -3164.673767 \\
\hline $\mathrm{Ti}_{2}$ (2Al Leerstellen) & -3415.001936 \\
\hline $\mathrm{Al}_{2}(2 \mathrm{Ti}$ Leerstellen $)$ & -971.159555 \\
\hline TiAl (Al + Ti Leerstelle) & -2193.090198 \\
\hline $\mathrm{TiAl}_{2}$ (Ti Leerstelle) & -2678.885994 \\
\hline $\mathrm{Ti}($ ein $\mathrm{Ti}+2 \mathrm{Al}$ fehlen $)$ & -1707.311486 \\
\hline $\mathrm{Ti}_{2} \mathrm{Al}$ (Al Leerstelle) & -3900.815503 \\
\hline $\mathrm{Al}(2 \mathrm{Ti}+1 \mathrm{Al}$ fehlen $)$ & -485.485734 \\
\hline \multicolumn{2}{|l|}{ Superzellen (108 Atome) } \\
\hline $\mathrm{Ti}_{54} \mathrm{Al}_{54}$ & -118439.968538 \\
\hline $\mathrm{Ti}_{53} \mathrm{Al}_{54}$ (Ti Leerstelle) & -116732.193779 \\
\hline Ti (nur ein Ti Atom in der Zelle) & -1707.141627 \\
\hline $\mathrm{Ti}_{54} \mathrm{Al}_{53}$ (Al Leerstelle) & -117954.118132 \\
\hline $\mathrm{Al}$ (nur ein Al-Atom in der Zelle) & -485.365786 \\
\hline $\mathrm{Ti}_{55} \mathrm{Al}_{53}$ & -119661.883693 \\
\hline $\mathrm{Ti}_{53} \mathrm{Al}_{55}$ & -117217.963808 \\
\hline $\mathrm{Ti}_{53} \mathrm{AlAl}_{53} \mathrm{Ti}$ (jew. ein $\mathrm{Ti}$ und $\mathrm{Al}$ substituiert) & -118439.881893 \\
\hline $\mathrm{Ti}_{52} \mathrm{Al}_{54}$ & -115995.924620 \\
\hline $\mathrm{Ti}_{56} \mathrm{Al}_{52}$ & -120883.803742 \\
\hline
\end{tabular}

Tabelle 7.2: Mit DFT berechnete Gesamtenergien für verschiedene $L 1_{0}$ - Einheits- und Superzellen. $1[R y]=13.6[\mathrm{eV}]$. Die in den Superzellen entfernten Atome waren nächste oder übernächste Nachbarn. Man beachte, dass die angegebene Genauigkeit tatsächlich nötig ist, da die Energieunterschiede, die uns interessieren, erst in den Nachkommastellen beginnen. 
selbst (in allen Richtungen) ist. Bei 2 Leerstellen entsteht sogar ein flächiger Defekt. Deswegen ist es sinnvoll Vergleichsrechnungen mit Superzellen durchzuführen, um zu testen, inwieweit dieser Einfluss die Ergebnisse verfälschen kann. Die in Tabelle 7.2 berechneten Superzellen mit einer Leerstelle haben dabei den Vorteil, dass eine weitere Leerstelle erst in großer Entfernung auftaucht. Damit hat sie, falls die Wechselwirkung in Form eines Lennard-Jones-Potentials verläuft nach Tabelle 7.1 einen vernachlässigbaren Einfluss. Im Falle der 108-atomigen Superzellen liegt dieser weit unter 1\%. Für die Superzellen mit jeweils einer Leerstelle ergibt sich somit:

$$
\begin{gathered}
G\left(T i_{53} A l_{54}\right)+G(T i)-G\left(T i_{54} A l_{53}\right)-G(A l)=4 \Lambda_{N N, T i-T i}+6 \Lambda_{2 N N, T i-T i} \\
+8 \Lambda_{3 N N, T i-T i}-\left(4 \Lambda_{N N, A l-A l}+6 \Lambda_{2 N N, A l-A l}+8 \Lambda_{3 N N, A l-A l}\right)+\Lambda_{\text {sonst }}=2.0205 \mathrm{eV}
\end{gathered}
$$

Die Bindungsenergien höherer Nachbarn $\Lambda_{\text {sonst }}$ erschweren hier leider den Vergleich. Immerhin ist die Energie größer als für die 4-atomige-Zelle, was sich gut mit der nun erfolgten Einbindung der Bindungen weiter entfernter Nachbarn erklären lässt. Um die Bindungen weiter entfernter Nachbarn in Abhängigkeit des ersten Nachbarn angeben zu können, bietet es sich an, Lennard-Jones-Potentiale mit den Minima an der Position der NN anzusetzen. So kann man die Terme der höheren Nachbarn mit Tabelle 7.1 in Abhängigkeit vom ersten Nachbarn mit $\frac{6}{4} 0.234 \Lambda_{N N}+\frac{8}{4} 0.073 \Lambda_{N N}=$ $0.5 \Lambda_{N N}$ angeben und erhält

$1.5\left(4 \Lambda_{N N, T i-T i}-4 \Lambda_{N N, A l-A l}\right)=2.0205 \mathrm{eV} \Rightarrow \Lambda_{T i-T i}-\Lambda_{A l-A l}=0.34 \mathrm{eV}$.

Dies stimmt gut mit dem Ergebnis für die kleine Einheitszelle überein. Eine Verwendung von Lennard-Jones-Potentialen scheint somit durchaus gerechtfertigt.

\subsubsection{DFT-Bindungsenergien für TiAl durch Substitution}

Ein anderer Ansatz zur Berechnung der Bindungsenergien ist, anstelle von Leerstellen, fehlbesetzte Atome einzuführen, indem Ti-Plätze mit Al besetzt werden oder umgekehrt. Diese Methode hat den Vorteil, dass die Elektronen sich nicht innerhalb eines Leerraums neu organisieren und somit keine Leerstellenbildungsenergie hinzukommt. Allerdings ist natürlich das Ersetzen eines Atoms durch ein Anderes ebenfalls mit dem Problem verbunden, dass eine Elektronenkonfiguration durch eine Neue ersetzt wird. Dieser Fehler wird jedoch kleiner sein. Leider wird die Berechnung nun auch etwas aufwendiger, da nicht nur, wie beim Einfügen von Leerstellen, Bindungen verschwinden sondern auch neue Bindungen auftauchen. Deswegen folgt 
eine etwas ausführlichere Diskussion der Berechnungen. In Tabelle 7.2 sind die mit DFT berechnet Gesamtenergien $G$ aufgetragen. Hieraus lässt sich mit verschiedenen Betrachtungen die Bindungsenergie $\Lambda_{T i-A l}$ gemischter Partner in Abhängigkeit der Bindungsenergien der reinen Partner berechnen.

1) $\Lambda_{T i-A l}$ aus $T i_{4}, A l_{4}, T i_{2} A l_{2}$

Hierbei besitzen alle Zellen $4 !=24$ NN-Bindungen (jedes Atom mit den drei anderen. Bindungen, die bereits gezählt wurden, dürfen nicht erneut gezählt werden). Davon sind gewöhnlich $\frac{2}{3} \mathrm{zu}$ Atomen der anderen Art. In einer reinen Zelle fallen gegenüber der gemischt besetzten Zelle somit 16 gemischte NN-Besetzungen weg. Dafür kommen 16 gleichartige dazu.

$$
\begin{gathered}
G\left(T i_{4}\right)+G\left(A l_{4}\right)-2 G\left(T i_{2} A l_{2}\right)=16 \Lambda_{T i-T i}+16 \Lambda_{A l-A l}-32 \Lambda_{T i-A l}=3.593 \mathrm{eV} \\
\Rightarrow 2 \Lambda_{T i-A l}-\left(\Lambda_{T i-T i}+\Lambda_{A l-A l}\right)=0.225 \mathrm{eV}
\end{gathered}
$$

2) $\Lambda_{T i-A l}$ aus $T i_{3} A l, T i A l_{3}, T i_{2} A l_{2}$

In den zu $\frac{3}{4}$ korrekt besetzten Zellen fallen jeweils 4 gemischte Bindungen weg, die dafür als reine Bindungen im Vergleich zur 50/50 besetzten Zelle dazukommen. Der Unterschied zu 1) kann dadurch erklärt werden, dass die Zelle in [001]-Richtung gedehnt ist und somit die NN-Bindungsenergie im Grunde in 2 Varianten aufgespalten wird. In Fall 2) ist der Anteil der gemischten Bindungen in die nicht gedehnten [010] und [100]-Richtungen größer. Der NN-Abstand ist also im Durchschnitt dieser Bindungen kleiner. Da diese Bindungen für das Ergebnis wichtiger sind als die der gleichartigen Partner, ist das Ergebnis von 2) kleiner als 1):

$$
\begin{gathered}
G\left(T i_{3} A l\right)+G\left(T i A l_{3}\right)-2 G\left(T i_{2} A l_{2}\right)=4 \Lambda_{T i-T i}+4 \Lambda_{A l-A l}-8 \Lambda_{T i-A l}=0.736 \mathrm{eV} \\
\Rightarrow 2 \Lambda_{A l-T i}-\left(\Lambda_{T i-T i}+\Lambda_{A l-A l}\right)=0.184 \mathrm{eV}
\end{gathered}
$$

Setzt ein Lennard-Jones-Potential mit dem Minimum bei den gemischt nächsten Nachbarn an, so kann eine geringere Energie für Fall 2) reproduziert werden. Hierbei spielt vor allem der Unterschied bei den 2.NN eine Rolle, wie in Abb. 7.1 zu sehen (0.014 eV pro Ti-Al, 0.016 eV Ti-Ti-Bindung 0.01 pro Al-Al-Bindung). Da im Fall von $\mathrm{Cu}_{3} \mathrm{Au}$ (Kap.7.2.6), bei dem keine Aufspaltung der NN-Abstände vorliegt, jedoch ebenfalls eine Abweichung ermittelt wurde, ist es wahrscheinlich, dass der Unterschied durch die Veränderung der Elektronenverteilungen zustandekommen. 
3) $\Lambda_{T i-A l}$ aus den Superzellen $T i_{55} A l_{53}, T i_{53} A l_{55}, T i_{54} A l_{54}$

In diesem Fall besitzt die ungleichmäßig besetzte Zelle 8 zusätzliche reine Bindungen der Art des zugefügten Elements, dafür jedoch 4 reine Bindungen des entfernten Elements sowie 4 gemischte Bindungen weniger. $\Lambda_{\text {Sonst }}$ bezeichnet die Bindungen mit Nachbarn von höherer als der 1. Ordnung.

$$
\begin{gathered}
G\left(T i_{55} A l_{53}\right)+G\left(T i_{53} A l_{55}\right)-2 G\left(T i_{54} A l_{54}\right)=4 \Lambda_{T i-T i}+4 \Lambda_{A l-A l}-8 \Lambda_{T i-A l}+\Lambda_{\text {sonst }} \\
=1.219 \mathrm{eV} \Rightarrow 2 \Lambda_{A l-T i}-\left(\Lambda_{T i-T i}+\Lambda_{A l-A l}\right)+\Lambda_{\text {sonst }} / 4=0.305 \mathrm{eV}
\end{gathered}
$$

Geht man davon aus, dass die Potentiale eine Lennard-Jones-Form besitzen, kann man analog zu Kap. 7.2.3 den Anteil der $\Lambda_{\text {sonst }}$ herausrechnen. Es gilt $\Lambda_{N N, T i-A l}=$ $0.305 / 1.35 \mathrm{eV}=0.22 \mathrm{eV}$, was gut mit Fall 1) zusammenpasst.

4) $\Lambda_{T i-A l}$ aus den Superzellen $T i_{55} A l_{53} T i, T i_{54} A l_{54}$

Hier gilt das gleiche wie in 3) jedoch wird eine getauschte Bindung aufgehoben, da das Atom direkt daneben ebenfalls getauscht wird. Das Ergebnis weicht hier stärker ab, da durch die direkte Nachbarschaft von Ti und Al die Nahordnung wieder erhöht wird. Dadurch ist diese Situation energetisch erheblich günstiger und das Ergebnis somit eher als Maximalwert für eine mögliche Ti-Al-Bindung zu sehen.

$$
\begin{gathered}
G\left(T i_{53} A l A l_{53} T i\right)-G\left(T i_{54} A l_{54}\right)=3 \Lambda_{T i-T i}+3 \Lambda_{A l-A l}-6 \Lambda_{T i-A l}+\Lambda_{\text {sonst }}^{\prime}=1.179 \mathrm{eV} \\
\Rightarrow 2 \Lambda_{A l-T i}-\left(\Lambda_{T i-T i}+\Lambda_{A l-A l}\right)+\Lambda_{\text {sonst }}^{\prime} / 3=0.393 \mathrm{eV}
\end{gathered}
$$

5) $\Lambda_{T i-A l}$ aus den Superzellen $T i_{52} A l_{56} T i, T i_{56} A l_{52}, T i_{54} A l_{54}$

Diese Situation ähnelt der in 3) vorliegenden. Allerdings werden nur 7 gleichartige Bindungen der anderen Art vernichtet, da das zweite substituierte Atom den Platz eines NN der anderen Art einnimmt. Zusätzlich entsteht eine zusätzliche gleichartige Bindung. Insgesamt entstehen pro Superzelle somit 17 gleichartige Bindungen der Art des substituierten Atoms und 7 Bindungen der Art des anderen Atoms werden zerstört. Folglich werden gleichzeitig 10 gemischte Bindungen zerstört.

$$
\begin{gathered}
G\left(T i_{52} A l_{56}\right)+G\left(T i_{56} A l_{54}\right)-2 G\left(T i_{54} A l_{54}\right)=10 \Lambda_{T i-T i}+10 \Lambda_{A l-A l}-20 \Lambda_{T i-A l}+\lambda_{\text {sonst }}^{\prime \prime} \\
=2.840 \mathrm{eV} \Rightarrow 2 \Lambda_{A l-T i}-\left(\Lambda_{T i-T i}+\Lambda_{A l-A l}\right)+\lambda_{\text {sonst }}^{\prime \prime} / 10=0.284 \mathrm{eV}
\end{gathered}
$$

Da bei dieser Superzelle die Ordnung gegenüber 3) aufgrund der zusätzlichen gleichartigen NN geringer ist, ist diese Situation energetisch ungünstiger.

Generell sieht man, dass bei einer größeren berechneten Zelle zusätzlich noch die 


\begin{tabular}{|l|r|}
\hline Einheitszelle & Gesamtenergie $G[\mathrm{Ry}]$ \\
\hline kleine Ti-Al-Einheitzellen mit Fremdatomen (4 Atome) \\
\hline $\mathrm{Nb}_{4}$ & -30563.618643 \\
$\mathrm{Ti}_{2} \mathrm{Nb}_{2}$ & -18697.092383 \\
$\mathrm{Nb}_{2} \mathrm{Al}_{2}$ & -16253.258024 \\
$\mathrm{Cr}_{4}$ & -8406.816253 \\
$\mathrm{Ti}_{2} \mathrm{Cr}_{2}$ & -7618.706788 \\
$\mathrm{Cr}_{2} \mathrm{Al}_{2}$ & -5174.762177 \\
$\mathrm{Ag}_{4}$ & -42538.418893 \\
$\mathrm{Ti}_{2} \mathrm{Ag}_{2}$ & -24684.454699 \\
$\mathrm{Ag}_{2} \mathrm{Al}_{2}$ & -22240.503080 \\
\hline
\end{tabular}

Tabelle 7.3: Mit DFT berechnete Gesamtenergien für verschiedene L1 $1_{0}$ - Einheitszellen. 1 $[R y]=13.6$ [eV]. Die Gitterparameter sind in allen Berechnungen die für TiAl.

Bindungen höherer Ordnung hinzukommen. Die 2.NN waren im Fall der 4-atomigen Einheitszelle für jedes Atom das Atom selber.

Abschließend lässt sich sagen, dass ein Lennard-Jones-Potential mit dem Minimum bei den nächsten Nachbarn gemischter Art und einer Potentialtiefe, wie sie sich für den Fall einer 4 atomigen Einheitszelle in 1) errechnet, am sinnvollsten ist. Diese Potentialtiefe, welche $0.11 \mathrm{eV}$ tiefer als der Mittelwert der Potentiale der reinen Elemente an dieser Stelle ist, stimmt gut mit dem Ergebnis für die Superzelle in 3) überein. Die Ergebnisse der anderen Superzellen stützen die Aussage, dass bei den kleinen 4-atomigen Einheitszellen Bindungen höherer Nachbarn nicht enthalten sind.

\subsubsection{DFT-Bindungsenergien für TiAl $+\mathrm{X}$ durch Substitution}

Die Bindungsenergien der Zusätze $X=\mathrm{Cr}, \mathrm{Nb}, \mathrm{Ag}$ zu Ti bzw Al $\Lambda_{X-T i}, \Lambda_{X-A l}$ sind natürlich ebenfalls von Interesse. Deshalb wurden Bindungsenergien für Einheitszelllen berechnet, auf denen $\mathrm{Al}$ oder Ti durch jeweils $\mathrm{Ag}$, Nb oder $\mathrm{Cr}$ ersetzt wurden (Tabelle 7.3). Der Vorteil dieser Methode ist, dass dabei im Gegensatz zu einer Abschätzung aus den Sublimationsenergien, wie in [Boll2005, Kres2009], die tatsächlich vorliegende Struktur benutzt wird. Dies geschieht, ebenso wie für TiAl in Abschnitt 


\begin{tabular}{|l|l|}
\hline Bindungspartner A-B & Bindungsenergiedifferenz $2 \Lambda_{A-B}-\left(\Lambda_{A-A}+\Lambda_{B-B}\right)[\mathrm{eV}]$ \\
\hline Al-Ti & 0.22457 \\
Al-Nb & 0.27427 \\
Ti-Nb & 0.04452 \\
Al-Cr & 0.11329 \\
Ti-Cr & 0.07105 \\
Al-Ag & 0.01055 \\
Ti-Ag & -0.01978 (abstoßend) \\
\hline
\end{tabular}

Tabelle 7.4: Bindungsenergiedifferenzen in der TiAl-L1 $1_{0}$-Einheitszelle mit verschiedenen Partnern aus den Einheitszellen $\mathrm{Al}_{4}, \mathrm{Ti}_{4}, \mathrm{Ti}_{2} \mathrm{X}_{2}$ und $\mathrm{X}_{2} \mathrm{Ti} i_{2}$. Die Bindung Ti-Ag ist abstoßend im Vergleich zu reinen $L 1_{0}$-Ti und Ag-Phasen.

7.2.4 1), gemäß der Formeln

$$
\begin{aligned}
2 \Lambda_{T i-X}-\left(\Lambda_{T i-T i}+\Lambda_{X-X}\right) & =\frac{G\left(T i_{4}\right)+G\left(X_{4}\right)-2 G\left(T i_{2} X_{2}\right)}{16} \\
2 \Lambda_{A l-X}-\left(\Lambda_{A l-A l}+\Lambda_{X-X}\right) & =\frac{G\left(X_{4}\right)+G\left(A l_{4}\right)-2 G\left(X_{2} A l_{2}\right)}{16} .
\end{aligned}
$$

Man erhält die in Tabelle 7.4 aufgetragenen Resultate. Es lässt sich leicht erklären, dass Nb vor allem Ti-Plätze besetzt, da die Bindungen auf Ti-Plätzen mehr Al-TiBindungen enthalten und somit energetisch günstiger sind. Weder Ag noch Cr zeigen eine derart klare Tendenz, was erklärt weshalb sie sowohl Ti als auch Al-Plätze belegen können (Kap 6.10). Das Einbringen von $\mathrm{Ag}$ in die $\mathrm{L} 1_{0}$-Matrix ist zudem energetisch ungünstig. Dies erklärt, wieso sich $\mathrm{L}_{2}$-Ausscheidungen ausbilden (Kap. 6.1.2). Diese sind wegen des hohen Al-Anteils, und somit mehr Ag-Al-Bindungen, die günstigere Alternative.

Die $\Lambda_{a-b}$ können hier wieder als $\Lambda_{1 . N N, a-b}$ interpretiert werden, wie es der Vergleich der Superzelle von TiAl mit der Einheitszelle nahelegt.

\subsubsection{DFT-Bindungsenergien für $\mathrm{CuAu}$}

Ausgehend von der 4 Atome enthaltenden Einheitszelle wurden die Gesamtenergien $G$ für verschiedene Besetzungen und Fehlbesetzungen berechnet, die in Tabelle 7.5 aufgelistet sind. Aus den berechneten Energien lässt sich die $\mathrm{Cu}-\mathrm{Au}$ Bindungsenergie $\Lambda_{C u-A u}$ auf verschiedene Arten berechnen. Zum einen über die jeweils voll besetzten Zellen im Vergleich zur gemischt Besetzten durch

$G\left(C u_{4}\right)+G\left(A u_{4}\right)-2 G\left(C u_{2} A u_{2}\right)=16 \Lambda_{C u-C u}+16 \Lambda_{A u-A u}-32 \Lambda_{C u-A u}=3.498 \mathrm{eV}$ 


\begin{tabular}{|l|l|}
\hline Einheitszelle & Gesamtenergie $G[\mathrm{Ry}]$ \\
\hline $\mathrm{Cu}_{4}$ & -13240.197605 \\
$\mathrm{Cu}_{3} \mathrm{Au}$ & -48025.978994 \\
$\mathrm{Cu}_{2} \mathrm{Au}_{2}$ & -82811.690192 \\
$\mathrm{CuAu}$ & -117597.347522 \\
$\mathrm{Au}_{4}$ & -152382.925650 \\
\hline
\end{tabular}

Tabelle 7.5: Mit DFT berechnete Gesamtenergien für verschieden $L 1_{2}$ - Einheitszellen. 1 $[R y]=13.6[\mathrm{eV}]$.

$$
\Rightarrow 2 \Lambda_{C u-A u}-\left(\Lambda_{A u-A u}+\Lambda_{C u-C u}\right)=0.219 \mathrm{eV}
$$

zum Anderen durch die jeweils zu einem viertel besetzten im Vergleich mit den gemischt besetzten durch

$$
\begin{gathered}
G\left(C u_{3} A u\right)+G\left(C u A u_{3}\right)-2 G\left(C u_{2} A u_{2}\right)=4 \Lambda_{C u-C u}+4 \Lambda_{A u-A u}-8 \Lambda_{C u-A u}=0.704 \mathrm{eV} \\
\Rightarrow 2 \Lambda_{C u-A u}-\left(\Lambda_{A u-A u}+\Lambda_{C u-C u}\right)=0.176 \mathrm{eV} .
\end{gathered}
$$

Offensichtlich ist die Bedingung für das Vorliegen einer intermetallischen Phase nach Formel 2.1 erfüllt. Die Ergebnisse liegen erfreulich dicht beieinander, so dass man mit einem Wert von $\Lambda_{C u-A u}-\frac{\Lambda_{A u-A u}+\Lambda_{C u-C u}}{2}=0.10(1) \mathrm{eV}$ mit Sicherheit im richtigen Bereich liegt. Dadurch dass hier keine Stauchung der Einheitszelle vorliegt, kann dies, anders als im Fall von TiAl (Kap. 7.2.4), auch nicht als möglicher Grund angeführt werden. Die Abweichung ist damit durch die Unterschiede der Elektronenverteilungen begründet.

\subsection{Vereinfachtes Modell einer Atomsonde}

Zunächst sind einige Vereinfachungen nötig, um ein berechenbares Modell der Atomsonde zu erhalten. Der Vergrößerungsfaktor $G_{0}$ aus Gleichung 3.2 wird auf 1 gesetzt. Dies entspricht einem Abstand Detektor-Spitze $L$, der gleich dem Apexradius $R$ ist.

$$
L=R \Rightarrow \eta=\frac{L}{R}=1 \text {. Es gilt somit } x=X \text { und } y=Y .
$$

Als einfaches Modell für die Spitze wird eine Kugelkappe mit Radius $R_{0}$ auf einem Zylinder mit Radius $R_{0}$ gewählt, deren Geometrie sich während des Verdampfungsprozesses nicht ändert. Somit ändert sich der Krümmungsradius $R=R_{0}$ während des Verdampfens nicht. Nach Gleichung 3.1 bleibt auch die Spannung $V=R E_{\text {Evap }} \beta$ 


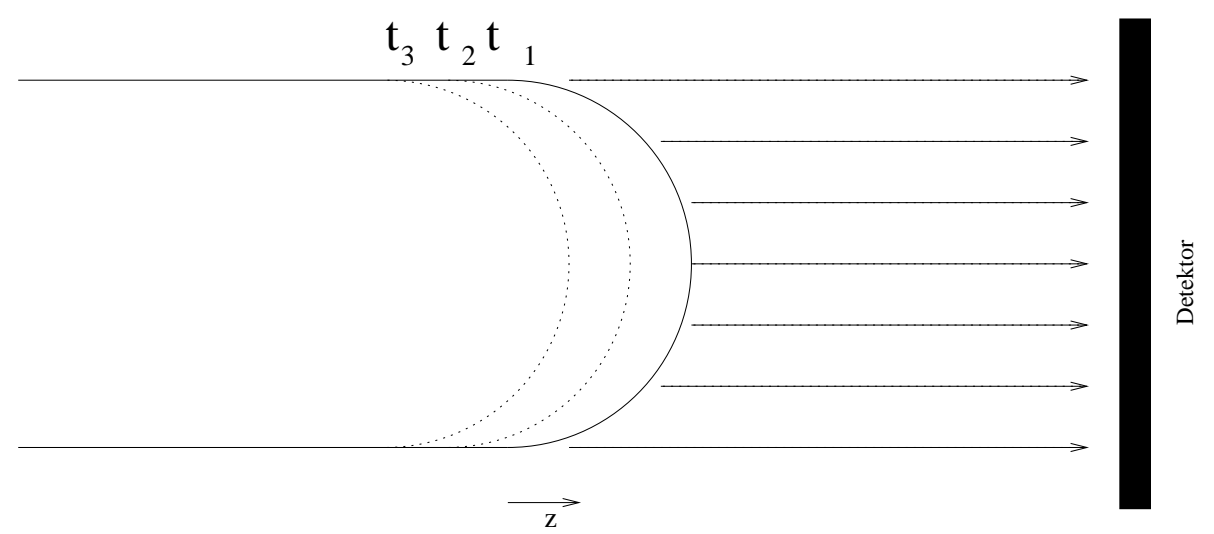

Abbildung 7.2: Das vereinfachte TAP: Die Pfeile symbolisieren nicht die tatsächlichen Feldlinien, die gekrümmt wären sondern sollen die simulierte Abbildung der Atome auf den Detektor darstellen. $t_{1}<t_{2}<t_{3}$ zeigen die Spitzenoberfläche zu verschiedenen Zeitpunkten.

während der Feldverdampfung konstant. Die zeitliche Entwicklung der Morphologie und das Abbildungsverhalten einer solchen Spitze ist in Abb. 7.2 dargestellt.

Aufgrund des Vergrößerungsfaktors von 1 ist die Detektorfläche $S_{D}$ durch $\pi R^{2}$ gegeben. Die Detektionseffizienz $Q$ wird auf 1 gesetzt. Weil die Spitze nicht verkippt wird, ist zudem $m=0$. Somit kann man $K_{0}$ aus Gl. 3.3 vereinfachen zu

$$
K_{0}=\frac{\left(L E_{E v a p} \beta\right)^{2}}{S_{D} Q(m+1)^{2}} V_{a t}=\frac{V_{i}^{2}}{\pi R^{2}} V_{a t}
$$

Damit gilt für die Rekonstruktion der z-Komponente des N-ten Atoms mit Gl. 3.6

$$
z=\frac{V_{a t}}{\pi R^{2}}(N-1)+\frac{x^{2}+y^{2}}{2 R} .
$$

Diese Gleichung ist angenehmerweise von dem Parameter $E_{\text {Evap }} \beta$ unabhängig. Das Atomvolumen $V_{a t}$ bleibt konstant und spielt somit keine Rolle. Der Parameter $\frac{V_{a t}}{\pi R^{2}}$ ist also die Distanz, um die die ideale Kugelkappe durch die Verdampfung eines Atoms in $z$-Richtung verschoben wird.

\subsubsection{Der Müller-Schottky Mechanismus}

Die Feldverdampfung eines Atoms wird durch die am Ort des Atoms herrschende elektrischen Feldstärke $E$ bestimmt. Ist $E$ größer als die Feldverdampfungsfeldstärke $E_{E v a p}$, so wird das Atom von der Oberfläche entfernt. Diese Feldstärke hängt nach Müller et al. [Muel1969] vom Ionisationsgrad $n$ des Atoms ab.

$$
E_{\text {evap }, n}=\frac{4 \pi \epsilon_{0}}{n^{3} e^{3}}\left(U_{n}^{\prime}\right)^{2} .
$$


Dabei ist $\epsilon_{0}$ die Feldkonstante und $e$ die Elementarladung. Die Energie $U_{n}$ setzt sich aus der Sublimationsenergie $\Lambda_{S u b}$, den Ionisationsenergien $I_{i}$ für die jeweiligen Ionisationsgrade und der Austrittsarbeit der Elektronen $\Phi$ zusammen.

$$
U_{n}=\Lambda_{S u b}+\sum_{i=1}^{n} I_{i}-n \Phi .
$$

In neueren Varianten des „Image Hump“-Modells, wie es von Zurlev et al. [Zurl2003] genutzt wird, wird $\Lambda$ als im elektrischen Feld abgesenkt betrachtet. Diese Absenkung ist proportional zu $E^{2}$. Allerdings ist der genaue Zusammenhang in unserem Fall mit drei verschiedenen Elementen nicht bekannt. Zudem ergibt sich für den Fall, dass die Proportionalitätskonstante für alle Materialien identisch gewählt wird, kein Einfluss auf die Verdampungsreihenfolge. Deswegen lassen wir den Einfluss des Feldes auf die Bindungsenergie unberücksichtigt.

Wendet man Gl. 7.7 mit Gl. 7.8 an, so erhält man bei $\mathrm{W}(n=3)$, Nb $(n=2)$, $\mathrm{Ti}(n=2)$ und $\mathrm{Cu}(n=1)$ eine gute Übereinstimmung mit den experimentellen Werten. Bei Al jedoch erhält man einen nicht zu erklärenden Wert für die Feldverdamfungsfeldstärke von $19 \mathrm{~V} / \mathrm{nm}$ (für $n=1$ ) bei gemessenen $33 \mathrm{~V} / \mathrm{nm}$ [Mill1996]. Auch für Au weicht $E_{\text {Evap }}$ mit gemessenen $35 \mathrm{~V} / \mathrm{nm}$ bei erwarteten $53 \mathrm{~V} / \mathrm{nm}$ (mit $n=1)$ stark ab. Beide Elemente wurden in den in dieser Arbeit experimentell gemessenen Legierungen zudem ausschließlich mit $n=2$ detektiert (Kap 6.4.3). Es lässt sich somit zurecht Kritik an diesem Modell üben, wie sie von Biswas et. al [Bisw1982] sowie Forbes [Forb1982] vorgebracht wird.

Da wir anstreben, Mischkristalle zu simulieren, ist die Austrittsarbeit $\Phi$ formal für jedes Element unterschiedlich. Es stellt sich hier natürlich die Frage, ob dies eine gute Annahme ist. Eigentlich kann man, zumindest makroskopisch, eine einheitliche Austrittsarbeit für die Elektronen erwarten. Da die Verdampfung jedoch lokal für ein Atom stattfindet, nehmen wir an, dass die zu den jeweiligen Materialien gehörenden Austrittsarbeiten gelten. Für das $\mathrm{CuAu}$-System ist der Unterschied zwischen den Austrittsarbeiten [Mill1996] mit $0.3 \mathrm{eV}$ zudem klein. Bei Ti, Al und Nb beträgt der Unterschied sogar nur vernachlässigbare $0.1 \mathrm{eV}$ Lediglich in $\mathrm{TiAl}$ mit $\mathrm{Ag}$ oder $\mathrm{Cr}$ treten Unterschiede bis zu $0.5 \mathrm{eV}$ auf.

\subsubsection{Das verwendete Feldverdampfungsmodell}

Die TAP-Rekonstruktion erfolgt zuallererst über die Reihenfolge, in der die Atome detektiert werden. Somit kann unser Modell, das der Simulation von TAP-Daten 
dient, darauf beschränkt werden, die zeitliche Reihenfolge der Feldverdampfung zu bestimmen. Die Feldverdampfung wird schrittweise durchgeführt, wobei in jedem Schritt ein Atom verdampft. Dies entspricht einer Feldverteilung, bei der nur für ein Atom die Feldstärke $E$ größer als $E_{\text {Evap }}$ ist.

Die zur Berechnung von $E_{\text {Evap }}$ nötige Bindungsenergie ist nicht für jedes Atom in einer Legierung identisch. Sie ist sogar, wie in Kap. 6.4.4.1 für W gezeigt, noch nicht einmal für dasselbe Element überall gleich. Dies liegt daran, dass die Umgebungen der Atome beim Verdampfen nicht identisch sind. Aus diesem Grund werden, anstelle einer für jedes Element separat festgelegten Bindungsenergie, die Bindungsenergien $\Lambda_{i}$ mit den Nachbarn herangezogen.

Da die Berechnung von $E(x, y, z)$ sehr aufwendig ist, wird angenommen, dass der Apexbereich immer nahezu halbkugelförmig ist. Zudem soll auf der idealen $\mathrm{Ku}$ gelkappe -anders als von Dyke et. al [Dyke1953] für eine Sphäre auf einem Konus berechnet- die Feldstärke überall gleich sein. Setzt man, was in experimentellen Messungen oft der Fall ist, voraus, dass der Ionisationsgrad $n$ für alle Atome gleich ist, so lässt sich das Modell weiter vereinfachen. Da $E_{E v a p, n} \propto\left(U_{n}^{*}\right)^{2}$ und keine Terme anderer Ordnung existieren, ist es für die Bestimmung der Evaporationsreihenfolge glücklicherweise nach Gl. 7.7 möglich anstelle $E_{\text {Evap,n }}$ die Energie $U_{n}^{\star}$ heranzuziehen.

Da so nach einigen Feldverdampfungsvorgängen allerdings keine ideale halbkugelförmige Spitze mehr vorliegt, muss der Einfluss eines äußeren Feldes energetisch einbezogen werden. Dazu wird angenommen, dass das Feld an den Stellen der Atome, die oberhalb der idealen Oberfläche liegen, größer ist und an den Stellen der Atome, die unterhalb der idealen Oberfläche liegen, geringer ist. Dies kann als energetisch günstigere bzw. ungünstigere Position interpretiert werden. Somit wird ein linearer Strafterm $D \Delta z$ eingeführt, der diese Energie bestimmt. Dabei ist $\Delta z$ der Abstand des jeweiligen Atoms zur Kugelkappe in $z$-Richtung und $D>0$ ein noch zu bestimmender Straffaktor. Ein $\Delta z>0$ ergibt sich für über die Kugelkappe hinausragende Atome, ein $\Delta z<0$ für niedrig gelegene Atome.

Eine weitere wichtige Einschränkung ist, dass Atome nur dann verdampfen dürfen, wenn sie auf der Oberfläche liegen. Für ein kfz-basiertes Gitter bedeutet dies, dass weniger als $75 \%$ der NN vorhanden sind. Es macht keinen Sinn, diese Grenze höher zu setzen, da dann in gemischten Ebenen Atome verdampfen könnten, die in der gleichen Ebene noch von allen Seiten von anderen Atomen umgeben sind. Dies geschieht, wie man gut an den FIM-Bilder der gemischten $\mathrm{Cu} / \mathrm{Au}$-Ebene in [110]- 
Richtungen (Abb. 6.10) erkennen kann, offensichtlich nicht. Liegt ein einkomponentiges Material vor, so ist diese Bedingung nicht nötig, da die Unterschiede in den Feldverdampfungsfeldstärken zu gering sind und zudem nur für verschiedene Pole existieren. Zusätzlich muss noch als Randbedingung ein stabiler Schaft angenommen werden. Randatome des Schaftes könnten ansonsten zu früh feldverdampfen. Diese Atome dürfen erst evaporieren, wenn die ideale Kugelkappe so weit nach unten gewandert ist, dass sie oberhalb dieser Kugelkappe liegen.

Zur Berechnung der Reihenfolge wird nun folgende auf Gl. 7.8 aufbauende Energie für ein Atom $j$ benutzt.

$$
U_{n}=\sum_{i} \Lambda_{i}+\sum_{j=1}^{n} I_{j}-n \Phi+U_{k i n}-D \Delta z
$$

Hierbei ist $U_{\text {kin }}$ ein zufälliger Wert, der, im Vergleich mit den anderen Termen, vernachlässigbar klein ist und $\Lambda_{i}$ die Bindungsenergie der nächsten Nachbarn bis zu einem vorher bestimmten Abstand.

Der Term $U_{k i n}$ dient hierbei lediglich dazu, eine zufällige Reihenfolge zu erzeugen, wenn mehrere Atome einen ansonsten identischen Energiewert $U_{n}$ besitzen. Man kann diesen Wert auch als temperaturabhängige kinetische Energie ansehen. Die Größenordnung solcher Energien liegt mit $\frac{3}{2} k T=0.005 \mathrm{eV}$ bei $40 \mathrm{~K}$ um einige Größenordnungen unter den anderen Energien, die im Bereich von $0.1 \mathrm{eV}$ oder darüber liegen.

Da das Simulieren einer kompletten Spitze sehr aufwendig ist, ist es sinnvoll, nur einen zylinderförmigen Ausschnitt (wie in Abb. 7.3) zu simulieren. Außerhalb dieses Bereiches wird eine halbkugelförmige Kappe angenommen. $\mathrm{Zu}$ klein sollte dieser Ausschnitt nicht sein, da im Extremfall nur eine Gitterebene an der Oberfläche wäre. Typischerweise wurde ein Radius des Zylinders von etwa $R_{Z y l}=\frac{7}{8} R$ gewählt. Dies entspricht einer Beschneidung des Winkelbereichs der Spitze von $180^{\circ}$ auf $122^{\circ}$, was mit dem FIM-Sichtbereich vergleichbar ist.

\subsubsection{Ablauf der Simulation}

Zunächst wird die gewünschte Kristallstruktur simuliert, in die gewünschte Orientierung verdreht und anschließend ein Block herausgeschnitten, aus dem die zylindrische Spitze mit Kappe herausgeschnitten wird. Dieser Schritt erfolgt in einem 


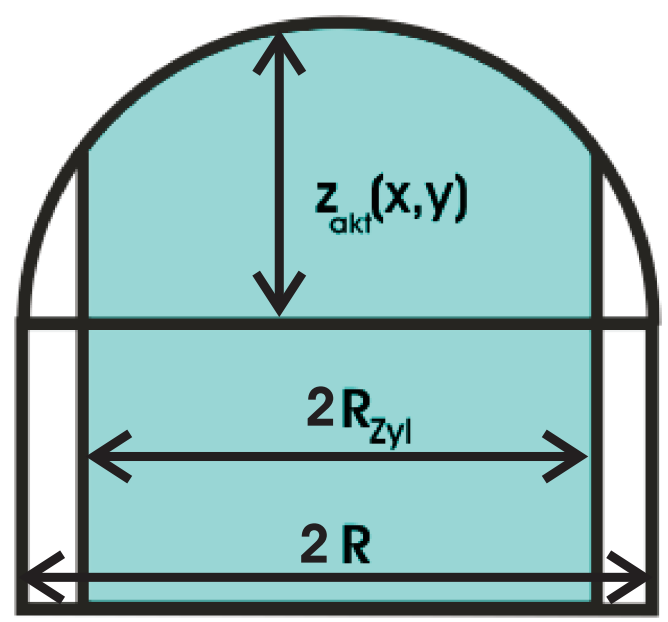

Abbildung 7.3: Spitzenprofil in der Seitenansicht. Das simulierte Volumen kann auf den markierten Bereich beschränkt werden.

von der Simulation getrennten Modul. Dies ermöglicht eine größere Flexibilität bei der Auswahl der Ausgangsstrukturen.

Für diesen Datensatz wird die Startsituation für alle Atome berechnet. Dafür werden für jedes Atom alle NN $i$ bis zum gewünschten Grad bestimmt und in einer zum Atom gehörigen Liste gespeichert. Danach wird für jedes Atom, in Abhängigkeit seiner Nachbarn, die Startenergie gesetzt, die hier noch nicht den Strafenergieterm enthält:

$$
U_{n, j}=\sum_{i} \Lambda_{i-j} \sum_{j=1}^{n} I_{j}-n \Phi+U_{k i n, j}
$$

Nach Abschluss dieser Vorarbeiten kann die eigentliche Simulation beginnen. Der Ablauf der Simulation ist in Abb. 7.4 in einem Nassi-Schneidermann-Diagramm dargestellt.

Dabei wird der im folgenden beschriebene Schritt solange wiederholt, bis die gewünschte Zahl von Atomen evaporiert ist.

Zur Berechnung der Strafenergie der Atome muss dabei zunächst die aktuelle Position der Kugelkappe $z_{a k t}(x, y)$ berechnet werden, die nach jedem Evaporationsschritt um $\frac{V_{a t}}{\pi R^{2}}$ verringert wird. Dabei ist $\Delta z_{j}=z_{j}-z_{a k t}(x, y)$, wobei $z_{a k t}(x, y)=$ $\left.\sqrt{R^{2}-x_{j}^{2}+y_{j}^{2}}\right)$ von der Position des jeweiligen Atoms $(x, y, z)$ abhängig. Es wird also das an der Oberfläche liegende Atom $k$ mit dem niedrigsten Wert von

$$
U_{n, j}^{\prime}=U_{n, j}-D \Delta z_{j}
$$


SimTAP — Programmteil zur Verdampfung

\begin{tabular}{|l|l|}
\hline \multicolumn{2}{|l|}{ Erstelle Spitze mit Kugelkappe, initialisiere Atomdaten mit NN und Energien } \\
\hline \multicolumn{2}{|l|}{$\begin{array}{l}\text { Wähle das erste Atom } j=1 \\
\text { Bst akt. Energie von Atom } j \text { bisher die niedrigste, besitzt ausreichend wenig NN und ist } \\
\text { Bein Schaftatom, das nicht evaporiert werden darf? }\end{array}$} \\
$\begin{array}{l}\text { Wähle Atom } k \leftarrow j \text { als zu evaporierendes Atom } \\
\text { Wis alle Atome bearbeitet sind }\end{array}$ \\
$\begin{array}{l}\text { Evaporiere zu evaporierendes Atom } k \\
\text { ändere Energie aller NN von } k \\
\text { setze Zeiger der NN auf Atom } k \text { auf NULL }\end{array}$ \\
bis gewählte Atomanzahl evaporiert
\end{tabular}

Abbildung 7.4: Struktogramm zum Feldverdampfungsteil von SimTAP

bestimmt, das zudem kein Schaftrandatom und kein Volumenatom ist. Dieses wird evaporiert. Anschließend werden die Energien aller NN $i$ diese Atoms um den jeweiligen Wert $\Lambda_{k-i}$ verringert:

$$
U_{n, i} \leftarrow U_{n, i}-\Lambda_{k-i}
$$

Der Programmcode, insbesondere für die Datenstrukturen, wird in Ausschnitten im Anhang A präsentiert.

\subsection{Bestimmung von $D$ mit Wolfram}

Um die Simulation zu testen, und um den Straffaktor $D$ zu kalibrieren, wurde die Feldverdampfung von Wolfram simuliert. Dabei sollten sich aus der unterschiedlichen Sublimationsenergie für verschieden Richtungen Unterschiede bewirken. Diese wurden z.B. von Terblans et. al. [Terb2003] für verschiedene Richtungen in Al berechnet.

Die Ionisationsenergien für Wolfram sind $I_{1}=7.98 \mathrm{eV}, I_{2}=18 \mathrm{eV}$ und $I_{3}=24 \mathrm{eV}$. Die Austrittsarbeit für Elektronen ist $\Phi=4.5 \mathrm{eV}$. Da Wolfram fast nur 3-fach ionisiert 


\begin{tabular}{|l|l|l|l|l|}
\hline $\mathrm{NN}$ & $d$ [a.U.] & $\#$ NN & $J(d)$ [a.U.] & $\#$ NN. $J(d) / 8$ [a.U.] \\
\hline 1 & 0.866 & 8 & 1 & 1 \\
2 & 1 & 6 & 0.6652 & 0.4989 \\
3 & 1.41 & 12 & 0.1027 & 0.15405 \\
4 & 1.658 & 24 & 0.0402 & 0.1206 \\
5 & 1.732 & 8 & 0.031 & 0.031 \\
6 & 2 & 6 & 0.0131 & 0.009825 \\
7 & 2.179 & 24 & 0.0067 & 0.0201 \\
\hline
\end{tabular}

Tabelle 7.6: Lennard-Jones-Potential J (Gl\%.1) an den Positionen d der NN unterschiedlichen Grades im krz-Gitter. Anhand der Anzahl \# NN der NN und dem Wert von J kann die Bedeutung der NN des jeweiligen Grades abgeschätzt werden. Die Werte von J sind normiert auf die Gitterkonstante $d_{2 . N N}$ und die Energie $J\left(d_{1 . N N}\right)$ der 1. NN.

gemessen wird, ist also $\sum_{i=1}^{3} I_{i}-3 \Phi=36.5 \mathrm{eV}$. Die Sublimationsenergie ist $8.66 \mathrm{eV}$. Die Anzahl der NN im krz-Gitter ist 8. In Tabelle 7.6 ist aufgelistet, wie sich das LennardJones-Potential in krz-Gittern verhält. Sinnvoll ist es hier bis zum 4.NN zu rechnen, da weiter entfernte Nachbarn $<5 \%$ Veränderung bedeuten. Insgesamt besitzt bei dieser Berücksichtigung jedes Atom das 1.774-fache der NN-Bindungsenergie. Die Bindungsenergie der NN (und somit das Minimum des Lennard-Jones-Potentials) ist somit $\Lambda_{1 . N N, W-W}=8.66 /\left(8^{*} 1.774\right) \mathrm{eV}=0.61 \mathrm{eV}$. Zur Überprüfung der Plausiblität dieses Potentials muss eine typische Evaporationssitaution mit etwas weniger als der Hälfte, also vier, der NN herangezogen werden. Ein solches Atom ist typischerweise ein Eckenatom für das die Bindungsenergie, die in diesem Fall die Sublimationsenergie ist, nach Liu et al. [Liu1991] nicht wesentlich von der Kohäsionsenergie abweichen darf, und es in unserer Simulation mit $\Lambda_{S u b}=2 * 4 * 1.774 * 0.61 \mathrm{eV}=8.65 \mathrm{eV}$ auch nicht tut. Der Faktor 2 stammt, wie schon zu Anfang des Kapitels gesagt, aus der Notation und ist nötig, da die Sublimationsenergie gleich der Gesamtbindungssenergie ist. Es wurde ein Spitzenradius von $4 \mathrm{~nm}$ mit einem Zylinderradius von $3.5 \mathrm{~nm}$ simuliert. Die Spitzenlänge war $16 \mathrm{~nm}$.

In einer Simulationsreihe in Abb. 7.5 mit verschiedenen Straffaktoren für die [112]Richtung erkennt man, dass bei kleinen $D$ die Verdampfung scheibenweise mit (110)Scheiben erfolgt. In diesem Fall kann man in den AtomVicinity-Dichteprofilen der rekonstruierten Atomdaten bei anderen Polen keinerlei Ebenen und in der Abb. keine Terrassen unterscheiden. Wählt man größere $D$, so erkennt man Ebenen an anderen Polen. Allerdings kann der Unterschied der AtomVicinity-Ebenenabstände mit größeren Ebenenabständen für den (112)-Pol wie in Kap. 6.4.4.1 in Tabelle 6.1 durch 

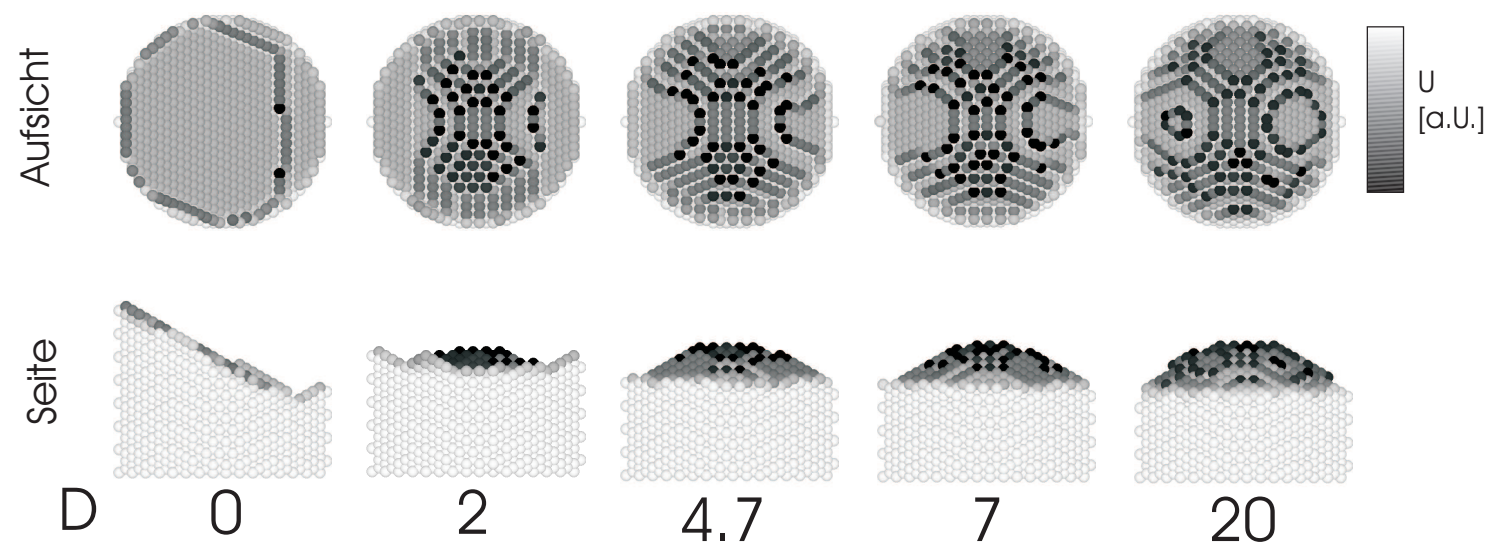

$[\mathrm{eV} / \mathrm{nm}]$

Abbildung 7.5: Simulierte W-Spitze in [112]-Richtung nach 10000 Evaporationsereignissen für verschiedene Straffaktoren D. Man erkennt, dass bei $D=0$ die Evaporation über die (110)-Ebene stattfindet. Bei $D=0.2$ bildet sich bereits eine Erhebung an der Position des (112)-Pols (in der Mitte) heraus, die in einer Mulde liegt. Eine weitere Erhöhung von $D$ bewirkt eine immer bessere Annäherung an die ideale Halbkugelform der Spitze. In der Darstellung ist die Gesamtenergie $U$ mit weiß für hohe und schwarz für niedrige Werte codiert. Schwarze Atome werden innerhalb eines der nächsten Evaporationsschritte abgelöst werden. Die Schaftatome sind weiß, da sie noch nicht berücksichtigt werden.

Variation von $D$ nicht reproduziert werden. Der Ebenenabstand nähert sich stattdessen von kleineren Werten asymptotisch dem erwarteten Verhältnis $d_{112}=d_{110} / \sqrt{3}$ an. Der Grund ist, dass die Unterschiede auf dem lokalen Vergrößerungseffekt beruhen, der in der Simulation nicht reproduziert werden kann, da $(x, y)$-Werte nicht verändert werden. Dass die Ebenenabstände dennoch abweichen liegt daran, dass bei kleinen Straffaktoren die (110)-Ebenen an allen Stellen dominieren. Zumindest lässt sich so eine untere Grenze für $D$ angeben, da im Experiment die Ebenen definitiv unterscheidbar sind. $D$ muss somit größer als $1 \mathrm{eV} / \mathrm{nm}$ sein.

Um einen besseren Zusammenhang zwischen Simulation und Experiment herzustellen, bietet sich eine Betrachtung der Veränderung der lokalen Krümmung an ${ }^{1}$. Dafür wurden zwei Simulationen durchgeführt. Bei der Einen befand sich der (110)-Pol und bei der Anderen der (112)-Pol im Zentrum. Ein in beiden Fällen gleichgroßer Bereich in einem Radius von $5 \mathrm{~nm}$ um den Pol wurde selektiert und der durchschnittliche Abstand $\overline{\Delta z}$ zur idealen Kugelkappe für diesen Bereich während der Feldverdampfungssimulation bestimmt. Gemäß Gl. 3.1 gilt nun für die Krümmungsradien an den

\footnotetext{
${ }^{1}$ Zwar könnte formal nach Gl. 7.7 auch direkt aus den bekannten $U_{3^{+}}$die benötigte Feldverdampfungsfeldstärke errechnet werden. Diese wäre aber nicht korrekt, da die anliegende Feldstärke die Feldverdampfungsfeldstärke ist
} 

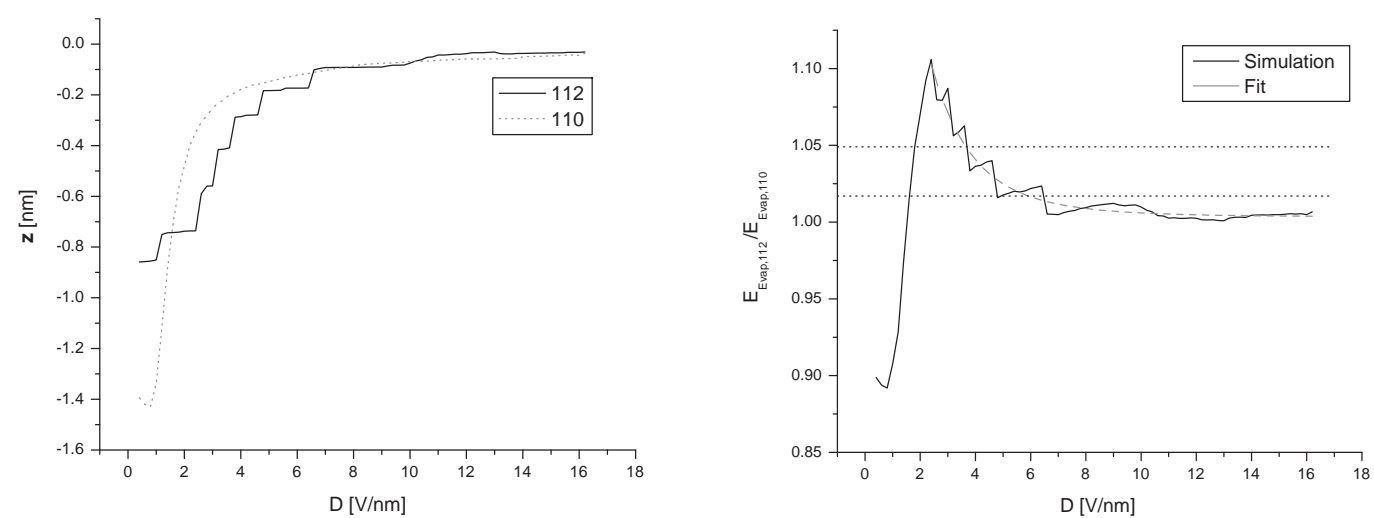

Abbildung 7.6: Der Einfluss von D auf $\overline{\Delta z}$ für die [112] und die [110]-Richtung. In (a) erkennt man, dass sich beide Kurven annähern. Die Kurve der [110]-Richtung zeigt Stufen an den Stellen, an denen eine neue Ebene in den Bereich wandert, innerhalb dessen $\overline{\Delta z}$ bestimmt wurde. Die (112)-Ebenenabstände sind klein genug, damit hier ein solcher Effekt nicht auffällt. In (b) ist das nach $G$. $\%$.10 berechnete Verhältnis $E_{\text {Evap,112 }} / E_{\text {Evap,110 }}$ aufgetragen. Die horizontalen Linien kennzeichnen den experimentell erwarteten Wert (Tabelle 6.1) und dessen $2 \sigma$-Fehlerbalken. Somit lässt sich ein Bereich identifizieren, innerhalb dessen D liegen sollte. Der Fit eines exponentiellen Abfalls ist hierbei hilfreich.

Polen

$$
\frac{E_{E v a p, 1}}{E_{E v a p, 2}}=\frac{R_{2}}{R_{1}}
$$

Nun ist zu überlegen, wie sich $\Delta z$ auf die Radien auswirkt. Offenbar bedeutet ein $\Delta z>0$, also eine aus der idealen Kugelkappe mit Radius $R_{i d}$ herausragende Stelle, einen kleineren Radius. Ein $\Delta z<0$, also eine abgesenkte Stelle der Spitze, verursacht einen größeren Radius. Der genaue Zusammenhang ist nicht trivial, da man alleine aus $\Delta z$ die Krümmung nicht ablesen kann. Der analysierte Bereich wurde zwar so klein gewählt, dass die Werte von $\Delta z$ in ihm nicht stark abweichen. Allerdings gibt dies keine Kenntnis darüber, welche Geometrie abseits dieser Stelle vorhanden ist. Da sich die Spitzenradien nur um einen sehr geringen Wert, nämlich $3 \%$ unterscheiden sollen, kann man allerdings eine lineare Veränderung annehmen, so dass gilt $R_{1} \approx$ $R_{i d}+\overline{\Delta z_{1}}$ und $R_{2} \approx R_{i d}+\overline{\Delta z_{2}}$. Demzufolge gilt

$$
\frac{E_{E v a p, 1}}{E_{E v a p, 2}} \approx \frac{R_{i d}+\overline{\Delta z_{2}}}{R_{i d}+\overline{\Delta z_{1}}}
$$

Man sieht, dass der Wert von $D$ zwischen $3.7 \mathrm{eV} / \mathrm{nm}$ und $6.2 \mathrm{eV} / \mathrm{nm}$ liegen sollte. Mithilfe des Fits wird $D=4.7 \mathrm{eV} / \mathrm{nm}$ identifiziert. Vergleicht man dies mit dem in [Boll2005] verwendeten Wert von $7 \mathrm{eV} / \mathrm{nm}$, der aus der potentiellen Energie eines 
Nb-Atoms über einer Jellium-Oberfläche von Kreuzer et al. [Kreu1992] berechnet wurde, so ist dieser Wert niedriger, aber in der gleichen Größenordnung.

Um den ermittelten Wert von D für andere Simulationen weiterverwenden zu können, muss berücksichtigt werden, dass $\mathrm{W}$ ein sehr hohes $E_{E v a p, W}=57 \mathrm{~V} / \mathrm{nm}$ besitzt. Deshalb sollte eine Normierung stattfinden, bevor $D$ für andere Materialien verwendet wird.

\subsection{Simulationsergebnisse für $\mathrm{Cu}_{3} \mathrm{Au}$}

\subsubsection{Simulationsparameter}

Die vorhergesagten Feldverdampfungsfeldstärken können durch das Müller-SchottkyModell (Gl. 7.7) berechnet werden. Dadurch kann auch ein Ladungszustand des evaporierten Atoms vorhergesagt werden. Die Simulation wurde unter der Annahme, dass Feldverdampfung über eine Ionisation 2. Grades stattfindet, durchgeführt. Somit ist $I_{1}+I_{2}-2 * \Phi=21.1 \mathrm{eV}$ für Au und $18.8 \mathrm{eV}$ für $\mathrm{Cu}$. Problematisch an dieser Festsetzung ist, dass in den Experimenten, besonders für nichtgeordnete Richtungen, ein großer Anteil einfach geladener $\mathrm{Cu}$-Atome gemessen wurde. Nimmt man diesen Ladungszustand als Grundlage, so wäre $I_{1}-\Phi=3.1 \mathrm{eV}$. Zudem wäre eine Betrachtung der Feldverdampfung über die Energien nicht mehr möglich, da dies einen einheitlichen Ionisationsgrad voraussetzt. Da der zweifache Ionisationsgrad in geordnete Richtungen höher ist, nehmen wir diesen in unserer Simulation an. Ohnehin ist dieser Ladungszustand der einzig sinnvoll simulierbare. Für einfach geladenen $\mathrm{Cu}$-Atome würde eine sofortige Evaporation stattfinden, sobald es an der Oberfläche nicht von Au-Atomen festgehalten wird, da ihr $E_{E v a p, C u^{+}}=30 \mathrm{~V} / \mathrm{nm}$ nach [Mill1996] wesentlich geringer sein sollte als $E_{E v a p, C u^{2+}}=43 \mathrm{~V} / \mathrm{nm}$. Dies verursacht einen geringen Peakabstand. Die zweifach geladenen Atome sollten bei einem größeren Peakabstand vorliegen. In den Experimenten ist in der Tat der reine $\mathrm{Cu}$-Peak für geordnete Richtungen sehr klein. Möglicherweise wird er von dem Anteil der zweifachionisieren $\mathrm{Cu}$-Atome verursacht. Die Peakhöhe erscheint dafür allerdings unverhältnismäßig klein. Evtl. liegt die Ursache darin, dass Postionisation [Hayd1980] stattfindet.

Da keine Paarpotentiale in der Literatur vorlagen, wurden die Bindungsenergien aus den Sublimationsenergien (3.81 eV für Au und $3.49 \mathrm{eV}$ für $\mathrm{Cu}$ ) errechnet. Dies 
ergibt, da beide Materialien als kfz, also mit $12 \mathrm{NN}$, auftreten $\Lambda_{\mathrm{Cu}-\mathrm{Cu}}=0.291 \mathrm{eV}$ und $\Lambda_{A u-A u}=0.3175 \mathrm{eV}$

Auch im Falle von $\mathrm{Cu}_{3} \mathrm{Au}$ muss überlegt werden, bis zu welchem Nachbarn die Bindungen berechnet werden sollen. In Tabelle 7.1 ist der Einfluss der Nachbarn verschiedenen Grades auf die Bindungsenergie aufgetragen. Hierbei fällt auf, dass der Einfluss der 3.NN bedeutender ist als der der 2.NN. Für die Berechnungen wurden die Atome bis zum 5.NN miteinbezogen ${ }^{2}$. Die Bindungsenergien müssen also mit dem Faktor 1.325 normiert werden. Die weiter entfernten Nachbarn sind in einem kfzbasierten Gitter somit weniger bedeutend als in einem krz-basiertem, das einen Faktor von 1.774 erfordert. Somit sind $\Lambda_{1 . N N, C u-C u}=0.220 \mathrm{eV}$ und $\Lambda_{1 . N N, A u-A u}=0.240$ eV. Die Gitterparameter von $\mathrm{Cu}$ mit $0.361 \mathrm{~nm}$ und $\mathrm{Au}$ mit $0.408 \mathrm{~nm}$ weichen von den $0.374 \mathrm{~nm}$ der $\mathrm{Cu}_{3} \mathrm{Au}$-Phase ab. Die NN-Abstände, die in den Simulationen verwendet wurden, sind deswegen mit $d_{N N, C u-C u}=0.255 \mathrm{~nm}, d_{N N, C u-A u}=0.264 \mathrm{~nm}$ und $d_{N N, A u-A u}=0.288 \mathrm{~nm}$ für verschiedenen Potentiale unterschiedlich. Dies war bei der Simulation von $\mathrm{Fe}_{3} \mathrm{Al}$ durch T.Kresse [Kres2009] ebenfalls nötig. Es wirkt sich, wie man in Abb.7.7 erkennen kann, besonders auf die Bindung von Gold abschwächend aus, bei dem die Bindungsenergie der $\mathrm{NN}$ im $\mathrm{L1}_{2}$-Gitter nur noch $54 \%$ der Bindung im Minimum beträgt. Nach der Müller-Schottky-Formel wird dadurch auch die Feldverdampfungsfeldstärke herabgesetzt. Zudem kann sich der Grad der erwarteten Ionisation ändern. Auch die Kupferbindungen werden schwächer.

Der Straffaktor $D$ kann durch eine Normierung des für Wolfram bestimmten $D$ errechnet werden, da $U \sim \sqrt{E_{E v a p}}$. Aus den experimentell gemessenen Feldverdampfungsfeldstärken $E_{E v a p, C u_{3} A u} \beta=220(20) \mathrm{V} / \mathrm{nm}$ (für die $<110>$-Richtungen-Diese liefern auch den maximalen Wert) und $E_{\text {Evap }, W} \beta=300(10) \mathrm{V} / \mathrm{nm}$ lässt sich der Straffaktor $D_{\mathrm{Cu}_{3} \mathrm{Au}}=4.7 \sqrt{22 / 30} \mathrm{eV} / \mathrm{nm}=4 \mathrm{eV} / \mathrm{nm}$ bestimmen. Es ist sinnvoll den maximalen Wert für $E_{\text {Evap }} \beta$ zu verwenden, damit auch für diese Richtung eine sinnvolle Simulation möglich ist. Die maximale Anzahl NN für eine Verdampfung wird auf 8 festgesetzt. D.h. in einer schwach gebundenen Ebene ist es prinzipiell sogar möglich vor der über ihr liegenden Ebene zu verdampfen. Dies wird gestattet, da im FIM nie eine der Zwischenebenen in geordnete Richtungen zu sehen war. Zudem muss ohnehin eine genaue Einstellung der Bindungsenergie zumindest für die [100]-Richtung möglich sein, wenn die experimentellen Ergebnisse reproduziert werden sollen.

\footnotetext{
${ }^{2}$ Berücksichtigt man die Nachbarn nur bis zum Abstand der 2.NN, so lässt sich die experimentelle Situation nicht reproduzieren.
} 


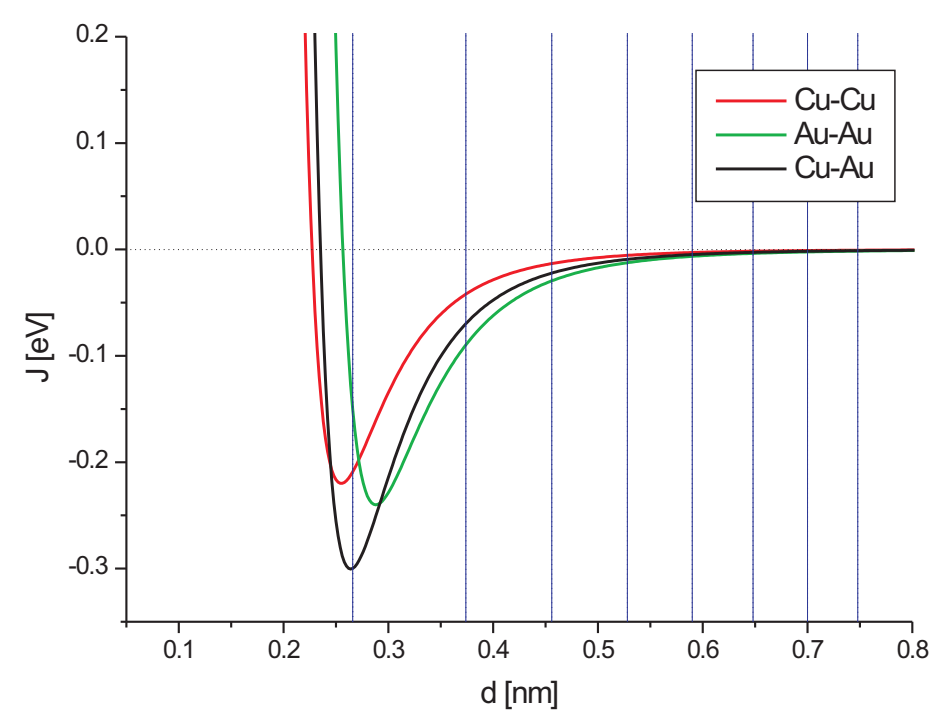

Abbildung 7.7: Lennard-Jones-Potential der Cu-Au-Legierung. Als vertikale Linien sind die Positionen der NN eingezeichnet. Die Cu-Au-Kurve ist entsprechend der Ergebnisse der DFT-Berechnungen aus Abschnitt 7.2.6 eingefügt.

\subsubsection{Bestimmung der $\mathrm{Cu}-\mathrm{Au}-$ Bindungsenergie}

Da die Bindungsenergie gemischter Partner kein experimenteller Wert ist, sondern mit DFT bestimmt wurde, kann man sie auch als variable Größe ansehen, die geändert werden muss um die Simulation der Realität anzupassen. An dieser Stelle zahlt es sich aus, dass die Lennard-Jones-Potentiale sich durch lediglich 2 Parameter beschreiben lassen. Dadurch ist die Bindungsenergie höherer Nachbarn aus der Bindungsenergie der 1.NN einfach zu berechnen, was bei komplizierteren Potentialen nicht mehr möglich wäre. Die Position der 1.NN ist aus dem Gitterparameter bekannt als $0.374 / \sqrt{2} \mathrm{~nm}=0.2644 \mathrm{~nm}$. Berechnet man nun für $\mathrm{Cu}_{3} \mathrm{Au}$ TAP-Daten für eine $<110>$-Richtung, so erhält man AtomVicinity-Dichteprofile, wie sie in Abb. $7.8 \mathrm{zu}$ sehen sind. Für die $<100>$-Richtung erhält man vergleichbare Ergebnisse. Bei $0.22 \mathrm{eV}$ befindet sich ein reiner Cu-Peak links des gemischten Peaks, der sich bei Erhöhung der Bindungsenergie nach rechts auf den gemischten Peak zu bewegt.

Da sowohl von der $<110>$ als auch von der $<100>$-Richtung experimentelle Messungen verfügbar sind (Kap. 6.9) ist es naheliegend, die Bindungsenergie als richtig anzusehen, die diese Messungen am besten reproduziert. Dafür wurde in Abb. 7.9 der Abstand des reinen $\mathrm{Cu}$-Peaks zum rechts von ihm gelegenen Au-Peak angegeben. Man erkennt, dass sich der Abstand beginnend bei $0.25 \mathrm{eV}$ für beide Richtungen Null annähert. Dabei gibt es ein lokales Maximum für die $<100>$-Richtung 


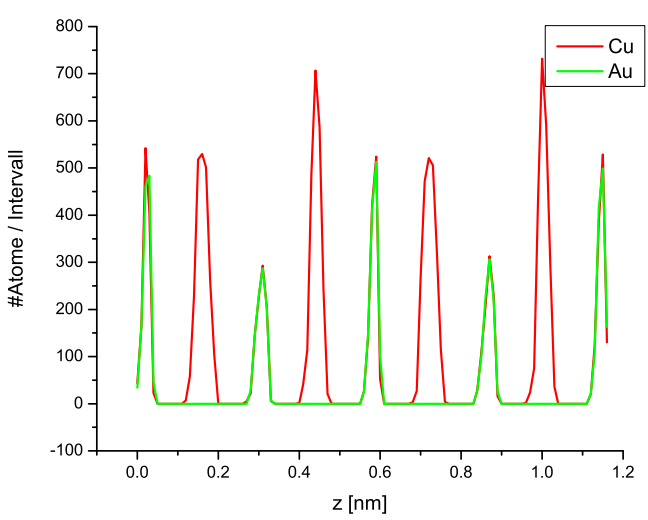

(a) $\Lambda_{1 . N N}=0.13 \mathrm{eV}$

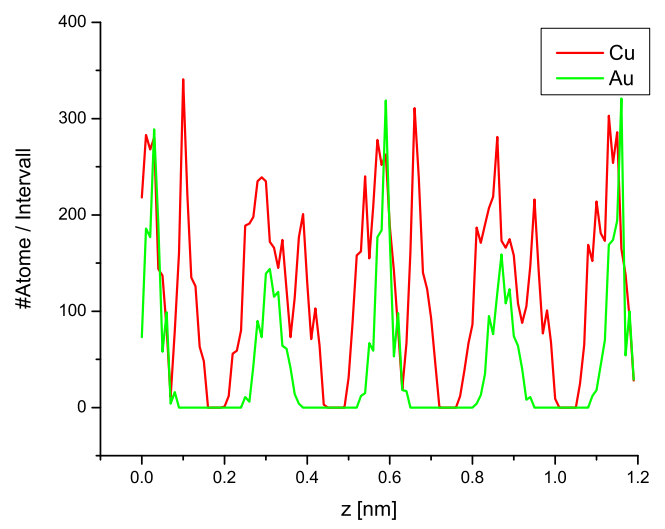

(b) $\Lambda_{1 . N N}=0.19 \mathrm{eV}$

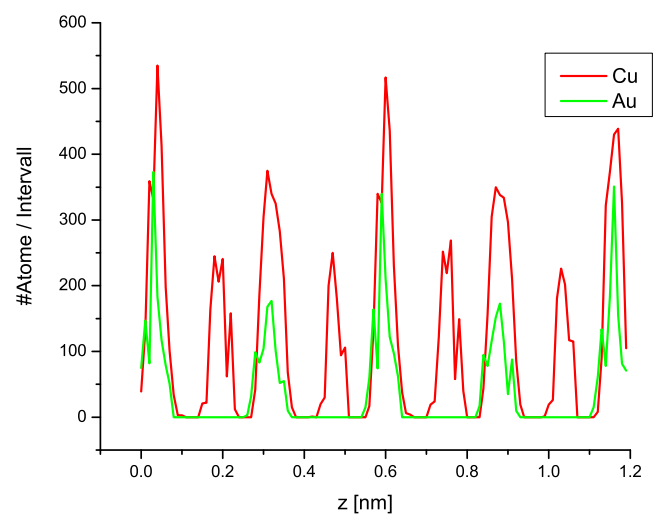

(c) $\Lambda_{1 . N N}=0.22 \mathrm{eV}$

Abbildung 7.8: Simulation von $C u_{3} A u$ für verschiedene $C u$-Au Bindungen in $\langle 110\rangle-$ Richtungen. Es sind die AtomVicinity-Dichteprofile mit Au als Referenzatom dargestellt. Die Bindungesenergien $\Lambda_{1 . N N}$ sind jeweils angegeben. Man erkennt, dass sich in (a) die Au-Peaks mit Cu-Peaks der gleichen Höhe gut überlappen, was bei gemischten Ebenen zu erwarten ist. Rechts und links dieser Peaks befinden sich hohe Cu-Peaks die der reinen Cu-Ebene entsprechen. Es gibt zudem eine minimale Verschiebung des kleinen Cu-Peaks nach rechts, was aus einem geringeren $U_{2}$ für diese Atome folgt. Mit wachsendem $\Lambda_{1 . N N}$ verschieben sich die Peaks $(b, c)$. Der kleine Au-Peak befindet sich nun rechts des kleinen Cu-Peaks, was bedeutet, dass nun $U_{2}$ von Au geringer als $U_{2}$ von $C u$ ist. In (c) ist mit $\Lambda_{1 . N N}=0.22 \mathrm{eV}$ der Au-Peak nahe der Position des großen Cu-Peaks. Bei weiter wachsendem $\Lambda_{1 . N N}$ verringert sich der Abstand zwischen den $C u$-Ebenen wieder, zudem bewegt sich der Au-Peak in Richtung des kleinen Cu-Peaks. 


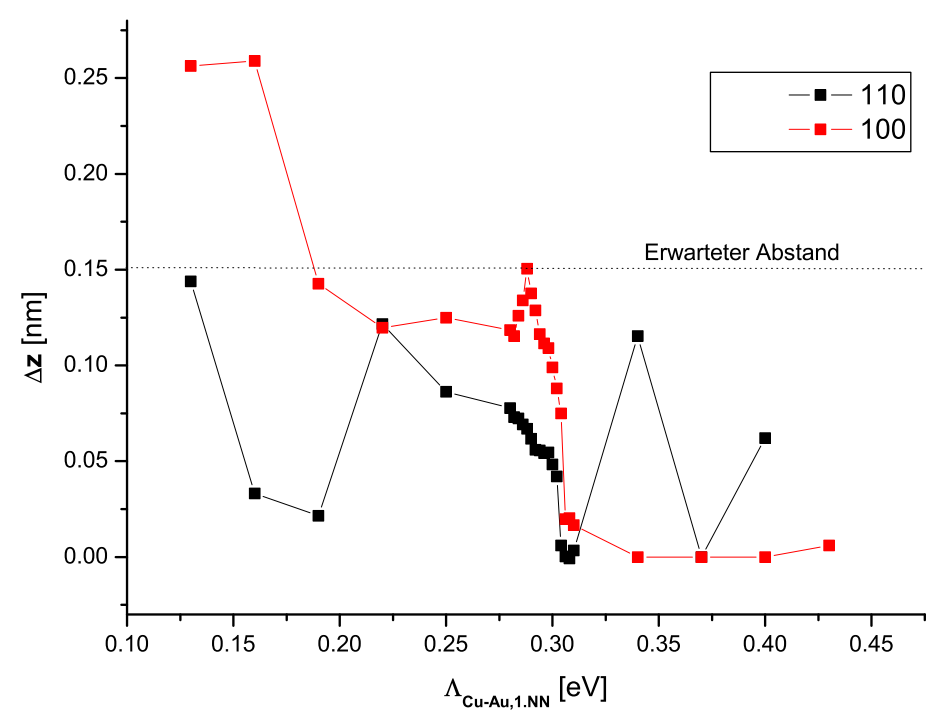

Abbildung 7.9: Der Peakabstand des reinen Cu-Peaks zum rechts von ihm gelegenen AuPeak in Abhängigkeit der NN-Bindungsenergie $\Lambda_{C u-A u, 1 . N N}$ für verschiedene Richtungen. Der experimentell erwartete Peakabstand für die $<100>-$ Richtung ist als horizontale Linie eingezeichnet. Der am stärksten gebundene Partner der $<110>$-Richtung wechselt mehrfach und bewirkt eine Veränderung der Peakbstände.

bei $0.288 \mathrm{eV}$, während der Abstand für die $<110>$-Richtung zunächst stetig abfällt. Da nur für den (100)-Pol ein reiner Cu-Peak ausgemacht werden konnte, kann eine genaue Reproduktion des experimentellen Abstands nur für diese Richtung stattfinden. Der Abstand von $0.15 \mathrm{~nm}$ wird bei $0.288 \mathrm{eV}$ erreicht. Dieses Resultat stimmt gut mit den Ergebnissen der DFT aus Abschnitt 7.2.6 überein, welches $\Lambda_{1 . N N, C u-A u}-\left(\Lambda_{1 . N N, A u-A u}+\Lambda_{1 . N N, C u-C u}\right) / 2=0.10 \mathrm{eV}$ also $\Lambda_{C u-A u}=0.27 \mathrm{eV}$ (für die Potentiale an der Stelle der $1 . \mathrm{NN}$ in der $\mathrm{L} 1_{0^{-}}$Struktur) vorhersagt.

Ein weiteres Kriterium, um die Korrektheit des Wertes abzuschätzen, ist die Unterscheidbarkeit der Ebenenpeaks. Die ist für die $<110>$-Richtung in keiner der Messungen gegeben gewesen. Die Bindung muss demzufolge mindestens so stark sein, dass gemischte und reinen Ebenen nicht unterscheidbar sind . Dies wäre bei einfachem Ablesen aus dem Diagramm bei $0.302 \mathrm{eV}$ der Fall. Dies wäre nicht konsistent mit dem ermittelten Wert da $0.302 \mathrm{eV}>0.288 \mathrm{eV}$ ist. Die Ursache dieser Abweichung liegt darin begründet, dass die Begrenzung der Auflösung durch das Verrauschen der Messung in der Simulation nicht berücksichtigt wird. Um dies zu tun wird die Halbwertsbreite des Au-Peaks in der Simulation von $0.023 \mathrm{~nm}$ mit der Halbwertsbreite, die typischerweise im Experiment für die $<110>$.-Richtung ermittelt wurde, von 0.05 verglichen. Die Halbwertsbreite der $\mathrm{Cu}$-Peaks kann höchstens 


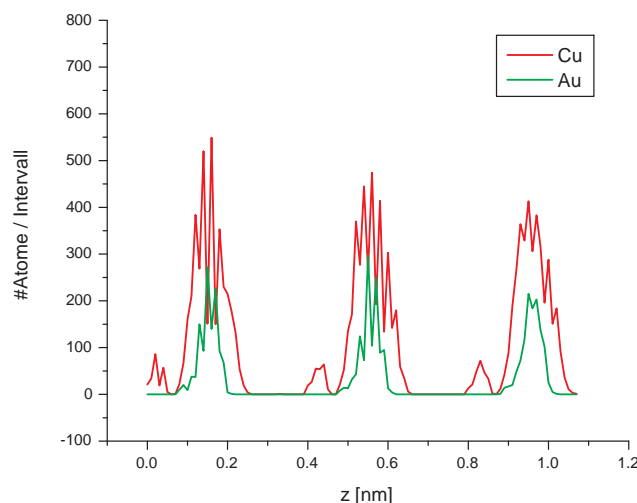

(a) $<100>$

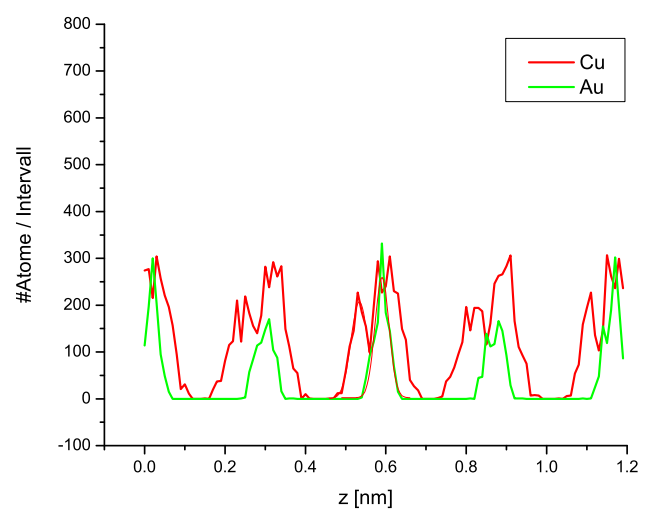

(b) $<110>$

Abbildung 7.10: AtomVicininity-Dichteprofile (Referenzatom: Au) von Cu $u_{3}$ A u simuliert für die $<100>$ und $<110>$-Richtung.

größer sein, solange Au das Referenzatom ist. In Abb. 7.8(a) erkennt man, dass sie im optimalen Fall genauso groß ist wie von Au. Es ist also vernünftig, anzunehmen, dass die Halbwertsbreite in Experiment und Simulation für $\mathrm{Cu}$ - und $\mathrm{Au}-\mathrm{Peaks}$ gleichartig skaliert ist. Die Halbwertsbreite, die man experimentell erwarten würde liegt somit bei $(0.05 / 0.023) * 0.42 \mathrm{~nm}=0.8 \mathrm{~nm}$. Die erwartete minimale Bindung für $\mathrm{Cu}$ mit $\mathrm{Au}$ ist also durch den Verlauf der Lennard-Jones-Funktion mit $\Lambda_{1 . N N}=0.25$ $\mathrm{eV}$ gegeben.

Mit dem gleichen Argument kann man eine obere Grenze angeben. Die Ebenen müssen für die $<100>$-Richtung noch trennbar sein. Die Halbwertsbreite von Au ist im Experiment nicht viel größer als in der Simulation. Weswegen keine Skalierung stattfinden sollte (Dies entspricht einer etwas vorsichtigeren Schätzung als eigentlich nötig wäre). Der Maximalwert der NN-Bindung im Cu-Au-Potential ist also $\Lambda_{1 . N N}=$ $0.304 \mathrm{eV}$.

In Abb. 7.10 sind die AtomVicinity-Dichteprofile für den ermittelten Wert der CuAu-NN-Bindungsenergie $\Lambda_{1 . N N, C u-A u}=0.288 \mathrm{eV}$ aufgetragen. Für die $<100>$-Richtung stimmt die Peakabfolge mit einem kleinen $\mathrm{Cu}$-Peak, der von einem hohen $\mathrm{Au}-$ und anschließend einem hohen Cu-Peak gefolgt wird. Für die $<110>$-Richtung ist dies allerdings nicht der Fall. Die Peakabfolge, die nach experimentellen Ergebnissen $\mathrm{Au}-\mathrm{Cu}$ sein müsste, ist nicht gegeben. Eine Erklärung hierfür ist, dass in der Messung im dem Au-Peak nachfolgenden Peak hohe Anteile der einfach geladenen $\mathrm{Cu}$-Ionen gemessen wurden. Die Simulation berücksichtigt nicht, dass diese Ionen eine niedrigere Feldverdampfungsfeldstärke haben müssten. Die reine Ebene hätte 


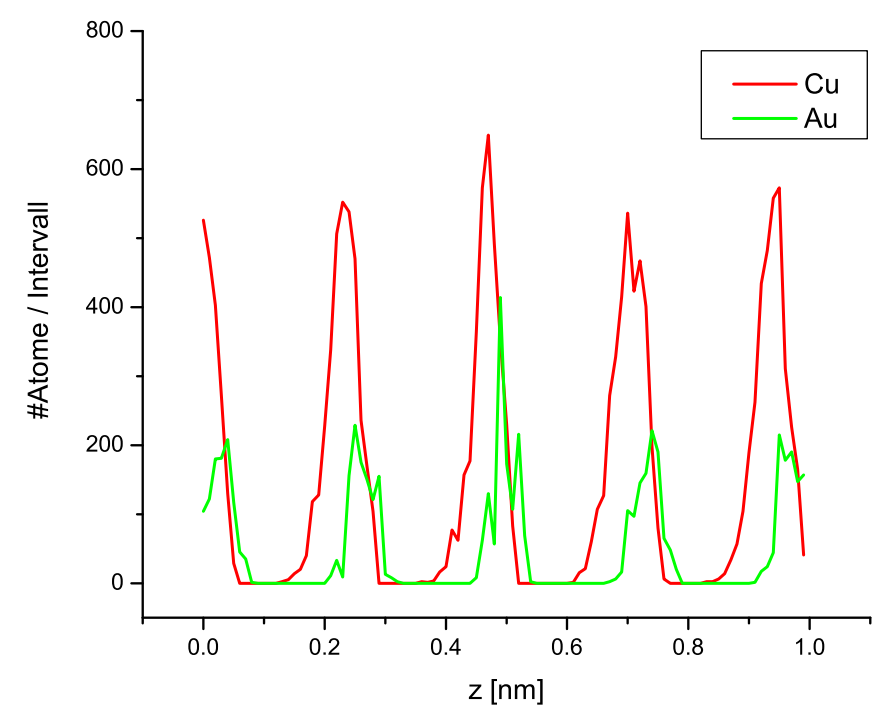

Abbildung 7.11: AtomVicinity-Dichteprofile für simuliertes $C u_{3} A u$ in $<111>$-Richtung. Die Au-Peak sind gegenüber den Cu-Peaks um 0.027(2) nm nach rechts verschoben.

also eine geringere Feldverdampfungsfeldstärke als von der Simulation vorausgesagt. Dies würde den Abstand zum Au-Peak erhöhen und kann dadurch die veränderte Peakreihenfolge im Experiment verursachen.

Um die Plausibilität der Simulation weiter zu testen ist in Abb. 7.11 das Ergebnis für die nicht geordnete $<111>$-Richtung dargestellt. Die Peakabfolge ist, wie experimentell nach Kap. 6.8.2 erwartet wird, Cu-Au. Sogar quantitativ ergibt sich für die Verschiebung der $\mathrm{Au}$ - gegenüber den $\mathrm{Cu}$-Peaks eine sehr gute Übereinstimmung. Die Verschiebung um 0.027(2) nm in der Simulation steht einer experimentell bestimmten Verschiebung von 0.026(2) gegenüber. Dies ist nicht nur eine gute Bestätigung für die Korrektheit der $\mathrm{Cu}$-Au-Bindung, sondern auch ein starker Hinweis, dass andere Parameter, insbesondere der Straffaktor $D$, richtig gewählt sind.

\subsection{Simulationsergebnisse für $\mathrm{TiAl}+\mathrm{X}$}

\subsubsection{Simulationsparameter}

Die nach dem Müller-Schottky-Modell vorhergesagten Ionisationszustände weichen für einige der verwendeten Elemente in der geordneten TiAl-Phase von den experimentell ermittelten Werten ab (Kap. 6.8.1). Alle Elemente außer Ag werden nahezu 
ausschließlich zweifach ionisiert detektiert. Al, Ag und $\mathrm{Cr}$ sollten eigentlich einfach geladen auftauchen. Al müsste eine geringes $E_{\text {Evap }}=19 \mathrm{~V} / \mathrm{nm}$ besitzen. Zweifach geladenes Aluminium würde $33 \mathrm{~V} / \mathrm{nm}$ besitzen. Letzteres stimmt wesentlich besser mit den Abschätzungen der Feldverdampfungsfeldstärken in Kap. 6.8.1 überein. Dies spricht dagegen, dass der zweifache Ladungszustand erst durch Postionisation eintritt, weswegen die Voraussetzung für unsere Simulation, dass $E_{\text {Evap }}$ sich für alle Elemente aus einem identischen Ionsiationsgrad $(n=2)$ berechnet, als gültig betrachtet werden kann. Das einzige Element, das zu großen Teilen einfach geladen auftritt, ist Ag. Eigentlich wäre es deswegen für diesen Fall nötig, die Feldverdampfungsfeldstärken zu vergleichen. Da diese aufwändige Berechnung jedoch umgangen werden soll, wird stattdessen nach Gl. 7.7 ein Parameter $I_{1}+I_{2}-2 \Phi=13.7 \mathrm{eV}$ konstruiert, der die für einfach geladenes Ag erwartete Feldverdampfungsfeldstärke von $24 \mathrm{~V} / \mathrm{nm}$ ergibt.

\begin{tabular}{|l|l|lll|c|}
\hline Element & $\Lambda_{S u b}$ & $I_{1}[\mathrm{eV}]$ & $I_{2}[\mathrm{eV}]$ & $\Phi[\mathrm{eV}]$ & $I_{1}+I_{2}-2 \Phi[\mathrm{eV}]$ \\
\hline Al (kfz) & 3.34 & 5.99 & 18.83 & 4.1 & 16.6 \\
Ti (hdp,krz ab 1000K) & 4.855 & 6.82 & 13.58 & 4.0 & 12.4 \\
Nb (krz) & 7.47 & 6.88 & 14.32 & 4.0 & 13.2 \\
Ag (kfz) & 2.96 & 7.576 & 21.49 & 4.6 & 19.9 (13.7 korrigiert) \\
Cr (krz) & 4.10 & 6.766 & 16.50 & 4.6 & 14.1 \\
\hline
\end{tabular}

Tabelle 7.7: Sublimationsenergien $\Lambda_{\text {Sub }}$, Ionisationsenergien I und Austrittsarbeiten $\Phi$ für die reinen Elemente [Mill1996]. Die Gitterstruktur kfz (kubischflächenzentriert), krz (kubischraumzentriert) und hdp (hexagonal dichteste Packung) ist jeweils mitangegeben. Für Silber ist in Klammern die Energie angegeben, die vorliegen müsste, wenn die Feldverdampfungsfeldstärke gleich der für einfach geladenes Ag wäre.

\subsubsection{TiAl-Bindungspotentiale}

Die Angabe der Potentiale ist hier für reines $\mathrm{TiAl}$ angenehmer als für $\mathrm{Cu}_{3} \mathrm{Au}$, da die NN-Abstände in Ti und Al mit $d_{N N, T i}=0.293 \mathrm{~nm}$ sowie $d_{N N, A l}=0.286 \mathrm{~nm}$ ähnlich sind. Betrachtet man die krz-Phase von Ti, so ist der NN-Abstand dort mit 0.286 sogar identisch. Die Verhältnisse im L1 $1_{0}$-Mischkristall werden durch die Dehnung der Einheitszelle in $z$-Richtung etwas komplizierter. Es wurde für die Simulation von der mit DFT berechneten Gitterstruktur ausgegangen. Aus diesem Grunde ergibt sich scheinbar das Problem, die Position des Minimums des Lennard-Jones-Potentials für die gemeinsame Bindung festzusetzen. Bei genauerer Betrachtung eines perfekt 
geordneten Systems, erkennt man, dass die 8 gemischten Bindungen immer zwischen zwei Ebenen in [001]-Richtung vorhanden sind. In [010] sowie [100]-Richtung befinden sich nur $4 \mathrm{NN}$ der gleichen Art. Mit $c=0.4057 \mathrm{~nm}$ und $a=0.3989 \mathrm{~nm}$ ergibt sich $d_{N N, A l-T i}=0.5 \cdot \sqrt{a^{2}+c^{2}}=0.284 \mathrm{~nm}$. Die gemischten Bindungsenergien ergeben sich nun aus den DFT-Berechnungen.

Dort wurden allerdings nur die Unterschiede zwischen den verschiedenen Bindungen berechnet. Für die Simulation eines binären Systems ist in der Tat auch nur dies wichtig. Die Erhöhung aller Werte um eine Konstante hat für die Evaporationsreihenfolge keine Bedeutung. Sobald weitere Elemente in der Legierung auftauchen, muss die Bindungsenergie allerdings stimmen. Da der Vergleich der Al-Al und Ti-Ti-Bindungen aus den Sublimationsenergien mit den DFT-Ergebnissen eine plausible Übereinstimmung ergibt, wird von diesen Werten ausgegangen. Demnach ergibt sich für die Bindung von Aluminium, bei einer Normierung, die eine passende Sublimationsenergie bei der Summierung bis zu den 5. NN liefert, $\Lambda_{1 . N N, A l-A l}=3.39 /(12 \cdot 1.325) \mathrm{eV}=0.213 \mathrm{eV}$. Die Berechnung für Ti gestaltet sich etwas schwieriger, da es bei hohen Temperaturen krz mit einer Gitterkonstante von $0.287 \mathrm{~nm}$ auftritt. Nun stellt sich die Frage, wie man aus dieser Bindung auf die Bindung in einer kfz-Struktur schließen kann. Rechnet man mit der krz-Gitterkonstanten als $d_{N N, T i-T i}$, so erhält man mit Normierung für krz-Gitter $\Lambda_{1 . N N, T i-T i}=4.85 /(8 \cdot 1.8) \mathrm{eV}=0.334 \mathrm{eV}$. Nach der DFT-Rechnung (Kap. 7.2.3) erwartet man zu $\Lambda_{1 . N N, A l-A l}$ einen Unterschied von $0.35 \mathrm{eV}$ anstelle der hier ermittelten $0.121 \mathrm{eV}$. Da mit dem größeren Unterschied eine bessere Übereinstimmung mit den Potentialen nach Zope [Zope2003] in Abb. 7.1 erreicht wird (dabei ist berücksichtigt dass Zope verm. nur die Bindungsenergie der 1.NN für die Sublimationsenergie einbezieht), verwenden wir diesen, also $\Lambda_{1 . N N, T i-T i}=0.56 \mathrm{eV}$.

Die NN-Abstände für die Potentiale der hinzulegierten Elemente wurden ebenfalls von den reinen Materialien übernommen. Demnach sind $d_{N N, N b}=0.286 \mathrm{~nm}$, $d_{N N, A g}=0.289 \mathrm{~nm}$ und $d_{N N, C r}=0.249 \mathrm{~nm}$. Für die Bindungsenergien der LennardJones-Potentiale gilt $\Lambda_{1 . N N, N b-N b}=7.47 /(8 \cdot 1.80) \mathrm{eV}=0.519 \mathrm{eV}$ und $\Lambda_{1 . N N, C r-C r}=$ $4.10 /(8 \cdot 1.80) \mathrm{eV}=0.285 \mathrm{eV}$, nach der Normierung für die krz-Struktur auf die ersten $5 \mathrm{NN}$, sowie $\Lambda_{1 . N N, A g-A g}=2.96 /(12 \cdot 1.325) \mathrm{eV}=0.186 \mathrm{eV}$ nach entsprechender Normierung für die kfz-Struktur.

Die Bindungsenergien der gemischten Paare ergeben sich aus den DFT-Berechnungen (Tabelle 7.4). Die Daten der für TiAl $+\mathrm{X}$ verwendeten Lennard-Jones-Potentiale sind in Tabelle 7.8 aufgelistet. Die $d_{N N}$ sind jeweils als der minimale NN-Abstand der 


\begin{tabular}{|l|l|l|}
\hline Bindungspartner A-B & $\Lambda_{N N}[\mathrm{eV}]$ & $d[\mathrm{~nm}]$ \\
\hline $\mathrm{Al}-\mathrm{Al}$ & 0.213 & 0.286 \\
$\mathrm{Ti}-\mathrm{Ti}$ & 0.56 & 0.286 \\
$\mathrm{Nb}-\mathrm{Nb}$ & 0.519 & 0.286 \\
$\mathrm{Ag}-\mathrm{Ag}$ & 0.186 & 0.289 \\
$\mathrm{Cr}-\mathrm{Cr}$ & 0.285 & 0.249 \\
$\mathrm{Al}-\mathrm{Ti}$ & 0.493 & 0.284 \\
$\mathrm{Al}-\mathrm{Nb}$ & 0.503 & 0.284 \\
$\mathrm{Ti}-\mathrm{Nb}$ & 0.652 & 0.284 \\
$\mathrm{Al}-\mathrm{Ag}$ & 0.205 & 0.284 \\
$\mathrm{Ti}-\mathrm{Ag}$ & 0.363 & 0.284 \\
$\mathrm{Al}-\mathrm{Cr}$ & 0.306 & 0.284 \\
$\mathrm{Ti}-\mathrm{Cr}$ & 0.458 & 0.284 \\
$\mathrm{Nb}-\mathrm{Nb}$ (korr.) & 1.20 & 0.286 \\
$\mathrm{Nb}-\mathrm{Al}$ (korr.) & 0.84 & 0.284 \\
$\mathrm{Nb}-\mathrm{Ti}$ (korr.) & 0.90 & 0.284 \\
\hline
\end{tabular}

Tabelle 7.8: Daten der für TiAl $+X$ verwendet Lennard Jones-Potentiale. $d_{N N}$ ist die Position, $\Lambda_{N N}$ die Tiefe des Minimums. Für Nb sind die berechneten, sowie korigierte (korr.) Potentiale angegeben

$\mathrm{L} 1_{0}$-Zelle angenommen. Letztlich ist dies etwas beliebig. Da sich die NN-Abstände aller Materialien (außer Chrom) allerdings kaum unterscheiden und ein Unterschied von $0.005 \mathrm{~nm}$ kaum einen Einfluss auf das Potential hat, wird dieser Wert zum besseren Vergleich mit dem Ti-Al-Potential gewählt.

Der Parameter $D$ für die Strafenergie muss für TiAl erneut angepasst werden. Die Feldverdampfungsfeldstärke von TiAl liegt zwar für die nichtgeordnete [111]Richtung bei etwa $25 \mathrm{~V} / \mathrm{nm}$ (Tabelle 6.3). Für geordnete Richtungen werden allerdings höhere Werte gemessen. Sie liegen für die [110]-Richtung bei $E_{E v a p, C u_{3} A u} \beta=$ $270(20) \mathrm{V} / \mathrm{nm}$. Daraus ergibt sich mit $E_{\text {Evap }, W} \beta=300(10) \mathrm{V} / \mathrm{nm}$ ein Straffaktor $D=4.7 \sqrt{27 / 30} \mathrm{~V} / \mathrm{nm}=4.4 \mathrm{~V} / \mathrm{nm}$. Da in den FIM-Bildern häufig ein dunklerer Rand unter der hellen, abdeckenden Ebene zu sehen war, wird die maximale Anzahl der 1.NN, bei der ein Atom verdampfen darf, auf 6 festgelegt. Eine schwach gebundenen Ebene kann also eine stark gebundene Ebene nicht mehr überholen.

Bei den später im Abschnitt 7.6.5 für die nichtgeordnete [100]-Richtung dargestellten Simulationen fällt auf, dass sich die experimentell bestimmten Peakreihenfolgen für TiAl mit Nb-Zusätzen nicht reproduzieren lassen. Dies kann nur durch eine ex- 


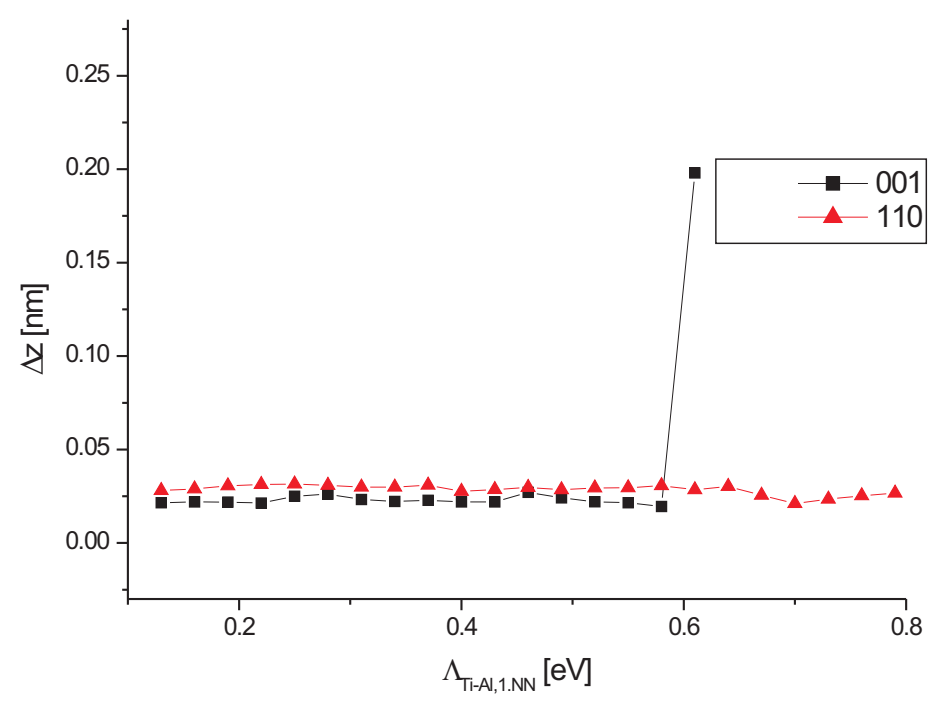

Abbildung 7.12: Ti-Al-Peakabstand in den AtomVicinity-Dichteprofilen in Abhängigkeit von $\Lambda_{1 . N N, A l-T i}$. Das Verhalten für die [001]-Richtung ändert sich bei 0.58 eV plötzlich. Im Grunde findet an dieser Stelle eine Aufspaltung der Peaks statt. Der Abstand des kleineren Anteils ist eingezeichnet. Der Hauptpeak verschiebt sich nicht. Für höhere Energien verändern sich die Abstände ständig, erreichen allerdings nie wieder Ti-Al-Peakabstände von 0.02 nm zueinander. Das Verhalten für die [110]-Richtung verändert sich erst bei wesentlich höheren Energien.

treme Veränderung der Parameter erreicht werden. Es erscheint allerdings durchaus gerechtfertigt, solch eine Veränderung vorzunehmen. Da Nb mit Al und mit Ti im Vergleich mit den anderen Zusätzen sehr viel stärkere A-B-Bindungen bildet (Kap. 7.2.5), ist auch eine starke Veränderung des Nb-Nb-Paarpotentials denkbar. Nimmt man für $\Lambda_{N b-N b}=1.20 \mathrm{eV}$ an, so ergeben sich die in Tabelle 7.8 eingetragenen alternativen Bindungsenergien.

\subsubsection{Abschätzung der Ti-Al-Bindungsenergie}

Im Gegensatz zu $\mathrm{Cu}_{3} \mathrm{Au}$ ist die Unterscheidung der experimentell ermittelten AtomVicinity-Ebenenpeaks für alle Überstrukturrichtungen ähnlich gut (bzw. schlecht) möglich. Es gibt zwar eine Variation der Peakpositionen, die allerdings mit dem Ordnungsgrad zusammenhängt und nicht mit der kristallographischen Richtung. Somit kann im Gegensatz zu Cu3Au kein genauer Wert für die A-B-Bindungsenergie angegeben werden. Zumindest nach unten lässt sich die Ti-Al-Bindungsenergie allerdings abschätzen, da die Bindung mindestens so stark sein muss, dass in keine der Überstrukturrichtungen Ebenen unterschieden werden können. In Abb. 7.12 


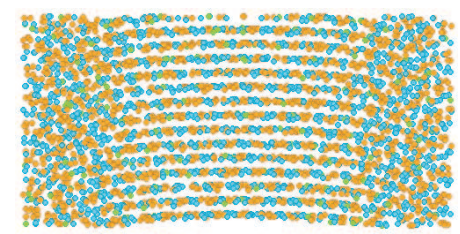

Abbildung 7.13: Simulation von TiAl-Atomsondendaten in [100]-Richtung. In z-Richtung liegen gemischte $\mathrm{Al} / \mathrm{Ti}$-Ebenen im Abstand von $0.2 \mathrm{~nm}$ vor. In der Mitte sind die Ebenen gut zu unterscheiden, am Rand nicht.

ist die Abhängigkeit des Peakabstand von $\Lambda_{1 . N N, T i-A l}$ angegeben. Demnach muss $\Lambda_{1 . N N, T i-A l}<0.58 \mathrm{eV}$ sein. Der auf den DFT-Rechnungen basierende Wert von 0.493 eV widerspricht den Simulationsergebnissen für geordnete Richtungen also nicht. Der gutmütige Verlauf der Kurven ist hierbei vor allem der Bedingung, dass bei einem evaporierbaren Atom höchsten 6 NN 1. Grades vorhanden sein dürfen, zu verdanken. Bei weniger strengen Anforderungen "überholt" der Ti-Peak den Al-Peak, da eine Ti-Ebene bereits vor der über ihr liegenden Al-Ebene verdampft, was nicht mit den experimentellen Beobachtungen zusammenpasst.

\subsubsection{Auflösbarkeit der Netzebenen}

In Abb. 7.13 ist die Seitenansicht einer simulierten Atomsondenmessung in die nicht geordnete [100]-Richtung von TiAl zu sehen. Man erkennt, dass, abseits des im Zentrum liegenden Pols, die Netzebenen schlechter getrennt werden können. Dies ist ein Effekt des Rekonstruktionsalgorithmus. Da die Atome im Zentrum ebenenweise von außen nach innen abgetragen werden, ist die zeitliche Streuung im Vergleich zu weiter außen liegenden Atomen klein. Da aus der Reihenfolge die z-Komponente bestimmt wird, ist diese in den Aussenbereichen zufälliger verteilt. Dieses Verhalten wird auch experimentell beobachtet (Kap. 6.7).

\subsubsection{Die nichtgeordnete [100]-Richtung}

Die Betrachtung der simulierten AtomVicinity-Dichteprofile in die nichtgeordnete [100]-Richtung in Abb. 7.14 reproduziert im wesentlichen die experimentell erwarteten Peakreihenfolgen (Kap.6.8.1). Es ist dabei besonders zu beachten, ob eine veränderte Platzbesetzung eine Veränderung der Verhältnisse für die Feldverdampfung für die nichtgeordnete Richtung bedeutet. Für das nicht korrigierte Nb sowie 
für Ag ist dies der Fall. Dies ist problematisch, da dadurch keine eindeutige Aussage über die Feldverdampfungsfeldstärken getroffen werden kann, wenn man nur experimentelle Messungen betrachtet.

Für Ag wird diese Verhalten experimentell zudem nicht beobachtet, was allerdings daran liegen kann, dass nur wenige Messungen in die nichtgeordnete [100]-Richtung zur Verfügung standen. Für Nb hingegen standen ausreichend viele Messungen zur Verfügung, die zudem keine der simulierten Peakabfolgen ergeben. Da sich mit den durch die DFT errechneten Bindungspotentialen für Nb die experimentell beobachtete Ebenenreihenfolge (Nb-Al-Ti) nicht reproduzieren lässt, muss die Nb-Nb-Bindung auf $\lambda_{1 . N N, N b-N b}=1.2 \mathrm{eV}$ angepasst werden.

Für Chrom werden experimentell zwei verschiedene Peakabfolgen beobachtet. TiCr-Al und Ti/Cr-Al (Kap. 6.8.1.3). Dies lässt sich dank der Simulationen durch eine unterschiedliche Platzbesetzung erklären. Werden Al-Plätze besetzt, so sind die Peaks besser trennbar als bei einer Substituierung der Ti-Plätze.

Die Standardabweichungen aus der Berechnung (aus den Gaussfits) bewirken Fehlerbalken, die kleiner sind als die Symbole.

\subsubsection{Die Überstrukturrichtungen}

Die Positionen der Ebenenpeaks können auch für Simulationen der geordneten Richtungen ermittelt werden. Wären alle Feldverdampfungsfeldstärken identisch, so müsste in [001]-Richtung zwischen den Ti- und Al-Peaks Abstände von $0.2 \mathrm{~nm}$ bestehen. Dies ist, wie in Abb. 7.15 zu erkennen ist, nicht der Fall. Die Abstände sind wesentlich geringer.

Die Peakabfolgen sind wichtig, um zu überprüfen, ob die experimentell ermittelten Ebenenpeaks des hinzulegierten Elements der richtigen Ti- oder Al-Ebene zugeordnet wurden. Substituiert man Al durch ein anderes Element, so sagen die Simulationen vorher, dass dieses, unabhängig davon welches Element gewählt wurde, vor dem Al-Peak auftritt. Dies wird lediglich bei Ag nicht experimentell beobachtet. Dies liegt daran, dass in den Al-Ebenen liegendes Ag einfach ionisiert wird und somit eine höhere Feldverdampfungsfelstärke besitzt.

Wird Ti substituiert, so sagt die Simulation, im Vergleich zum Abstand Al-Peak zu X-Peak, einen geringeren Abstand voraus. Der Nb-Peak tritt hinter dem Ti-Peak, 


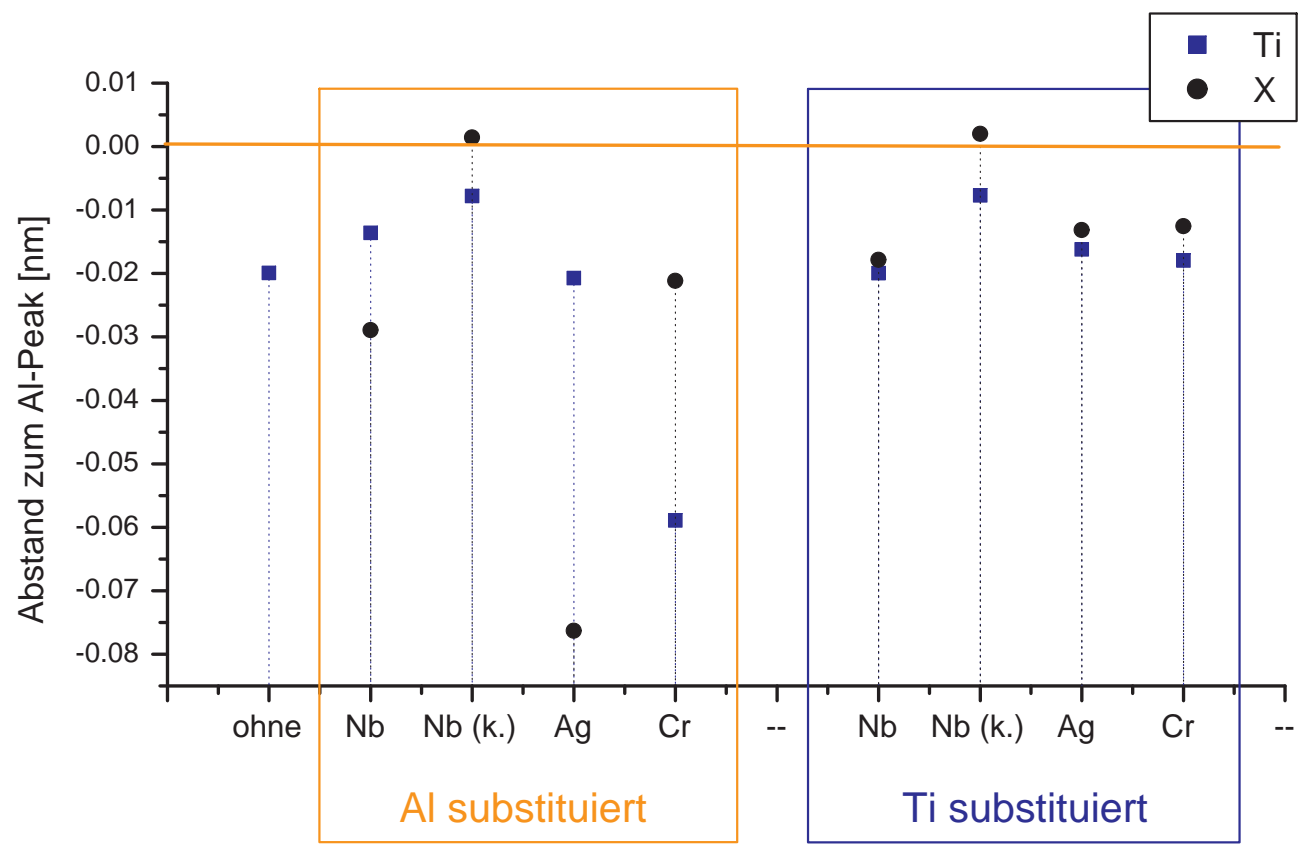

Abbildung 7.14: Peakabstände der Ti- und X-Peaks zu Al aus den AtomVicinityDichteprofilen und Kreuzkorrelation. Es sind jeweils 5 at.\% von $X=\mathrm{Nb}$, Ag, Ar eingeführt, die entweder nur Ti oder nur Al-Atome in der L10-TiAl-Matrix substituieren. Analyserichtung ist die nichtgeordnete [100]-Richtung. Die Position des Al-Peaks ist durch die horizontale orange Linie erkennbar. $\mathrm{Nb}(k$.) bezeichnet die Simulation mit korrigierten Bindungsenergien, die den experimentellen Beobachtungen entsprechen. 


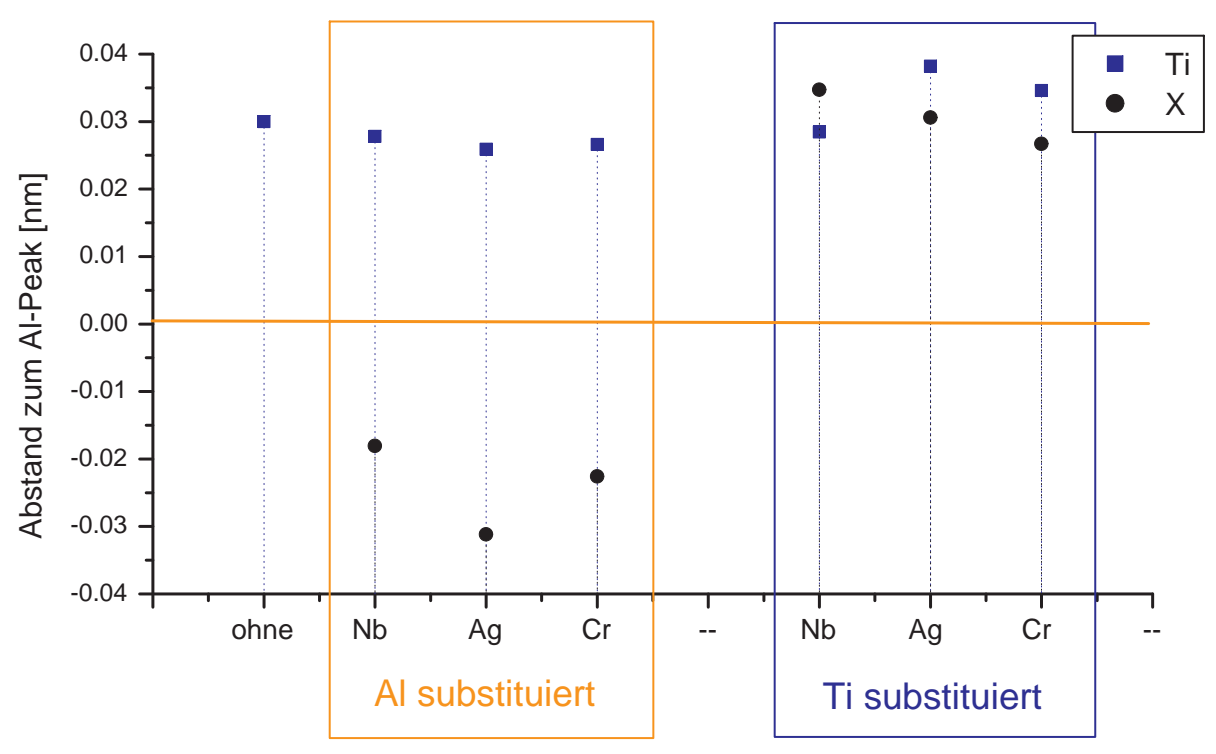

Abbildung 7.15: Peakabstände der Ti- und X-Peaks zu Al aus den AtomVicinityDichteprofilen und Kreuzkorrelation. Es sind jeweils 5 at.\% von $X=N b$, Ag, Ar eingeführt, die entweder nur Ti oder nur Al-Atome in der L1 $1_{0}$-TiAl-Matrix substituieren. Analyserichtung ist die geordnete [001]-Richtung. Die Position des Al-Peaks ist durch die horizontale orange Linie markiert. Die Ergebnisse für $\mathrm{Nb}$ beruhen auf den korrigierten Parametern.

die Ag- und Cr-Peaks vor dem Ti-Peak auf. Für Ag und Nb wird dieses Verhalten experimentell so beobachtet. Im Fall von Cr liegen die 2 Cr-Peaks experimentell in allen Fällen in dem großen Zwischenraum zwischen den Ti- und Al-Peaks 6.10.4. Dies lässt sich nur dadurch erklären, dass Cr tatsächlich hinter dem Ti-Peak auftaucht, falls es Ti substituiert. Ein Cr-Peak liegt dabei immer sehr nahe am Ti-Peak und überlagert sich meist mit ihm. Der Unterschied der Feldverdampfungsfeldstärken von Cr und Ti ist somit klein, wie es von der Simulation vorhergesagt wird. Die unterschiedliche Abfolge ist somit durch eine kleine Abweichung der Chrom-Bindungen in den Simulationen im Vergleich zu den Experimenten erklärbar.

\subsubsection{Einfluss des Ordnungsgrades}

In den experimentellen Ergebnissen für die geordnete Richtung werden stark voneinander abweichende Ti-Al-AtomVicinity-Peakabstände beobachtet. Dies kann durch einen veränderten Ordnungsgrad erklärt werden. Beträgt bei simulierten Atomsondendatn von TiAl in [001]-Richtung der Ti-Al-Ebenenabstand noch $0.3 \mathrm{~nm}$, so ist dieser bei einer Entordnung von 0.1 auf $0.47 \mathrm{~nm}$ und bei einer Entordnung von 
0.2 auf $0.58 \mathrm{~nm}$ angewachsen. Bei geringer Entordnung ist also ein deutlicher Effekt auf die Abstände sichtbar. Auch ein stöchiometrisches Ungleichgewicht kann diesen Effekt ebenfalls erreichen. Ursache für größere Abstände ist die Angleichung der Feldverdampfungsfeldstärken der Ebenen. Durch die Entordnung wird die Feldverdampfungsfeldstärke der nicht mehr reinen Al-Ebene herabgesetzt und die der Ti-Ebene erhöht.

Der Effekt lässt sich auch durch die hinzulegierten Elemente erzeugen. Insbesondere das Substituieren von $\mathrm{Ti}$ mit Nb gleicht die Feldverdampfungsfeldstärken der Ebenen an und führt sogar dazu, dass die gemischte Ti/Nb-Ebene die höchste Feldverdampfungsfeldstärke besitzt [Boll2005].

\subsection{Ausblick}

Es wurde bereits gezeigt, dass Simulationen im Vergleich mit experimentellen Ergebnissen, geeignet sind die A-B-Bindungsenergien in intermetallischen Phasen festzustellen. Im Fall von TiAl mit ternären Elementen war dies nur als Abschätzung und unter Modifikation der mit DFT berechneten Parameter für Nb möglich. Für Cr- und Ag-Zulegierung sowie für die $\mathrm{Cu}_{3} \mathrm{Au}$-Phase waren die Vorhersagen besser. Generell ist die vorgestellt Methode zur Berechnung der Bindungen und ihre Berücksichtigung in Form von Lennard-Jones-Potentialen somit geeignet, Vorhersagen über experimentelle Ergebnisse von TAP-Analysen zu treffen. Insbesondere bei der Interpretation der Peakreihenfolge und Peakabstände in AtomVicinity-Dichteprofilen und der daraus folgenden Platzbesetzung sind die Simulationen wichtig, da sie zeigen, dass ein direkter Rückschluss aus den Feldverdampfungsfeldstärken für nicht geordnete Richtungen auf die Verhältnisse in einer geordneten Richtung nicht immer möglich ist. Letztendlich ermöglichen die Simulationen sogar, gänzlich auf Messungen in nichtgeordnete Richtungen zu verzichten.

Die von der kristallographischen Richtung abhängige Feldverdampfungsfeldstärke, die in dieser Arbeit erstmals quantitativ bestimmt werden konnte (Kap. 6.4.4.1), kann, wie anhand von W gezeigt wurde, ebenfalls durch die Simulation bestätigt werden. Die geometrische Anordnung der Nachbarn ist also entscheidend für die Feldverdampfungsfeldstärke.

Da die verwendete Simulation beliebige dreidimensionale Verteilungen von Atomen als Ausgangsdaten verwenden kann, lässt sich eine Vielzahl materialphysikalisch interessanter Situationen betrachten. Mit dem entwickelten Modell ist es somit nicht 
nur möglich, intermetallische Phasen, sondern beliebige Atomsondendaten zu simulieren. 


\section{Kapitel 8}

\section{Zusammenfassung}

Fokus der vorliegenden Arbeit war die Entwicklung einer Methode zur Platzbestimmung von ternären Elementen in intermetallischen Phasen. Zur korrekten Interpretation sind Feldverdampfungssimulationen hilfreich und teilweise notwendig. Der Vergleich von Experiment und Simulation erlaubt dabei die Bestimmung von Simulationsparametern, wobei vor allem die Möglichkeit der Bestimmung der A-BBindungsenergie von Interesse ist.

- XRD, TEM, FIM und TAP offenbaren eine $\mathrm{L} 1_{0} / \mathrm{D0}_{19}$-Lamellenstruktur für TiAl mit Zusätzen von Nb oder Cr. Wird Ag hinzulegiert, so liegt eine $\mathrm{L1}_{0}$-Matrix vor, in der sich nach Auslagerung Ag-reiche $\mathrm{L1}_{2}$-Ausscheidungen bilden.

- In L1 $1_{0}-\mathrm{TiAl}+\mathrm{X}$ konnten Relationen für die Feldverdampfungsfeldstärken $E_{\text {Evap }}$ der einzelnen Elemente für die nichtgeordnete [100]-Richtung festgestellt werden: $E_{E v a p, T i}<E_{E v a p, A l}$ (gilt immer), $E_{E v a p, A l}<E_{E v a p, N b}, E_{E v a p, T i}<E_{E v a p, A g}<$ $E_{\text {Evap }, A l}, E_{\text {Evap }, T i}<E_{\text {Evap }, C r}<E_{\text {Evap }, A l}$ für Al-arme Körner, $E_{\text {Evap }, C r} \leq$ $E_{E v a p, T i}<E_{E v a p, A l}$ für Al-reiche Körner

- Die experimentell bestimmten Relationen der Feldverdampfungsfeldstärken $E_{\text {Evap }}$ ließ sich durch Simulationen bestätigen. Die unterschiedlichen $E_{E v a p, C r}$ für verschiedene Körner sind durch unterschiedliche Platzbesetzung erklärbar, wobei in Al-armen Körnern vermehrt Al-Plätze belegt werden müssen.

- Die Platzbesetzung der Zusätze Ag,Cr und Nb in TiAl konnte experimentell ermittelt werden. $\mathrm{Nb}$ präferiert eindeutig Ti-Plätze. Lediglich in $\mathrm{Ti}_{42} \mathrm{Al}_{48} \mathrm{Nb}_{10}$ werden teilweise auch Al-Platzbesetzungen beobachtet. Ag belegt beide Plätze. 
Cr belegt ebenfalls beide Plätze. Zudem konnte für Cr nachgewiesen werden, dass die Platzbesetzung von der Zusammensetzung des Korns abhängt. Dabei ist nicht nur das Ti/Al-Verhältnis wichtig, sondern auch die Menge der hinzulegierten Cr-Atome.

- Durch Vergleich der AtomVicinity-Dichteprofile für zwei Überstrukturrichtungen in Experiment und Simulation von $\mathrm{Cu}_{3} \mathrm{Au}$ ließ sich die $\mathrm{Cu}-\mathrm{Au}$ Bindungsenergie $\Lambda_{1 . N N, C u-A u}=0.288 \mathrm{eV}$ angeben. Diese Bindungsenergie liefert zudem den quantitativ richtigen $\mathrm{Cu}-\mathrm{Au}-\mathrm{Peakabstand}$ der nichtgeordneten $<111>$ Richtung.

- Durch den Vergleich der, mittels DFT errechneten, Gesamtenergien von Einheitsund Superzellen ließen sich Bindungsenergieverhältnisse zweier Partner errechnen. Diese erklären die Platzbesetzung von Nb auf Ti-Plätzen im L1 $1_{0}$-Gitter, sowie die stark von den Konzentrationsverhältnissen abhängende Platzbesetzung von $\mathrm{Cr}$ und $\mathrm{Ag}$.

- Durch DFT berechnete Bindungsenergien ermöglichen Feldverdampfungssimulationen, die die Interpretation experimenteller Atomsondendaten, besonders im Hinblick auf die Platzbesetzung, unterstützen.

- Für Wolfram konnten von der kristallographischen Richtung abhängige Feldverdampfungsfeldstärken festgestellt und erstmals quantitativ ermittelt werden. Der Unterschied zwischen der $<110>$ und der $<121>$-Richtung beträgt hierbei $(3.3 \pm 1.6) \%$.

- Durch Vergleich der Abweichung der simulierten Spitzenkrümmung von Wolfram für verschiedene kristallographische Orientierungen mit experimentellen Ergebnissen konnte der, für die Berücksichtigung des externen Feldes in der Simulation wichtige, Straffaktor $D=0.44 \mathrm{~V} / \mathrm{nm}$ bestimmt werden.

Mit dieser Arbeit wurden Grundlagen gelegt, die es ermöglichen, anhand von Atomsondendaten die Platzbesetzung in intermetallischen Phasen beliebiger Elemente zu bestimmen. Dabei können sogar Isotope unterschieden werden, sowie leichte Elemente, die sich einer Analyse durch ALCHEMI entziehen, untersucht werden. Sogar eine sich verändernde Konzentration innerhalb eines Korns und die sich damit ändernde Platzbesetzung kann ermittelt werden. Es genügt, unter günstigen Bedingungen, eine zweistellige Anzahl von Atomen des hinzulegierten Typs in der Messung um die Bevorzugung einer Platzbesetzung zu ermitteln. Dies entspricht einigen $\mathrm{nm}^{3}$. 
Alleine anhand gut bekannter Materialparameter wie Sublimationsenergie, Ionisationsenergie und Austrittsarbeit konnten Feldverdampfungssimulation durchgeführt werden, durch die erstmals mittels experimenteller Atomsondendaten die AB-Bindungsenergie einer intermetallischen Phase bestimmt werden konnte. Somit wurde ein vergleichsweise einfacher Zugang zu einer Größe erlangt, die auch in der theoretischen Beschreibung anderer Effekte von Bedeutung ist. Unabhängig davon können alternativ durch DFT berechnete A-B-Bindungsenergien für die Simulation von Atomsondendaten beliebiger Materialien verwendet werden. 


\section{Literaturverzeichnis}

[AK1992] AL-KASSAB, Talaat: Frühstadien der Entmischung in binären Legierungen, untersucht mit der analytischen Feldionen-Mikroskopie, Uni Göttingen, Diss., 1992

[AK2007] Al-Kassab, Talaat; Yuan, Yong ; Kluthe, Christian ; Boll, Torben ; LIU, Zhi-Guo: Investigation of the ordering and atomic site occupancies of Nb-doped $\mathrm{TiAl} / \mathrm{Ti}_{3} \mathrm{Al}$ intermetallics. In: Surface and Interface Analysis 39 (2007), S. 257-261

[Bas1995] Bas, P. ; Bostel, A. ; Deconihout, B. ; Blavette, D.: A general protocol for the reconstruction of $3 \mathrm{D}$ atom probe data. In: Applied Surface Science 87/88 (1995), S. 298

[Bisw1982] Biswas, R. K. ; Forbes, R. G.: Theoretical arguments against the Müller-Schottky mechanism of field evaporation. In: J. Phys. D: Appl. Phys. 15 (1982), S. 1323

[Blah2001] Blaha, P. ; Schwarz, K. ; Madsen, G. K. H. ; Kvasnicka, D. ; Luitz, J.: WIEN2k, An Augmented Plane Wave + Local Orbitals Program for Calculating Crystal Properties. Karlheinz Schwarz, Techn. Universität Wien, Austria, 2001

[Boll2005] BoLL, Torben: Untersuchung der Platzbesetzung in $\gamma$-TiAlNb mittels Atomsondentomographie und Simulation. Universität Göttingen, 2005

[Boll2007] Boll, T. ; Al-Kassab, T. ; YuAn, Y. ; Liu, Z. G.: Investigation of the site occupation of atoms in pure and doped $\mathrm{TiAl} / \mathrm{Ti} 3 \mathrm{Al}$ intermetallic. In: Ultramicroscopy 107 (2007), Sep, Nr. 9, 796801. http://dx.doi.org/10.1016/j.ultramic.2007.02.011.-DOI 10.1016/j.ultramic.2007.02.011

[Bunt2007] Bunton, J. H. ; Olson, J. D. ; Lenz, D. R. ; Kelly, T. F.: Advances in Pulsed-Laser Atom Probe: Instrument and Specimen Design for Optimum Performance. In: Microscopy and Microanalysis 13 (2007), S. $418-427$

[Cald2002] Calderon, H. A. ; Garibay-Febles, V. ; Umemoto, M. ; YamaGUCHI, M.: Mechanical properties of nanocrystalline Ti-Al-X alloys. In: Materials Science and Engineering A 329-331 (2002), 196 - 205. 
http://dx.doi.org/DOI:10.1016/S0921-5093(01)01568-4. - DOI DOI: 10.1016/S0921-5093(01)01568-4. - ISSN 0921-5093

[Chen2003] Chen, J. ; Ishio, S. ; Sugawara, S.: Preparation of $\mathrm{L}_{0}$ ordered $\mathrm{CuAu}$ buffer layer and its effect on the $\mathrm{L}_{0}$ ordering in the FePt thin film. In: Thin Solid Films 426 (2003), Nr. 1-2, 211 - 215. http: //dx.doi.org/DOI:10.1016/S0040-6090(03)00032-4. - DOI DOI: 10.1016/S0040-6090(03)00032-4. - ISSN 0040-6090

[Corm2004] Cormen, T. H.: Algorithmen- Eine Einführung. Oldenbourg, 2004

[Deco2008] Deconihout, B. ; Vella, A. ; Vurpillot, F. ; Costa, G. D. ; Bostel, A.: 3D atom probe assisted by femtosecond laser pulses. In: Applied Physics A: Materials Science \& Processing 93 (2008), Nr. 4, S. 995-1003

[Dyke1953] Dyke, W. P. ; Trolan, J. K. ; Dolan, W. W. ; Barnes, George: The Field Emitter: Fabrication, Electron Microscopy, and Electric Field Calculations. In: Journal of Applied Physics 24 (1953), Nr. 5, S. 570

[Fark1994] FARKAS, D.: Interatomic potentials for Ti-Al without angular forces. In: Modelling Simul. Mater. Sci. Eng. 2 (1994), S. 975

[Fark1996] FARKAS, D. ; JonES, C.: Interatomic potentials for ternary Nb-Ti-Al alloys. In: Modelling Simul. Mater. Sci. Eng. 4 (1996), S. 23

[Forb1982] ForBEs, R. G.: An evaporation field formula including the repulsive ion-surface interaction. In: J. Phys. D.: Appl. Phys 15 (1982), S. L75

[Geis2007] Geiser, Brian P. ; Kelly, Thomas F. ; Larson, David J. ; Schneir, Jason ; RoBerTs, Jay P.: Spatial distribution maps for atom probe tomography. In: Microsc Microanal 13 (2007), Dec, Nr. 6, 437447. http://dx.doi.org/10.1017/S1431927607070948. - DOI 10.1017/S1431927607070948

[Gilc2001] Gilchrist, A. ; Pollock, T. M.: Cast gamma titanium aluminides for low pressure turbine blades: A design case study for intermetallics. In: Structural Intermetallics (2001)

[Gott2001] Gottstein, G.: Physikalische Grundlagen der Materialkunde. Springer-Verlag, 2001

[Haas1994] HaAsen, P.: Physikalische Metallkunde. Springer-Verlag, 1994

[Hao1999] HaO, Y.L. ; Xu, D.S. ; CUI, Y.Y. ; YAng, R. ; D. LI, B.: The site occupation of alloying elements in $\mathrm{TiAl}$ and $\mathrm{Ti}_{3} \mathrm{Al}$ alloys. In: Acta mater. 47 (1999), Nr. 4, S. 1129-1139

[Hao2000] HaO, Y. L. ; YAnG, R. ; CuI, Y. Y. ; LI, D.: The effect of Ti/Al ratio on the site occupancies of alloying elements in $\gamma$-TiAl. In: Intermetallics 8 (2000), Nr. 5-6, 633 - 636. http://dx.doi.org/DOI:10.1016/ 
S0966-9795(99)00138-7. - DOI DOI: 10.1016/S0966-9795(99)001387. - ISSN 0966-9795

[Hayd1980] Haydock, R. ; Kingham, D. R.: Post-Ionization of Field-Evaporated Ions. In: Phys. Rev. Lett. 44 (1980), Nr. 23, S. 1520

[Hohe1964] Hohenberg, P. ; Kohn, W.: Inhomogeneous Electron Gas. In: Phys. Rev. 136 (1964), Nov, Nr. 3B, S. B864-B871. http://dx.doi.org/ 10.1103/PhysRev.136.B864. - DOI 10.1103/PhysRev.136.B864

[Huan1991] Huang, Shyh-Chin ; Hall, Ernest L.: The effects of Cr additions to binary TiAl-base alloys. In: Metallurgical and Materials Transactions A 2 (1991), S. 2619-2627

[John1989] Johnson, R. A.: Alloy models with the embedded-atom method. In: Phys. Rev. B 39 (1989), Nr. 17, S. 12554

[Jone2003] JonEs, I.P.: Determining the locations of chemical species in ordered compounds: ALCHEMI. Version: 2003. http://dx.doi.org/DOI: 10.1016/S1076-5670(02)80015-5. Elsevier, 2003. - DOI DOI: 10.1016/S1076-5670(02)80015-5. - ISSN 1076-5670, 63 - 117, I

[Kim1997] Kim, S. ; Smith, G.D.W.: AP-FIM investigation on $\gamma$-based titanium aluminides. In: Materials Science and Engineering A 239-240 (1997), S. 229

[Kitt1969] KitTel, C.: Einführung in die Festkörperphysik. Oldebourg, 1969

[Kohn1965] Kohn, W. ; Sham, L. J.: Self-Consistent Equations Including Exchange and Correlation Effects. In: Phys. Rev. 140 (1965), Nov, Nr. 4A, S. A1133-A1138. http://dx.doi.org/10.1103/PhysRev.140.A1133. - DOI 10.1103/PhysRev.140.A1133

[Koni1986] Konitzer, D.G. ; Jones, I.P. ; Fraser, H.L.: Site occupancy in solid solutions of $\mathrm{Nb}$ in the intermetallic compounds $\mathrm{TiAl}$ and Ti3Al. In: Scripta Metallurgica 20 (1986), Nr. 2, 265 - 268. http: //dx.doi.org/DOI:10.1016/0036-9748(86)90139-0. - DOI DOI: 10.1016/0036-9748(86)90139-0. - ISSN 0036-9748

[Kres2009] KRESSE, Thomas: Untersuchung der Platzbesetzung von Titan in $\mathrm{D0}_{3^{-}}$ geordneten Fe-26at.\% Al, Universität Göttingen, Diplomarbeit, 2009

[Kreu1992] Kreuzer, H. J. ; WANG, L. C.: Self-consistent calculation of atomic adsorption on metals in high electric fields. In: Phys. Rev. B 45 (1992), Nr. 20, S. 12050

[Liu1991] LiU, J. ; WU, C. ; Tsong, T. T.: Measurement of the binding energy of kink-site atoms of metals and alloys. In: Phys. Rev. B 43 (1991), Nr. 14, S. 11595

[Mass1990] Massalski, Thaddeus B. ; Massalski, Thaddeus B. (Hrsg.): Binary alloy phase diagrams. ASM International, 1990 
[Meno1996] Menon, E. S. K. ; Fox, A. G. ; Mahapatra, R.: Accurate determination of the lattice parameters of $\gamma$-TiAl alloys. In: Journal of Materials Science Letters 15 (1996), Nr. 14, S. 1231-1233

[Mill1996] Miller, M. K. ; Cerezo, A. ; Hetherington, M. G. ; Smith, G.D.W.: Atom Probe Field Ion Microscopy. Oxford Science Publications, 1996

[Moha1991] Mohandas, E. ; Beaven, P.A.: Site occupation ofNb, V, Mn and Cr in $\gamma$-TiAl. In: Scripata Met. Mat. 25 (1991), S. 2023-2027

[Mood2009] Moody, Michael P. ; Gault, Baptiste ; Stephenson, Leigh T. ; Haley, Daniel ; Ringer, Simon P.: Qualification of the tomographic reconstruction in atom probe by advanced spatial distribution map techniques. In: Ultramicroscopy 109 (2009), Jun, Nr. 7, 815824. http://dx.doi.org/10.1016/j.ultramic.2009.03.016. - DOI 10.1016/j.ultramic.2009.03.016

[Muel1969] Mueller, E. W. ; Tsong, T. T.: Field Ion Microscopy: Principles and Applications. Elsevier, 1969

[NI2001] NI, Xiaodong ; CHEN, Guoliang ; WANG, Xitao ; HUI, Xudong: MEAM Simulation of Nb Atoms in TiAL+Nb System. In: J. Mater. Sci. Technol. 18 (2001), S. 1-5

[Pasi1992] Pasianot, R. ; Savino, E. J.: Embedded-atom-method interatomic potentials for hcp metals. In: Phys. Rev. B 45 (1992), Nr. 22, S. 12704

[Perd1996] Perdew, John P. ; Burke, Kieron ; WANG, Yue: Generalized gradient approximation for the exchange-correlation hole of a manyelectron system. In: Phys. Rev. B 54 (1996), Dec, Nr. 23, S. 1653316539. http://dx.doi.org/10.1103/PhysRevB.54.16533. - DOI 10.1103/PhysRevB.54.16533

[Petz1993] Petzow ; Petzow, G. (Hrsg.) ; Effenberg, G. (Hrsg.): Ternary Alloys. Bd. 7. VCH Verlagsgesellschaft, 1993

[Revi1993] Reviere, R.D. ; Chen, X.F. ; Oliver, B.F. ; Brooks, C.R. ; DunLAP, J.R.: Substitution behavior of $\mathrm{Mn}, \mathrm{Cr}$, and $\mathrm{Zr}$ in ternary and quaternary alloys of TiAl. In: Materials Science and Engineering: A 172 (1993), Nr. 1-2, 95 - 100. http://dx.doi.org/DOI:10.1016/ 0921-5093 (93) 90429-I. - DOI DOI: 10.1016/0921-5093(93)90429-I. - ISSN 0921-5093

[Ross1996] Rossouw, C. J. ; Forwood, C. T. ; Gibson, M. A. ; Miller, P. R.: Zone-axis convergent-beam electron diffraction and ALCHEMI analysis of Ti-Al alloys with ternary additions. In: Philosophical Magazine, Part A 74 (1996), Juli, S. 77-102. http://dx.doi.org/10.1080/ 01418619608239691. - DOI 10.1080/01418619608239691 
[Star2007] Starostenkov, M. D. ; Dudnik, E. A.: Investigation of changes in the order parameters near antiphase boundaries in the $\mathrm{Cu}_{3} \mathrm{Au}$ alloy. In: Bulletin of the Russian Academy of Sciences: Physics 71 (2007), S. 1934-9432

[Terb2003] Terblans, J. J.: Calculated bulk vacancy formation energy $E_{v}$ for a Schottky defect in $\mathrm{Al}$ single crystals. In: Surface and Interface Analysis 35 (2003), Nr. 6, S. 548-551

[Toul2002] Toulemonde, M. ; Assmann, W. ; Trautmann, C. ; Grüner, F.: Jetlike Component in Sputtering of LiF Induced by Swift Heavy Ions. In: Phys. Rev. Lett. 88 (2002), Jan, Nr. 5, S. 057602. http://dx. doi.org/10.1103/PhysRevLett.88.057602. - DOI 10.1103/PhysRevLett.88.057602

[Vurp1999] Vurpillot, F. ; Bostel, A.; Menand, A. ; Blavette, D.: Trajectories of Field emitted ions in 3D atom-probe. In: Eur. Phys. J. AP 6 (1999), S. 217

[Vurp2000] Vurpillot, F. ; Bostel, A. ; Blavette, D.: Trajectory overlaps and local magnification in three dimensional atom probe. In: Appl. Phys. Lett. 76 (2000), Nr. 21, S. 3127

[Vurp2001a] Vurpillot, F. ; Bostel, A. ; Blavette, D.: A new approach to the interpretation of atom probe field-ion microscopy images. In: Ultramicroscopy 89 (2001), Oct, Nr. 1-3, S. 137-144

[Vurp2001b] Vurpillot, F. ; Costa, G. da ; Menand, A. ; Blavette, D.: Structural analyses in three-dimensional atom probe: a Fourier transform approach. In: Journal of Microscopy 203 (2001), S. 295

[Vurp2004a] Vurpillot, F. ; Cerezo, A. ; Blavette, D. ; Larson, D. J.: Modeling image distortions in 3DAP. In: Microsc Microanal 10 (2004), Jun, Nr. 3, 384-390. http://dx.doi .org/10.1017/S1431927604040486. DOI $10.1017 /$ S1431927604040486

[Vurp2004b] Vurpillot, F. ; LARson, D. ; Cerezco, A.: Improvement of multilayer analyses with a three-dimensional atom probe. In: Surf. Interface Anal. 36 (2004), S. 552

[Vurp2007] Vurpillot, F. ; Gilbert, M. ; Vella, A. ; Deconihout, B.: Femtosecond Laser Atom Probe Tomography: Principles and applications. In: Microsc Microanal 13 (2007)

[Wese1995] Wesemann, J. ; Frommeyer, G. ; Kreuss, M.: APFIM investigations on ordered [gamma]-TiAl using single-layer detection method. In: Applied Surface Science 87-88 (1995), 179 - 184. http: //dx.doi.org/DOI:10.1016/0169-4332(94)00483-8. - DOI DOI: 10.1016/0169-4332(94)00483-8. - ISSN 0169-4332. - Proceedings of the 41st International Field Emission Symposium 
[Will2009] WiLle, Catharina G.: Investigation of the segragation behaviour in nanocrystalline materials, Universität Göttingen, Diss., 2009

[Wolf1996] Wolf, W. ; Podloucky, R. ; Rogl, P. ; Erschbaumer, H.: Atomic modelling of Nb, V, Cr and Mn substitutions in [gamma]-TiAl. 2: Electronic structure and site preference. In: Intermetallics 4 (1996), Nr. 3, 201 - 209. http://dx.doi.org/DOI:10.1016/0966-9795(95) 00036-4. - DOI DOI: 10.1016/0966-9795(95)00036-4. - ISSN 09669795

[Wood1998] Woodward, C. ; Kajihara, S. ; Yang, L. H.: Site preferences and formation of substitutional $\mathrm{Si}, \mathrm{Nb}, \mathrm{Mo}$, Ta, and $\mathrm{W}$ solid solutions in L1 $1_{0}$ TiAl. In: Phys. Rev. B 57 (1998), Nr. 21, S. 13459

[Yang2000] Yang, R. ; HaO, Y. ; Song, Y. ; Guo, Z. X.: Site occupancy of alloying additions in titanium aluminides and its application to phase equilibrium. In: Z. Metallkd. 9 (2000), S. 296

[Zamb2009] Zambaldi, Claudio ; Zaefferer, Stefan ; Wright, Stuart I.: Characterization of order domains in $\gamma$-TiAl by orientation microscopy based on electron backscatter diffraction. In: Journal of Applied Crystallography 42 (2009), Dec, Nr. 6, 1092-1101. http://dx.doi.org/ 10.1107/S0021889809036498. - DOI 10.1107/S0021889809036498

[Zope2003] Zope, R. R. ; Mishin, Y.: Interatomic potentials for atomistic simulations of the Ti-Al system. In: Phys. Rev. B 68 (2003), S. 24102

[Zurl2003] Zurlev, D. N. ; Forbes, R. G.: Field ion emission: the effect of electrostatic field energy on the prediction of evaporation field and charge state, Rapid Communication. In: J. Phys. D: Appl. Phys 36 (2003), S. L74 


\section{Kapitel A}

\section{Quellcodeauszüge SIMTAP}

Die Quellcodes sind in einer sehr kleinen Schriftgröße damit überhaupt eine übersichtliche Darstellung möglich ist. Insbesondere die Simulation benötigt eine große Zahl an Parametern. Es sind hier lediglich Auszüge der Codes vorgestellt, um das Prinzip zu erläutern. Komplette Codes sind auf Anfrage beim Autor erhältlich.

Die Datenstruktur der Atome ist folgendermaßen definiert:

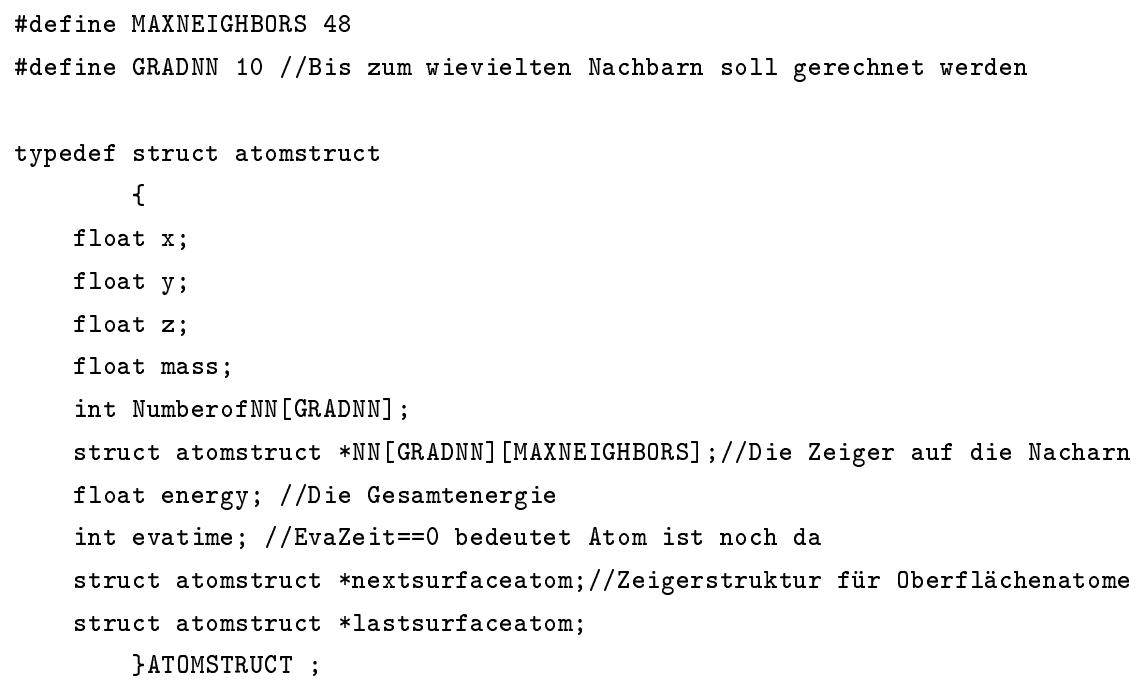

Die zur Simulation benötigten Parameter werden überwiegend in einem Konfigurationsfile übergeben. Die zugrunde liegende Kristallstrunktur, der Zylinderradius der Spitze sowie der Spitzenradius werden in der Benutzeroberfläche des Programms eingegeben. Dies ist das übliche Konfigurationsfile für die TiAlNb-Simulationen ( "\#" kennzeichnet eine Kommentarzeile) :

\#Config-File fuer SimTap

\#Es gibt 23 Parameter in der Version 1.52 des Configfiles 
\#Versionsnummer

1.52

\#Anzahl der Nächsten Nachbarn (Maximal 12)

12

\#Masse des 1. Elements

27

\#Masse des 2. Elements

48

\#Masse des 3. Elements

93

\#Nächste-Nachbarn-Bindungsenergie des 1.Elements mit dem 1.Element $1-1$ (in eV)

0.213

\#Bindungsenergie NN 2-2

0.56

\#Bindungsenergie NN 3-3

0.519

\#Bindungsenergie NN 1-2

0.493

\#Bindungsenergie NN 1-3

0.503

\#Bindungsenergie NN 2-3

0.652

\#NN-Abstand 1-1 (kleinster Abstand der Atome in der Paarung) (in Angstroem)

2.86

\#NN-Abstand 2-2

2.86

\#NN-Abstand 3-3

2.86

\#NN-Abstand 1-2

2.84

\#NN-Abstand 1-3

2.84

\#NN-Abstand 2-3

2.84

\#Ionisationsenergie (Ionsiationsenergie 1. Grad +2. Grad) -2*Austrittsarbeit für 1 16.6

\#Ionisationsenergie (Ionsiationsenergie 1. Grad +2. Grad) -2*Austrittsarbeit für 2 
12.4

\#Ionisationsenergie (Ionsiationsenergie 1. Grad +2. Grad) -2*Austrittsarbeit für 3 13.2

\#Straffaktor für Abweichung von der Idealen Oberfläche in eV pro Angstroem 0.44

\# Art der Ausgabe $0=o h n e$ Veraenderung

\# Andere Parameter verändern die z-Komponente der Atome

\# 1=Anzahl der NN 2=Anzahl der 2.NN

\# 3=Strafenergie 4=Bindungsenergie+Ionisationsenergie+Austrittsarbeit(Un)

\# $5=$ Abstand zum letzten Atom in z-Richtung

\# 6=Abstand zum letzten Atom in $x, y$-Richtung

\# 7=x-Position des letzten Atoms

0 


\section{Kapitel B}

\section{Al8at.\%Li: Ein nicht ganz so einfaches System}

Um die Möglichkeit der Abschätzung von Feldverdampfungsfeldstärken zu demonstrieren wurde neben Ni8at.\% Al auch als weiteres binäres System Al8at.\%Li untersucht. Allerdings ist dieses System nicht einfach, sondern befindet sich bereits in einem Frühstadium der Entmischung [AK1992].

Die nominellen Konzentrationen stimmen mit den im TAP gemessenen Al4.0at.\%Li nicht gut überein. Dies erklärt sich, wenn man berücksichtigt, dass die Konzentration in verschiedenen Körnern abweichen kann. Nach dem Phasendiagramm kann man erwarten, dass die Al-Phase vorliegt. Die Auswahl für AtomVicinity befand sich um einen (mutmaßlich- das FIM-Bild ließ eine eindeutige Indizierung nicht zu) (002)-Pol. Zur Bestimmung der Dichteprofile wurden die Eben senkrecht zur $z$-Achse ausgerichtet. Die sich ergebenden AtomVicinityDichteprofile sind in Abb. $6.24 \mathrm{zu}$ sehen. Die Ebenabstände wurden durch Anpassung des Parameter E $\beta$ auf die erwarteten Werte von (002)-kfz-Aluminium (0.202 nm) eingestellt.

Es lässt sich aus den Zentralpeaks eine Abschätzung von $E_{E v a p, A l}>E_{E v a p, L i}$ angeben, die gut zu dem erwarteten extrem niedrigen $E_{\text {Evap }, L i}=14 \mathrm{~V} / \mathrm{nm}$ im Vergleich zu minimal erwarteten $19 \mathrm{~V} / \mathrm{nm}$ für Al passt. Auffällig ist in diesem Fall jedoch, dass in der Li-Kurve auf den ersten Blick zu wenige Peaks vorhanden sind. Wenn man die Al-Kurve betrachtet findet man allerdings auch dort eine geringere Dichte für die um den Zentralpeak gruppierten Peaks. Setzt man dies gedanklich für einen Li-Peak mit schwachem Abfall nach rechts fort, so ist erklärbar warum 2 Peaks als ein Peak auftauchen. Der Grund für die geringeren Peakdichten kann allerdings auch aus einer 


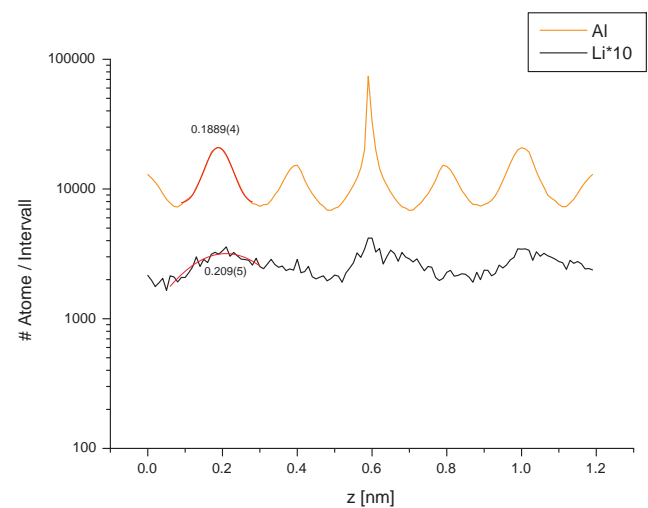

(a) Al8at.\%Li: Referenzatom $=\mathrm{Al}$

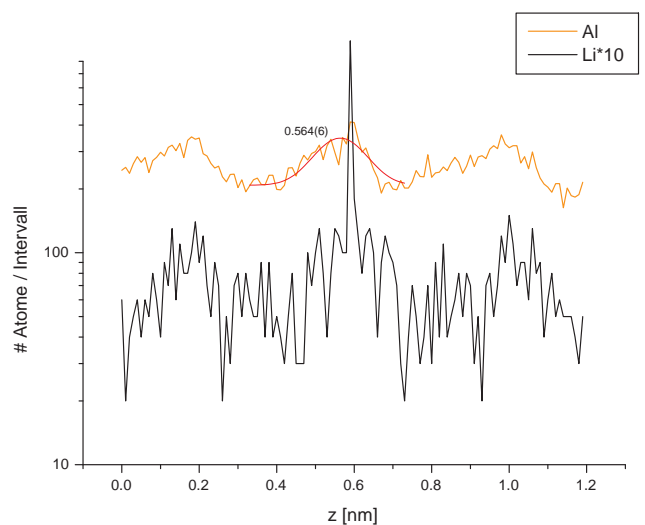

(b) Al8at.\%Li: Referenzatom $=\mathrm{Li}$

Abbildung B.1: AtomVicinity-Dichteprofile von Al8at.\%Li. Man sieht, dass sich, unabhängig davon, welches Element als Referenzatom definiert wird, eine Peakreihenfolge erkennbar ist. Die an den Peaks notierten Werte sind die z-Positionen aus dem Gaussfit. In (a) ist auffällig, dass die Al-Peaks nahe dem Zentralpeak zu klein sind. Deshalb verschwimmen jeweils zwei Li-Peaks mit Flanken nach rechts zu Einem. Die Reihenfolge lässt sich dennoch (notfalls am Zentralpeak) als Al-Li ausmachen. In (b) kann aus dem Zentralpeak die Reihenfolge Al-Li abgelesen werden. Aufgrund des, dank des lokalen Vergrößerungseffekts, geringen Li-Anteils (2at.\%) im Pol ist die Statistik schlecht. Es tauchen unerwartet zusätzliche Li-Peaks im halben Ebenenabstand auf.

beginnenden Nahordnung einer Li-reichen intermetallischen Phase herrühren. Die Ausbildung einer Nahordnung für eine Al-reiche Phase kann es nicht dafür verwantwortlich sein, da dann in B.1 die Li-Peaks im einfachen Abstand verschwinden und dafür Al-Peaks auftauchen müssten. Die These einer Keimbildung wird durch ein in z-Achse gelegtes Konzentrationsprofil gestützt, in dem Konzentrationsunterschiede von 2 at. $\%$ fesstellbar sind. 


\section{Danksagung}

Wie immer zuletzt kommt an dieser Stelle die Danksagung. In unserer Gesellschaft wird häufig gedankt. Meist ist es eher eine formale Äußerung, die den Respekt zum Gegenüber verdeutlichen soll. Da die Danksagung Teil jeder mir bekannten Dissertation ist könnte man dies auch für solche Danksagungen so sehen.

So ist es allerdings nicht, weder hier noch in anderen Arbeiten. Ich möchte hier bekennen, dass die vorliegende Arbeit ohne die Hilfe der erwähnten und auch einiger nicht erwähnter Personen so nie hätte erstellt werden können.

Besonderer Dank gebührt Prof. Talaat Al-Kassab und Prof. Reiner Kirchheim für die langjährige Betreuung und zahlreiche fachliche Gespräche. Auch dem Koreferenten Prof. Markus Münzenberg sei gedankt. (Und natürlich den anderen Professoren des Prüfungsgremiums.)

Großer Dank gebührt natürlich der FIM-Gruppe, besonders meinen "Zimmermitnutzern" Dr. Carsten Nowak und Tobias Schulz sowie Thomas \& Thomas, die beide ebenfalls an intermetallischen Phasen gearbeitet haben und deren Probleme mir gezeigt haben, dass mein Material, relativ gesehen, doch recht gutmütig ist. Außerdem war Michael Sobol eine große Hilfe bei den sich stellenden Programmieraufgaben.

Ebenfalls gedankt werden muss unseren chinesischen Kooperationspartnern Prof. Zhi-Guo Liu, Dr. Yong Yuan sowie Dr. Lei Shi. Die untersuchten TiAl-Proben wurden allesamt an der Nanjing Universität in China hergestellt und die TEMAufnahmen zum großen Teil von Dr. Yong Yuan gemacht.

Auch Dr. Zhiyong Zhu von der King Abdullah University in Saudi-Arabien muss an dieser Stelle gewürdigt werden. Ihm sind die DFT-Berechnungen zu verdanken.

Nicht zuletzt möchte ich auch meiner Familie, insbesondere meinen Eltern und meinem Bruder Björn, für die jahrelange moralische Unterstützung danken. 
142 KAPITEL B. AL8AT.\%LI: EIN NICHT GANZ SO EINFACHES SYSTEM 


\section{Lebenslauf}

\section{Angaben zur Person}

$\begin{array}{ll}\text { Name } & \text { Torben Boll } \\ \text { Geburtsdatum und -ort } & \text { 02.10.1979, Göttingen } \\ \text { Familienstand } & \text { ledig } \\ \text { Staatsangehörigkeit } & \text { deutsch }\end{array}$

\section{Ausbildung}

06/1999

Allgemeine Hochschulreife, Max-Planck-Gymnasium (Göttingen)

11/1999 - 08/2000 Bundeswehr in Stadtoldendorf

10/2000- 01/2006 Studium der Physik an der Universität Göttingen

$10 / 2002$

Vordiplom im Fach Physik

$01 / 2006$

Diplom Physik an der Georg-August-Universität zu Göttingen mit dem Diplomarbeitstitel "Untersuchung der Platzbesetzung in $\gamma$-TiAlNb mittels Atomsondentomographie und Simulation"

seit $03 / 2006$ Wissenschaftlicher Mitarbeiter an der Georg-AugustUniversität zu Göttingen am Institut für Materialphysik in der Arbeitsgruppe von Prof. Dr. R. Kirchheim 